\title{
Diffusion Weighted Magnetic Resonance Imaging Assessment of Blood Flow in the Microvasculature of Abdominal Organs
}

\author{
by \\ Loredana Sorına Truica \\ M Sc (Unıversity of Bucharest) 2001
}

\begin{abstract}
A thesis submitted to the
Faculty of Graduate Studies and Postdoctoral Affairs

in partial fulfillment of the requirements

for the degree of

Doctor of Philosophy

(Physics)
\end{abstract}

Ottawa-Carleton Institute for Physics

Department of Physics

Carleton Unıversity

January, 2011

(C) 2011, Loredana Sorına Truica 
Library and Archives

Canada

Published Heritage Branch

395 Wellington Street Ottawa ON K1A ON4 Canada
Bibliothèque et

Archives Canada

Direction du

Patrımoıne de l'édition

395, rue Wellington

Ottawa ON K1A ON4

Canada
Your file Votre référence

ISBN 978-0-494-79622-1

Our file Notre référence

ISBN 978-0-494-79622-1
NOTICE

The author has granted a nonexclusive license allowing Library and Archives Canada to reproduce, publish, archive, preserve, conserve, communicate to the public by telecommunication or on the Internet, loan, distribute and sell theses worldwide, for commercial or noncommercial purposes, in microform, paper, electronic and/or any other formats

The author retains copyright ownership and moral rights in this thesis Neither the thesis nor substantial extracts from it may be printed or otherwise reproduced without the author's permission

\section{AVIS}

L'auteur a accordé une licence non exclusive permettant à la Bıblıothèque et Archives Canada de reproduire, publier, archiver, sauvegarder, conserver, transmettre au public par télécommunıcatıon ou par l'Internet, prêter, distribuer et vendre des theses partout dans le monde, à des fins commerciales ou autres, sur support microforme, papıer, électronıque et/ou autres formats

L'auteur conserve la propriété du droit d'auteur et des droits moraux qui protege cette these $\mathrm{N}_{\mathrm{I}}$ la thèse nı des extraıts substantıels de celle-cl ne doivent être ımprımés ou autrement reproduits sans son autorisation
In compliance with the Canadian Privacy Act some supportıng forms may have been removed from this thesis

While these forms may be included in the document page count, their removal does not represent any loss of content from the thesis
Conformément à la loı canadienne sur la protection de la vie privée, quelques formularres secondaires ont ete enlevés de cette thèse

Bien que ces formulaires aient inclus dans la pagination, il n'y aura aucun contenu manquant 


\section{Abstract}

In this thesis, water diffusion in human liver and placenta is studied using diffusion weighted magnetıc resonance ımaging For short, randomly onented vascular segments, intravascular water motion is diffusion-like For tissues with large vascular compartments the diffusion decay is bi-exponential with one component corresponding to diffusing water and the other to water in the microvasculature This model, known as the intravoxel incoherent motion (IVIM) model, is seldom used with abdominal organs because of motion artifacts This limitation was overcome for the experiments reported here by introducing 1) parallel ımaging, 2) navigator echo respiratory triggering (NRT), 3) a double echo diffusion sequence that inherently compensates for eddy current effects, 4) SPAIR fat suppression and 5) a superior approach to image analysis In partıcular, the use of NRT allowed us to use a free breathing protocol instead of the previously required breath hold protocol The resulting DWI images were of high quality and motion artifact free Diffusion decays were measured over a larger portion of the decay than had previously been reported and the results are considerably better than those previously reported

For both studies, reliable measurements of the diffusion coefficient $(D)$, pseudodiffusion coefficient $(D)$ and perfusion fraction $(f)$, were obtained using a region of 
interest analysis as well as a pixel-by-pixel approach To within experımental error, all

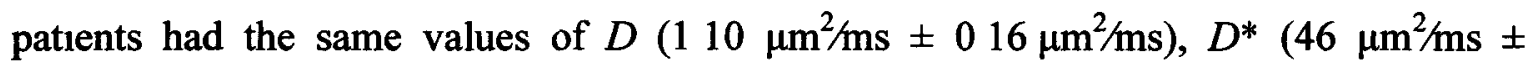
$\left.17 \mu \mathrm{m}^{2} / \mathrm{ms}\right)$ and $f(440 \% \pm 69 \%)$ in liver and $D\left(18 \mu \mathrm{m}^{2} / \mathrm{ms} \pm 02 \mu \mathrm{m}^{2} / \mathrm{ms}\right), D^{*}(30$ $\left.\mu \mathrm{m}^{2} / \mathrm{ms} \pm 12 \mathrm{~m}^{2} / \mathrm{ms}\right)$, and $f(40 \% \pm 6 \%)$ in the placenta No dependence on gestational age was found for the placental study Parametric maps of $f$ and $D^{*}$ were consistent with blood flow patterns in both systems The model worked well for both investigated organs even though their anatomical structures are quite different

A method for removing rectified noise bias from low intensity magnitude MR images measured with phased array coils is also presented This algorithm has significance for diffusion decay measurements since it permits the use of low intensity data points which could, for example, allow the acquisition of high resolution parametric maps 


\section{Acknowledgements}

I would like to express my sincere gratitude to my supervisor, Dr Ian Cameron for the guidance and constant support provided throughout the course of this work This thesis would not appear in its present form without his kind assistance and valuable suggestions I would like to thank my professors at Carleton University for sharing their knowledge and especially professor Paul Johns for great teachıng, guidance and support

I'm grateful to all members of the Diagnostıc Imaging - Magnetıc Resonance Lab I had the pleasure to know and work with Ian Cameron, Greg Cron, Claire Foottit, Elena Olariu, Fakhereh Mirrashed, Andra Smith and Ewan Hill They were not only colleagues, they were "a great family" as well

I would like to acknowledge Drs Andre Gruslın, Len Avruch and Felipe Morettı for their valuable input and assistance in the Placenta project In particular, I would like to thank the technologists for their help in the acquisition of the data and the gentle and attentive handling of the volunteers involved in the above mentioned project I would also like to thank all the volunteers from the liver study

My parents, mama Anitza and my sister have shown me unconditıonal love and support and whispered guidance throughout the years of my life I love, miss and thank 
them from my heart My gratıtude and warm thoughts go towards all my friends around this blue planet Especially Corına, Marius and Cristina, an invisible and constant presence, thank you for an additıonal "proof" that tıme and distance are relative

To my closest friends here, Miha1, Maria and Elena and my adopted "family" Miriam and Jonathan, great thanks for their love, support and wonderful memories together

Heartfelt thanks go to much missed, Ticu and Nenea Nicu for being "my other parents" and to Nic for showing me the way to dreams and life Their presence from somewhere between the stars has been always felt

Thank you all 


\section{Table of Contents}

Abstract

Acknowledgements $\quad$ iv

Table of Contents _ v1

$\begin{array}{ll}\text { List of Tables } & \text { X1 }\end{array}$

List of Figures $\quad$ X111

List of Abbreviations $\quad$ XV111

1. Introduction 1

11 Historical Introduction 2

12 Introduction to Diffusion in Magnetic Resonance 2

121 Intravoxel Incoherent Motion 6

13 Abdomınal Imagıng Literature Review $\quad 7$

131 IVIM Perfusion versus Classical Perfusion 9

14 Thesis Outline $\quad 10$ 
21 Introduction 14

22 Principles of Nuclear Magnetıc Resonance 14

221 The Nuclear Magnetic Moment in a Statıc Magnetıc Field 14

222 Nuclear Spin Energy Level Population in a Statıc Magnetıc

$\begin{array}{ll}\text { Freld } & 17\end{array}$

223 Effect of RF Pulses and Magnetic Field Gradients on $\boldsymbol{M}$

224 The Bloch Equations and Relaxation 20

23 Diffusion 25

231 Diffusion Theory 25

2311 Fick's law and Einstein's equation 25

2312 Diffusion and NMR 27

2313 Eddy Currents 32

2314 Equations governing the diffusive transport of magnetization $\quad 34$

232 Apparent Diffusion in Biological Tissues 36

233 Intravoxel Incoherent Motion Imaging 38

2331 Intravoxel Motion and MR Imaging 38

24 Motion Tracking with Prospective Acquisition Correction (PACE) 45

25 Human Placenta 49

251 Placenta Development 50

252 Blood Circulation in the Placenta 52

26 Human Liver 53 
261 Structural Anatomy of the Liver

262 Functional Anatomy of the Liver $\quad 54$

3. Using an IVIM Model to Assess Liver Microvasculature 57

31 Introduction $\quad 58$

32 Materials and Methods $\quad 61$

321 Subject Population $\quad 61$

$322 \mathrm{MR}$ Technıque $\quad 62$

3221 Breath hold protocol 63

3222 Navigator echo respiratory triggered free breathıng protocol

323 Image Analysis $\quad 65$

$\begin{array}{lll}33 & \text { Results } & 69\end{array}$

331 Perfusion Fraction $\quad 72$

332 Diffusion Coefficient $\quad 76$

333 Pseudo-Diffusion Coefficient 79

34 Discussion $\quad 82$

341 Comparison of the IVIM Parameters for Different ROIs 85

342 Breath Hold Protocol versus Free Breathing Protocol 87

343 Comparison with IVIM Parameter Values in the Literature 92

3431 The IVIM study of Yamada et al 94

3432 The IVIM study of Lucianı et al 95

$\begin{array}{lll}35 & \text { Conclusions } & 100\end{array}$ 
4. Using an IVIM Model to Assess Blood Flow in the Placenta 102

41 Introduction 103

42 Materıals and Methods 104

421 Subject Population $\quad 104$

422 MR Technique 105

423 Image Analysis $\quad 107$

43 Results 110

431 IVIM Parametric Maps 111

432 IVIM Parameters for Different ROIs 111

433 IVIM Parameters Versus Gestational Age 119

434 IVIM Parameters for $\mathrm{ROI}_{\mathrm{n}}$ and $\mathrm{ROI}_{\text {out }}$

44 Discussion 126

441 Placental IVIM Studies in the Literature 131

442 Comparison of our IVIM Parameters with the Literature $\quad 134$

4421 Diffusion Coefficient 135

4422 Pseudo-Diffusion Coefficient 136

4423 Perfusion Fraction 136

443 WP-ROI versus U-ROI 137

$444 \mathrm{ROI}_{\text {nn }}$ versus $\mathrm{ROI}_{\text {out }} \quad 138$

445 Coefficients of Variation for the IVIM Parameters $\quad 140$

446 Discussion of IVIM Parameters 142

45 Conclusions 147 
5. Rectified Noise Bias Correction

51 Introduction 151

52 Theory of Rectıfied Noise Bias Correction 153

521 Background Theory 153

522 Extension of the Rectıfied Noise Bias Correction Algorithm 156

53 Methods 158

$\begin{array}{lll}54 & \text { Results } & 161\end{array}$

55 Discussion 165

56 Conclusions 168

$6 \quad$ General Conclusions............................................... 169

$\begin{array}{lll}\text { Appendix A Liver Statıstics } & 173\end{array}$

$\begin{array}{lll}\text { Appendix B Placenta Statistics } & 178\end{array}$

Appendix C $\widetilde{\theta}$ as a Function of $\theta$ and $N$ Calculated Using $\mu_{\chi}$ and $\sigma_{g} \quad 183$

Appendix D $\tilde{\theta}$ as a Function of $\theta$ and $N$ Calculated Using $\left\langle M_{N}\right\rangle_{\chi 9}$ and $\sigma_{g} \quad 185$

Appendix E Simulated PDFs Before and After the RNBC Calculated Using 187 $\left\langle M_{N}\right\rangle_{\chi 9}$ and $\sigma_{g}$ for Different $N$ Element Coils

References 


\section{List of Tables}

31 Mean $D, D^{*}$, and $f$ values for the right side of the liver for the FB-protocol 72

32 Mean $D, D^{*}$, and $f$ values for the right side of the liver for the BH-protocol 72

33 Mean $D, D^{*}$, and $f$ values for the right side of the liver for the FB-protocol when a $t$-test was performed, and a two talled $p$-value is reported

34 IVIM parameter values for the right side of the liver measured with our FBprotocol compared with those obtained by Lucianı et al [9] with a pneumatic belt RT sequence

35 IVIM parameter values for the right side of the liver measured with our BHprotocol compared with those obtained by Yamada et al [12] with their BH-protocol

36 Mean IVIM parameter values averaged over the right side of the liver (Ottawa) and the Luciann 19] data, when a $t$-test was performed, and a two tailed $p$-value is reported

37 IVIM parameter values for the right side of the liver measured with our FBprotocol compared with those obtained by Patel et al [95] using an NRT sequence

41 Slopes for the IVIM parameters vs gestational age

42 Mean IVIM parameter values averaged over $\mathrm{GA}=18$ to 35 weeks

43 Mean IVIM parameter values averaged over $\mathrm{GA}=18$ to 35 weeks (Ottawa) and the Notthingam data, when a $t$-test was performed, and a two talled $p$ value is reported

44 Mean IVIM parameter values averaged over all subjects

51 Computed estımates of $\theta_{N}$ as a function $A$ and $N$ for the case where the 
exact values of $\mu_{\chi}$ and $\sigma_{g}$ are used

Appendix A Liver statıstics

173

Appendix B Placenta Statıstıcs

178

Appendix $C \tilde{\theta}$ as a function of $\theta$ calculated using $\mu_{\chi}$ and $\sigma_{g}$ for $N=1,2,4,8,16 \quad 160$

Appendix D $\widetilde{\theta}$ as a function of $\theta$ calculated using $\left\langle M_{N}\right\rangle_{\chi 9}$ and $\sigma_{\mathrm{g}}$ for $N=1,2,4$, 8,16

162 


\section{List of Figures}

21 Precession of $\boldsymbol{\mu}$ about $\boldsymbol{B}_{0}$ at the Larmor angular frequency $\omega_{0}=\gamma B_{0}$ The angle $\theta$ represents the phase of the precession

22 Energy level diagram for a nuclear spin with $I=1 / 2$ in a static magnetic field, $\boldsymbol{B}_{0} \Delta E=\gamma \hbar B_{0}$ represents the energy difference between levels separated by $\Delta m= \pm 1$ The bold line schematically represents the relative population in each state for an ensemble of spins at thermal equilibrium

23 (a) The total magnetic field, $\boldsymbol{B}_{\text {total }}$, in the laboratory frame $(\mathrm{x}, \mathrm{y}, \mathrm{z})$

(b) The effective field, $\boldsymbol{B}_{\text {eff }}$, in the RRF ( $\left.x^{\prime}, y^{\prime}, z^{\prime}\right)$ which rotates at angular frequency $\omega$

24 (a) The trajectory of $M$ in the laboratory frame (x, y, z) in the presence of both 1) a longitudinal static magnetic field, $\boldsymbol{B}_{0}$, and 2) a transverse rotatıng field $\boldsymbol{B}_{1}$ On resonance $\left(\omega=\omega_{0}\right), \boldsymbol{M}$ precesses simultaneously about both $\boldsymbol{B}_{0}$, at frequency $\omega_{0}$, and $\boldsymbol{B}_{1}$, at frequency $\omega_{1}$, where $\omega_{0} \gg \omega_{1}$ (b) The trajectory of $\boldsymbol{M}$ in the RRF (x', y', z') on resonance For this case the longitudinal effective magnetic field will be zero

25 Diagrams of (a) longitudinal magnetization $M_{z}$ and (b) transverse 
magnetization $M_{x y}$ relaxation as a function of time, $t$

26 The Stejskal-Tanner Pulsed-Gradient Spın Echo (PGSE) sequence where $\delta$ is the pulse duration, $\Delta$ is the diffusion time, $G$ is the gradient amplitude and TE is the tıme to the echo

$26 \mathrm{~b}$ The twice-refocussed spin echo sequence [82] Gradient tımings are adjusted to minimize the effect of magnetic fields generated by eddy currents

27 (a) A gradient waveform, $G_{x}(t)$ (b) The first derivative of the gradient waveform, $d G_{x}(t) / d t$ (c) One of the induced eddy current terms, $g_{x}(t)$

(d) The intended and the actual gradient waveforms [98]

28 The figure contains a screen capture from the MR scanner showing the location of the navigator echo ROI ( $1 \mathrm{e}$ the blue box) positioned over the diaphragm for the 2D-PACE navigator echo respiratory triggering technique The yellow box indicates the volume that is to be imaged

29 The motion of the diaphragm during free breathing is represented by the green line The red boxes depict the acceptance windows that the respiratory algorithm has set for the trainıng period

210 The green line represents the motion of the diaphragm during free breathing in this screen capture from the MR scanner The yellow boxes represent the acceptance windows for image acquisition at the end of expiration

31 An image of a healthy liver (slice 11, volunteer 16) A HASTE sequence was used for this acquisition

32 The whole right side of the liver (WRL) ROI is shown in red and the periphery of the right side of the liver (PRL) ROI is shown in blue All ROIs have been drawn on anatomical images for a normal liver (volunteer 16)

33 Typical diffusion decays for the WRL-ROI (black) and the PRL-ROI (red) (volunteer 16)

34 An anatomical image for a normal liver (slice 19, volunteer 16) is shown on the lower left and $f$ (upper left), $D$ (upper right) and $D^{*}$ (lower right)-maps color overlaid onto the anatomical image, are shown as indicated The color table ranges for the maps are 1) 0 $100 \%$ for the $f$-map, 2) $(0-2) \mu \mathrm{m}^{2} / \mathrm{ms}$ for the $D$-map and 3) $(0-200)$ $\mu \mathrm{m}^{2} / \mathrm{ms}$ for the $D^{*}$-map 
$35 f$ values (mean $\pm \mathrm{SD}$ ) are shown for the WRL-ROI, the three 100-pixel ROIs and the PRL-ROI, averaged over all slices in each case The BH-protocol was used for volunteers 1 to 3 and the FB-protocol was used for volunteers 4 to 11 The horizontal lines show the mean over all volunteers for the PRL-ROI (red) and the WRL-ROI (black) For clarity, error bars are shown in a) for ROI1 only

$36 D$ values (mean $\pm \mathrm{SD}$ ) are shown for the WRL-ROI, the three 100pixel ROIs and the PRL-ROI, averaged over all slices in each case The BH-protocol was used for volunteers 1 to 3 and the FB-protocol was used for volunteers 4 to 11 The horizontal lines show the mean over all volunteers for the PRL-ROI (red) and the WRL-ROI (black) For clarity, error bars are shown in a) for ROI1 only

$37 D^{*}$ values (mean $\pm \mathrm{SD}$ ) for the WRL-ROI, the three 100-pixel ROIs and the PRL-ROI, averaged over all slices in each case The BHprotocol was used for volunteers 1 to 3 and the FB-protocol was used for volunteers 4 to 11 The horizontal lines show the mean over all volunteers for the PRL-ROI (red) and the WRL-ROI (black) For clarity, error bars are shown in a) for ROIl only

MRI image showing the anatomy of a healthy placenta (slice 19, patient 2) This image was acquired with a diffusion weighted echo planar sequence with $b=0$

42 The WP-ROI (red) and the three 100-pixel rectangular ROIs (blue) are shown on the image on the left and the ROIs drawn around the outer (red) and inner (blue) parts of the placenta are shown on the image on the right

43 Typical diffusion decays for the WP-ROI and a 100 pixel-ROI The decays shown are for the WP-ROI (black) and for one of the 100 pixel ROIs (red) shown in (slice 19, patient 2)

$44 \quad$ An anatomical image as well as $D, D^{*}$ and $f$-maps, color overlaid onto an anatomical image, are shown for a healthy pregnancy (slice 19, patient 2) Two areas of blood movement can clearly be seen on the $D^{*}$-map (shades of blue and green, respectively) and the $f$-map Areas where maternal blood enters the placenta from the spiral arteries can be seen and are indicated by the arrows on the $D^{*}$ map The color table ranges for the maps are a) $0-100 \%$ for the $f$-map, b) $(0-2)$ $\mu \mathrm{m}^{2} / \mathrm{ms}$ for the $D$-map and c) $(0-200) \mu \mathrm{m}^{2} / \mathrm{ms}$ for the $D^{*}$-map

45 Perfusion fraction, $f(\%)$, values plotted for each volunteer for 1$)$ the 100-pixel ROIs, 2) the whole placenta ROI (WP-ROI) and 3) the combined 100-pixel ROIs (U-ROI), averaged over all slices in each case For clanty, error bars are shown for ROI1 only The error bars 
correspond to the mean $\pm \mathrm{SD}$

$46 f$ values for each volunteer for the WP-ROI and the U-ROI, averaged over all shces in each case The error bars correspond to mean \pm SD

47 Diffusion coefficient values plotted for each volunteer for 1) the 100pixel ROIs, 2) the WP-ROI and 3) the U-ROI, averaged over all slices in each case For clarity, error bars are shown for ROIl only The error bars correspond to the mean $\pm \mathrm{SD}$

$48 D$ values plotted for each volunteer for the WP-ROI and the U-ROI, averaged over all slices in each case The error bars correspond to the mean \pm SD

49 Pseudo-diffusion coefficient values plotted for each volunteer for 1) the 100-pixel ROIs, 2) the WP-ROI and 3) the U-ROI, averaged over all slices in each case For clarity, error bars are shown for ROIl only The error bars correspond to the mean $\pm \mathrm{SD}$

$410 \quad D^{*}$ values plotted for each volunteer for the WP-ROI and the U-ROI, averaged over all slices in each case The error bars correspond to the mean $\pm \mathrm{SD}$

411 Typical behaviour for $f$ plotted as a function of slice number for the WP-ROI and the U-ROI The data are from patient 9 The error bars correspond to the mean $\pm \mathrm{SD}$

412 Typical behaviour for $D$ plotted as a function of slice number for the WP-ROI and the U-ROI The data are from patient 9 The error bars correspond to the mean \pm SD

413 Typical behaviour for $D^{*}$ plotted as a function of slice number for the WP-ROI and the U-ROI The data are from patient 9 The error bars correspond to the mean $\pm \mathrm{SD}$

$414 f$-values for the WP-ROI and the U-ROI plotted as a function of gestational age (GA) The error bars correspond to the mean \pm SD The black line shows the linear least squares fit to the WP-ROI data

$415 D$-values for the WP-ROI and the U-ROI plotted as a function of gestational age (GA) The error bars correspond to the mean \pm SD The black line shows the linear least squares fit to the WP-ROI data

$416 D^{*}$-values for the WP-ROI and the U-ROI plotted as a function of gestational age (GA) The error bars correspond to the mean \pm SD The black line shows the linear least squares fit to the WP-ROI data 
417 Signal attenuation versus $b$-value for inner ( $1 \mathrm{e}$ fetal side) and outer (1 e maternal side) placental regions for subject 1 , acquired using a breath hold technique The bi-exponential nature of the decays can clearly be seen

$418 f_{\text {out }}-f_{\text {in }}$ values for each volunteer (mean $\pm \mathrm{SD}$ ) $f_{\text {out }}=f_{\text {in }}$ is indicated by a solid line and negative values are shown in red

$419 D_{\text {out }}-D_{\text {in }}$ values for each volunteer (mean $\pm \mathrm{SD}$ ) $D_{\text {out }}=D_{\text {ln }}$ is indicated by a solid red line

$420 D_{o u t}^{*}-D_{m}^{*}$ values for each volunteer (mean $\pm \mathrm{SD}$ ) $D_{o u t}^{*}=D_{\text {on }}^{*}$ is indicated by a solid red line

51 Uncorrected simulated NCDs for several values of $\theta_{N}=A_{N} / \sigma_{g}$ for a 4element phased array and $\sigma_{g}=1$ The black, red, blue, green, purple and dark blue curves correspond to $A=00,05,10,20,30,40$, respectively

52 Simulated PDFs before ( $\Delta)$ and after $(\boldsymbol{\omega})$ the RB correction computed using exact values for $\mu_{\chi}$ and $\sigma_{g}$, for $N=1$ (red), $N=2$ (black) and $N=$ 4 (olive) elements and $\theta=05$ The vertical lines show the corresponding PDF means for the corrected (dashed) and uncorrected (solid) PDFs for $N=2$ Note that, for this case, the mean of the corrected PDF is very close to the expected value of 0707

Appendix E Simulated PDFs before and after the RNBC calculated using $\left\langle M_{N}\right\rangle_{\chi^{9}}$ and $\sigma_{g}$ for different $N$ element coils with $N=1,2,4,8$, and 16 


\section{List of Abbreviations}

$\begin{array}{ll}A D C & \text { Apparent Diffusion Coefficient } \\ \text { BH } & \text { Breath Hold } \\ \text { CV } & \text { Coefficient of Variation } \\ \text { CPMG } & \text { Car Purcell Meiboom Gill } \\ \text { DW } & \text { Diffusion Weighted } \\ \text { DWI } & \text { Diffusion Weighted Imaging } \\ \text { DTI } & \text { Diffusion Tensor Imaging } \\ D & \text { Free diffusion coefficient } \\ \text { EPI } & \text { Echo Planar Imaging }\end{array}$




\begin{tabular}{|c|c|}
\hline FB & Free Breathıng \\
\hline FGR & Fetal Growth Restriction \\
\hline FID & Free Induction Decay \\
\hline FOV & Field of View \\
\hline GA & Gestational Age \\
\hline GRAPPA & $\begin{array}{l}\text { Generalızed Autocalıbratıng Partıally Parallel Acquisıtıon - a } \\
\text { Parallel Imaging technıque performed in k-space }\end{array}$ \\
\hline HASTE & Half-Fourier Acquisition Single-shot Turbo Spin Echo \\
\hline IDEA & $\begin{array}{l}\text { Integrated Development Environment and Application - Siemens } \\
\text { sequence programming software package }\end{array}$ \\
\hline IPAT & integrated Parallel Acquisition Technıques \\
\hline IVIM & IntraVoxel Incoherent Motion \\
\hline LS & Least-Squares \\
\hline MR & Magnetic Resonance \\
\hline MRI & Magnetıc Resonance Imaging \\
\hline $\mathrm{NCD}$ & Non-central Chi Distribution \\
\hline NMR & Nuclear Magnetic Resonance \\
\hline NRT & Navigator echo Respiratory Triggerıng \\
\hline PDF & Probability Density Function \\
\hline PGSE & Pulsed Gradient Spın Echo \\
\hline PACE & Prospective Acquisition Correction \\
\hline PI & Parallel Imagıng \\
\hline PRL-ROI & Perıphery Right Liver Region of Interest \\
\hline QMR & Quantitative Magnetic Resonance \\
\hline RNB & Rectıfied Noise Bias \\
\hline RNBC & Rectıfied No1se Bias Correction \\
\hline
\end{tabular}




\begin{tabular}{|c|c|}
\hline RBF & Rectified Noise Floor \\
\hline RF & Radiofrequency \\
\hline RRF & Rotatıng reference Frame \\
\hline ROI & Region of Interest \\
\hline SD & Standard Deviation \\
\hline SS & Single Shot \\
\hline SNR & Signal-to-No1se Ratio \\
\hline SPAIR & Spectral Adıabatıc Inversion Recovery - a fat suppression technıque \\
\hline STIR & Short Time Inversion Recovery \\
\hline TE & Echo Time \\
\hline TR & Repetition Time \\
\hline$T_{1}$ & spin-lattıce relaxation time \\
\hline$T_{2}$ & spin-spın relaxation time \\
\hline $\mathrm{T}$ & absolute temperature \\
\hline TRSE & Twice Refocusing Spın Echo \\
\hline US & Ultrasound \\
\hline U-ROI & United Region of Interest \\
\hline WP-ROI & Whole Placenta Region of Interest \\
\hline WRL-ROI & Whole Right Liver Region of Interest \\
\hline
\end{tabular}


Chapter 1

\section{Introduction}




\subsection{Historical Introduction}

The phenomenon of nuclear magnetic resonance (NMR) was first discovered in 1946 by Bloch and Purcell who were awarded the Nobel Prize in physics in 1952 for their important discovery Between 1950 and 1970 NMR was used in physical and chemical molecular analysis Magnetic resonance imaging (MRI) began in 1973 when the research groups lead by Sir Peter Mansfield and Paul Lauterbur independently introduced the use of magnetic field gradients into the NMR procedure to obtain signal localization [1,2] In the beginning, back projection reconstruction methods, which were already well established in other areas of medical ımaging (e g Computer Assisted Tomography), were used to compute the MRI images In 1975 Ernst introduced the concepts of phase and frequency encoding and the use of the Fourier transform into MRI [3] This is the approach most commonly used today With contınuous developments in MRI hardware and information technology, fast dynamic MR acquisition has become possible, openıng up new diagnostic and interventional possibilities for MRI The 2003 Nobel Prize in medicine was awarded jointly to Sir Peter Mansfield and Paul Lauterbur for their important contributions to MRI and to medicine

\subsection{Introduction to Diffusion in Magnetic Resonance}

The method for sensitizing the magnetic resonance (MR) signal to the diffusion of water molecules was developed for NMR in 1965 by Stejskal and Tanner [4] and for MRI in 1985 by Le Bihan et al [5], who used essentially the same approach for sensitizing MR images to diffusion as was proposed by Stejskal and Tanner 
With the introduction of diffusion weighted (DW) MRI, studies of water transport in biological tissues in vivo became more popular Studies of molecular displacements over distances comparable to the cell size are expected to give insight into the geometry and spatial organization of the tissue as well as water exchange processes in each compartment in both normal and diseased tissue

Diffusion weighted MRI (DWI) has been very successful for the past twenty years Its main application has been in the evaluation of intracranial diseases such as cerebrovascular disease, depression, neurotoxicity and especially detecting early changes within the brain for patients with acute stroke, before any abnormality can be seen with other imaging techniques Although the first clinical Images of the brain were obtained in the $1980 \mathrm{~s}$, it was not possible to use DWI clinically until the 1990s when the major technological obstacles were overcome by a series of hardware improvements

In the early days of MRI, most scanners operated at relatively low magnetic field strengths in the range of 05 to 10 Tesla As a result, the signal intensity obtained was weak At that tıme, gradient strengths could not go beyond $10 \mathrm{mT} / \mathrm{m}$ due to hardware limitations and eddy currents caused image quality to be poor, at least by today's standards These weak gradients also meant that the MR signal could only be weakly sensitized to diffusion The absence of echo planar imaging (EPI), a very fast method for generatıng MR images, also made acquisition tımes for DWI unreasonably long and the acquired DW images were unreliable since they were very sensitive to macroscopic motion As for MR 
ımaging of the body, since there was no respiratory gatıng, the motion artifacts were atrocious and the images were worthless

These days much faster protocols are avallable and the entire brain can be imaged in a second DWI of extracranial sites, including the abdomen and the pelvis, is now possible A full set of DW measurements can be acquired in 1 to 5 minutes and no contrast agent is required The possibility of obtaining both qualitative and quantitative diffusion information as well as much higher quality DW images of the abdomen increased the interest in using DWI as an application for tumour assessment and/or disease diagnosis

Water diffusion in tissue is a very complex process The DW signal comes from the motion of water molecules in the extracellular space, the intracellular space and the intravascular space In the diffusion process, molecules move due to their thermal energy and collide with each other and with other structures in their local environment (e g cell membranes) These collisions cause the direction of motion for the molecules to change in a random fashion resulting in a molecular random translational motion on a microscopic scale which is well described as a random walk process Einstein was the first to correctly explain Brownian motion in terms of the random motion of particles due to their thermal energy in 1905 [6], thereby confirming the existence of atoms In a homogeneous liquid, the root mean square displacement, $\Delta r$, of the molecules over a given diffusion time, $t_{d}$, can be determined from the Einstein equation 


$$
\Delta r=\sqrt{2 d D t_{d}}
$$

where $d=1,2$ or 3 when diffusion takes place in 1,2 or 3-dimentions, respectively, and $D$ is a physical constant, called the self-diffusion coefficient, which characterizes the diffusion The root mean square displacement of water molecules that occurs during a diffusion measurement is estımated to be about $10 \mu \mathrm{m}$, which is comparable to the mean size of cells in the human body Thus, the information obtained from DWI can be expected to give information about the structure of the tissue at the cellular scale This is one of the reasons why DWI has been seen as a potential tool for imaging tumour microcirculation (before and after treatment) in the abdomen

The MR signal is sensitized to diffusion in a specific direction by inserting appropriate magnetıc field gradient pulses into an MR pulse sequence Images with different degrees of diffusion weighting can be obtained if various gradient pulse amplitudes are applied The direction in which the MR sequence is sensitive to diffusion is determıned by the orientation of the applied gradient pulses In pure liquids, the signal decay, as a function of the diffusion weighting, is exponential and can be expressed as

$$
S(b) / S_{0}=\exp (-b D)
$$

where $b$ is a measure of diffusion sensitization and $S$ and $S_{0}$ are the signals at $b$ and $b=0$, respectively The $b$-value depends on both the amplitude and the duration of the diffusion gradients The overall diffusion signal measured across an image voxel results from the integration of all of the microscopic displacements of the water molecules present in the voxel 


\subsubsection{Intravoxel Incoherent Motion}

"I had a fuzzy intultion that perhaps molecular diffusion measurement would result in low values in solld tumors because of molecular movement restriction while diffusion would be somewhat enhanced in flowing blood" Dens Le Bihan [7]

In the mid 1980s, Le Bihan proposed that, in addition to diffusion, the flow of water molecules in blood in capillary segments that are onented in a pseudorandom manner can also be treated as a random walk process and he presented a mathematical model based on diffusion theory to explain his results This is known as the IntraVoxel Incoherent Motion (IVIM) model The corresponding diffusion and pseudo-diffusion coefficients differ only by about one order of magnitude $(D$, the molecular diffusion coefficient of water in tissues, is about $1 \mu^{2} / \mathrm{ms}$, while $D^{*}$, the pseudo-diffusion coefficient associated with capillary blood flow, is about $10 \mu^{2} / \mathrm{ms}$ in the brain [8] and $70 \mu^{2} / \mathrm{s}$ in the liver [9] The proximity of these values allows $D$ and $D^{*}$ to be evaluated together with the same diffusion MRI sequence It also means that DWI images are prone to contamination by blood microcirculation effects

The IVIM model is considered in substantially more detail in Chapter 2 


\subsection{Abdominal Imaging Literature Review}

The " $A D C$ concept" has been generally adopted in the medical MR literature because it was obvious that the diffusion coefficient, $D$, should be replaced for tıssue studies by a "global" parameter, known in the MR literature, as the apparent diffusion coefficient or the $A D C$ The measured diffusion coefficient is not a true measure of 'intrinsic' diffusion, it represents the complex molecular displacements that occur in biological tissues due to interactions of water molecules with cellular structures and processes over a given diffusion time In a voxel that contains thousands of cells and tissue components, the diffusion signal is given by the average of many different effects and microscopic environments With the IVIM model discussed above the measured diffusion decay is considered to be bi-exponential, with one component corresponding to diffusion and the other to the pseudo-diffusion process associated with the water in the microvasculature When the $A D C$ approach is used, the decay is inherently assumed to be monoexponential where the $A D C$ is the decay constant In practice, the measured diffusion decay fits very well to a bi-exponential However, for some tissues, such as the brain, the contribution from the second process is quite small and the deviation from exponentiality is relatively minor For these tissues the $A D C$ values can be quite useful For more vascular organs, such as the liver or the placenta, the diffusion decay is strongly bi-exponential and, if the $A D C$ approach 1s used, it must be done with caution For such tissues the IVIM model is preferable 
Numerous studies of DWI for liver have recently emerged, including for liver lesion detection and characterization [10-28], for the diagnosis of liver fibrosis or currhosis [29-33] and for liver tumour treatment response [34-41] Unfortunately, the reported $A D C$ values vary over a very wide range $(300 \%$ variation) for both normal liver and for focal hepatic lesions $[42,43]$ This variation is probably related to different sets of $b$-values used in the protocol (since the decay deviates strongly from the assumed exponential decay), different algorthms used for image analysis, the stage of the tumour when investigated [43] and different experimental set-ups used for ımage acquisition Most of these studies mentioned above have used breath hold single shot (SS) EPI to acquire DW images, using various $b$-values These sequences are fast but they suffer from poor image quality when higher $b$-values are used These results demonstrate that there is a need for the implementation and validation of more reliable data acquisition techniques and image analysis tools for DWI in the abdomen

The development of parallel imaging has been very important for abdominal and pelvic MRI because it enables the use of shorter echo times and reduced echo train length, resulting in a faster acquisition of images with a good signal to noise ratio (SNR) that are relatively undegraded by motion and other artıfacts Parallel ımaging [44,45] and pulse triggering [46] have been proposed to improve diffusion image quality and the precision of $A D C$ measurements

Respiratory triggering is another method that has been used to improve DW image qualıty, it is a technıque that avoids motion artıfacts by synchronızıng image acquisition with the patient's breathing cycle and by doing the data 
acquisition at the end of the expiration phase [47-55] Various techniques have been used for respiratory triggering bellow systems in combination with a strain gauge, temperature monitoring using face masks, and elastıc breathing belts, as well as navigator echoes The major advantages of navigator echoes are that no additional hardware is needed, patient set up is easier and this technique works much better than the others

A few recent studies have reported the use of respiratory triggered liver DWI [47-49] where the triggering was done without using navigator echoes Gourtsoyiann et al [48] assessed the ut1lity of the $A D C$ values obtained with a non-navigator echo respiratory triggered SS EPI DWI sequence $(n=38)$ for characterization of liver lesions and found sensitivity and specificity values of $100 \%$ for the diagnosis of malignancy

There are only a few studies in the literature that use the IVIM model for etther of the organs investigated for this thesis, there are two for the liver $[9,12]$ and two for the placenta [56-58] Detalled comparisons of these results with ours are found in Chapter 3 for the liver and Chapter 4 for the placenta

\subsubsection{IVIM Perfusion versus Classical Perfusion}

Throughout this thesis the terms perfusion, perfusion coefficient and perfusion fraction are used In the classical physiology literature perfusion refers to the delivery of nutrients and the removal of waste products from a tissue and is expressed in millilitres per minute per 100 grams of tissue The "perfusion" measurements reported in this thesis do not measure perfusion in this classical 
sense, although they are closely related [59] Classical perfusion in a given tissue is determined primarily by blood flow to the tissue and in the microvasculature of the tissue The "perfusion" parameters reported here are determined by the microcirculation in the tissue of interest and are thus very closely related to classical perfusion but are not exactly the same By "perfusion", we are really referring to the microcirculation of blood in the tissue The "perfusion coefficient", which is equated with the pseudo-diffusion coefficient introduced above, is an indirect measure of the movement of the blood in the tissue microvasculature and has units of a diffusion coefficient $\left(1 \mathrm{e} \mu^{2} / \mathrm{ms}\right)$ The "perfusion fraction" refers to the fraction of the MR signal that is associated with the "perfusion coefficient" In other words, it gives the fraction of the MR signal associated with blood moving in the microvasculature of the tissue See Section 233 for further detalls The decision to use this terminology was made for consistency with related work in the MRI literature

\subsection{Thesis Outline}

DWI is sensitive to microscopic motion in biological tissues, including self diffusion of water molecules and the microcirculation of blood in the capillary network of the tissue It is overly simplistic to assume that the attenuation of the diffusion signal intensity as the $b$-value increases is a simple exponential decay in tissue, particularly in highly vascular tissues such as the liver or the placenta In this thesis the aim was to investigate water diffusion in these two highly vascular 
organs using the IVIM model, a model that more closely represents the hemodynamics in the tissue of interest Organs such as the placenta and the liver have not been properly investigated in the past due in part to hardware limitations and/or poor analysis tools With new advancements in the hardware technology, a re-evaluation of the models used to describe the DWI decay data is required

To increase the image quality of the acquired DWI ımages as well as the precision and accuracy of the diffusion measurements relative to previously reported studies for both organs, the experimental procedure was enhanced by usıng 1) parallel ımagıng, 2) navigator echo respıratory triggerıng, 3) a double echo diffusion sequence that inherently compensates for the effects of eddy currents 4) SPAIR fat suppression and 5) a superior approach to image analysis The experıments reported here represent the first studies to give results for abdominal DWI performed with these enhancements and analyzed using the IVIM model $[60,61]$ We believe that, for both of these organs, the IVIM parameters reported here are considerably more reliable than those previously reported in the literature

The IVIM parameters discussed in this thesis are presented for 1) a region of interest (ROI) covering the whole right side of the liver or the entire placenta, 2) 3 separate 100-pixel square ROIs located around the periphery of the right side of the liver or placed carefully across the placenta to avoid big blood vessels or turbulent flow, 3) an ROI corresponding to the union of the three 100-pixel ROIs and 4) a pixel-by-pixel analysis from which parametric maps were generated 
The information obtained from these different choices and their relative merits and importance are discussed

In Chapter 2, a review of all of the relevant background for the thesis is given In Chapters 3 and 4, diffusion decay measurements for normal liver and healthy placenta in human volunteers in vivo are presented and discussed The experımental results are explained using the b1-exponential IVIM model One potential limitation of the IVIM approach, where the full diffusion decay must be measured, is that rectified noise bias can distort the decay for large $b$-values where the SNR can sometımes be quite low (e $\mathrm{g}$ when the voxel size is small) In Chapter 5 a useful technique for removing this noise bias from the pixel intensities of magnitude MR images is presented for images acquired using phased array coils The thesis is finalized with Chapter 6, General Conclusion 


\section{Chapter 2}

\section{Theory and Background}




\subsection{Introduction}

In this chapter, the basic physical principles of nuclear magnetic resonance (NMR) and diffusion are explained in order to facilitate a better understanding of the studies presented in the following chapters Section 22 is dedicated to the presentation of NMR phenomenology Section 23 describes diffusion in magnetic resonance imaging (MRI) with particular emphasis on the Intravoxel Incoherent Motion (IVIM) model The PACE respiratory triggerıng technique is explained in section 24 In the two final sections of this chapter, introductions to the anatomy and physiology of the placenta and the liver are given The principles behind the MR imaging experımental methodology used in the placenta and liver studies are also introduced in section 23 and 24

\subsection{Principles of Nuclear Magnetic Resonance}

\subsubsection{The Nuclear Magnetic Moment in a Static Magnetic Field}

Magnetic resonance (MR) phenomena can be observed in isotopes with non-zero nuclear spin as a result of the fact that they inherently possess both a magnetic moment and angular momentum [62] Nucle1 are composed of nucleons (1 e protons and neutrons), each of which has a spin of $1 / 2$ The coupling of the spins of the nucleons that form a particular nucleus determine the nuclear spin, $I$, where $I$ may be integral or half-integral ( $1 \mathrm{e} I=n / 2$ with $n$ being an integer) As a result, the total angular momentum of the nucleus, $\boldsymbol{J}$, can be written as [63]

$$
\boldsymbol{J}=\hbar \boldsymbol{I},
$$


where $\hbar$ is Dirac's constant and $I$ is the dimensionless nuclear spın angular momentum operator $I^{2}$ has eigenvalues $I(I+1)$ and for ${ }^{1} \mathrm{H}, I=1 / 2$ Note that throughout this thesis, a bold font will be used to indicate vector quantities

The total nuclear magnetıc moment, $\boldsymbol{\mu}$, and $\boldsymbol{J}$ are parallel vectors that are related by $\gamma$, a scalar known as the gyromagnetic ratio, which is a constant for a given nucleus

$$
\boldsymbol{\mu}=\boldsymbol{\gamma} \boldsymbol{J}
$$

The gyromagnetic ratio for hydrogen, $\gamma_{\mathrm{I}_{\mathrm{H}}}$ is equal to $2675 \times 10^{8} \mathrm{rad} \mathrm{T}^{-1} \mathrm{~s}^{-1}$ [64]

The interaction of $\boldsymbol{\mu}$ with $\boldsymbol{B}_{0}=B_{0} \boldsymbol{k}$ causes a splitting of the ground state energy level This is known as the Zeeman effect and is described by the Hamiltonian [64]

$$
H=-\boldsymbol{\mu} \boldsymbol{B}_{0}=-\gamma \hbar I_{z} B_{0}
$$

The allowed energies, which correspond to the eigenvalues of this Hamiltonian, are given by

$$
E=-\gamma \hbar B_{0} m
$$

where $m=-I,-I+1,, I-1, I$ are the eigenvalues of $I_{z}$ [64] The energy difference between adjacent levels is

$$
\Delta E=\gamma \hbar B_{0}
$$

An externally applıed statıc magnetıc field, $\boldsymbol{B}_{0}$, will exert a torque on $\boldsymbol{\mu}$ As a result, $\boldsymbol{\mu}$ will change, in a short tıme $d t$, by an amount $d \boldsymbol{\mu}$ corresponding to a rotation through an angle $d \theta$ in the plane perpendicular to $\boldsymbol{B}_{0}($ see Fig 21 ) This precessional motion of $\boldsymbol{\mu}$ represents the dynamics of any 1solated magnetic 
moment in a static magnetic field in both classical and quantum mechanical approaches to NMR [64] The frequency of precession, $\omega_{0}$, is known as the Larmor frequency and is directly proportional to $\boldsymbol{B}_{0}$

$$
\omega_{0}=-\gamma \boldsymbol{B}_{0}
$$

This is a fundamental concept in MRI For most clinical MRI scanners $v_{0}=\omega_{0} / 2 \pi$ 1s between about 40 and $130 \mathrm{MHz}$, correspondıng to magnetıc field strengths of 1 $\mathrm{T}$ to $3 \mathrm{~T}$ [64] If $\mathrm{Eq}(26)$ is inserted into $\mathrm{Eq}(25)$ the energy separation between adjacent Zeeman energy levels, $\Delta E$, becomes

$$
\Delta E=\hbar \omega_{0}
$$

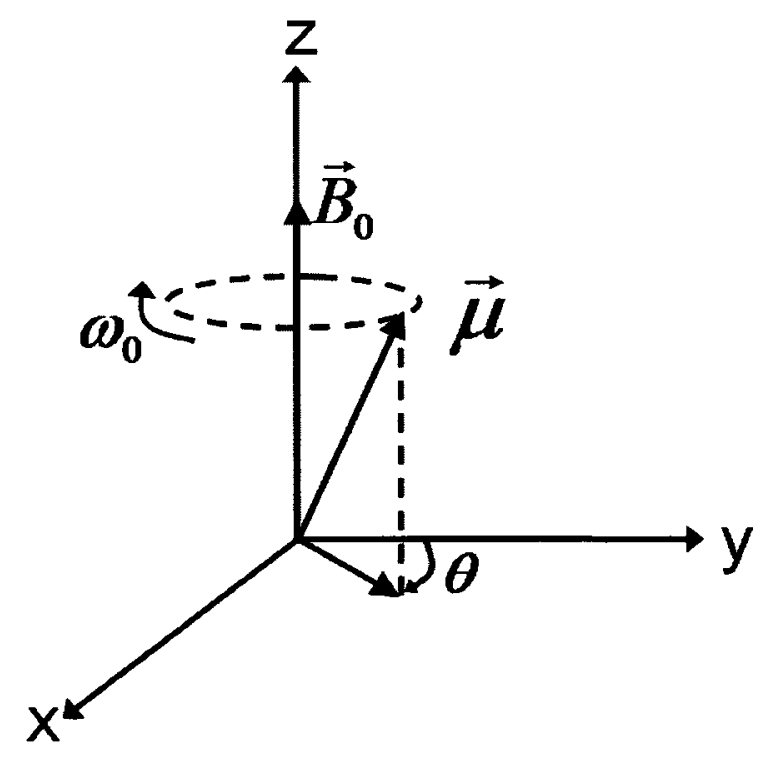

Fig. 2.1. Precession of $\boldsymbol{\mu}$ about $\boldsymbol{B}_{0}$ at the Larmor angular frequency $\omega_{0}=\gamma \boldsymbol{B}_{0}$ The angle $\theta$ represents the phase of the precession 


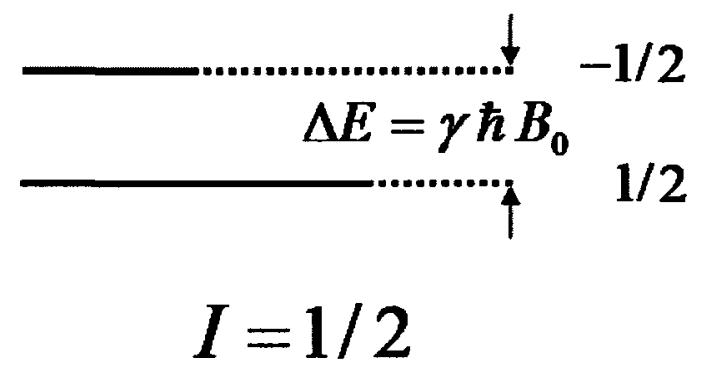

Fig. 2.2. Energy level diagram for a nuclear spın with $I=1 / 2$ in a static magnetic field, $\boldsymbol{B}_{0} \Delta E=\gamma \hbar B_{0}$ represents the energy difference between levels separated by $\Delta m= \pm 1$ The bold line schematıcally represents the relative population in each state for an ensemble of spins at thermal equilibrium

\subsubsection{Nuclear Spin Energy Level Population in a Static Magnetic Field}

For the case of a population of nuclear spins in the presence of a static magnetic field, $\boldsymbol{B}_{0}$, at thermal equilibrium, one can use Boltzmann statistics to find the ratio between the number of nucle1 in the lower energy state, $n_{-}$, and the number of nucle1 in the upper energy state, $n_{+}[63]$

$$
\frac{n_{-}}{n_{+}}=\exp \left(\frac{\Delta E}{k T}\right),
$$

where $k$ is the Boltzmann constant, $T$ is the absolute temperature of the spin system and $\Delta E$ represents the energy difference between Zeeman levels defined by Eq (2 5) A Zeeman energy level diagram for $I=1 / 2$ nuclear spins and the relative population of each state is shown schematically in Fig 22

For a population of $N$ nuclei with spin $I=1 / 2$, the polarization $P$ is represented by the fractional excess of nucle1 in the lower energy state [63] 


$$
P=\frac{n_{-}-n_{+}}{N}=\frac{n_{-}-n_{+}}{n_{-}+n_{+}}
$$

Using Eq (2 8) the polarization $P$ can be rewritten as

$$
P=\tanh \left(\frac{\Delta E}{2 k T}\right)
$$

The alignment of the spins in a static magnetic field can also be expressed in terms of the magnetization, $\boldsymbol{M}$, which is defined as

$$
\boldsymbol{M}=\sum_{i=1}^{N} \boldsymbol{\mu}_{\imath},
$$

where the $\boldsymbol{\mu}_{\imath}$ represent the magnetic moments of individual nuclei In equilibrium, there is a small surplus of nuclear magnetıc moments with $\mathrm{z}$ components aligned parallel to $\boldsymbol{B}_{0}$ as opposed to ant1-parallel However, the sum of all magnetic moment projections onto the plane perpendicular to $\boldsymbol{B}_{0}$ (1 $\mathrm{e}$ the transverse plane) is zero Therefore, the magnitude of the magnetization, $M$, for spin-1/2 nucleı, can be expressed as

$$
M=|\boldsymbol{M}|=\left|\sum_{\imath=1}^{N} \boldsymbol{\mu}_{\imath}\right|=\frac{1}{2} N \gamma \hbar P
$$

The measured signal in MR is proportional to $M$

The equation of motion of the macroscopic magnetization is obtained by equating the torque exerted on $\boldsymbol{M}$ by $\boldsymbol{B}$ with the rate of change of the magnetization [64]

$$
\frac{d \boldsymbol{M}}{d t}=\gamma \boldsymbol{M} \times \boldsymbol{B}
$$




\subsubsection{Effect of RF Pulses and Magnetic Field Gradients on $\boldsymbol{M}$}

The concept of the rotating reference frame (RRF) [63] can be used to better understand the effect of a radıo frequency (RF) magnetıc field on the bulk magnetization of the spin population Since, in the presence of a static magnetic field, Larmor precession always occurs, the use of a RRF which rotates in the transverse plane at the same angular frequency, $\omega$, as the RF magnetic field, simplifies the analysis

By applyıng a tıme-varying magnetic field, $\boldsymbol{B}_{1}$, with RF carrier frequency $\omega \approx \omega_{0}$ in a direction perpendicular to $\boldsymbol{B}_{0}$ (most efficient excitation), the nuclear spins are excited and the magnetic resonance effect can be measured [63] The effective magnetic field, $\boldsymbol{B}_{\text {eff }}$, experienced by the spins in the RRF is given by [63]

$$
\boldsymbol{B}_{\text {eff }}=\boldsymbol{B}_{1}+\boldsymbol{B}_{0}-\boldsymbol{\omega} / \gamma
$$

Fig 23 shows the difference between the effect of the total magnetic field in the laboratory frame $\left(\boldsymbol{B}_{\text {total }}\right)$ and in the $\operatorname{RRF}\left(\boldsymbol{B}_{\text {eff }}\right)$

In MRI, $\boldsymbol{B}_{1}$ is applied as an RF pulse of duration $t_{p}$ Magnetization initially aligned along the direction of $\boldsymbol{B}_{0}$ will change its orientation due to the RF pulse (see Fig 2 4) In the RRF ( $x^{\prime}, y^{\prime}, z^{\prime}$ ), on resonance (1 e $\omega=\omega_{0}$ ), the magnetization, $\boldsymbol{M}$, is tipped towards the $x^{\prime} y^{\prime}$-plane, the plane perpendicular to $\boldsymbol{B}_{0}$, by the RF pulse [63] The angle of rotation or the tip angle, $\alpha$, depends on the duration of the pulse, $t_{p}, B_{1}$ and $\gamma$ 


$$
\alpha=\gamma \int_{0}^{t_{p}} B_{1}(t) d t
$$

If $B_{1}$ is uniform in the region of interest, $\alpha$ can be calculated using Eq (2 15)

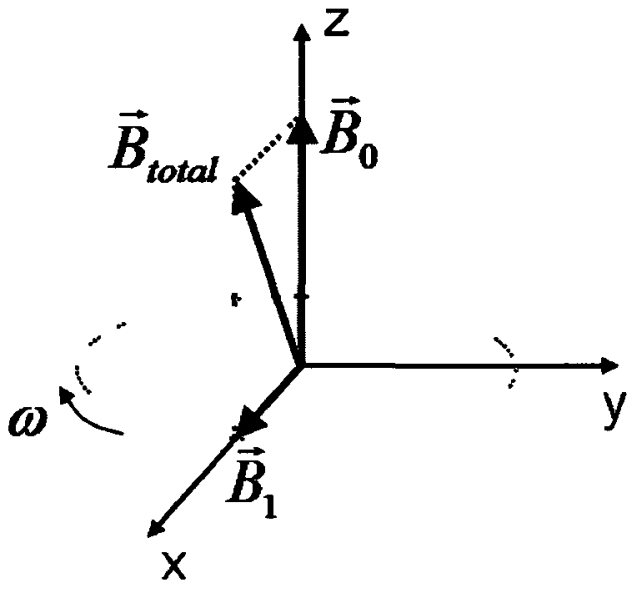

(a)

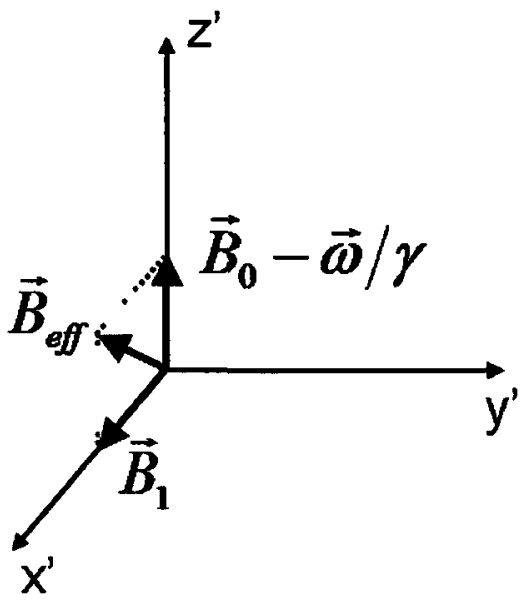

(b)

Fig. 2.3. (a) The total magnetic field, $\boldsymbol{B}_{\text {total }}$, in the laboratory frame $(x, y, z)$ (b) The effective field, $\boldsymbol{B}_{\text {eff }}$, in the RRF (x', y', $z^{\prime}$ ) which rotates at angular frequency $\omega$

\subsubsection{The Bloch Equations and Relaxation}

There are two types of relaxation involved in the return to the "thermal" equlibrium state of a spin population that was previously excited by a resonant RF pulse The first relaxation process is called longitudinal or spin-lattıce relaxation In this process there is an exchange of energy between the spin system and the lattice ( $\mathrm{e}$ the surrounding thermal reservoir) The return of the longitudinal magnetization, $M_{2}$, to its equilibrium value, $M_{0}$, is shown graphically in Fig 25 (a) as an exponential growth and can be described using the 


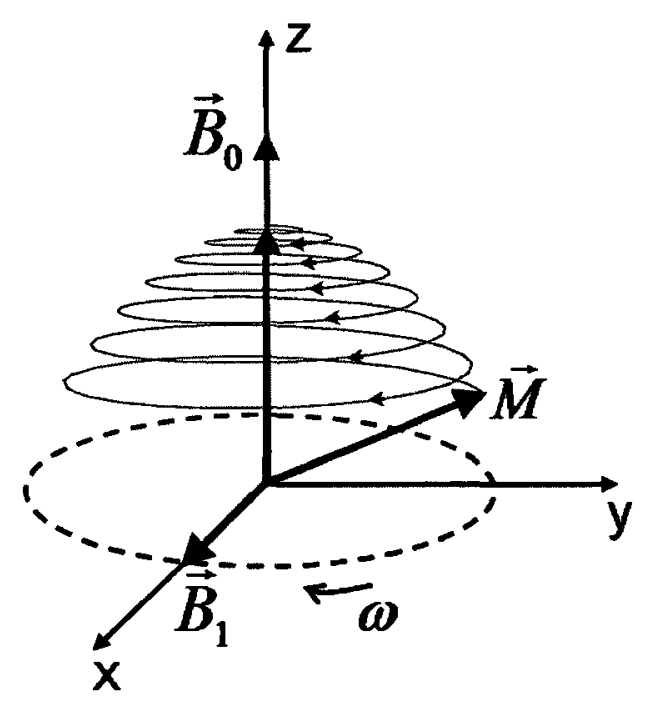

(a)

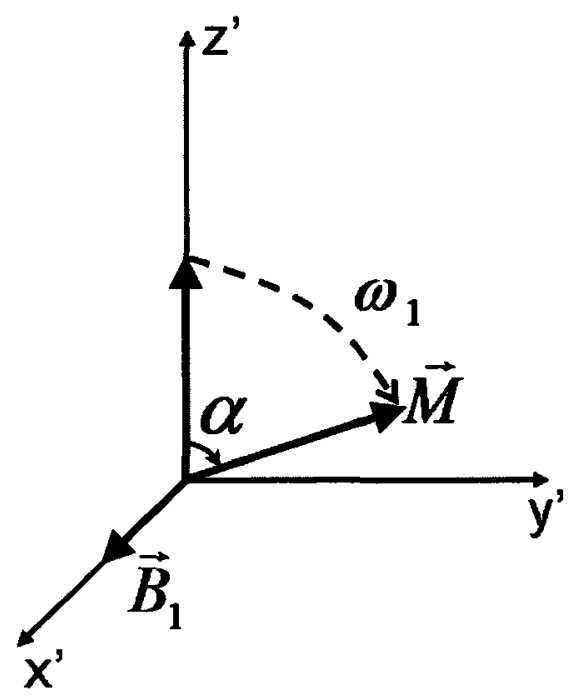

(b)

Fig. 2.4. (a) The trajectory of $M$ in the laboratory frame (x, y, z) in the presence of both 1) a longitudinal static magnetic field, $\boldsymbol{B}_{0}$, and 2) a transverse rotating field $\boldsymbol{B}_{1}$ On resonance $\left(\omega=\omega_{0}\right), \boldsymbol{M}$ precesses simultaneously about both $\boldsymbol{B}_{0}$, at frequency $\omega_{0}$, and $\boldsymbol{B}_{1}$, at frequency $\omega_{1}$, where $\omega_{0} \gg \omega_{1}$ (b) The trajectory of $\boldsymbol{M}$ in the RRF (', $\mathrm{y}^{\prime}, \mathrm{z}^{\prime}$ ) on resonance For this case the longitudinal effective magnetic field will be zero

equation [64]

$$
\frac{d M_{z}}{d t}=-\frac{M_{z}-M_{0}}{T_{1}}
$$

which has the solution

$$
M_{z}(t)=M_{z}(0) \exp \left(-t / T_{1}\right)+M_{0}\left(1-\exp \left(-t / T_{1}\right)\right)
$$

$T_{1}$, which is referred to as the longitudinal, or spin-lattice, relaxation tıme, is typically in the range 01 to about $3 \mathrm{~s}$ for water protons in tissues at clinical MRI field strengths 


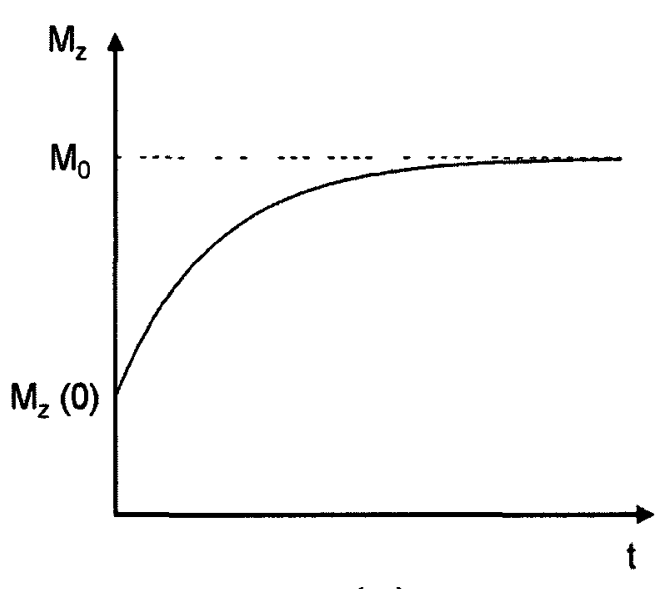

(a)

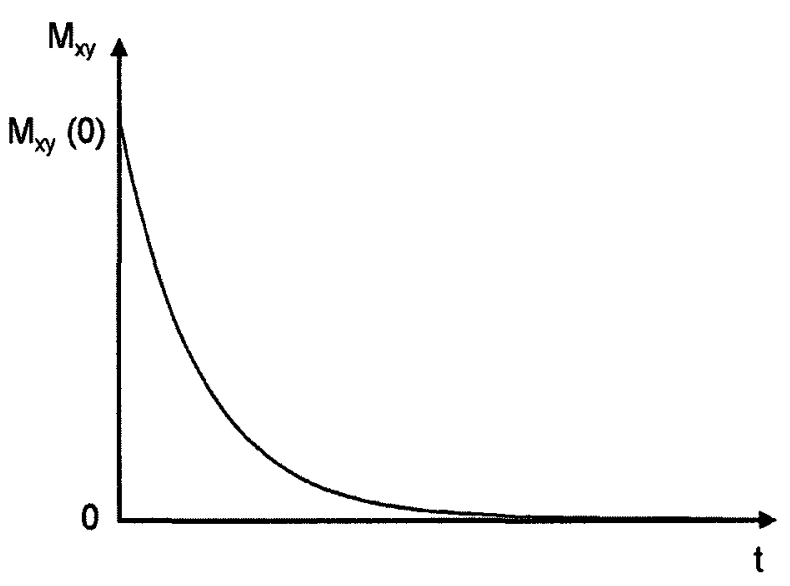

(b)

Fig. 2.5. Diagrams of (a) longitudinal magnetization $M_{z}$ and (b) transverse magnetization $M_{x y}$ relaxation as a function of time, $t$

The return of the longitudinal magnetization to thermal equilibrium is a dissipative process with part of the absorbed energy being transferred to the lattice in the form of heat

The second form of relaxation is the transverse, or spin-spin, relaxation process which is represented graphically in Fig 25 (b) as an exponentıal decay In the transverse magnetization there is phase coherence among the nuclear spin states immediately after the RF pulse Interactions among the spins disturb this spin coherence and the transverse magnetization decays as a result [64] The equation describing this phenomenon, in the rotatıng reference frame (on resonance), is

$$
\frac{d M_{x y^{\prime}}}{d t}=\frac{-M_{x^{\prime} y^{\prime}}}{T_{2}}
$$

which has the solution 


$$
M_{x^{\prime} y^{\prime}}(t)=M_{x^{\prime} y^{\prime}}(0) \exp \left(-t / T_{2}\right)
$$

$T_{2}$ is called the transverse or spin-spin relaxation time

The loss of coherence is observed not only due to spin-spin interactions In the frequency domain, the resonance peaks will broaden if the local static field varies with position In this case, the signal in the time domain decays with the apparent transverse relaxation tıme, $T_{2}^{*}$ Diffusion and chemical exchange can also influence transverse relaxation [64]

The local static field experienced by a nuclear spin is affected by 1) $B_{0}$ inhomogeneities related to the magnet design, 2) magnetıc susceptıbility differences related to the sample's intrinsic magnetic properties, and 3) electronic shielding effects The use of active or passive shimming can dimınish the effect of $B_{0}$ inhomogeneity The magnetic field around the nucleus depends on the surrounding environment (1 e electronic shells, chemical bonds, etc) This is why, for a specific static field, $B_{0}$, different substances have slightly different resonance frequencies In MR, the resonance frequency for a particular substance, $v_{S}$, is often expressed as the difference between $v_{S}$ and the resonance frequency, $v_{R}$, of a reference material This frequency difference is an intrinsic property of the material known as its chemical shift, $\delta$, and it is normally given in parts per million (ppm) to elıminate the dependence on the static magnetic field [64]

$$
\delta=\frac{v_{S}-v_{R}}{v_{0}} 10^{6},
$$

where $v_{0}$ ss the operating frequency of the scanner 
In practice, measurements of $T_{2}$ are traditionally performed using a CarrPurcell-Meıboom-Gill (CPMG) pulse sequence $[65,66]$ which consists of a $90^{\circ}$ $\mathrm{RF}$ pulse followed by a set of equally spaced $180^{\circ} \mathrm{RF}$ pulses that are phase shifted by $90^{\circ}$ with respect to the $90^{\circ}$ pulse to avold complications resulting from inaccurate refocusing pulse flip angles [64]

The effects of chemical exchange, diffusion, chemical shift and susceptıbility differences can be only partially corrected using pulse sequences such as CPMG These effects represent some of the challenges faced in the MR experimental world and at the same tıme they limit the resolution and signal-to noise ratıo (SNR) in MRI [64]

In the rotatıng reference frame, a single vector equation which describes the tıme dependence of the magnetization vector

$$
\frac{d \boldsymbol{M}}{d t}=\boldsymbol{M} \times \gamma \boldsymbol{B}_{e f f}-\frac{M_{x^{\prime}} \boldsymbol{x}^{\prime}+M_{y^{\prime}} \boldsymbol{y}^{\prime}}{T_{2}}-\frac{\left(M_{z^{\prime}}-M_{0}\right) z^{\prime}}{T_{1}},
$$

can be generated by combinıng Eqs (213), (2 16) and (2 18), where $x^{\prime}, y^{\prime}$ and $z^{\prime}$ are the unit vectors in the $x^{\prime}, y^{\prime}$ and $z^{\prime}$ directions, respectively This equation, known as Bloch's equation, gives a simplified way of understanding the relaxation effects and is very important in describing MR phenomena By combining this equation with the classical diffusion equation the Bloch-Torrey equation can be formed [73] 


\subsection{Diffusion}

\subsubsection{Diffusion Theory}

\subsubsection{Fick's law and Einstein's equation}

MRI provides a good, noninvasive method for the measurement of the translational motion of water molecules in tissue In 1905, Einstein showed that the random motion of spherical particles suspended in a fluid, a phenomenon known as Brownian motion, was the result of the particles' thermal energy [6] This microscopic random translational motion is also known as molecular diffusion

The net displacement, $\boldsymbol{r}(t)-\boldsymbol{r}_{0}$, of a particle (e $\mathrm{g}$ a water molecule) from its initial position, $r_{0}$, due to Brownian motion, is time dependant The particle flux, $J(r, t)$, in an isotropic medium is, according to Fick's first law of diffusion, proportional to the partıcle concentration gradient [67]

$$
J(\boldsymbol{r}, t)=-D \nabla c(\boldsymbol{r}, t)
$$

where $D$ is the diffusion coefficient and the concentration, $c(r, t)$, is the number of particles per unit volume at a given position, $\boldsymbol{r}$, and time is represented by $t$ The negative sign indicates that the direction of the net motion is from a larger to a smaller concentration

The continuity theorem [67]

$$
\nabla \boldsymbol{J}(\boldsymbol{r}, t)+\frac{\partial c(\boldsymbol{r}, t)}{\partial t}=0
$$

also applies to this situation from conservation of mass considerations [67] 
Combinıng Eqs (2 22) and (2 23) gives Fick's second law of diffusion

$$
\nabla^{2} c(\boldsymbol{r}, t)-\frac{1}{D} \frac{\partial c(\boldsymbol{r}, t)}{\partial t}=0
$$

This equation is also known as the diffusion equation The solution of the diffusion equation in an unbounded isotropic medium is [67]

$$
c(\boldsymbol{r}, t)=\frac{N}{(4 \pi D t)^{3 / 2}} \exp \left[\frac{-\left(\boldsymbol{r}-\boldsymbol{r}_{0}\right)^{2}}{4 D t}\right],
$$

where $N$ is the total number of particles in the system It can be seen from Eq (225) that the particle concentration at time $t, c(r, t)$, depends only on the net displacement $\boldsymbol{r}(t)-\boldsymbol{r}_{0}$, at time $t$ and not its actual position at time $t$ This is an intrinsic property of Brownian motion Such a displacement causes an attenuation of the MR signal Therefore, if the attenuation of the MR signal can be measured, the mean displacement of the partıcles ( $1 \mathrm{e}$ the nuclear spins) can be evaluated

Einstein showed that, for an ensemble of particles, the diffusion coefficient measured in the non-equilibrium experiment described above is proportional to the variance of $P\left(r_{0} \mid \boldsymbol{r}, t\right)$, the conditional probability for a diffusing particle, initially at position $r_{0}$, to be found at position $r$ at time $t$ For free diffusion, this conditional probability distribution follows the same diffusion equation as the particle concentration and it can be shown that [67]

$$
<\left(r-r_{0}\right)^{2}>=2 d D t_{d}
$$

where $d=1,2$ or 3 represents the dimensionality of the system and $t_{d}$ is called the diffusion time Eq (2 26), known as Einstein's equation, shows that the meansquared displacement of the diffusing molecules increases linearly with $t_{d}$ 
So far the mathematical description of diffusion presented here assumes that the diffusion process is isotropic and can therefore be described by the isotropic diffusion coefficient $D$, which is a scalar For water it can be shown that $[6,66]$

$$
D=\mu_{p} k_{B} T,
$$

where $D$ describes the rate at which molecules spread out in a fluid medium, $\mu_{p}$ is the mobility of the particles (related to particle size and viscosity), $k_{B}$ is the Boltzmann constant and $T$ is the absolute temperature

It can also be shown that $D$ is given by [69]

$$
D=\frac{1}{6} \bar{l} \bar{v}=\frac{1}{6} \bar{l}^{2} / \bar{t},
$$

where $\bar{l}, \bar{v}$ and $\bar{t}$ are the average displacement, average speed and average duration, respectively, for the steps of the random walk process

\subsubsection{Diffusion and NMR}

Fifty years after Einsteın's discovery, Hahn [70] and Carr and Purcell [71] developed a method to measure the self-diffusion coefficient, $D$, using NMR Stejskal and Tanner modified this approach by introducing pulsed magnetic field gradients to achieve a more accurate measurement [4] This method is still widely used in diffusion-weighted MRI to measure the average displacement of water molecules in tissue

The Pulsed-Gradient Spin Echo (PGSE) sequence, sometımes also referred to as the Stejskal Tanner sequence, is shown schematıcally in Fig $26 \mathrm{a}$ It consists of a standard spin echo sequence with two additional identical diffusion- 
weightıng gradient pulses added, one before the $180^{\circ} \mathrm{RF}$ refocusing pulse and one after it

For the purposes of this discussion the gradient pulses will be assumed to be very short, such that 1 ) the particle motion during each gradient pulse is negligible and 11) their duration, $\delta$, is much smaller than their separation, $\Delta$ This last condition is known as the narrow pulse approximation [4]

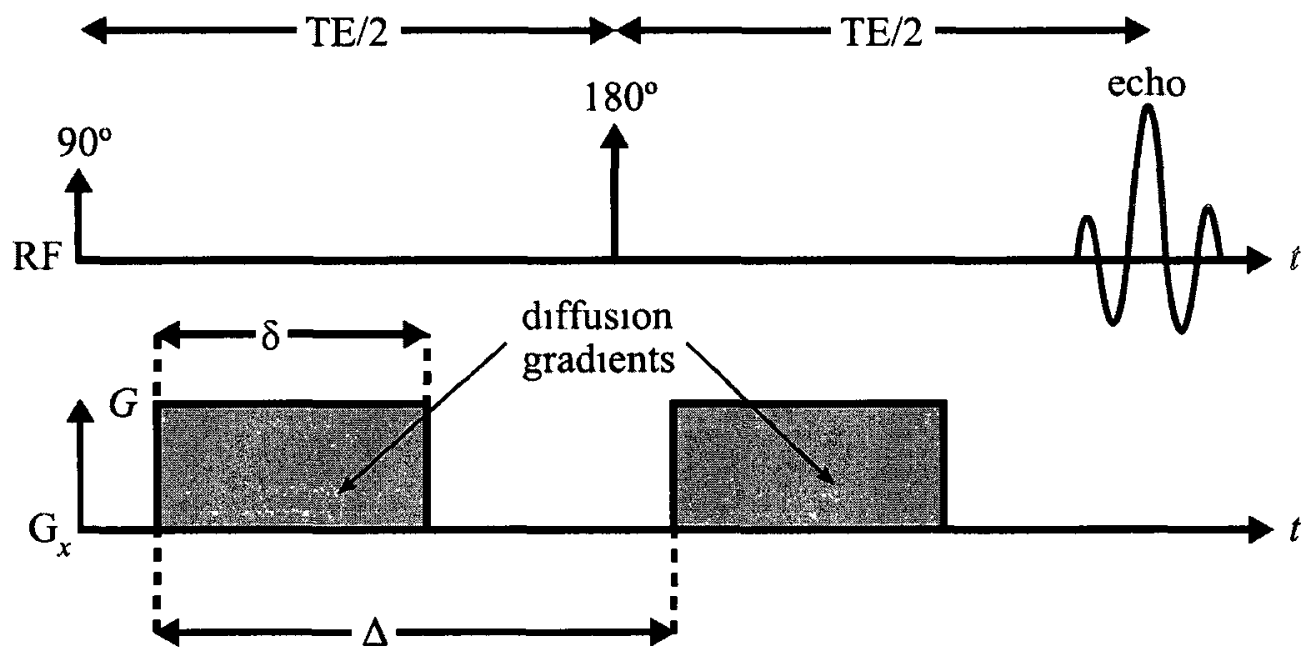

Fig. 2.6a. A schematic diagram of the Stejskal-Tanner Pulsed-Gradient Spın Echo (PGSE) sequence where $\delta$ is the pulse duration, $\Delta$ is the diffusion time, $G$ is the gradient pulse amplitude and TE is the time to the echo 


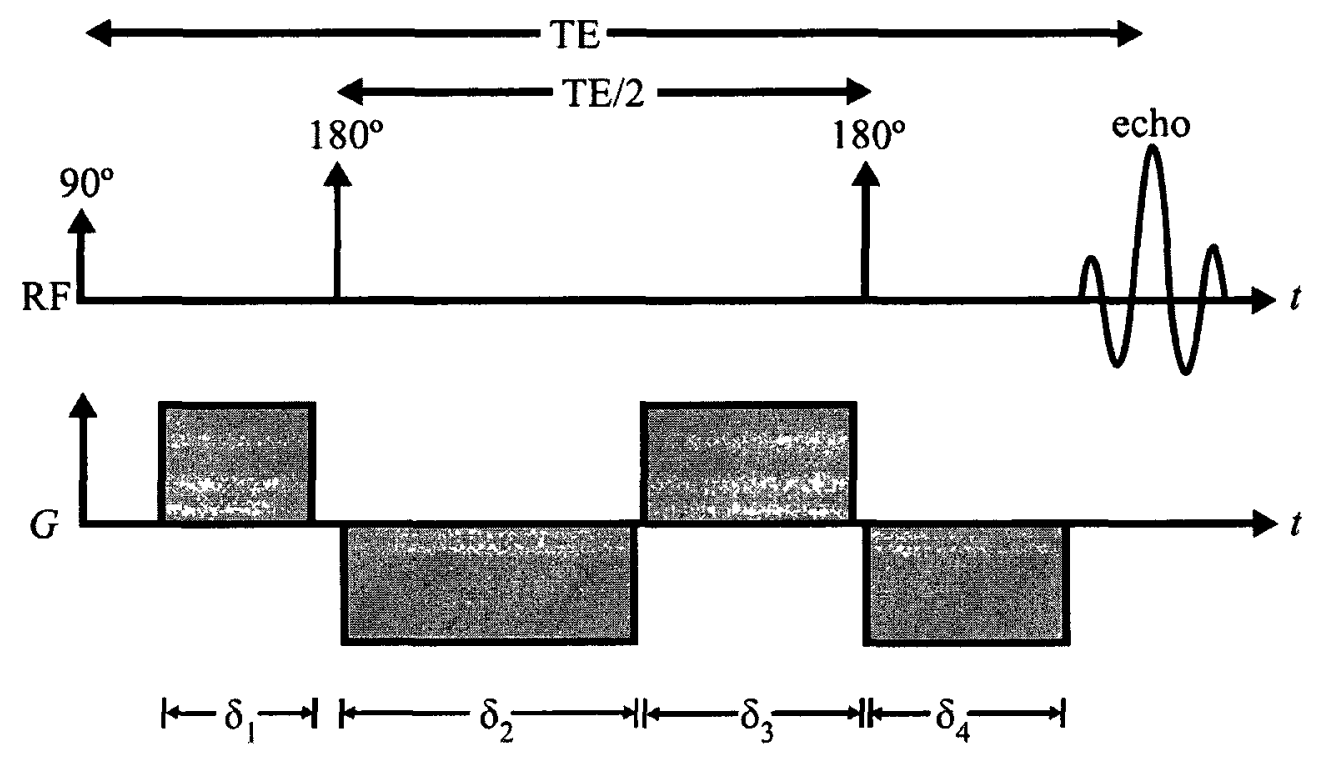

Fig. 2.6b. A schematic diagram of the twice-refocussed spin echo sequence [82] Gradient tımıngs are adjusted to minımıze the effect of magnetic fields generated by eddy currents Note that the timings are not drawn to scale

The first gradient pulse causes each spin to accumulate a phase shift that depends on the strength of the applied gradient and the spin's position along the axis of measurement, which will be considered to be the x-axis in this discussion [85] After the gradient is turned off, each spin will have accumulated a phase shift given by

$$
\varphi_{1}=\int_{0}^{\delta} \omega d t=\gamma \int_{0}^{\delta} G_{x} x d t=\gamma G_{x} x_{1} \delta
$$

where $\gamma$ is the gyromagnetic ratio, $G_{x}$ is the gradient amplitude and $x_{1}$ is the position of the spin along the $x$-axis at the time the gradient pulse is applied This phase shift will then be inverted by the $180^{\circ} \mathrm{RF}$ pulse such that $\varphi_{1}$ becomes $-\varphi_{1}$.

The second gradient pulse causes each spin to accumulate the phase shift 


$$
\varphi_{2}=\gamma \int_{\Delta}^{\delta+\Delta} G_{x} x d t=\gamma G_{x} x_{2} \delta
$$

where $x_{2}$ is the position of the spin along the $x$-axis at the time of the second gradient pulse The total phase accumulated by the spin, due to the pair of gradient pulses (and the $180^{\circ} \mathrm{RF}$ pulse), 1 s therefore

$$
\varphi=\varphi_{2}-\varphi_{1}=\gamma G_{x}\left(x_{2}-x_{1}\right) \delta
$$

which is proportional to the spin displacement along the $x$-axis that occurs between the two gradient pulses If durıng this time a spin remains stationary (1 e $x_{2}=x_{1}$ ), the echo amplitude will be unaffected because the net phase accumulation is zero Conversely, if during the diffusion time $t_{d}=\Delta$, the particles move in the $x$-direction, then the phase accumulation due to this displacement causes an attenuation of the MR signal The quantitative measurement of the spin displacement is made by comparison of the measured signal to a reference signal acquired without diffusion gradient pulses

It is not possible to measure the signal for a single spin, the total measured signal represents the net magnetization for all spins in the imaging voxel If the spins remain stationary throughout the experiment or if no diffusion gradients are used, the signal is given by

$$
S_{0}=K\left|\sum_{J=1}^{N} \mu_{J} \exp \left(l \varphi_{J}\right)\right|,
$$

where $K$ is a constant that incorporates various instrumental considerations (e $g$ amplifier gain), $\varphi_{J}$ is the phase of spin $J$ in the transverse plane, $\mu_{J}$ is its 
magnetic moment and the sum is over all spins in the voxel The net diffusion welghted signal therefore becomes (see Eq (2 31))

$$
S=K\left|\sum_{j=1}^{N} \boldsymbol{\mu}_{J} \exp \left(\imath \varphi_{J}\right) \exp \left(\imath \gamma \boldsymbol{G}\left(\boldsymbol{r}_{J}-\boldsymbol{r}_{0}\right) \delta\right)\right|
$$

where the vector $\boldsymbol{r}_{J}-\boldsymbol{r}_{0,}$ is the displacement of $\operatorname{spin}_{J}$

Alternatively, the signal at time $\Delta$ can be described in terms of the conditional probability function, $P\left(\boldsymbol{r}_{0} \mid \boldsymbol{r}, \Delta\right)$, introduced in the previous section, as [64]

$$
S(\Delta)=S_{0} \int_{-\infty-\infty}^{\infty} \int_{-\infty}^{\infty} \rho(\boldsymbol{r}) P\left(\boldsymbol{r}_{0} \mid \boldsymbol{r}, \Delta\right) \exp \left(\imath \gamma \boldsymbol{G}\left(\boldsymbol{r}-\boldsymbol{r}_{0}\right) \delta\right) d \boldsymbol{r} d \boldsymbol{r}_{0},
$$

where $S_{0}$ represents the initial signal with no diffusion gradients applied and $\rho(r)$ is the spin density This can also be expressed in $q$-space formalism as

$$
S(\Delta)=S_{0} \int_{-\infty}^{\infty} \int_{-\infty}^{\infty} \rho(\boldsymbol{r}) P\left(\boldsymbol{r}_{0} \mid \boldsymbol{r}, \Delta\right) \exp \left(\imath 2 \pi \boldsymbol{q}\left(\boldsymbol{r}-\boldsymbol{r}_{0}\right)\right) d \boldsymbol{r} d \boldsymbol{r}_{0},
$$

where $q \equiv \gamma G \delta / 2 \pi \quad$ For the case of free diffusion $P\left(r_{0} \mid r, \Delta\right)$ is Gaussian and the net signal loss due to the diffusing spins [64] is given by

$$
S(\Delta) / S_{0}=\exp \left(-4 \pi^{2} q^{2} D \Delta\right)=\exp \left[-(\gamma G \delta)^{2} D \Delta\right]
$$

In the discussion leading to Eq (2 36) it has been assumed that $\delta \ll \Delta$, however, this condition is seldom realized in practice Tanner [72] has shown that, for the PGSE sequence, the diffusion time, $t_{d}$, $(1 \mathrm{e}$ the time between tagging and detection during which the spins diffuse) is given by $t_{d}=\Delta-\delta / 3$, which clearly reduces to $t_{d} \approx \Delta$ when $\delta \ll \Delta$ To take into account the diffusion that 
occurs during a known finite pulse width, $\Delta$ in Eq (2 36) can be replaced by the effective diffusion time $\Delta-\delta / 3$ to give [72]

$$
S(\Delta)=S_{0} \exp \left[-(\not G \delta)^{2} D\left(\Delta-\frac{\delta}{3}\right)\right]
$$

Eq (2 36) can be simplified by introducing $b$ which is given by [72]

$$
b=(\not G \delta)^{2}\left(\Delta-\frac{\delta}{3}\right)
$$

In terms of $b$, the signal equation becomes

$$
S=S_{0} \exp [-b D]
$$

A more general expression for $b$ is derived in the next section

\subsubsection{Eddy Currents}

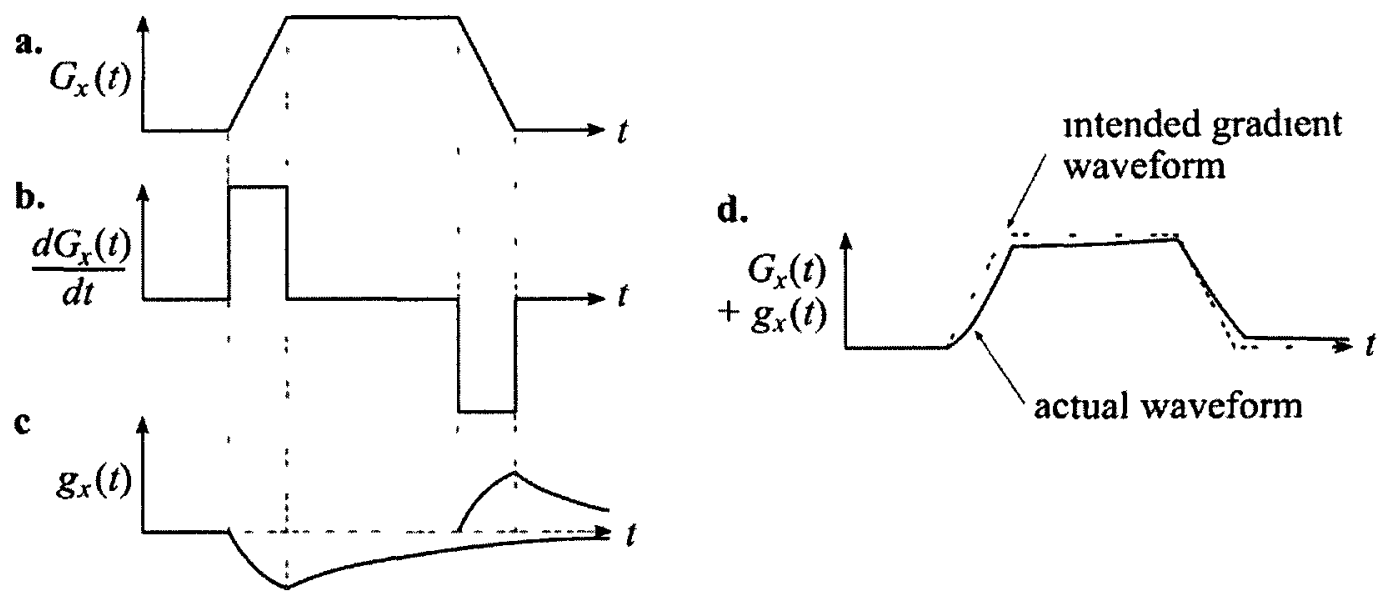

Fig. 2.7. (a) A gradient waveform, $G_{x}(t)$ (b) The first derivative of the gradient waveform, $d G_{x}(t) / d t \quad$ (c) One of the induced eddy current terms, $g_{x}(t)$ (d) The intended and the actual gradient waveforms [98] 
Due to the rapidly switching gradients in an MR sequence, the magnetic field in the scanner is constantly changing These changing magnetic fields induce electrical currents in conducting materials within the scanner The induced currents are called eddy currents, and result in magnetıc field gradıents whose direction is opposite to the change in field [82] (see also Figs $27 \mathrm{~b}$ and c) Eddy currents buld up during the ramp up and ramp down times of the gradients and decay during stationary phases The resulting waveform resembles a low-pass filter (Fig 27 d) $B_{0}$ eddy current gradients are spatially constant over the imaging volume

In our case, diffusion-weighting gradients are very large relatıve to most gradients used in other imaging sequences In combination with the EPI readout, which has a low bandwidth in the phase-encoding direction, DWI becomes easily susceptıble to eddy current artıfacts [82] Any translation or deformation of the Imaging volume can cause the voxel measurements to originate from different physical locations, which sometımes results in unusable images

By adding an additional refocusing RF pulse into the sequence and adjustıng the durations and amplitudes of the gradient pulses appropriately to mınımize eddy-current effects, Reese et al [82] were able to develop a sequence that substantially reduces image distortion caused eddy currents relative to the PGSE sequence 


\subsubsection{Equations governing the diffusive transport of magnetzation}

In the laboratory frame of reference Bloch's equation for the magnetization, $\boldsymbol{M}$, in a magnetic field $\boldsymbol{B}$, with an extra term added to account for 1sotropic diffusion is [73]

$$
\frac{d \boldsymbol{M}}{d t}=\gamma \boldsymbol{M} \times \boldsymbol{B}-\left(\frac{M_{x}}{T_{2}} \boldsymbol{x}+\frac{M_{y}}{T_{2}} \boldsymbol{y}\right)+\frac{\left(M_{0}-M_{z}\right)}{T_{1}} z+D \nabla^{2} \boldsymbol{M}
$$

This equation is often referred to as the Bloch-Torrey equation

For the Stejskal and Tanner experiment the spins will be affected by both the statıc field $\boldsymbol{B}_{0}$ and a linear magnetic field gradient $\boldsymbol{G}$ The resulting total field w1ll be

$$
B(r, t)=B_{0}+G \cdot r
$$

The Bloch-Torrey equation for the complex transverse magnetization $m$, defined as

$$
m(\boldsymbol{r}, t)=M_{x}(\boldsymbol{r}, t)+t M_{y}(\boldsymbol{r}, t),
$$

is given by

$$
\frac{d m}{d t}=-\imath \omega_{0} m-\frac{m}{T_{2}}-\imath \gamma \boldsymbol{G} \cdot \boldsymbol{r} m+D \nabla^{2} m,
$$

where $\gamma B_{0}=\omega_{0}$ The quantity $m(r, t)$ is not itself a directly observable quantity but rather it is a complex function constructed from the two experimentally determined values, $M_{x}(r, t)$ and $M_{y}(r, t)$ This complex function is very useful and is used frequently in MRI

The following substitution is used to formally elıminate the transverse relaxation and signal modulation [73] 


$$
m(\boldsymbol{r}, t)=\psi(\boldsymbol{r}, t) \exp \left(-\left(\imath \omega_{0}+\frac{1}{T_{2}}\right) t\right.
$$

resulting in

$$
\frac{d \psi}{d t}=-\imath \gamma \boldsymbol{r} \cdot \boldsymbol{G}(t) \psi+D \nabla^{2} \psi
$$

If the diffusion term is ignored for the moment, Eq (2 45) is easily solved to give

$$
\psi(\boldsymbol{r}, t)=C \exp (-\operatorname{tr} k(t))
$$

where $C$ is a constant of integration and

$$
k(t) \equiv \gamma \int_{0}^{t} G(t) d t
$$

At $t=0, C=\psi(\boldsymbol{r}, 0)=m(\boldsymbol{r}, 0)=M_{x}(\boldsymbol{r}, 0)+{ }_{1} M_{y}(\boldsymbol{r}, 0) \quad$ But since the reference phase in the rotating frame can be chosen arbitrarily, it can be assumed, without loss of generality, that $M_{y}(\boldsymbol{r}, 0)=0$ and $M_{x}(\boldsymbol{r}, 0)=M_{0}$, which means that $C=M_{0}$

If the diffusion term in Eq (2 45) is considered to cause an attenuation of the signal without affecting the phase, a trial solution can be generated from Eq (2 46) by replacing the constant $C=M_{0}$ with a time dependant function, $M(t)$, which is constructed as the product of $M_{0}$ and a real attenuation term By substitutıng this trial solution back into Eq (2 45) it can be shown that [73]

$$
\begin{aligned}
\frac{d M(t)}{d t} & =M(t) D \nabla^{2}[\exp (-\operatorname{lr} k(t))] \\
& =-M(t) D k(t) k(t)
\end{aligned}
$$

The solution to Eq $(248)$ at the echo time $t=T E$ is given by 


$$
\begin{aligned}
M(T E) & =M_{0} \exp \left[-D \int_{0}^{T E} \boldsymbol{k}(t) \boldsymbol{k}(t) d t\right] \\
& =M_{0} \exp \left[-D \gamma^{2} \int_{0}^{T E}\left[\int_{0}^{t} G\left(t^{\prime}\right) d t^{\prime \prime}\right)^{2} d t\right]
\end{aligned}
$$

To describe the sensitivity of MR sequences to diffusion, a very important quantity, $b$, the gradient factor, or $b$-value is defined as

$$
b=\gamma^{2} \int_{0}^{T E}\left(\int_{0}^{t} G(t) d t\right)^{2} d t^{\prime}
$$

By combinıng Eqs (2 50) and (2 51) the signal attenuation equation becomes

$$
S(T E)=S_{0} \exp [-b D],
$$

which is the same as Eq (2 39) derived in the previous section for the PGSE sequence in the limit where $\delta<<\Delta$ with $b$ given by Eq (2 38) If the diffusion weıghtıng involves a more complicated diffusion gradıent design, then $\mathrm{Eq}$ (2 51) can sometımes be difficult to evaluate analytically and numerical methods may have to be used It is important to emphasize that Eq (2 50) is strictly valıd only when diffusion takes place in a homogeneous, infinite and isotropic medium To be rigorous the signal attenuation has to be calculated differently when diffusion is restricted by impermeable barriers or if it is anisotropic

\subsubsection{Apparent Diffusion in Biological Tissues}

Discussion to this point has been limited to the case of isotropic free diffusion where water molecules can move equally freely in any direction The displacement probability in this case depends only on the temperature and 
mobility of the molecules and the signal attenuation caused by the diffusing spins 1s exponential

In biological tissue diffusion is complicated by various interactions and barriers to water movement, including organelle and cell membranes The water self-diffusion coefficient, $D$, may be the same in both cases but the measured diffusion coefficient is reduced in tissue The difference provides information about the cellular microstructure in the vicinity of the water molecules and its ability to hinder and/or restrict the movement of water To distınguish between these two situations, the term Apparent Diffusion Coefficient $(A D C)$ [74] is typically used to refer to the measured diffusion coefficient in tissues The $A D C$ describes the mobility of water molecules in tissue and this information can be used to develop insight into tissue microstructure A further implication of diffusion in tissue is that the displacement probability function can no longer be considered to be Gaussian The result is that the diffusion signal in biological tissue is not monoexponential, although, for the range of $b$-values used with DWI in most clinical applications, it is stıll often a reasonable approximation [75]

Diffusion in the majority of tissue types is reasonably isotropic and measuring the $A D C$ along a single direction is usually sufficient However, in highly organized tissues such as muscle and white matter, a scalar is no longer adequate to fully characterize the diffusion process Although some of the early research on diffusion-weighted MRI recognized differences in the $A D C$ along the $\mathrm{x}, \mathrm{y}$ and $\mathrm{z}$-axes in white matter, these three measurements do not provide enough information to completely describe the diffusion process Instead of a scalar 
diffusion coefficient, a diffusion tensor is required for these anisotropic cases and it becomes necessary to sample the $A D C$ along at least 6 different non-collinear directions to fully characterize this symmetric diffusion tensor

\subsubsection{Intravoxel Incoherent Motion Imaging}

The structure of the microvasculature of some organs in the body is such that, on the scale of a typical imaging voxel, the flow of blood through the microvasculature ( 1 e arterioles, capillaries and venules) can be viewed as being incoherent, pseudo-random motion A voxel typically contains several thousand capillaries which can be considered to be made up of multıple 'straight' segments oriented at many different angles [74] Blood flowing through the capillaries can thus be modeled as random, diffusion-like motion characterized by a pseudodiffusion coefficient, $D^{*}$ Blood flow in the microvasculature, viewed in this way, 1s sometimes referred to in the MR literature as IntraVoxel Incoherent Motion (IVIM) [74] The value of $D^{*}$ depends on the vascular geometry of the tissue as well as the rate at which blood flows through the microvasculature of the tissue being investigated and is generally at least an order of magnitude higher than the $A D C$ of the tissue water [74]

\subsubsection{Intravoxel Motion and MR Imaging}

Considering a population, $J$, of spins in the same voxel moving with an ınstantaneous velocity, $v_{J}(t)$, in a magnetic field gradient, $G(t)$, over a tıme interval 
$T$, one can define the dephasing, $\delta \phi_{j}$, for this group of spins as (see also Eq (2 29)) $[74,76]$

$$
\delta \phi_{J}=\gamma \int_{0}^{T} v_{j}(t) \boldsymbol{G}(t) t d t
$$

An expression for the complex transverse magnetization for the voxel can then be written quite generally in terms of the overall flow related dephasing, $\Phi$, and the amplitude, $F$, as

$$
F \exp (\imath \Phi)=F \sum_{J} p\left(\delta \phi_{\jmath}\right) \exp \left(\imath \delta \phi_{\jmath}\right),
$$

where $p\left(\delta \phi_{J}\right)$ is the distribution of the dephasings within the voxel of interest

In order to simplify $\mathrm{Eq}(254)$ it is necessary to introduce some assumptions about the geometry of the microvasculature of the tissue in the voxel and the motion of the spins in the vessels There are two different microcirculation models to be investigated Both consider the blood flow in the microvasculature to be microscopically incoherent

The first model [76] assumes that each $v_{j}(t)$ changes many times in the time interval $T$, such that the displacement for each group of spins in the voxel can be seen as a random walk process The motion is completely incoherent, in this case, and there is no net dephasing for the voxel but the signal amplitude will be attenuated This is very much analogous to the diffusion process discussed in section 231 If the motion is such that $p\left(\delta \varphi_{j}\right)$ is Gaussian then, as is the case with diffusion, the motion causes the magnitude of the transverse magnetization to be attenuated by the factor $F$ given by

$$
F=\exp \left(-b D^{*}\right)
$$


where $b$ is the gradient factor defined in section 2313 The pseudo-diffusion coefficient, $D^{*}$, associated with this process is analogous to the diffusion coefficient for the thermal random motion of the molecules and, it can be shown that (see also Eq (2 28)) [76]

$$
D^{*}=\frac{1}{6} \bar{l}_{m} \bar{v}_{m}=\frac{1}{6} \bar{l}_{m}^{2} / \bar{t}_{m},
$$

where $\bar{l}_{m}, \bar{v}_{m}$ and $\bar{t}_{m}$ are the average displacement, average speed and average duration, respectively, for the steps of the random walk process used in the model presented here to represent the motion of the spins in the microvasculature of the voxel $D^{*}$ has the same dimensions as a diffusion coefficient (e $\mathrm{g} \mu \mathrm{m}^{2} / \mathrm{ms}$ ) but is usually at least an order of magnitude larger than the $A D C$ for the tissue The similarities between diffusion and microvascular flow make it possible to use diffusion imaging methods to measure microvascular flow in the tissue as well

The second model of incoherent microcirculation [76] assumes that the diffusion time is short enough that the spins move with a constant speed and direction in a straight vascular segment during the measurement

Under these conditions Eq (2 53) becomes

$$
\delta \phi_{J}=v_{J} \cdot c=v_{J} c \cos \theta_{J},
$$

where

$$
c=\gamma \int_{0}^{T} G(t) t d t
$$

and $\theta_{J}$ is the angle between $\boldsymbol{v}_{J}$ and $\boldsymbol{c}$ (which is parallel to $\boldsymbol{G}(t)$ ) The overall effect of these de-phasings on the MR signal for the voxel can be computed as [76] 


$$
\left.F \exp (\iota \Phi)=\int_{0}^{\infty} \int_{0}^{2 \pi \pi} \int_{0} p(\theta) q(v) \exp (l c v \cos \theta)\right)^{2} \sin \theta d \theta d \varphi d v
$$

where $\theta$ and $v$ are independent variables and $p(\theta)$ and $q(v)$ are their respective distributions within the voxel $F$ and $\Phi$ are defined as the magnitude and phase of this voxel averaged function, which in general will be complex For the special case where the vascular segments are 1sotropically oriented Eq (2 59) simplifies to

$$
F \exp (\imath \Phi)=4 \pi \int_{0}^{\infty} q(v) \frac{\sin (c v)}{c v} v^{2} d v
$$

Note that, since the expression on the right hand side of this equation is real, the signal amplitude is attenuated but there is no overall de-phasing The order of magnitude of the attenuated signal can be estımated by performing numerical calculatıons usıng Eq (260), assuming $q(v)$ is a Gaussian distribution

When $c v$ is small, Eq (260) can be simplified further by using the Taylor expansion to first order for the sinc function to obtain [76]

$$
F \approx 1-\frac{1}{6} c^{2}\left\langle v^{2}\right\rangle
$$

where $\left\langle v^{2}\right\rangle$ is the variance of $q(v)$ defined by

$$
\left\langle v^{2}\right\rangle=4 \pi \int_{0}^{\infty} q(v) v^{2} d v
$$

Equatıng Eq (2 61) with

$$
F=\exp \left(-b \quad D^{*}\right) \approx 1-b \quad D^{*}
$$

gives

$$
D^{*} \equiv \frac{1}{6}\left\langle v^{2}\right\rangle\left(c^{2} / b\right)
$$


for the pseudo-diffusion coefficient for the second model

This model of incoherent microcirculation assumes only that the spins move with a constant speed and direction in straight microvascular segments during the measurement and that these segments are isotropically oriented Note that $D^{*}$, as given by Eq (2 64), not only depends on the dynamic properties of the microvascular network ( 1 e on $v$ ), but it also depends on sequence parameters in a complicated way through the factor $c^{2} / b$ Even so, the determination of $D^{*}$ can give very important insight into properties of the microvascular circulation

One important consideration when using IVIM is that the flowing component normally represents only a small fraction of the total volume of the voxel (e g in the brain) This fraction is expressed as $f$, the fractional volume of microvascular blood flowing in each voxel and it is equal to the ratio of $V_{d}$, the volume of water flowing in the microvascular compartment ( 1 e both inside blood cells and in the plasma) to the total volume of water in the voxel, $V_{\mathrm{H}_{2} \mathrm{O}}$

$$
f=V_{d} / V_{H_{2} \mathrm{O}}
$$

This quantity is called the active capillary fractional volume or the perfusion fraction

Inherent to the IVIM model are the following assumptions 1) there is one voxel compartment where the water molecules diffuse only, 2) there is a second compartment where the water molecules flow and diffuse inside the capillaries and 3) there is no (or at least very slow) exchange between these two compartments Under these conditions the following expression for the transverse magnetization in the voxel can be used 


$$
M_{x y}=(1-f) M\left(T_{1 d}, T_{2 d}\right) \exp (-b D)+f M\left(T_{1 c}, T_{2 c}\right) \exp \left(-b\left(D+D^{*}\right)\right),
$$

where the subscripts $d$ and $c$ stand for the diffusing only and the circulating components, respectıvely

The discussion of IVIM presented above suggests that DWI techniques can be made sensitive to blood flowing in the microvasculature of the tissue When this microcirculation is present, the attenuation of DW images is larger than would be expected from diffusion alone The effect of microvascular flow is observed as a pseudo-diffusion process and, in this case, the decay is b1exponential instead of mono-exponential With the IVIM approach the b1exponential nature of the decay curve is recognized This allows for the determination of $D, D^{*}$ and $f$, three important fundamental tissue parameters These IVIM parameters can give more information about the tissue than can the $A D C$, which is effectively just an average decay constant for the measured diffusion decay which is inherently assumed to be exponential even though this is seldom the case in tissues The $A D C$, therefore, has no direct intrinsic physical meaning The $A D C$ values obtained clinically often depend on the $b$-values used and etther over or underestimate the true diffusion coefficient for water, depending on which part of the diffusion decay that is sampled

In order to precisely determine the IVIM parameters, acquisitions with many $b$-values are necessary The higher range of $b$-values is related exclusively to diffusion as the signal from the circulating spins has decayed to zero for this part of the decay due to the large amplitude gradients employed and the relatıvely large displacements involved The lower range of $b$-values corresponds to smaller 
amplitude gradients and the acquired images for these $b$-values contain contributions from both diffusion and the microcirculation

Difficulties may arise when IVIM measurements are performed on tissues, such as the brain, where $f$ is small In this case the diffusion decay must be measured very carefully in order for the regression analysis to be able to reliably determine all three IVIM parameters Sometimes, when this is the case, a region of interest (ROI) based analysis is preferable to a pixel-by-pixel approach This, however, is not a problem for either the placenta or the liver studies presented in this thesis since $f$ is relatively large for both of these tissues

The IVIM model considers the water in the system to be in two distinct compartments, these being the intravascular and extravascular compartments It also assumes that the water molecules stay in the same compartment for the full duration of the experiment In this case, the measured diffusion decay will be b1exponential with the decay constants corresponding to the intrinsic diffusion coefficients of these two compartments This is known as the slow exchange limit

However, if the water molecules in fact can move freely between these two compartments and this exchange is very fast on the time scale of the experiment, the spins, on average, will spend an equal amount of tıme in each of these environments In this case, the system decays monoexponentially with a rate that represents the weighted average of the intrinsic intravascular and extravascular rates This is known as the fast exchange limit

When exchange exists but it cannot be considered either fast or slow for the experiment being considered, this is referred to as intermediate exchange The 
diffusion decay for this case is much more complicated If this decay is fit to a b1exponential decay (as is often the case), a quality fit to the data can frequently be found but the two decay constants obtained, in this intermediate exchange case, do not correspond to the intrinsic diffusion coefficients for the two compartments

\subsection{Motion Tracking with Prospective Acquisition Correction (PACE)}

For most MRI applications, motion can adversely affect image quality Some types of motion effects can be corrected for in post processing but ideally the acquisition of data in real time, with good image quality, in spite of motion of (or within) the subject, is preferable The latest strategy offered on Siemens MR scanners to deal with motion are the inline techniques called 1D-PACE, 2DPACE, and 3D-PACE 1D-PACE is ideal for cardiac imaging because it is very fast $(30 \mathrm{~ms})$ 2D-PACE is commonly used for abdominal imaging when breathing motion can be problematic The small flip-angles used in 2D-PACE leave the magnetization in the imaging volume of interest practically undisturbed 3D-PACE can detect and correct, linear translational and/or rotatıonal rigid body motion in 6 degrees of freedom in real time and is used primarily for motion correction in neurological studies

In 2D-PACE, the technique used for most of the data acquisition presented in this thesis, a navigator echo module precedes the imaging sequence $[77,78]$ This module acquires a navigator echo MR signal from a small area defined by an ROI placed on a localizer ımage by the scanner operator over the object whose motion is to be tracked (see blue box in Fig 2 8) From this 


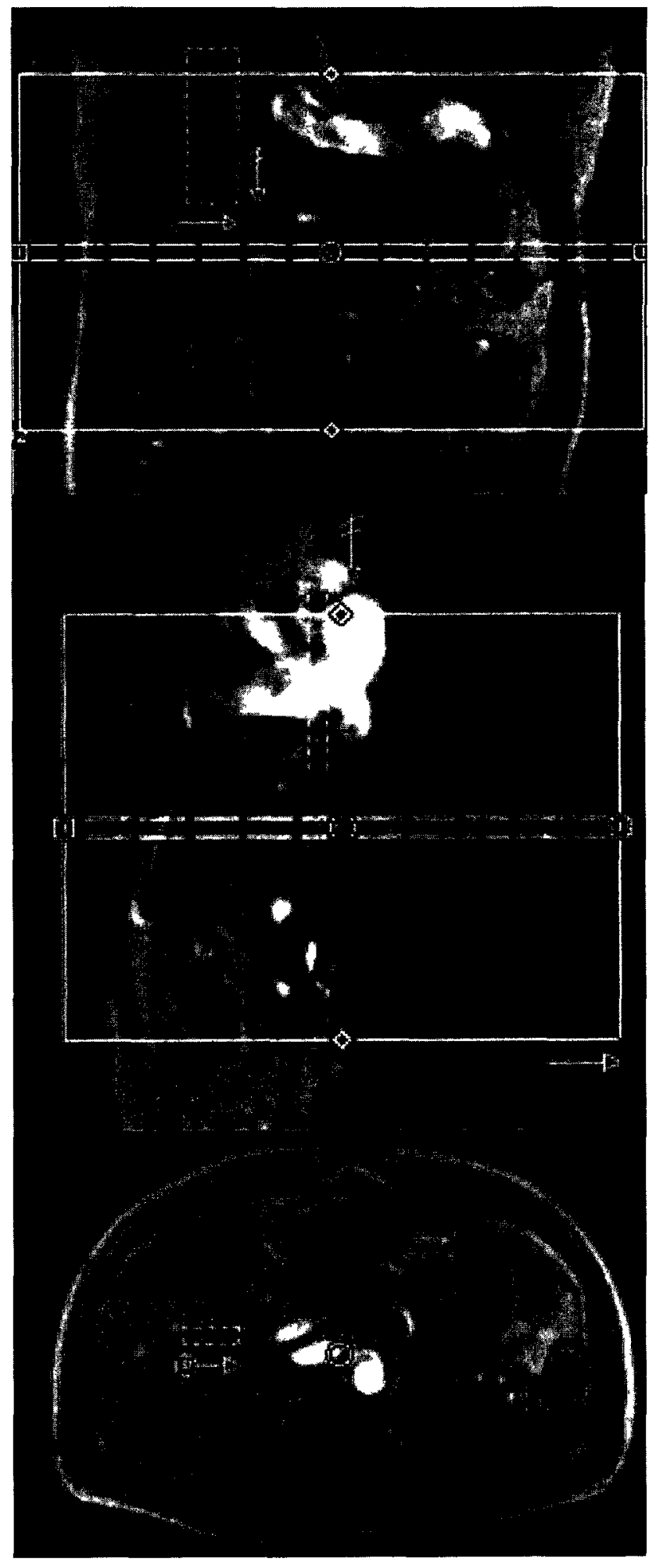

Fig. 2.8. The figure contains a screen capture from the MR scanner showing the location of the navigator echo ROI ( $1 \mathrm{e}$ the blue box) positioned over the diaphragm for the 2D-PACE navigator echo respiratory triggering technique The yellow box indicates the volume that is to be imaged 
signal a 1D projection along the length of the ROI ( $1 \mathrm{e}$ the blue box) is generated Normally, for abdominal imaging, the tracked object is the diaphragm since the weak signal from the lung above and the relatively strong signal from the liver below provide good contrast for tracking the motion of this interface

The first step in performing image acquisition with 2D-PACE is a trainıng period where the breathing cycle is recorded (F1g 29) No image acquisition is done during this time The position of the diaphragm is determined by the change in signal intensity along the axis of the ROI The interface contrast needed between the diaphragm and the lung has to be high in order to allow easy automatic detection

Once the triggering algorithm has the necessary information, image acquisition begins $1 \mathrm{D}$ projections through the diaphragm are continuously acquired and sent to a respiratory triggerıng algorithm When this routine determines that the diaphragm is in the desired location, the imaging sequence acquires the desired number of slices (see Fig 210 ) However, the Image data are only accepted if the position of the diaphragm is found to have been within the acceptance window (set to be $\pm 2 \mathrm{~mm}$ in our protocols) when the data were acquired The sequence design and the trigger algorithm allow one trigger event per respiratory cycle, with the image acquisition occurring during the end expiration phase

A low resolution gradient echo sequence (FLASH) with a flip angle of $3^{\circ}$ is used in the 2D-PACE navigator echo module The small flip angle ensures that 


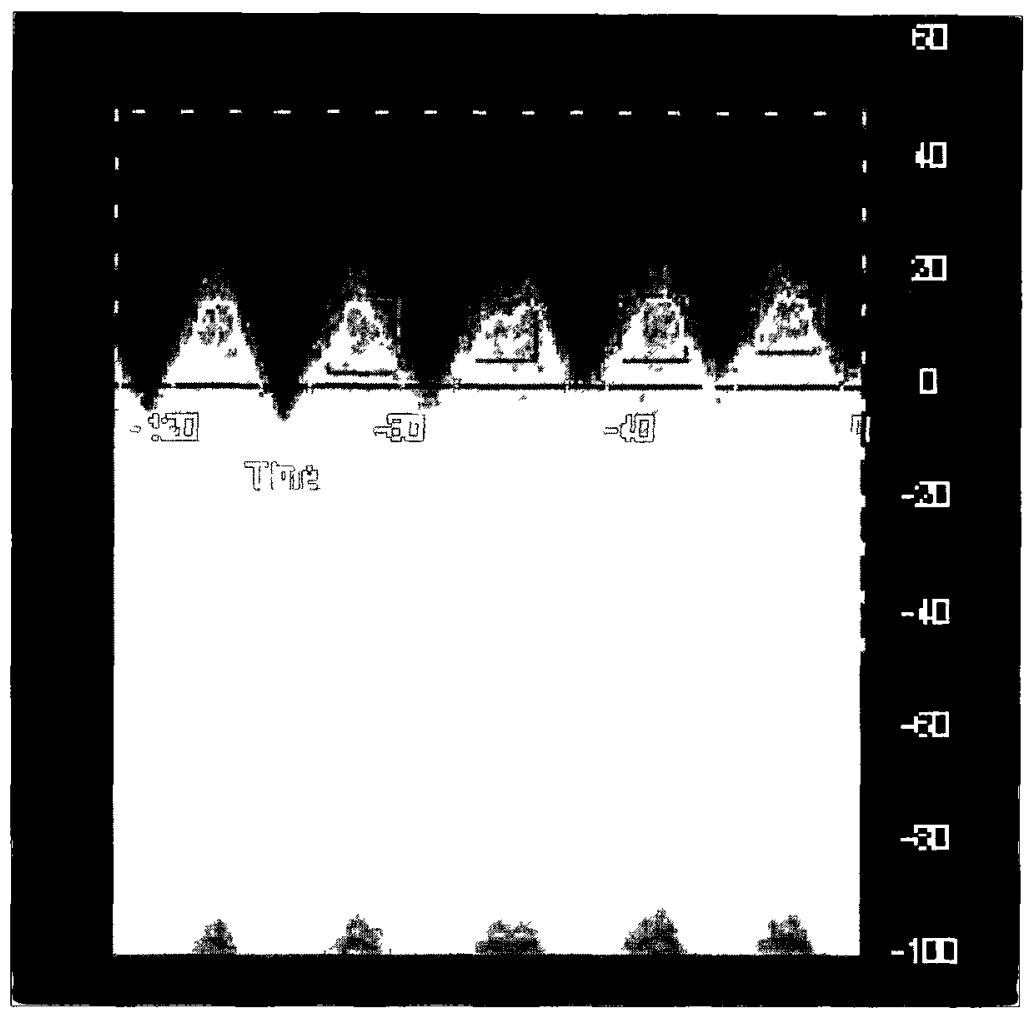

ThIg. 2.9. The motion of the dnaphragm durng free breathing IS Iepresented by the green lune un this screen capture from the MR scanner The red boxes depict the acceptance windows that the respiratory algorithm has set for the tranming period

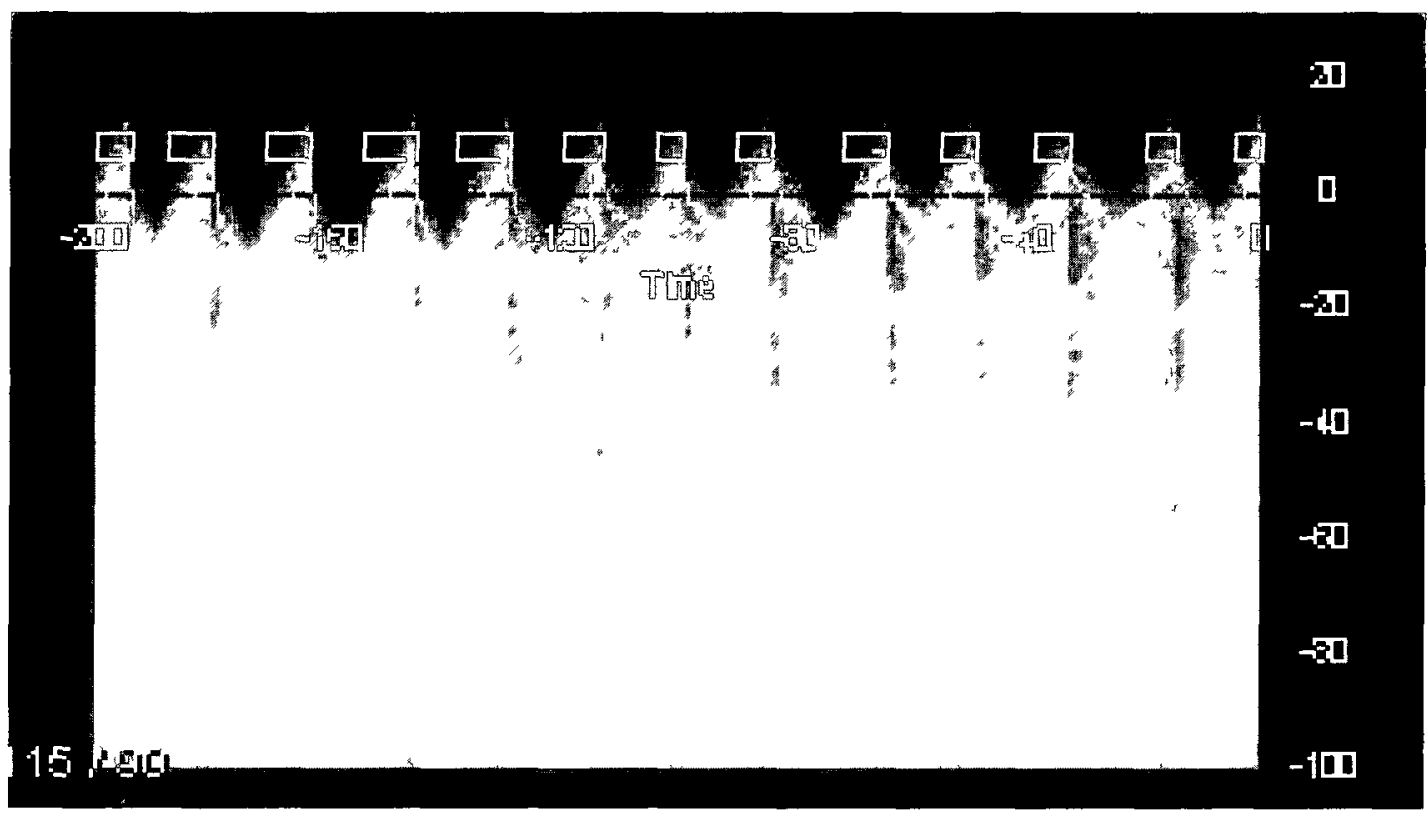

Fig. 2.10. The green line represents the motion of the diaphragm during free breathing in this screen capture from the MR scanner The yellow boxes represent the acceptance windows for mage acquisition at the end of exprration 
no magnetization saturation of the tissue of interest occurs (1 e no saturation stripes from the navigator echo module can be seen in the anatomical images) The time needed to acquire a navigator echo for $2 \mathrm{D}-\mathrm{PACE}$ is around $100 \mathrm{~ms}$

The 2D-PACE method, which is unique to Siemens, is very robust and is makıng free-breathıng abdomınal MR ımagıng with very good ımage quality a clinical reality The advantages of PACE can be exploited in numerous ways This technique has greater accuracy than any other form of respiratory gating and no additional hardware is necessary for the imaging protocol

\subsection{Human Placenta}

The placenta $[79,93,94]$ a unique organ that connects the fetus to the uterine wall, plays a vital role in the nutritive, respiratory, and excretory functions of the fetus The placenta is the one organ that supplies the fetus with oxygen and nutrients and allows fetal residues to be disposed of through the maternal kidneys

The mature placenta is about $22 \mathrm{~cm}$ long and its thickness, which varies from one subject to another, is usually greatest at the center and smaller perıpherally The color is dark reddish-blue or maroon since it contains a lot of blood from both the fetal and maternal hemodynamic systems Its weight varies as well but, on average, it weighs about $500 \mathrm{~g}$

The umbilical cord, another very important component of the fetomaternal system is approximately $55-60 \mathrm{~cm}$ in length and contains two arteries and one vein The umbilical cord connects the placenta to the fetus The two arteries in the umbilical cord radiate in opposite directions from the attachment of 
the cord on the inner surface of the placenta and divide into smaller arteries that go into the depths of the placenta through hundreds of branching and interlacing strands of tissue known as vill Although there is only one vein in the umbilical cord, it divides into two branches at the surface of the placenta and these branches, like the umbilical arteries, lead to a network of vessels and microvessels that permeates the placenta

\subsubsection{Placenta Development}

The development of a new human being begins at fertilization when a sperm cell fuses with an ovum During the first five days after conception a structure called a blastocyst, which is made up of 70-100 cells, is formed The blastocyst consists of 1) an aggregation of cells known as the embryoblast that eventually forms the embryo, 2) a fluid-filled cavity and 3) an outer layer of cells known as the trophoblast which surrounds the embryoblast and the fluid-filled cavity It is the trophoblast that eventually develops into the placenta As the pregnancy evolves, another layer of cells called the mesoderm forms on the inside surface of the trophoblast The trophoblast and the mesoderm together form the chorion

The development of the placenta begins upon implantation of the blastocyst to the uterine wall, the endometrium, and it contınues to grow throughout the pregnancy The outer layer of the placenta forms from the trophoblast which is the outer layer of the blastocyst This trophoblastic layer of the chorion develops numerous tree-like structures, called chorionic villi, on all 
parts of its surface At the site of implantation, the villi invade and destroy the decidua, a layer of cells lining the uterus, and eventually deeper layers of the endometrium as well This trophoblastic invasion also disrupts the spiral arteries of the decidua causing blood to flow into the intervillous space of what will become the placenta Development of the maternal blood supply to the placenta is thought to be complete by the end of the first trimester of pregnancy which is approximately 12-13 weeks after conception Vill associated with parts of the decidua away from the implantation site degenerate and leave behind smooth chorion However, the vill at the decidua basalis, that part of the decidua located at the implantation site, increase in number and size By week 18 of the pregnancy the decidua basalis represents $15-30 \%$ of the whole decidua By the end of the pregnancy over 1 billion microvillı per square centımeter will have formed Geometrically, the vill 1 are very complex The branches of the vill can be considered to contain many short randomly oriented capillary sections and blood motion in the villı can be viewed as a random walk process By the end of the fourth month most, but not all, cells of the decidua basalis have been destroyed, however, the trophoblast continues to invade and erode deeper layers of the endometrium to enlarge the intervillous space The remaining decidual tıssue forms wedge-shaped areas called placental septa that subdivide the placenta into lobules called cotyledons The mature human placenta consists of $15-20$ of these cotyledons with each one containing numerous vill and their many branches Because septa don't reach the chorionic plate, the fetal side of the 
placenta from which the vill originate, the maternal blood in the intervillous spaces can flow between cotyledons

The growth of the placenta parallels fetal growth and, as a result, an approximately constant perfusion fraction is expected throughout the pregnancy In the third trimester of pregnancy the volume of a single cotyleton, including both the villous and the intervillous space, would typically be about $1 \times 1 \times 2 \mathrm{~cm}^{3}$ The fractional volume of intervillous space relative to the total villous plus intervillous volume is approximately 05

\subsubsection{Blood Circulation in the Placenta}

Both fetal and maternal blood flow in the placenta at the same time The fetal blood passes through blood vessels of placental villı and maternal blood moves through the intervillous spaces Maternal and fetal blood are separated from each other in the placenta by the villı walls so they do not mix

There are about 80 to 100 places in the decidua basalis where maternal blood spurts from the spiral arteries into the intervillous space The maternal blood enters the intervillous space with relatively high pressure and creates a relatively high velocity flow at this entry location The blood then continues to flow around the villı in the intervillous spaces at a much lower speed, bathing the vill in nutrient rich maternal blood that eventually returns, via the endometral veins, to the maternal circulation There is a drop in pressure between the maternal blood entering the intervillous spaces and the blood returning to the venous network which induces the crrculation of maternal blood around the vill 
There are about $150 \mathrm{ml}$ of maternal blood in the intervillous spaces, which is exchanged 3 or 4 times a minute Maternal blood flow is approximately $600-700$ $\mathrm{ml} / \mathrm{min}$ at term

The fetal blood flows through the microvasculature of the v1ll 1 The smallest of these microvessels are capillaries which are only microns in diameter The fetal blood absorbs oxygen and nutrients from the maternal blood through the walls of the villi, and gives up its waste products in the same manner This purified, nutrient-enriched blood is carried to the umbilical cord by a network of fetal veins in the placenta, which unite on the surface of the placenta, at the attachment of the cord to form the umbilical vein In a similar fashion, two umbilical arteries take waste products such as carbon dioxide from the fetus back to the placenta where they are picked up by the maternal blood, the circuit to and from the placenta being thus complete

Geometrically, the vill 1 are very complex The branches of the villı are composed of very many short randomly oriented microvascular segments Consequently, the blood motion in the villi can be viewed as a random walk This highly vascular structure of the placenta allows the assessment of perfusion in the placenta by use of the IVIM model

\subsection{Human Liver}

The liver $[79,94]$ is the largest glandular organ in the body and has many metabolic and secretory functions It is located in the superior part of the abdominal cavity beneath the diaphragm Its largest transverse measurement is 
from 200 to $225 \mathrm{~cm}$ and weighs about 12 to $18 \mathrm{~kg}$ for an adult liver, depending on the overall body size Its consistency is that of a soft solid It is easily lacerated and it has a dark reddısh brown color due to its high vascularity

\subsubsection{Structural Anatomy of the Liver}

Anatomically, the liver consists of the right, left, caudate, and quadrate lobes The right lobe accounts for one half to two thirds of the total liver volume The right and left lobes have equal functionality, even though their sizes may differ significantly These lobes are divided by a line extending from the inferior vena cava superiorly to the middle of the gallbladder fossa inferiorly Anteriorly, the right lobe is separated from the smaller left lobe by the falciform ligament Inferiorly, the caudate lobe is positioned near the inferior vena cava, and the quadrate lobe is adjacent to the gallbladder The falciform ligament attaches the liver anteriorly to the abdominal wall and superiorly to the diaphragm The ligamentum teres extends from the falciform ligament to the umbilicus

\subsubsection{Functional Anatomy of the Liver}

The liver secretes up to $1,000 \mathrm{ml}$ of bile, a fluid necessary for digestion, every day A duct system carries bile to the common hepatic duct which connects with both the duodenum of the small intestıne, via the common bile duct, and with the gallbladder, via the cystic duct, where it is concentrated and stored The presence of fat in the duodenum stımulates the flow of bile out of the gallbladder into the small intestıne 
Blood is carried to the liver via two large vessels called the hepatic artery and the hepatic portal vein The hepatic artery carnies blood containing oxygen from the aorta The hepatic portal vein carries blood containing nutrients and xenobiotics extracted from digested food in the small intestine These blood vessels subdivide in the liver in a tree-like manner, terminating in very small capillaries Each capillary leads to a lobule Liver tissue contains thousands of lobules made of hepatic cells called hepatocytes, the basic metabolic unit of the liver Microscopically, each lobule consists of a group of hepatocytes that are approximately polyhedral in shape They vary in diameter from 12 to $25 \mu \mathrm{m}$ and contain one, or sometımes two, distinct nucle1 The hepatic cells are arranged in irregular radiatıng columns between which the blood channels, called sinusoids, can be found The blood goes through the sinusoids, from the circumference to the center of the lobule, and ends up in the intralobular vein that goes through the center of the lobule The lobular base is connected to the sublobular vein into which the intralobular vein opens directly The sublobular veins unite to form larger and larger trunks which end finally in the hepatic veins There are three large hepatic trunks that unite and open into the inferior vena cava

Between the hepatocytes there are minute channels called bile canaliculı These passages are little spaces left between the contiguous surfaces of the cells, and they are always separated from the blood capillaries by at least half the width of a liver cell The bile, which is secreted by the hepatocytes, is collected in these bile canaliculi Following the same model as for the blood vessels, the biliary ducts form two main trunks, leave the liver at the transverse fissure and their 
union forms the common hepatic duct The lobules of the liver contain all of the essential components of a secreting gland cells where the secretion is formed, blood vessels in close proximity to the secreting cells and ducts that collect the secretion and carry it away

Hepatocytes represent about $60 \%$ of the tissue in the liver and perform more metabolic functions than any other cells in the body The Kupffer cells, also present in the lobule, line the smallest vascular channels and have an important role in blood formation, antibody production and the breakdown and disposal of cellular debris The liver secretes bile, it stores vitamins, glycogen and other substances, it synthesizes blood-clotting factors, it removes toxins and waste from the blood and it also regulates blood volume and destroys old red blood cells

The highly vascular structure of the liver, as presented above, allows IVIM to be used to assess perfusion in the liver 


\title{
Chapter 3
}

\section{Using an IVIM Model to Assess the}

\author{
Liver Microvasculature
}




\subsection{Introduction}

In this chapter, in vivo diffusion weighted (DW) MR measurements on human liver are presented which are sensitive to microscopic motion in biological tissues, including the self diffusion of water molecules and microcirculation of blood in the capillary network of the tissue

Motion related artifacts have limited the usefulness of diffusion weighted 1maging (DWI) of abdominal organs and for the evaluation of abdominal pathology in the past Since DW sequences are designed to be very sensitive to motion, it is challenging to perform artıfact-free DWI in the presence of bulk motion due to respiration, peristalsis and blood flow Nevertheless, a few recent clinical studies have shown that DWI can be successfully used for the diagnosis of liver fibrosis and cirrhosis [9], the detection and characterization of liver lesions [10-28], and the assessment of liver tumor treatment response [34-41] Most of these studies have used the mono-exponential apparent diffusion coefficient (ADC) model, even though the decay is clearly bi-exponential Unfortunately, the reported ADC values vary over a very wide range (300\% variation) for both normal liver and focal hepatic lesions [42] There is thus a need for the implementation and validation of more reliable data acquisition techniques and ımage analysıs tools for DWI in the abdomen and, in partıcular in the liver, the organ that is the subject of this chapter

It is overly simplistic to assume that the attenuation of the diffusion signal intensity as the $b$-value increases is a simple exponential decay in tissue, partıcularly in highly vascular tissues such as the liver Molecular motion causes 
a reduction in the signal intensity which is proportional to the displacement of the signal inducing molecules during the measurement The signal initially (1 e at low $b$-values) decays quickly, due to the microcirculation, followed by a slower attenuation, corresponding to diffusion, as the $b$-value increases The b1exponential Intra Voxel Incoherent Motion (IVIM) model is a more realistic approach for vascular tissues and it is the model that will be used in this study to take into account the part of the decay that is sensitive to blood motion in the microvasculature

To our knowledge there are only two reported IVIM studies of the liver One of these used a breath hold $(\mathrm{BH})$ protocol [12] The other employed a free breathıng (FB) protocol using a respiratory triggered (RT) sequence where a pneumatic belt was used to generate the trigger pulse [9] There are no reports of IVIM studies of the liver that used a navigator echo respiratory triggered sequence such as the one used for the work presented in this chapter

Parallel ımagıng [44,45] and respıratory triggerıng [46] have been proposed to improve the image quality and precision of diffusion measurements in the abdomen Parallel ımaging is a relatıvely new technique for MR signal acquisition that takes advantage of the multiple coil elements in state-of-the-art phased array rf receiver coils This technology can be used to reduce the acquisition time or increase the number of measurements performed in the same time or a combination of the two The use of parallel imaging in combination with modern phased array coils leads to a considerable improvement in the quality of the data 
Respiratory triggering synchronizes image acquisition with the patıent's breathing cycle in order to avold motion artıfacts and to ensure that the anatomy of interest is at the same location within the FOV for each repetition of the sequence The use of respiratory triggering allows the acquisition time to be as long as required ( $1 \mathrm{e}$ it is not limited to a single breath hold) and removes the patient discomfort associated with breath holdıng Respiratory triggerıng was initially implemented in MRI using respiratory bellows or pneumatic belts which transmit a trigger pulse to the scanner to start image acquisition at the appropriate part of the respiratory cycle and to ensure that, for mult1-acquisition protocols, the signals are all acquired for the same part of the respiratory cycle These methods have largely been replaced by techniques that use navigator echoes to monitor the respiratory cycle by trackıng the motion of the diaphragm

On the Siemens scanner used for the research reported here, Navigator echo Respiratory Triggerıng (NRT) is referred to as Prospective Acquisition Correction or 2D-PACE This technique requires no additional hardware and patient set up is much easier Furthermore, using NRT sequences ensures that the measurement is done with the abdominal organs in the same location for each respiratory cycle With 2D-PACE, a navigator echo, which is acquired from a small region of interest (ROI) placed perpendicular to the diaphragm, allows the position of the diaphragm to be accurately tracked The measured diaphragm positions are then passed to a respiratory triggerıng algorithm Image acquisition is triggered when the diaphragm is withın $\pm 2 \mathrm{~mm}$ of a prescribed location Image 
acquisition is done at the end of expiration and there is one trigger event per breathıng cycle For more detals about 2D-PACE see Sectıon 24

The primary aims of the work reported in this chapter were to improve the methodology used for measuring DW images in the liver and to determine the true values for the IVIM parameters (1 e the diffusion and pseudo-diffusion coefficients and the perfusion fraction) for normal liver tissue in vivo $\mathrm{BH}$ and FB protocols were investigated and the obtained IVIM parameters are compared

For the BH-protocol it was only possible to report on 3 subjects, even though 10 subjects were scanned with this protocol, because of strong chemical shift and/or motion artifacts on many of the images In spite of the small number of subjects, the results for the BH-protocol were included here for the sake of comparison with the results obtained with the FB-protocol Although the FBprotocol is far superior, as the results in this chapter clearly show, one potential advantage of the BH-protocol used here is that the initial part of the b1exponentıal diffusion decay is better defined since more small $b$-values were used Whether or not this is a true advantage will be considered

\subsection{Materials and Methods}

\subsubsection{Subject Population}

This study was approved by the Ottawa Hospital Research Ethıcs Board Written informed consent was obtained from all participants Eighteen volunteers (8 women and $10 \mathrm{men}$ ), ranging in age from 17 to 61 years old, were recruited The analysis presented in this chapter is for 11 volunteers The first 3 of these subjects 
were investigated with a BH-protocol while for the next 8 a FB-protocol, which included a NRT 2D-PACE DW sequence, was used See Sections 24 for a more detalled discussion of 2D-PACE Images for the other 7 volunteers, all acquired with the BH-protocol, could not be considered for analysis as they were affected either by artifacts or by sudden involuntarily motion of the subject None of the subjects were patients at the hospital, all were volunteers in apparent good health At the tıme of imaging, all volunteers were considered to have a normal liver

\subsubsection{MR Technique}

MR imaging was performed at the General Campus of The Ottawa Hospital using a 15 Tesla (T) Siemens Magnetom Symphony TIM® (Siemens Medical Solutions, Erlangen, Germany) MR system with a maximum gradient strength of $30 \mathrm{mT} / \mathrm{m}$ The first group of subjects were imaged with the BH-protocol on the Siemens Magnetom Symphony Quantum ${ }^{\circledR}$ - Maestro Class system using the torso phased array coil placed anteriorly and elements of the spine phased array coil posteriorly The second group of subjects were imaged after a significant upgrade to the Siemens scanner which took it from a Maestro Class system to a TIM system For this second group, the FB-protocol was used Signal acquisition was done with the torso phased array matrix coil anteriorly and elements of the spine phased array matrix coll posteriorly For all volunteers, the full protocol consisted of The Ottawa Hospital's routine clinical liver protocol with additional DWI sequences added An example of an image of a typical healthy liver can be seen in Fig 31 


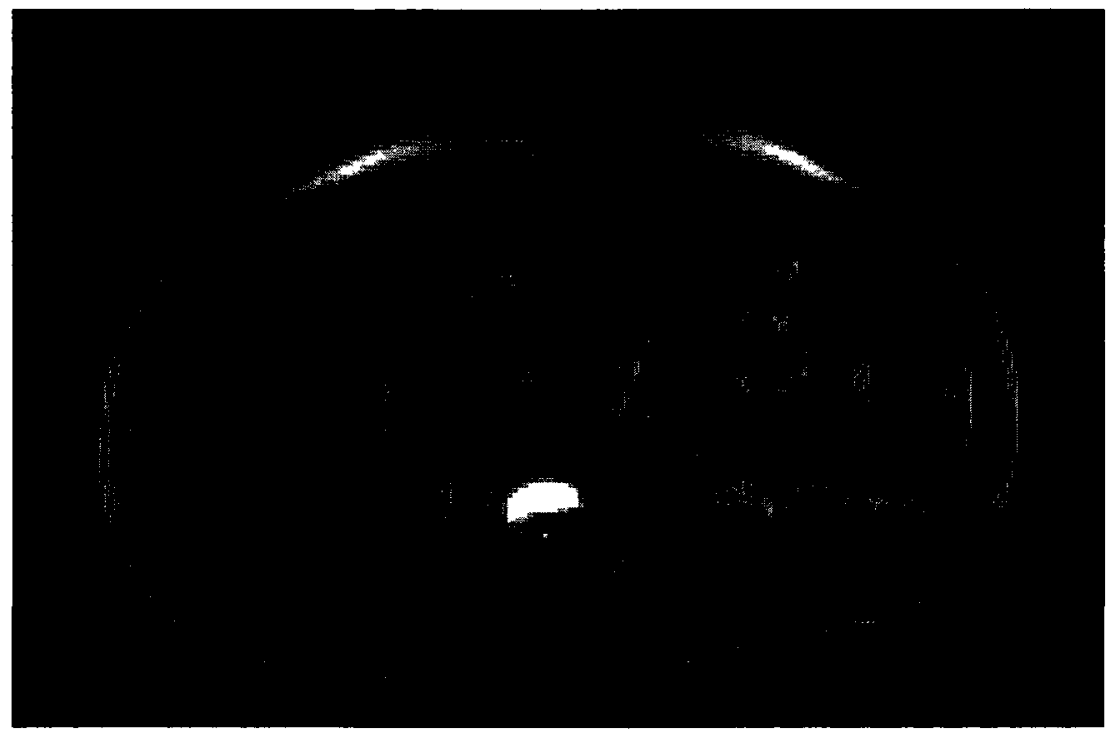

Fig. 3.1. An image of a healthy liver (slice 11, volunteer 16) A HASTE sequence was used for this acquisition

\subsubsection{Breath hold protocol}

DW 1mages for all $b$-values and all slices were acquired during a single 30 to $35 \mathrm{~s}$ breath hold using a DW single-shot echo-planar-1maging (DW SS EPI) sequence called ep2d_diff, with the following parameter settıngs $\mathrm{TR}=2500 \mathrm{~ms}, \mathrm{TE}=91$ $\mathrm{ms}, 4$ to 6 axial $10 \mathrm{~mm}$ thick slices were acquired with an interslice gap of 25 $\mathrm{mm}, \mathrm{NEX}=1, \mathrm{FOV}=380 \mathrm{~mm}$, phase $\mathrm{FOV}=75 \%$, matrix size $=128 \times 96$ and the voxel volume was $3 \times 3 \times 10 \mathrm{~mm}^{3}=90 \mathrm{~mm}^{3}$ The total imagıng time for these DW sequences was 30 to $35 \mathrm{~s}$, depending on the number of slices acquired Spectral fat saturation was used to suppress chemical shift artıfacts For each slice DW Images were obtained for the following $10 b$-values $0,10,20,30,50,90,150$, 300,500 and $800 \mathrm{~s} / \mathrm{mm}^{2}$ The diffusion gradients were applied in the slice selection direction (head-to-foot) in all cases With this protocol the number of 
slices that could be acquired was limited by the subject's ability to hold his or her breath resultıng in incomplete liver coverage

The DW SS EPI sequence provided by Siemens with the scanner is restricted to $b$-value increments of $50 \mathrm{~s} / \mathrm{mm}^{2}$ To make the sequence more appropriate for IVIM measurements, the sequence was modified to allow for smaller $b$-values by changing the increment from $50 \mathrm{~s} / \mathrm{mm}^{2}$ to $10 \mathrm{~s} / \mathrm{mm}^{2}$ This was done using source code and the IDEA sequence programming environment, both provided by Siemens under a research agreement

A water phantom at room temperature was used to validate the IVIM DW imaging sequence used with the BH-protocol A monoexponential signal decay with a decay constant of $D=200 \pm 006 \mu \mathrm{m}^{2} / \mathrm{ms}$ was obtained This result is in very good agreement with the reference diffusion coefficient for water of $D=20$ $\mu \mathrm{m}^{2} / \mathrm{ms}$ at $22^{\circ} \mathrm{C}[80]$

\subsubsection{Navigator echo respiratory triggered free breathing protocol}

With the FB-protocol it was possible to image the entıre liver DW images were acquired with a DW SS EPI sequence called $e p 2 d \_d l f f p 2 \_t r i g g$, using 2D-PACE and the following parameter settings TR varıed from 3,267 to $4,209 \mathrm{~ms}$ (depending on the respiratory cycle of each subject), $\mathrm{TE}=94 \mathrm{~ms}$, eighteen $10 \mathrm{~mm}$

thick axial slices were acquired with an interslice gap of $25 \mathrm{~mm}, \mathrm{NEX}=1, \mathrm{FOV}$ $=380 / 400 \mathrm{~mm}($ women $/ \mathrm{men})$, phase $\mathrm{FOV}=75 \%$, matrix size $=144 \times 192$, 1 PAT GRAPPA reduction factor $=2$, and the imaging time required for the $13 b$-values and 18 slices was $124 \mathrm{~s}$ (note that GRAPPA is Siemens acronym for parallel 
Imaging performed in $\mathrm{k}$-space) The total ımage acquisition tıme was between 3 and 4 minutes depending on each volunteer's respiratory cycle With this protocol the voxel volume was $2 \times 2 \times 10 \mathrm{~mm}^{3}=40 \mathrm{~mm}^{3}$ and $21 \times 21 \times 10 \mathrm{~mm}^{3}=44 \mathrm{~mm}^{3}$ for women and men, respectively, and the number of slices that could be acquired was not limited by the subject's ability to hold his or her breath which allowed for complete liver coverage For each slice, $13 \mathrm{DW}$ images were obtained with $b$ values of $0,50,100,150,200,250,300,350,400,450,500,650$ and $800 \mathrm{~s} / \mathrm{mm}^{2}$ The diffusion gradients were applied in the slice selection direction (head-to-foot) in all cases Spectral Adiabatic Inversion Recovery (SPAIR) fat supression was used to avold chemical shift artifacts

\subsubsection{Image Analysis}

Data analysis was based on the IVIM model [76], which considers water diffusion and blood microcirculation to be separate processes with slow exchange between these two compartments (see also Section 23 3) The signal decay for this model is biexponential

$$
S / S_{0}=(1-f) \exp (-b D)+f \exp \left(-b D^{*}\right)
$$

where $S$ is the measured DW signal, $S_{0}$ is the $b=0$ signal, $D$ and $D^{*}$ are the diffusion and pseudo-diffusion coefficients, respectively, and $f$ is the perfusion fraction, which represents the fractional volume occupıed in the voxel by MR visible spins moving in the capillaries of the tissue $D^{*}$, which is sometimes also called the perfusion coefficient, is related to the displacement of water molecules In the microvasculature of the tissue during the measurement 


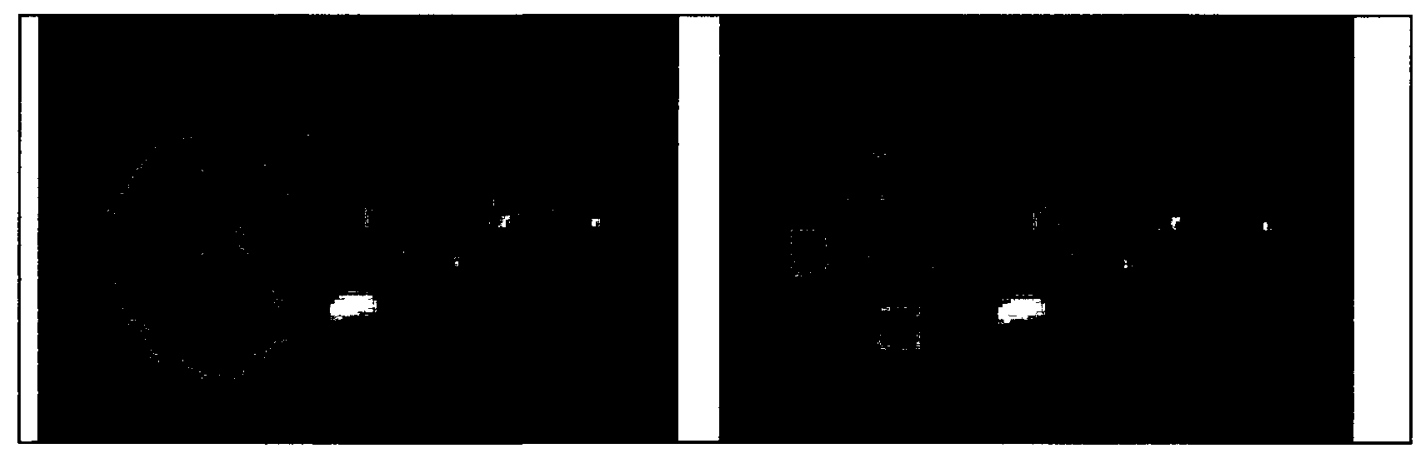

Fig. 3.2. The whole right side of the liver (WRL) ROI is shown in red and the periphery of the night side of the liver (PRL) ROIs are shown in blue All ROIs have been drawn on anatomical images for a healthy liver (volunteer 16)

Analysis was performed in the same manner for both the $\mathrm{BH}$ and $\mathrm{FB}$ protocols Three square 100-pixel ROIs were investigated for each slice of the liver (see Fig 32 ) The mean and standard deviation (SD) of the pixel intensities was computed for each ROI and the mean values were used to generate the diffusion decays The ROIs were drawn peripherally in anterior, mediolateral and posterior regions of the right lobe of the liver, with larger blood vessels being avoided as much as possible The decay was biexponential for all ROIs investigated for both protocols (see, for example, Fig 3 3) The LevenbergMarquardt nonlınear least-squares method was used to fit the decays to Eq 31 to obtain values for the IVIM parameters, $D, f$ and $D^{*}$ The values plotted in the graphs represent the parameter values obtained for each ROI averaged over all slices for each volunteer

Another ROI, which we call the penphery of the nght lobe (PRL) ROI is generated from the union of these three square 100-p1xel ROIs The inclusion of this ROI is justified and discussed further below 


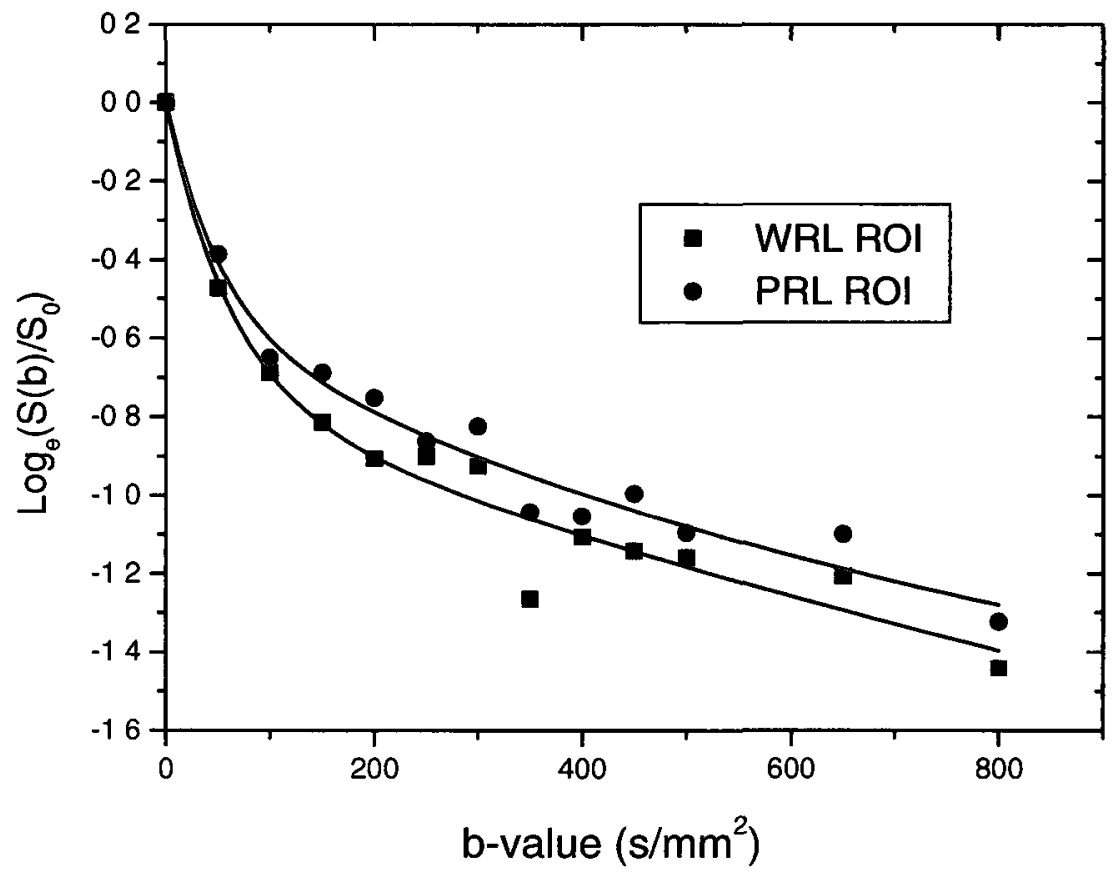

Fig. 3.3. Typical diffusion decays for the WRL-ROI (black) and the PRL-ROI (red) (volunteer 16)

Separately, for each slice, a large ROI was drawn to encompass as much of the night lobe of the liver as possible (see Fig 32) The mean and SD of the pixel intensities were computed for this whole nght liver (WRL) ROI and the mean values were used to generate diffusion decays which were again b1exponential The Levenberg-Marquardt nonlınear least-squares method was used to fit the decays to Eq 31 to obtain values for $D, f$ and $D^{*}$ The graphs in Figs 35 to 37 show the IVIM parameter values obtained for each WRL-ROI averaged over all slices for each subject $D, D^{*}$ and $f$ maps were also calculated on a pixelby-pixel basis for thıs WRL-ROI using a Levenberg-Marquardt fit to Eq 31 
These maps were overlard in colour onto the corresponding $b=0 \mathrm{~s} / \mathrm{mm}^{2}$ anatomical 1mages (see Fig 34 )

All image analysis was performed using software written in the MRI lab at the Ottawa Hospital using IDL (ITT Visual Information Solutions, Boulder, CO)
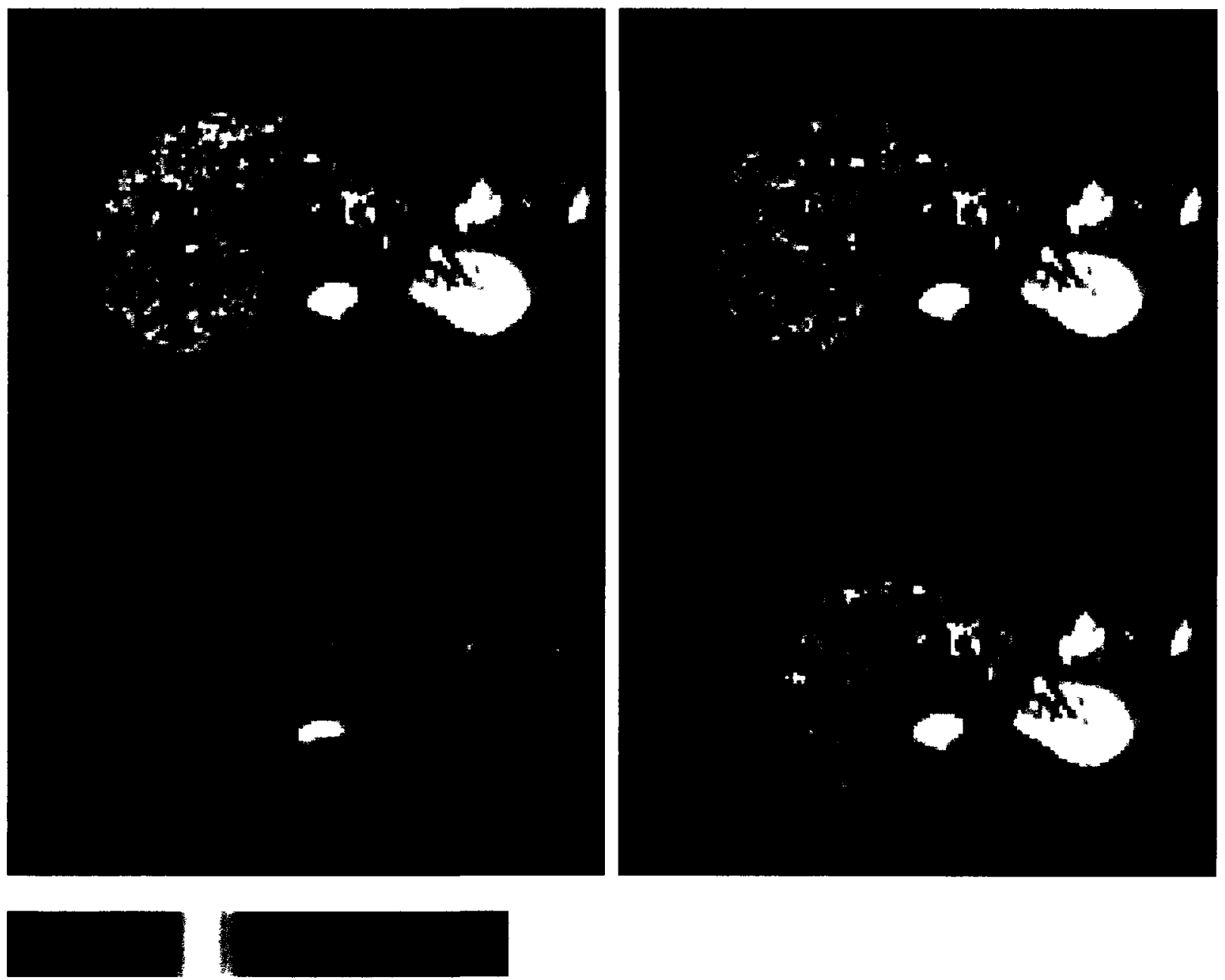

Fig. 3.4. An anatomical image for a normal liver (shice 19, volunteer 16) 1s shown on the lower left and $f$ (upper left), $D$ (upper right) and $D^{*}$ (lower right)-maps color overlaid onto the anatomical image, are shown as indicated The color table ranges for the maps are 1) $0-100 \%$ for the $f$-map, 2) $(0-2) \mu \mathrm{m}^{2} / \mathrm{ms}$ for the $D$ map and 3) $(0-200) \mu \mathrm{m}^{2} / \mathrm{ms}$ for the $D^{*}$-map 


\subsection{Results}

DW images were obtained for 11 volunteers 3 with the $\mathrm{BH}$-protocol and 8 with the FB-protocol using a NRT-DW sequence Only the right hepatic lobe was evaluated It was not possible to perform reliable analyses of the other lobes due to motion artifacts caused by cardiac motion

The presence in the images of chemical shift artifacts from fat in the abdominal wall was a significant problem for the BH-protocol, even though spectral fat saturation was used to suppress the fat signal These artifacts were observed in all BH-subjects and this precluded quantitative investigation for most of the slices In total 10 subjects were scanned with this protocol but only 3 data sets could be used Furthermore, the analysis had to be limited to 4 slices for each of these 3 subjects due to the presence of artifacts in the outer slices

With the NRT-DW sequence, fat signal suppression was done using the SPAIR technique with far better results compared to the fat saturation technique used with the BH-protocol Chemical shift artifacts and/or motion artifacts were observed in just a few of the subjects investigated, however, these artıfacts, if they were seen at all, were not problematic since they was present only on the first slices of the acquisition - slices that did not contain any of the liver

Diffusion decays were generated using the mean values calculated over the corresponding ROI for each $b$-value In all cases the diffusion decay was clearly bi-exponential The decays did not need rectified noise bias correction since the signal-to-noise ratio (SNR) for the highest $b$-values (1 e lowest signal) was always greater than 31 for the images obtained with the BH-protocol and 
greater than 71 for the FB-protocol For the $b=0$ images the SNR was typically about 91 or higher for the BH-protocol and 241 for the FB-protocol The decays were fit to Eq 31 as described above for both breathing protocols and the values of $D, f$, and $D^{*}$ were obtained for 3 square 100-pixel ROIs located in anterior, mediolateral and posterior positions of the liver's right lobe and for the WRLROI

All statıstical analyses were done using ROIs SPSS software was used for statistical computations All ROI distributions were assumed to be normal for these initial analyses and therefore the means and standard deviations of the data were reported A $t$-test was used to compare the means between WRL-ROI and PRL-ROI as well as with values reported in the literature Not having the literature data in our possession, we could not be sure of the form of the distributions but we made the assumption that their data are also normally distributed Mean comparison tests were performed and a two sided asymptotically significant $p$-value was reported in all cases Statıstical sıgnificance was defined as $p<005$ However the assumption of normality was re-examined in a secondary analysis by calculating the skewness and kurtosis for the data sets and by performing a Shapiro-Wilk test of normality With this secondary analysis, it was found that the $D^{*}$ parameter distributions did not obey the condition of normality and a Mann-Whitney non-parametric test was therefore used in these cases These results are found in Appendix A

No consistent systematic changes of the IVIM parameters were observed with slice position for any of the subjects or ROIs considered For some of the 
subjects there was an indication of an interslice variation for some of the parameters but this trend could not be discerned beyond experimental error Therefore, the values in all of the tables and figures of this chapter are averaged over all slices

The IVIM parameter values for the three 100-pixel ROIs showed excellent intra-subject agreement, both within the same slice and across slices, for each subject and for both breathing protocols (see Figs 35 to 37 and Tables 31 to 33 ) The scatter for $D^{*}$ was a little bit larger but no systematic changes were observed This good agreement suggests that the IVIM parameters are the same for the regions of the right lobe investigated with the square 100-pixel ROIs and, by extension, to all peripheral areas of the right lobe With this result as justification, the IVIM parameters were also computed as the mean over the 3 intra-slice 100-p1xel ROIs and over all slices These mean values are labeled as the PRL-ROI values in the figures and tables of this section where PRL stands for Periphery of the Right Lobe

The results obtained with the WRL-ROI also showed excellent intrasubject agreement for each slice and for both breathing protocols Thus, the values reported here are again averaged over all slices The results for the WRL-ROI also agree very well with the results from the 100-pixel ROIs and the PRL-ROI 
Table 3.1. Mean $D, D^{*}$, and $f$ values for the right side of the liver for the FB-protocol

\begin{tabular}{ccccc}
\hline IVIM Parameter & WRL-ROI & CV & PRL-ROI & CV \\
\hline$D\left(\mu \mathrm{m}^{2} / \mathrm{ms}\right)$ & $110 \pm 016$ & 015 & $106 \pm 022$ & 021 \\
$D^{*}\left(\mu \mathrm{m}^{2} / \mathrm{ms}\right)$ & $46 \pm 17$ & 037 & $45 \pm 19$ & 042 \\
$f(\%)$ & $440 \pm 69$ & 016 & $423 \pm 88$ & 021 \\
\hline
\end{tabular}

Note Data are reported as mean \pm SD and CV, the coefficient of varıation, is defined as SD/mean

Table 3.2. Mean $D, D^{*}$, and $f$ values for the right side of the liver for the BH-protocol

\begin{tabular}{ccccc}
\hline IVIM Parameter & WRL-ROI & CV & PRL-ROI & CV \\
\hline$D\left(\mu \mathrm{m}^{2} / \mathrm{ms}\right)$ & $113 \pm 022$ & 019 & $104 \pm 028$ & 027 \\
$D^{*}\left(\mu \mathrm{m}^{2} / \mathrm{ms}\right)$ & $70 \pm 19$ & 027 & $67 \pm 50$ & 075 \\
$f(\%)$ & $34 \pm 11$ & 032 & $29 \pm 12$ & 041 \\
\hline
\end{tabular}

Note Data are reported as mean \pm SD and CV, the coefficient of variation, is defined as SD/mean

Table 33 Mean $D, D^{*}$, and $f$ values for the right side of the liver for the FB-protocol when a $t$-test was performed, and a two tailed $p$-value is reported

\begin{tabular}{cccc}
\hline IVIM Parameter & $\begin{array}{c}\text { WP-ROI } \\
(\mathrm{N}=8)\end{array}$ & $\begin{array}{c}\text { PRL-ROI } \\
(\mathrm{N}=8)\end{array}$ & $p$-value \\
\hline$D\left(\mu \mathrm{m}^{2} / \mathrm{ms}\right)$ & $110 \pm 016$ & $106 \pm 022$ & 0238 \\
$D^{*}\left(\mu \mathrm{m}^{2} / \mathrm{ms}\right)$ & $46 \pm 17$ & $45 \pm 19$ & 0937 \\
$f(\%)$ & $440 \pm 69$ & $423 \pm 88$ & 0437 \\
\hline
\end{tabular}

Note Data are reported as mean \pm SD (if $p$-value $<005$, then results are statistically different)

\subsubsection{Perfusion Fraction}

The variation of $f$ for the 100-pixel ROIs, the PRL-ROI and the WRL-ROI, averaged over all slices in each case, is shown in Fig 35 For both breathing protocols there is very good intra-subject agreement For the FB-protocol there is also very good inter-subject agreement for the $f$ values for all ROI types The mean $f$ value, averaged over all FB subjects, for the WRL-ROI, $f=(440 \pm 69) \%$, is in very good agreement with the mean value calculated for the PRL-ROI, $f=$ $(423 \pm 88) \%$ For the FB-protocol, all but one value fell within one SD of the 
mean calculated for this group of subjects, the exception being the WRL-ROI value for volunteer 7 Since all subjects were apparently healthy, it is not clear why this one value was lower than the rest Results in the literature have shown that exercise and/or fastıng changes the blood volume in liver, exercise actually decreasing it [96] We did not record if the subjects were fasting or have exercised before the scan, so we cannot make any assumption on this matter

Although there is very good inter-subject agreement for the FB-protocol, this is not the case for the BH-protocol where the $f$ value for volunteer 2 is considerably higher than it is for the other two, especially for the WRL-ROI However, the value obtained for volunteer 2 appears to be in better agreement with the FB-protocol results than the other two, suggesting that this may be the more reliable value The mean $f$-value calculated for the PRL-ROI $(29 \pm 12) \%$ is in good agreement with the value calculated for the WRL-ROI $(34 \pm 11) \%$ with the BH-protocol The observed variation is within the reported measurement error although, since only 3 subjects were scanned for this data set, the standard deviation calculated over the group of 3 subjects may not be a good representation of the true experimental error

There is an appreciable difference between the $f$ values obtained with the two breathıng techniques under investigation (see Tables 31 and 32) The $f$ values obtained with the FB-protocol are much larger than the $f$ values obtained for volunteers 1 and 3 with the BH-protocol, however, the value obtanned for volunteer 2 is in good agreement with the other two subjects The variance is also much lower for the FB-protocol For the WRL-ROI, the coefficient of variation 
(CV) is 032 for the BH-protocol whereas it is only 016 for the FB-protocol showing more precise results The situation for the PRL-ROI is similar, with CV values of 041 and 021 for the $\mathrm{BH}$ and FB protocols, respectively

It should also be noted that the inclusion of smaller $b$-values for the BHprotocol does not appear to have improved the precision of the $f$ values obtained with this technique This is not surprising since $f$ is determined primarily by the large $b$-value part of the decay 

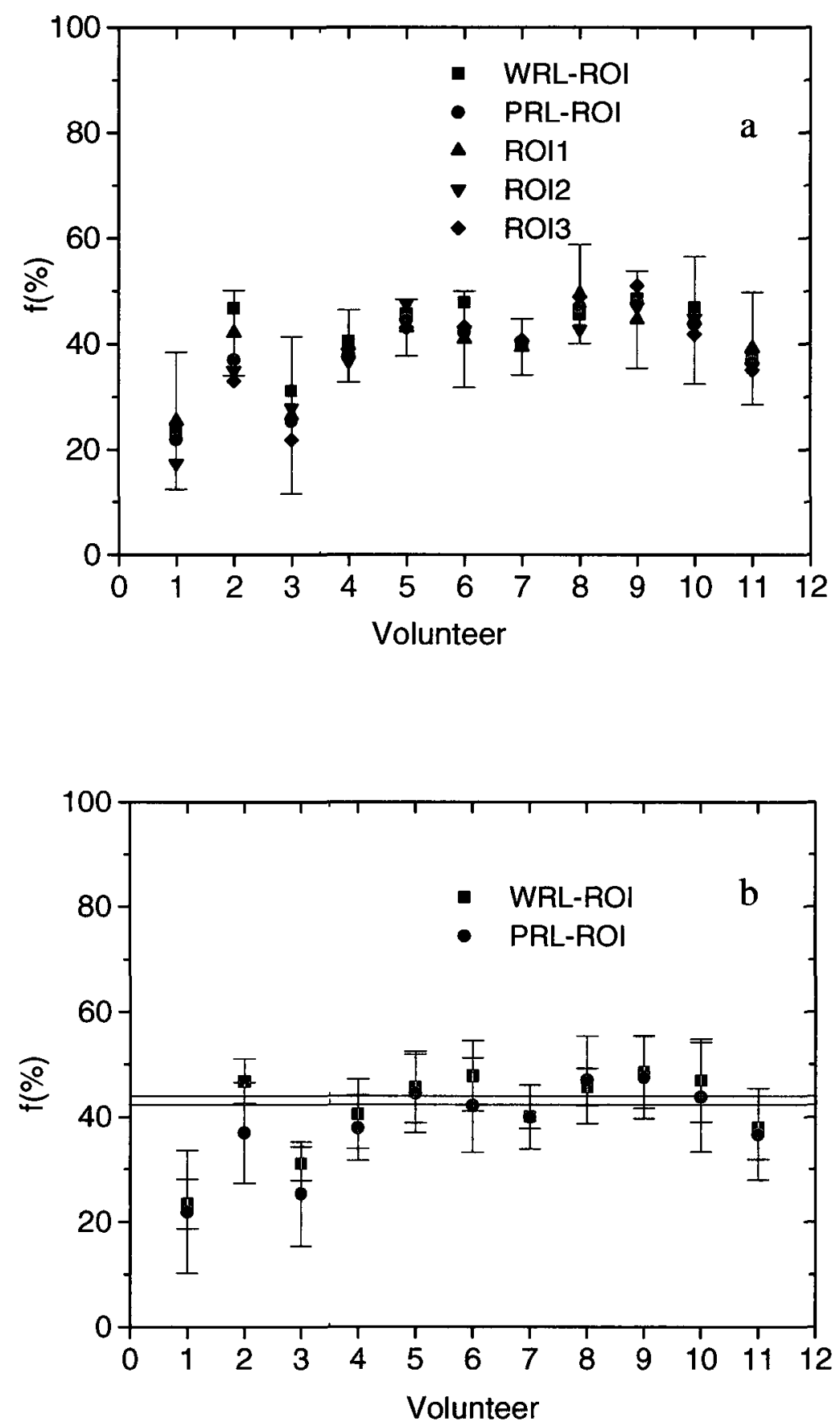

Fig. 3.5. $f$ values (mean $\pm \mathrm{SD}$ ) are shown for the WRL-ROI, the three 100-pixel ROIs and the PRL-ROI, averaged over all slices in each case The BH-protocol was used for volunteers 1 to 3 and the FB-protocol was used for volunteers 4 to 11 The horizontal lines show the mean over all volunteers scanned with the FBprotocol for the PRL-ROI (red) and the WRL-ROI (black) For clanty, error bars are shown in a) for ROI1 only 


\subsubsection{Diffusion Coefficient}

The variation of $D$ for the 100-pixel ROIs, the PRL-ROI and the WRL-ROI, averaged over all shces in each case, is shown in Fig 36 For both breathing protocols there is very good intra-subject agreement The average over all subjects for the BH-protocol gave a mean diffusion coefficient of $D=\left(\begin{array}{lll}1 & 13 \pm\end{array}\right.$ $022) \mu \mathrm{m}^{2} / \mathrm{ms}$ for the WRL-ROI and $D=(104 \pm 028) \mu \mathrm{m}^{2} / \mathrm{ms}$ for the PRL-ROI For the FB-protocol we obtained $D=(110 \pm 016) \mu \mathrm{m}^{2} / \mathrm{ms}$ for the WRL-ROI and $D=(106 \pm 022) \mu \mathrm{m}^{2} / \mathrm{ms}$ for the PRL-ROI The data also showed good intersubject agreement for both breathing protocols The values for all but one subject fell within one SD of the mean for the protocol, the exception being the WRLROI value for volunteer 5 Since all subjects were apparently healthy, it is not clear why this one value was higher than the rest

No significant difference was observed between the $D$ values for the WRL-ROI and the PRL-ROI with the FB protocol and a similar behaviour was observed for BH breathing protocol (see $p$-values in Table 3 3) The WRL value was higher than the PRL value for all subjects, however, the $D$ values for these two ROIs were all within one SD of each other (Fig $36 \mathrm{~b}$ ) While the $D$ values obtained with the two breathing protocols are in very good agreement, it should be noted that there is a $25-30 \%$ improvement in the precision when the FBprotocol is used (see Tables 31 and 3 2) For the WRL-ROI, CV equals 019 for the BH-protocol whereas it is only 015 for the FB-protocol The situation for the PRL-ROI is very similar with CV values of 027 and 021 for the BH and FB protocols, respectively 
The 100-pixel ROI analysis showed that $D$ does not vary significantly within a slice as long as the ROI positioning is carefully done so as to avord large blood vessels This conclusion is supported, for both breathing protocols, by the $D$-maps, an example of which is shown in Fig 34 (upper right) This is the expected behaviour since $D$ is not related to the microcirculation in the tissue, $D$ is governed by tissue structure Diffusion dominates the higher range of $b$-values on the decay, where capillary blood flow effects do not contribute

The inclusion of smaller $b$-values for the BH-protocol does not appear to have improved the precision of the $D$ values obtained with this technique This is not surprising since $D$ is determined primarily by the large $b$-value part of the decay 

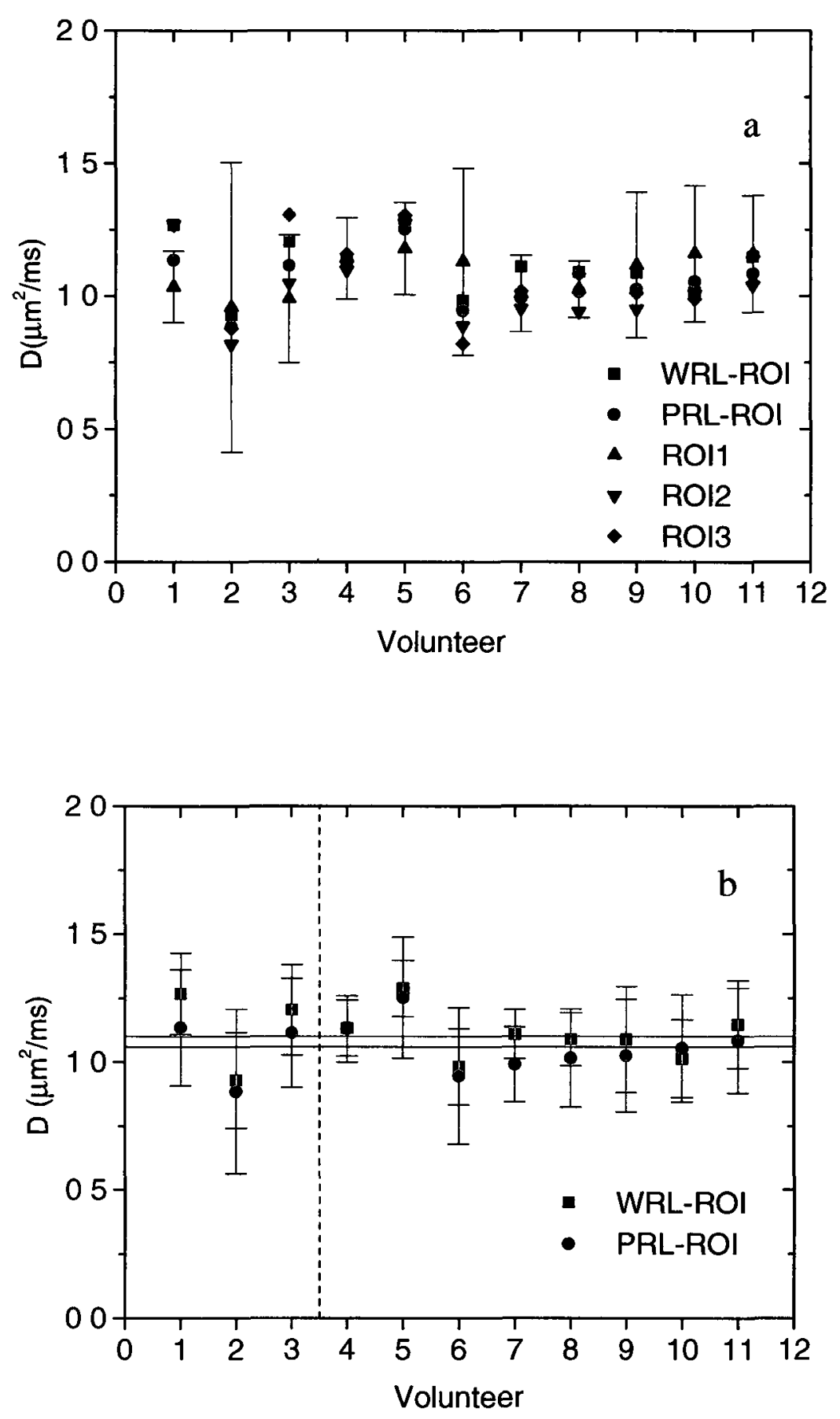

Fig. 3.6. $D$ values (mean \pm SD) are shown for the WRL-ROI, the three 100-pixel ROIs and the PRL-ROI, averaged over all slices in each case The BH-protocol was used for volunteers 1 to 3 and the FB-protocol was used for volunteers 4 to 11 The horizontal lines show the mean over all volunteers scanned with the FBprotocol for the PRL-ROI (red) and the WRL-ROI (black) For clanty, error bars are shown in a) for ROI1 only 


\subsubsection{Pseudo-Diffusion Coefficient}

The variation of $D^{*}$ for the 100-pixel ROIs, the PRL-ROI and the WRL-ROI, averaged over all slices in each case, is shown in Fig 37 For both breathing protocols there is good intra-subject agreement For the FB-protocol, the values of $D^{*}$, averaged over all subjects, were $D^{*}=(46 \pm 17) \mu \mathrm{m}^{2} / \mathrm{ms}$ and $D^{*}=(45 \pm$ 19) $\mu \mathrm{m}^{2} / \mathrm{ms}$ for the WRL-ROI and the PRL-ROI, respectively The corresponding values for the BH-protocol were $D^{*}=(70 \pm 19) \mu \mathrm{m}^{2} / \mathrm{ms}$ and $D^{*}=$ $(67 \pm 50) \mu \mathrm{m}^{2} / \mathrm{ms}$, respectively The values obtained with these two ROIs are in very good agreement for the same breathing protocol

The data showed good inter-subject agreement for both breathing protocols, particularly for the FB-protocol (see $p$-values in Table 3 3) All but one value fell within one SD of the group mean, the exception being volunteer 1 for the BH-protocol when the PRL-ROI was considered Since all volunteers were apparently healthy, it is not clear why this one value was higher than the rest

There is a notable difference between the $D^{*}$ values obtained with the two breathing techniques (see Tables 31 and 32 ) The three $D^{*}$ values for the $\mathrm{BH}$ protocol are all higher than the $D^{*}$ values for the FB-protocol The variance is also much higher for the BH-protocol For the PRL-ROI, the coefficient of variation (CV) is 075 for the $\mathrm{BH}$-protocol whereas it is only 042 for the FBprotocol - a very significant improvement The situation for the WRL-ROI, however, is reversed with $\mathrm{CV}$ values of 027 and 037 for the $\mathrm{BH}$ and FB protocols, respectively The elevated CV value for the FB-protocol, in this case, 
may reflect the expected variation in the microvascular blood flow pattern across the liver rather than increased experimental error

The inclusion of smaller $b$-values for the BH-protocol does not appear to have improved the precision of the $D^{*}$ values obtained with this technique This 1s a bit surprising since $D^{*}$ is determined primarily from the low $b$-value part of the decay Presumably, errors due to other problems with the BH-protocol cancel out any gain in precision from these additional low $b$-value points

The variation of $D^{*}$ across the liver on the color parametric maps (Fig $34 \mathrm{~d}$ ) is consistent with the anatomy of the liver Areas with higher $D^{*}$ values can be seen around larger blood vessels $\sim$ brighter areas on Fig 34 (lower left), where, since they are collecting or spreading blood, a higher blood flow is expected (see Section 26 for a review of the anatomy of the liver and its hemodynamic hierarchy) and a constant behaviour is observed for the rest of the liver 

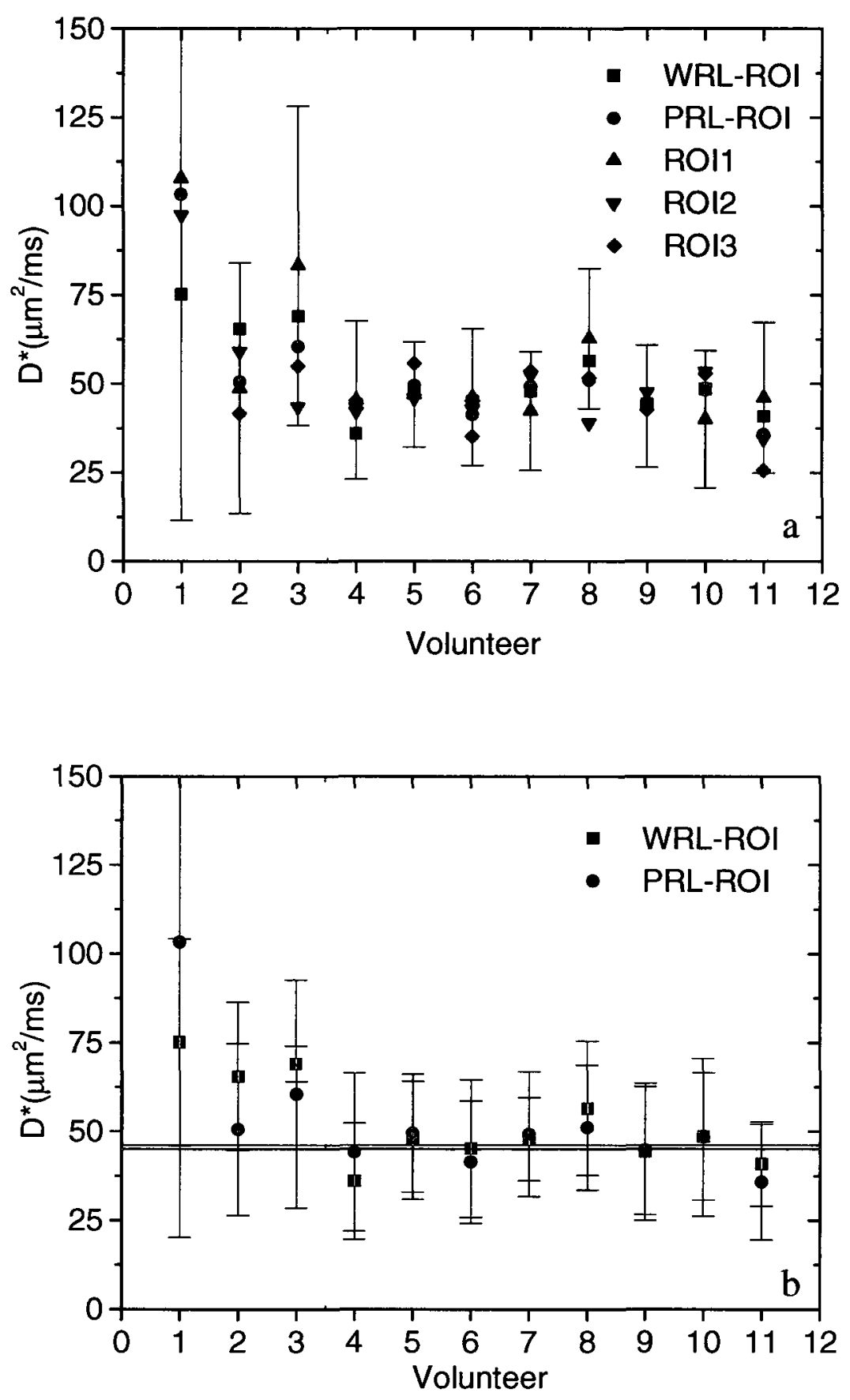

Fig. 3.7. $D^{*}$ values (mean $\pm \mathrm{SD}$ ) for the WRL-ROI, the three 100-pixel ROIs and the PRL-ROI, averaged over all slices in each case The BH-protocol was used for volunteers 1 to 3 and the FB-protocol was used for volunteers 4 to 11 The horizontal lines show the mean over all volunteers scanned with the FB-protocol for the PRL-ROI (red) and the WRL-ROI (black) For clanty, error bars are shown in a) for ROI1 only 


\subsection{Discussion}

In this chapter the movement of blood in the microvasculature of the liver, ıncluding arterioles, capillaries and venules, was investigated using the IVIM model [5] and an assessment of the values of the fit parameters $f, D$ and $D^{*}$ was made The active microvascular fractional volume, $f$, also known as the perfusion fraction, represents the ratio of the volume of MR visible water moving in the microvascular compartment (e g water in blood cells, plasma, etc) to the total volume of MR visible water present in the voxel The pseudo-diffusion coefficient, $D^{*}$, is the exponential decay constant for the MR signal attenuation induced by the displacement of water in the microvasculature The capillaries in the liver are assumed to follow a pseudo-random course such that motion of the microvascular water can be described in terms of a random walk model Macroscopically ( 1 e on the scale of a typical voxel), this motion appears very much like a diffusion process Thus, $f$ is a measure of how much water is present in the microvasculature of the tissue and $D^{*}$ is related to the speed of the circulating blood and the length of the randomly onented capillary segments [76]) For the size of the voxel investigated in our studies $\left(90 \mathrm{~mm}^{3}\right.$ with the BHprotocol and $40 \mathrm{~mm}^{3}$ and $44 \mathrm{~mm}^{3}$ for women and men, respectively, with the FBprotocol) a very large number of randomly oriented arterioles, venules and capillary segments are to be expected to exist in a single voxel (see Section 26 for a review of liver anatomy and physiology)

Many hepatic pathologies cause changes to the microvasculature of the liver With fibrotic disease the vascularity of the tissue decreases whereas with 
cancer, angiogenesis causes increased vascularity at the site of the lesion The values of $f$ and $D^{*}$ can be expected to change accordingly and should be sensitive indicators of these forms of hepatic pathology Moreover, accurate values of these IVIM parameters should indicate the degree to which the microvasculature has been affected by the pathology and could be specific for certain hepatic pathologies [95] In this thesis we present values of these parameters for healthy liver tissue Deviations from these values in patients can be expected to be an indication of the presence of disease Further study is required to determine the sensitivity and specificity of the IVIM parameters but the work presented here is an important first step

The diffusion coefficient, $D$, is not related to microcirculation in the tissue It is related to the ability of the extravascular water (1 e intracellular and interstitial water) to diffuse [66] This, in turn, is determined primarily by the cellularity of the tissue The diffusion coefficient can be expected to decrease, relatıve to normal liver, in fibrotıc tıssue, where the fibres presumably inhibit diffusion, and in cancerous lesions where cell crowding restricts diffusion Reliable quantitatıve values for $D$ should be sensitive to the presence of pathology in hepatic tissue but it is unclear if it will be a useful parameter for discrimınatıng between different pathologies Further study is required to answer this question

In the work presented here, two different breathing protocols (breath hold and free breathing) were used and the obtained IVIM parameter values were compared For each of these methods, ROIs were chosen in three distinct ways 1) three 100-pixel square ROIs were placed at locations around the periphery of 
the right hepatıc lobe (anteriorly, mediolaterally and posteriorly) to sample different locations withın the liver, 2) a ROI which we call the periphery of the right lobe (PRL) ROI was formed from the union of the 3 100-pixel ROIs (see Section 33 for justification) and 3) a large ROI was selected to cover as much of the right hepatic lobe as possible The diffusion decays were generated from the means of these ROIs for each $b$-value Components of the signal decay corresponding to water that is diffusing only and water that is both circulating and diffusing in the microvasculature were identified for all ROI choices and both breathing protocols These DWI measurements provide noninvasive quantification of water diffusion and blood circulation in the microvascular network of liver tissue $i n$ vivo

Parametric maps were also generated for the WRL-ROI for each of the IVIM parameters The distribution of the parameter values across these maps was, in general, rather unıform, however, there was a recognizable pattern with both $f$ and $D^{*}$ which is consistent with the composition of the liver tissue The $f$ maps (see Fig 34 upper left) show a clear gradıent from smaller values near larger blood vessels (which can be seen as brighter areas in Fig 34 lower left), to larger values in peripheral regions where capillary density is higher Major blood vessels are consistently depicted in the $f$-maps as either a small blue region or a blue ring around a white spot The $D^{*}$-maps (see Fig 34 lower right) showed elevated values at locations in and around larger blood vessels where blood flow 1s expected to be higher The $D$-maps (see Fig 34 upper right) showed a small variation of values across the liver but this variation does not appear to be 
correlated with liver anatomy or physiology It is more likely simply a reflection of the range of $D$ values that can be expected for such a measurement

These parametric maps show great promise as visual tools for the radiologist but they do not lend themselves to easy quantitative analysis so they will not be considered further in this chapter Instead we decided to concentrate our efforts on region of interest analyses

One potential deficiency of the study is that the diffusion sensitivity gradients were only applied in the inferior-superior direction It would have been nice to measure the diffusion in other directions as well However this would have extended the exam time considerably and, given the composition of the liver, a directional dependence of the IVIM parameters was not anticipated

\subsubsection{Comparison of the IVIM Parameters for Different ROIs}

The results obtained with the WRL-ROI for different slices were in good agreement, both within the same slice and across slices (1 e low intra-subject variation), for both breathing protocols Thus, the values reported here are the average over all slices This behavior is also apparent in the IVIM parametric maps, as noted above The intra-subject agreement for each subject with the three 100-pixel ROIs was also good, for both breathing protocols and all three IVIM parameters This suggests that the IVIM parameters are the same for the peripheral regions of the right lobe of the liver investigated and, by extension, to all perıpheral areas of the nght lobe This observation validates our use of the PRL-ROI 
To within the experimental error of the measurements presented here, the same IVIM parameter values are obtaned for the PRL-ROI and the WRL-ROI, even though the positioning of the three 100 -pixel ROIs was done very carefully to avold large vessels while no such precaution was taken for the ROI drawn to encompass the whole right lobe Presumably the explanation for this is that in the larger WRL-ROI there is a relatively small proportion of the total number of voxels which contain large vessels, with the consequence that these vessels have a relatively small effect on the diffusion decay for this ROI These vessels would likely have an important effect on smaller ROIs (e g 100 pixels), where fewer pixels are involved in the computation of the mean, but not on ROIs as large as the WRL-ROI

It should be noted that the reported range of WRL-ROI IVIM values, partıcularly $f$ and $D^{*}$, is determıned partly by experimental error and partly by changes in the composition of the liver tissue at different locations within the ROI The observed smaller SD found for all parameters with the WRL-ROI suggests that experimental error is the dominant contribution in each case since the number of pixels in the WRL-ROI from which the mean and SD were calculated is much larger than 300 pixels and the larger tissue variation across the WRL-ROI relative to the PRL-ROI would cause SD to increase, not decrease For these reasons and the fact that, based on the measurements reported here, the IVIM parameters are the same (to withın experimental error) for all parts of the right lobe of the liver, the WRL-ROI appears to be a better, more robust choice (See Table 3 3) 


\subsubsection{Breath Hold Protocol versus Free Breathing Protocol}

The agreement between the results obtained with the two breathing protocols was acceptable in that the agreement was within experimental error However, the BH-protocol and/or data set had numerous deficiencies These include 1) strong chemical shift and motion artifacts on many of the source images, 2) a small sample size of only 3 subjects, 3) uncertainty regarding voxel position from one measurement to the next, 4) large variances for both individual experıments and across the group of subjects, 5) the number of slices acquired and the number of $b$-values used were both limited and signal averaging could not be employed since the image acquisition time was restricted to a single breath hold and 6) the scanner hardware used for the experıments was not state-of-the-art

The images acquired with the FB-protocol, which were obtained after the scanner was upgraded to state-of-the-art equipment, were far superior to those acquired using the BH-protocol, all of which were obtained prior to the upgrade Chemical shift and motion artifacts were not apparent on the images acquired on the upgraded scanner It was also possible to use NRT sequences after the upgrade to give increased confidence that the voxels were at the same location for each measurement This option was not avallable on the older system

The intra-subject variance of the data was larger for the BH-protocol than it was for the FB-protocol mainly because the hardware used for the $\mathrm{BH}$ experıments was not as good and NRT sequences were not used The upgraded scanner allowed us to use the SPAIR fat suppression technique which provides far 
better fat suppression This removed the chemical shift and motion artifact problem We also obtained better SNR due to superior rf coll technology Reasons for the small number of subjects for the BH-protocol include 1) this protocol had to be stopped after the MR scanner was upgraded and 2) the results for 7 of the 10 subjects scanned with this protocol had to be rejected due to strong chemical shift and motion artifacts in the images It is gratifying that the results of these two protocols are in agreement to within experimental error but the FBprotocol is clearly far superior

One of the disadvantages of performing the imaging during a breath hold 1s that it restricts the duration of the scan to about $30 \mathrm{~s}$ or less This limits the number of slices that can be acquired and/or the number of averages and $b$-values that can be used Furthermore, for the BH-protocol, even though the subject is holdıng his or her breath, this does not guarantee that the abdomınal organs will remain stationary during a scan, particularly towards the end of the scan when it becomes more difficult for the subject to maintain the breath hold Inaccurate reproducibility of the breath hold for successive scans may also lead to changes in the location of the abdominal organs from one measurement to the next These issues are considerably reduced (if not completely eliminated) when a navigator echo is used to accurately track the motion of the diaphragm while the patient breathes normally and comfortably

The BH-protocol used for the work reported in this chapter did, however, have one potential advantage over the FB-protocol since it was possible to use smaller $b$-values with the BH-protocol The DWI product sequence provided by 
Siemens allows an increment in the $b$-values of $50 \mathrm{~s} / \mathrm{mm}^{2}$ Prior to the upgrade we were able to modify this increment to $10 \mathrm{~s} / \mathrm{mm}^{2}$, under a research agreement with Siemens This allowed the use of $b$-values between 0 and $100 \mathrm{~s} / \mathrm{mm}^{2}$ that were multiples of $10 \mathrm{~s} / \mathrm{mm}^{2}$ instead of $50 \mathrm{~s} / \mathrm{mm}^{2}$ However, following the upgrade, a new research agreement with Siemens was not in place in time to allow the incorporation of this sequence modification into the FB-protocol used here Such a change to the protocol should have little or no effect on the values of $D$ and $f$ obtained since these parameters are determined primarily by the behaviour of the large $b$-value part of the decay ( $1 \mathrm{e}$ they are related to the slope and y-1ntercept, respectively, of the long component when plotted on a sem1logarithmic plot) However, inclusion of smaller $b$-values into the protocol could improve the precision, of the $D^{*}$ values obtained since this is the part of the decay that is most affected by the microcirculation

The intra and inter-subject variability for all IVIM parameters, including $D^{*}$, is higher for the BH-protocol suggesting that the deficiencies of the protocol and the hardware used for these measurements outweigh the benefit of having small $b$-values Another factor that contributed to the reduced intra-subject variance for the FB-protocol was the fact that, since it was possible to acquire more slices with this protocol, there were more slices avallable to include in the computation of the mean and SD With the BH-protocol it was only possible to get 4 slices whereas with the FB-protocol the number of slices ranged from 6 to 10 , depending on the size of the subject's liver It seems reasonable that the 
addition of more $b$-values between 0 and $100 \mathrm{~s} / \mathrm{mm}^{2}$ would improve the FBprotocol but the results presented here do not support or refute this assumption

The reduced number of slices for the BH-protocol also means that it was not possible to cover the whole right lobe of the liver Thus, for the BH-protocol, the average over all slices gives, in principle, values that reflect the average for the shices measured and not necessarily the right lobe as a whole Although variation of the IVIM parameters from shice to slice was found to be relatively small for healthy liver, this may not be the case with diseased tissue In this case, coverage of the whole liver may be a vital requirement for the protocol

Overall, the FB-protocol appears to be far superior to the BH-protocol However, the mean $D^{*}$ values obtained with the BH-protocol are in better agreement with values reported in the literature This will be discussed in more detall below

For the FB-protocol the results showed good inter-subject agreement for all parameters All but two values fell within one SD of the mean for this group of subjects, the exceptions being the WRL-ROI $D$-value for volunteer 5 and the WRL-ROI $f$-value for volunteer 7 Since all subjects were apparently healthy, it is not clear why these values were higher and lower, respectively, than the rest The inter-subject variability for the FB-protocol was considerably better than for the BH-protocol This can be seen by inspection from the figures in Section 33 and from the CV values given in Tables 31 and 32 It should also be noted that the variation of the SD from subject to subject was much smaller for the FBprotocol than it was for the BH-protocol 
If the inter-subject variability for the BH-protocol is determined using the mean and SD for the group of 3 subjects then all parameters fell within one SD of the mean However, this is probably not a fair test since, with such a small number of subjects, data points that should be outliers will have a strong enough effect on the computed SD that they may not be seen as outlers If the average SD for the individual measurements is considered instead, then it can be seen, by inspection, that all of the PRL-ROI values are approximately within one SD In other words, there is an overlap of the range of values determined by the error bars for the individual experiments (see Figs 35 to 37 )

For the WRL-ROI values the ranges determined by the error bars for $D$ and $D^{*}$ again overlap for all three subjects but for the $f$ values, where the error bars are much smaller, none of the values overlap

These observations suggest that the inter-subject variability for the BHprotocol is acceptable for all parameters (except possibly $f$ for the WRL- ROI) but this has to be tempered by the fact that the experimental errors for the $\mathrm{BH}$ measurements were relatively large for all values (except $f$ for the WRL-ROI) The inter-subject variability for the BH-protocol is reasonable but it is considerably worse than for the FB-protocol

From the accuracy point of view, the perfusion fraction values that we obtained are close to the blood volume in liver which is believed to represent about $30 \%$ of the total hepatic volume The arterial system acts as a buffer in maintaining the intrahepatic pressures, but the blood volume will vary as a 
function of these pressure gradients The values we obtained for $f$ for both breathing protocols are shown in Table 31 and Table $32[96,97]$

\subsubsection{Comparison with IVIM Parameter Values in the Literature}

To our knowledge, there are only two other studies that have investigated the IVIM parameters for the liver These studies both used a SS EPI DW sequence but they used different breathıng protocols Yamada et al [12] used a $\mathrm{BH}$ technique while Lucianı et al [9] used a FB technique with a pneumatic belt for respiratory triggering There have been no reports of IVIM studies of the liver where an NRT sequence was used, and there were no studies that report IVIM parameters on whole right liver During the writing of the thesis one paper [95] was published where an NRT sequence and a similar method were used However the data analysis and the fittıng procedures are different from ours A comparison of the IVIM parameters from these studies with the values reported here is given in Tables 34 and 35 Unfortunately, neither of these reports provided values for all of their experımental parameters, makıng a direct comparison with our work difficult 
Table 3.4. IVIM parameter values for the right side of the liver measured with our FBprotocol compared with those obtained by Lucianı et al [9] using a pneumatic belt RT sequence

\begin{tabular}{ccccccc}
\hline $\begin{array}{c}\text { IVIM } \\
\text { Parameter }\end{array}$ & $\begin{array}{c}\text { WRL-ROI } \\
(\mathrm{N}=8)\end{array}$ & $\mathrm{CV}$ & $\begin{array}{c}\text { PRL-ROI } \\
(\mathrm{N}=8)\end{array}$ & $\mathrm{CV}$ & $\begin{array}{c}\text { Luclanı et al } \\
\sim 13 \text {-pixel ROI } \\
(\mathrm{N}=37)\end{array}$ & $\mathrm{CV}$ \\
\hline$D\left(\mu \mathrm{m}^{2} / \mathrm{ms}\right)$ & $110 \pm 016$ & 015 & $106 \pm 022$ & 021 & $116 \pm 09$ & 078 \\
$D^{*}\left(\mu \mathrm{m}^{2} / \mathrm{ms}\right)$ & $46 \pm 17$ & 037 & $45 \pm 19$ & 042 & $851 \pm 27$ & 032 \\
$f(\%)$ & $440 \pm 69$ & 016 & $423 \pm 88$ & 021 & $26 \pm 7$ & 027 \\
\hline
\end{tabular}

Note Data are mean \pm SD Our mean values were obtained by averaging over all subjects for both the WRL-ROI and the PRL-ROI

Table 3.5 IVIM parameter values for the right side of the liver measured with our BHprotocol compared with those obtained by Yamada et al [12] using their BH-protocol

\begin{tabular}{ccccccc}
$\begin{array}{c}\text { IVIM } \\
\text { Parameter }\end{array}$ & $\begin{array}{c}\text { WRL-ROI } \\
(\mathrm{N}=3)\end{array}$ & CV & $\begin{array}{c}\text { PRL-ROI } \\
(\mathrm{N}=3)\end{array}$ & CV & $\begin{array}{c}\text { Yamada et al } \\
100 \text {-pIxel ROls } \\
(\mathrm{N}=47)\end{array}$ & $\mathrm{CV}$ \\
\hline$D\left(\mu \mathrm{m}^{2} / \mathrm{ms}\right)$ & $113 \pm 022$ & 019 & $104 \pm 028$ & 027 & $076 \pm 027$ & 036 \\
$D^{*}\left(\mu \mathrm{m}^{2} / \mathrm{ms}\right)$ & $70 \pm 19$ & 027 & $67 \pm 50$ & 075 & Not reported & - \\
$f(\%)$ & $34 \pm 11$ & 032 & $29 \pm 12$ & 041 & $29 \pm 14$ & 048 \\
\hline
\end{tabular}

Note Data are mean \pm SD Our mean values were obtained by averaging over all subjects for both the WRL-ROI and the PRL-ROI

Table 3.6 Mean IVIM parameter values averaged over the right side of the liver (Ottawa) and the Luciannı [9] data, when a t-test was performed, and a two talled $p$-value is reported

\begin{tabular}{cccccc}
\hline $\begin{array}{c}\text { IVIM } \\
\text { Parameter }\end{array}$ & $\begin{array}{c}\text { LuciannI } \\
(\mathrm{N}=11)\end{array}$ & $\begin{array}{c}\text { WP-ROI } \\
(\mathrm{N}=8)\end{array}$ & $\begin{array}{c}p \text {-value } \\
\text { (Luclannı vs } \\
\text { WP-ROI }\end{array}$ & $\begin{array}{c}\text { PRL-ROI } \\
(\mathrm{N}=8)\end{array}$ & $\begin{array}{c}p \text {-value } \\
\text { (Luciannı } \\
\text { Vs WP-ROI }\end{array}$ \\
\hline$D\left(\mu \mathrm{m}^{2} / \mathrm{ms}\right)$ & $116 \pm 09$ & $110 \pm 016$ & 08554 & $106 \pm 022$ & 07637 \\
$D^{*}\left(\mu \mathrm{m}^{2} / \mathrm{ms}\right)$ & $851 \pm 27$ & $46 \pm 17$ & 00022 & $45 \pm 19$ & 00023 \\
$f(\%)$ & $26 \pm 7$ & $440 \pm 69$ & 00001 & $423 \pm 88$ & 00003 \\
\hline Note Data are reported as mean $\pm \mathrm{SD}$ (if $p$-value $<005$, then results are statistically different)
\end{tabular}

Table 3.7. IVIM parameter values for the right side of the liver measured with our FBprotocol compared with those obtained by Patel et al [95] using an NRT sequence

\begin{tabular}{ccccccc}
\hline $\begin{array}{c}\text { IVIM } \\
\text { Parameter }\end{array}$ & $\begin{array}{c}\text { WRL-ROI } \\
(\mathrm{N}=8)\end{array}$ & $\mathrm{CV}$ & $\begin{array}{c}\text { PRL-ROI } \\
(\mathrm{N}=8)\end{array}$ & $\mathrm{CV}$ & $\begin{array}{c}\text { Patel et al } \\
(\mathrm{N}=14)\end{array}$ & $\mathrm{CV}$ \\
\hline$D\left(\mu \mathrm{m}^{2} / \mathrm{ms}\right)$ & $110 \pm 016$ & 015 & $106 \pm 022$ & 021 & $117 \pm 021$ & 018 \\
$D^{*}\left(\mu \mathrm{m}^{2} / \mathrm{ms}\right)$ & $46 \pm 17$ & 037 & $45 \pm 19$ & 042 & $3961 \pm 1234$ & 031 \\
$f(\%)$ & $440 \pm 69$ & 016 & $423 \pm 88$ & 021 & $3216 \pm 813$ & 025 \\
\hline
\end{tabular}

Note Data are mean \pm SD Our mean values were obtained by averaging over all subjects for both the WRL-ROI and the PRL-ROI 
It is important to mention that, for both of the breathing techniques employed in our measurements, the precision of the $f$ and $D$ values (based on the $\mathrm{CV}$ values given in Tables 34 and 35) is significantly higher than for the previously reported studies and it is comparable for $D^{*}$ This significant improvement we believe to be due primarily to the superior methodology employed in our study

\subsubsection{The IVIM study of Yamada et al.}

Yamada et al [12] studied the application of the IVIM technique to several abdominal tissues including both normal liver and hepatıc lesions They used a breath hold SS EPI DW technique, with TE $=123 \mathrm{~ms}$, a slice thickness of $8 \mathrm{~mm}$ and a set of only $4 b$-values $\left(0,30,300\right.$ and $\left.900 \mathrm{~s} / \mathrm{mm}^{2}\right)$ The number of Investigated slices was not reported They obtained bi-exponential diffusion decays and used IVIM theory to analyse the results The Levenberg-Marquardt nonlinear least-squares algorthm was used to fit the decay and obtain the IVIM parameters, $f, D$ and $D^{*}$, although no $D^{*}$ values were reported The parameters for normal liver were evaluated as the average over 47 subjects and over 3 or 4 circular ROIs contaınıng at least 100 pixels per subject No information is given about the positioning of the ROIs so it is unclear if they attempted to avoid large vessels or if they restricted their investıgation to a partıcular lobe of the liver

The reported mean \pm SD values for their IVIM parameters for healthy subjects are $D=(076 \pm 027) \mu \mathrm{m}^{2} / \mathrm{ms}$ and $f=(29 \pm 14) \%$ where their reported SD values were calculated over the group of subjects and are considered by them 
to be a measure of the inter-subject variability These values are considerably lower than our values obtained with the FB-protocol but, given the large errors reported by Yamada et al, they are not inconsistent Yamada's $f$ value, however, agrees reasonably well with our BH-protocol $f$ value

There are several important deficiencies of the study reported by Yamada et al relative to our FB study These include 1) the scanner hardware, although possibly state-of-the-art when the experiments were performed in about 1998, is considerably inferior to the state-of-the-art equipment used for our study, 2) they only used $4 b$-values - this is not enough to properly characterize a noisy b1exponential decay, 3) they used a BH-protocol instead of a protocol with a NRT sequence and 4) they report that the DW images used in the study were degraded by chemical shift and motion artifacts Given these deficiencies and the large reported errors, the values reported by Yamada et al for the IVIM parameters are not considered to be reliable

\subsubsection{The IVIM study of Lucianı et al.}

Lucianı et al [9] investigated the IVIM parameters with a respiratory triggered technıque that uses a pneumatic belt to track the motion of the abdominal wall externally The MR sequence is triggered to start image data acquisition when a specific pressure is detected by the belt This is an outdated method for performing respiratory triggering that has largely been replaced by the use of navigator echoes at sites that have this capability Triggering with the pneumatic belt has three primary deficiencies relatıve to triggerıng with navigator echoes 1) 
it is less sensitive to motion, 2) tracking the motion of the diaphragm (using navigator echoes) gives better reproducibility for the positioning of the abdominal organs with normal breathing, 3) the pneumatic belt must be secured farly snugly around the patient's abdomen, 4) with the pneumatic belt the patient must be coached to breathe in a very regular fashion throughout the exam Consequently, there is a higher probability that the measurements performed for a given DW experiment will not all correspond to the same voxel when a pneumatic belt is used for respiratory triggering, navigator echo triggering is much less sensitive to all of these issues

For the imaging sequence that we used, a navigator echo signal is obtained from a ROI placed perpendicular to the diaphragm This signal is used to track the location of the diaphragm The measured diaphragm positions are then passed to a respiratory triggering algorithm Image acquisition is initiated when the diaphragm is at a prescribed location The image acquisition is done during the end of the expiration phase, and there is one trigger event per breathing cycle See Section 24 for further detalls

Lucianı et al obtained the value of $D$ by performing a linear fit to the high $b$-value part of the decay, assuming that this part of the decay follows a single exponential decay function The estımation of $f$ and $D^{*}$ was done using a nonlınear regression algorithm based on the Nelder-Mead Simplex method with the value for $D$ fixed to the previously determined value This simplex method often encounters problems with local minıma [81] The analysis was performed on a pixel-by-pixel basis to generate parametric maps for $f, D$ and $D^{*}$ ROIs were 
then positioned on these parametric maps and the mean values for the ROIs were reported Two ROIs were placed in the right lobe and one in the left lobe but, unfortunately, the authors do not specify where withın the right and left lobes these ROIs were located They observed no significant variation in the parameters between the right and left lobes The average ROI size was $12 \pm 3$ pixels with a range of 10 to 16 pixels They comment in their paper that it was difficult to determine where major vessels were on their parametric maps

Lucianı et al report a $D$ value of (1 $16 \pm 09) \mu \mathrm{m}^{2} / \mathrm{ms}$ for a group of 25 patients This is in good agreement with our values, averaged over 8 subjects, of $D=\left(\begin{array}{lll}1 & 10 \pm 0 & 16\end{array}\right) \mu \mathrm{m}^{2} / \mathrm{ms}$ for the WRL-ROI and $D=\left(\begin{array}{lll}1 & 06 \pm 022\end{array}\right) \mu \mathrm{m}^{2} / \mathrm{ms}$ for the PRL-ROI Although the mean $D$ values are in very good agreement, the precision 1s much higher in our study, thanks to the superior methodology employed The $\mathrm{CV}$ value for their study is 078 whereas it is only 015 for the WRL-ROI and 021 for the PRL-ROI data in our study - a very significant improvement Their $D^{*}$ values are considerably different from ours but, given their large quoted errors, they are not inconsistent Their $f$ value is significantly lower than ours (see $p$-values for significant statıstıcal differences in Table 36 for all three parameters investigated)

Although the approach used by Lucianı et al is much better than that used by Yamada et al there are still some important deficiencies that make their reported IVIM parameter values questionable The use of a pneumatic belt for respiratory triggering does not provide good reproducibility of abdominal organ positioning for the images, as described in more detall above Navigator echo 
triggered respiratory gatıng is a far better approach However, a bigger concern is the method they used for their analysis According to their paper they obtained their $D$ values by fitting the data for the highest $2 b$-values to a simple exponential decay In the absence of noise this approach should be perfectly valıd However, since they did a pixel-by-pixel analysis for signals from relatively small voxels $\left(20 \mathrm{~mm}^{3}\right)$, with a moderately large TE of $70 \mathrm{~ms}$ and only a small amount of signal averaging $(\mathrm{NEX}=3)$, the diffusion decays were likely very noısy Although they do not report the SNR for their DW images, they do show a typical decay for normal liver in their Fig 1 and it is clear that the data are indeed quite noisy This no doubt explains why they report such a large error for $D$ Thus, the values that they report for $D$ must be considered questionable By comparison, we generate our diffusion decay from the mean of at least 100 pixel values so that the SNR for our decay is much higher than theirs and we have 8 of these high SNR data points (for the FB-protocol) in the large $b$-value part of the decay Moreover, the questionable $D$ values that they report are used as input to the simplex algorithm used to get $f$ and $D^{*}$ so that the values reported for these parameters are also suspect

It may appear contradictory to claim that our $D$-value is reliable but the value reported by Lucianı et al is suspect when the two values are very close ((1 $10 \pm 016) \mu \mathrm{m}^{2} / \mathrm{ms}$ and $(116 \pm 090) \mu \mathrm{m}^{2} / \mathrm{ms}$, respectıvely) However, we believe this agreement to be coincidental Given their large error, their $D$-value is compatible with values between $026 \mu \mathrm{m}^{2} / \mathrm{ms}$ and $206 \mu \mathrm{m}^{2} / \mathrm{ms}$, a huge range of values To investigate this further we transcribed the diffusion decay shown in 
Fig 1 of their paper as best we could and performed a bi-exponential fit to the data We obtained a very convincing fit with the parameters $f=046, D=073$ $\mu \mathrm{m}^{2} / \mathrm{ms}$ and $D^{*}=38 \mu \mathrm{m}^{2} / \mathrm{ms}$ This value for $D$ is very different from their quoted mean but compatıble with our BH-value and the value reported by Yamada et al The $f$ and $D^{*}$ values from this fit are also very different from the values reported by Luciani et al but they are in good agreement with the values that we report for the FB-protocol Although we only analysed one of their decays, and our approach was rather crude, the results can be taken as additional confirmation that the values reported by Lucianı et al are not reliable

Our data analysis procedure employed a more consistent approach in which 1) 3 equivalent 100-pixel ROIs were drawn peripherally (anterior, lateromedial, posterior areas of the right lobe of the liver) on anatomical images takıng care to avold regions containıng large vessels and 2) a large ROI was drawn to cover the whole liver We report values averaged over all slices for both the PRL-ROI and for the WRL-ROI Because the NRT-DW sequence used did not limit the imaging time or, as a consequence, the number of slices that could be acquired, it was possible for us to investigate the whole right lobe of the liver

The results obtained with the WRL-ROI and the 100-pixel ROIs revealed that there are no large deviations in the IVIM parameters for the areas of the liver that were investigated Even so, it should be recognized that, while part of the varıance for the experıments reported here is related to experımental error, part of it will also be due to small changes in the tissue from one area of the liver to another, as can be seen in the parametric maps, and from one subject to another 
Patel et al [95] reported in 2010 a prelıminary experience where IVIM and DCE are used alone and/or in combination to study its utility in diagnosing liver cirrhosis diagnosis In their study, the IVIM parameters were investigated with a similar technique to ours The data were acquired on a $15 \mathrm{~T}$ Siemens MR scanner and the diffusion protocol is similar to our imaging protocol as well However, the image analysis is different, segmented bi-exponentıal fittıng being used in their approach Even though the fitting procedures and statistical analysis tools used were different in the two studies, the values that we obtained for the IVIM parameters were similar to theirs, although our precision was at times better (see CV values in Table 37 )

\subsection{CONCLUSION}

By using a better imaging technique and a superior approach to the data analysis we were able to perform more accurate and precise IVIM parameter quantification compared with other studies reported in the literature $[9,12]$ We also showed that a free breathing protocol using a navigator echo respiratory triggered protocol is far superior to a breath hold protocol for measuring the IVIM parameters in the liver Moreover, this is the first study where a whole right lobe ROI was investigated and thus we can report, for the first tıme, average IVIM parameter values for the whole right hepatic lobe

The IVIM parameters discussed in this chapter were studied in four different ways 1) with an ROI that covered the whole right lobe of the liver (1 e the WRL-ROI), 2) with 3 separate square 100-pixel ROIs located around the 
periphery of the right lobe of the liver, 3) with an ROI that consisted of the union of the three 100-pixel ROIs (1 e the PRL-ROI) and 4) with a pixel-by-pixel analysis from which parametric maps were generated The parametric maps clearly showed changes in $f$ and $D^{*}$ between central and peripheral locations, consistent with blood flow patterns in the liver It was, therefore, expected that differences in the WRL-ROI and PRL-ROI IVIM parameter values would also be found While such a trend was suggested by the data, the effect was farrly subtle and could not be resolved beyond experimental error It was, therefore, concluded that there is no advantage to carefully positioning several smaller ROIs to avold major blood vessels and that the use of a WRL-ROI 1s a better approach IVIM parameters evaluated with a navigator echo free breathing protocol have significant potential as both sensitive and specific parameters for the detection and diagnosis of hepatic disease The improved methodology and the accurate IVIM parameter values for normal liver tissue presented in this chapter both represent s1gnificant advancements and are an important first step toward the use of IVIM parameters in MRI studies of the liver The IVIM parametric maps, which showed good anatomical detall, also have considerable promise as useful visual diagnostic tools for radıologists 
Chapter 4

Using an IVIM Model to Assess Blood Flow in the Placenta 


\subsection{Introduction}

In this chapter, Diffusion Weighted (DW) MR measurements of human placenta in vlvo are presented The fetal-placental-uterine system has not been well investigated with MRI in the past due primarily to motion artifacts in images caused by fetal and/or maternal motion during the scans These artıfacts can now be largely avoided using advanced techniques on modern MR scanners with higher amplitude gradients, single shot echo planer imaging (EPI) sequences, respiratory triggerıng with navigator echoes (e g PACE, Siemens Medical Solutions, Erlangen, Germany and parallel imaging capabilities [44,45] The resulting images have superior image quality with 1mproved SNR and they are not degraded by motion artıfacts The introduction of parallel imaging techniques and navigator echoes to track the motion of the diaphragm for respiratory triggering are the most important recent advancements for abdominal MR imaging, in general, and for placental imaging in particular The imaging protocols used to acquire the images reported in this thesis take advantage of these advances

The work presented here demonstrates the ability of MRI to 1) make high quality non-invasive in vivo measurements of human placenta using the intravoxel incoherent motion (IVIM) method [76] and 2) accurately evaluate the diffusion and pseudo-diffusion coefficients and the perfusion fraction, collectively known as the IVIM parameters A better understanding and increased accuracy of these parameters for the placenta, as presented in this chapter, are very important for the evaluation of patients with a suspected placental pathology where the IVIM parameters can be expected to be different from those in healthy subjects An 
example of such a pathology is fetal growth restriction (FGR) where the fetus is abnormally small because the placenta is not providing to the fetus the nutrition it needs for proper growth It should be noted that none of the subjects studied in the work presented here had FGR

\subsection{Materials and Methods}

\subsubsection{Subject Population}

This study was approved by The Ottawa Hospital Research Ethics Board Written informed consent was obtained from all participants Ten volunteers, ranging in age from 23 to 37 years old, were recruited The Gestational ages of the fetuses ranged from 18 to 35 weeks One of the volunteers was not scanned due to claustrophobia The first volunteer was scanned prior to a major upgrade on the scanner using a different protocol ( $1 \mathrm{e}$ a breath hold protocol) and the results for this subject will not be included here The analysis presented in this chapter is thus for 8 subjects Enrolment in the study was done by Drs Gruselin and Morett1, obstetricians working at The Ottawa Hospital Everyone involved in the study, other than the obstetricians, was blinded to the health of the subjects At the tıme of imaging, all volunteers were considered to have a normal placenta Three patients had complications with their pregnancies which were confirmed by ultrasound or at birth None of these complications were caused by placental pathology Information about the health of the subjects was not avallable to the researchers until after the analysis presented in this thesis was completed 


\subsubsection{MR technique}

MR imaging was performed at the General Campus of The Ottawa Hospital using a 15 Tesla (T) MR system (5Magnetom Symphony® Quantum TIM, Siemens AG, Erlangen, Germany) with a maxımum gradient strength of $30 \mathrm{mT} / \mathrm{m}$ All MR images were obtained with a multi-channel phased array torso matrix coil positioned anteriorly and elements of the spine matrix coil posteriorly The hospital's routine clinical pelvic MR protocol was used with all subjects as part of the experimental protocol An example of an image of a typical healthy placenta can be seen in Fig 41 The total imaging time for all sequences in the protocol was about $8 \mathrm{~min}$ per patient

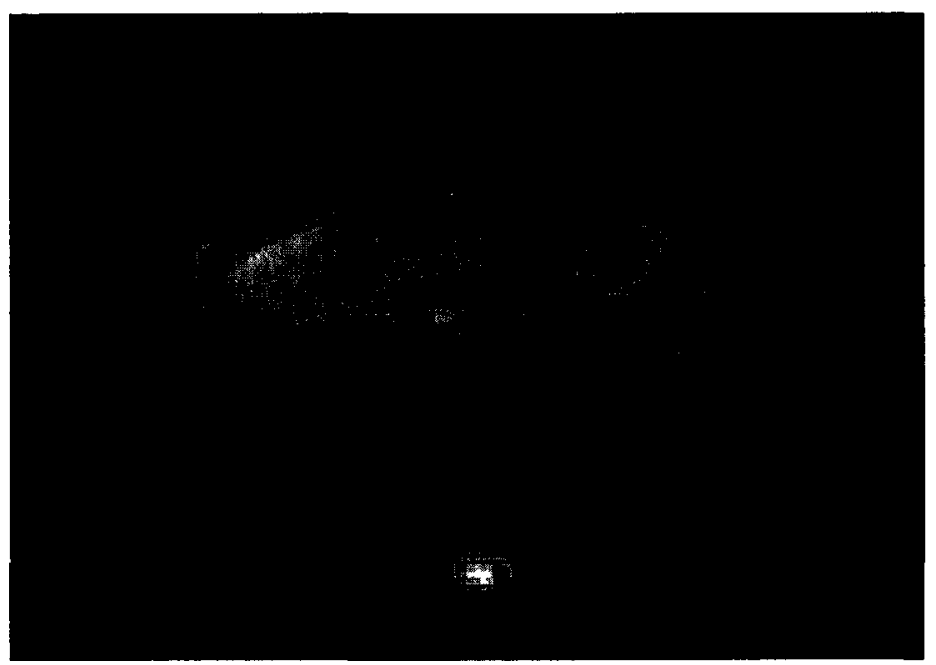

Fig. 4.1. MRI 1mage showing the anatomy of a healthy placenta (slice 19, patient 2) This image was acquired with a diffusion weighted echo planar sequence with $b=0$ 
Diffusion Weighted Imaging (DWI) of the entire placenta was performed using a DW single shot (SS) echo planar imaging (EPI) sequence with 2D-PACE (see section 24 ), a navigator echo respıratory triggering (NRT) technıque (1 e a sequence called ep2d_diff_p2_trigg was used), with small variations in the protocol from subject to subject TR ranged from 307958 to $586583 \mathrm{~ms}$ due to the patients' different breathing cycles, $\mathrm{TE}=94 \mathrm{~ms}$, eighteen $10 \mathrm{~mm}$ thıck axıal slices were acquired with a $25 \mathrm{~mm}$ inter-slice gap, the FOV was $400 \mathrm{~mm}$, phase FOV $=75 \%$, matrix size $=144 \times 192$, in-plane resolution $=21 \times 21 \mathrm{~mm}^{2}$ and the IPAT-GRAPPA reduction factor $=2$ An acquisition tıme of $2 \mathrm{~min} 4 \mathrm{~s}$ was required for the DW sequences to acquire all slices and all $b$-values When the training period for the 2D-PACE module is included the total acquisition time for the DW imaging was between 3 and 4 minutes, depending on the respiratory cycle of the patient For the experiments reported here, the acceptance window on the diaphragm location was set to $\pm 2 \mathrm{~mm}$ for triggering the image acquisition part of the sequence Fat suppression was used to suppress chemical shift artıfacts The fat suppression was done using the SPAIR technique which uses a combination of spectral fat saturation with a short time inversion recovery STIR sequence to suppress the signal from fat

The diffusion weightıng was performed using a dual echo pulsed gradient spin echo (PGSE) module with eddy current compensation [82] in front of the EPI echo train (see section 2312 ) Diffusion gradients were applied in the slice selection direction ( $1 \mathrm{e}$ in the head-to-foot direction) with the gradient pulse duration, $\delta$, and separation, $\Delta$, set to $45 \mathrm{~ms}$ and $50 \mathrm{~ms}$, respectıvely Only one 
direction was chosen due to time constraints on the scanner and in consideration for the comfort of the pregnant volunteers For each slice, 13 DW images were obtained, one for each diffusion gradient amplitude applied The $b$-values used in the protocol were $0,50,100,150,200,250,300,350,400,450,500,650$ and 800 $\mathrm{s} / \mathrm{mm}^{2}$ The values for the signal to noise ratio (SNR) for $b=0 \mathrm{~s} / \mathrm{mm}^{2}$ and $b=800$ $\mathrm{s} / \mathrm{mm}^{2}$ were approxımately 291 and 51 , respectıvely

\subsubsection{Image Analysis}

The decay of the DW MR signal versus $b$ is normally considered to be exponential in tissues, however, a b1-exponential signal decay versus $b$ was assumed in the presented study due to the presence of incoherent pseudo-random motion other than diffusion in the tissue Blood flowing in the microvasculature of a tissue causes the signal attenuation to be larger than that caused by diffusion alone and the effect is observed as a pseudo-diffusion process This interpretation 1s known as intravoxel incoherent motion (IVIM) theory [76] Accordingly, the signal attenuation curves reported in this thesis were analysed using IVIM theory and the decays were assumed to obey the equation (see also section 233 )

$$
S I / S I_{0}=(1-f) \exp (-b D)+f \exp \left(-b D^{*}\right)
$$

where $S I$ is the measured signal intensity, $S I_{0}$ is the measured signal intensity with no diffusion gradient applied, $b$ is the gradient factor of the pulse sequence (1 $\mathrm{e}$ the " $b$-value"), $D$ and $D^{*}$ are the true diffusion coefficient and the pseudodiffusion (or perfusion) coefficient, respectively, and $f$ is the fractional volume of 
the voxel occupied by spins in the microvasculature of the tissue ( $1 \mathrm{e}$ the perfusion fraction)

The diffusion decays were analysed using a region of interest (ROI) approach with the ROIs chosen in several different ways Initially, three rectangular 100-pixel ROIs were sampled for each slice of the placenta (see Fig 42) These ROIs were carefully positioned to avoid areas of fast turbulent flow where the blood spurts into the placenta from the spiral arteries of the mother In a separate analysis, a larger ROI was drawn to encompass as much of the placenta as possible for each slice (see Fig 42) This will be referred to as the whole placenta ROI or the WP-ROI For all four of these ROIs, the mean and standard deviation (SD) of the pixel intensities were computed for each $b$-value and all slices These mean values were then used to generate the diffusion decays The decays for all ROIs were distinctly b1-exponential (see Fig 43 ) and were analyzed usıng a Levenberg-Marquardt nonlınear least-squares fit to Eq 41 to obtain values for $D, f$, and $D^{*}$ The values plotted in the graphs and reported in the tables represent these parameter values for each ROI averaged over all slices for each volunteer

$D, f$ and $D^{*}$ maps were also calculated for the WP-ROI on a pixel-by-pixel basis for each slice The decays were again clearly bi-exponentıal and were analysed using the IVIM model The IVIM parameter values obtained from this analysis were used to generate parametric maps (see Fig 4 4) which were overlaid in colour onto the corresponding anatomical images ( 1 e the $b=0 \mathrm{~s} / \mathrm{mm}^{2}$ images) 
In a further analysis, the placenta was subdivided, using the anatomical ımages, into two approxımately equal ROIs (see Fig 42) termed ROI out, corresponding to the outer part of the placenta, towards the uterine wall, and $\mathrm{ROI}_{\mathrm{in}}$, corresponding to the inner area closest to the fetus [57] These two ROIs were drawn "by hand" for each slice, using IDL's ROI drawing tool, such that they had approxımately equal areas with their common side defining the bisector of the placental cross-sectional area The mean value for each of these ROIs was calculated for each $b$-value and diffusion decays were generated from these mean values for each slice The fit was performed as explaned above for the 100-pixel ROIs and the IVIM parameter values were obtaned for each ROI and each slice The difference between the values obtained for $\mathrm{ROI}_{\text {out }}$ and $\mathrm{ROI}_{\mathrm{in}}$ was then calculated for each of the IVIM parameters for every slice in each patient The difference values reported below were averaged across all slices for each subject

All image analysis was performed using software written in the MRI lab at The Ottawa Hospital using IDL (ITT Visual Information Solutions, Boulder, CO) 


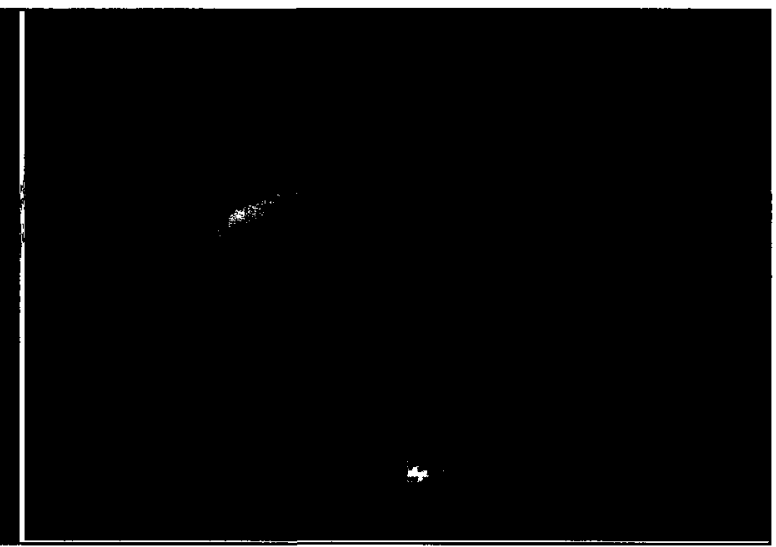

Fig. 4.2. The WP-ROI (red) and the three 100-pixel rectangular ROIs (blue) are shown on the image on the left and the ROIs drawn around the outer (red) and inner (blue) parts of the placenta are shown on the image on the right

\subsection{Results}

DW images were obtained for 8 volunteers No motion artifacts were detected in the images and fetal motion did not preclude analysis for any of the women scanned No significant change in the position of the fetus or the placenta, from the beginning to the end of the study, was noted for any of the subjects The 1mages did not require rectified noise bias correction since the SNR for the highest $b$-values used did not drop below 51 All diffusion decays, whether they were for individual pixels or ROIs, were clearly bi-exponentıal (see Fig 4 3) 


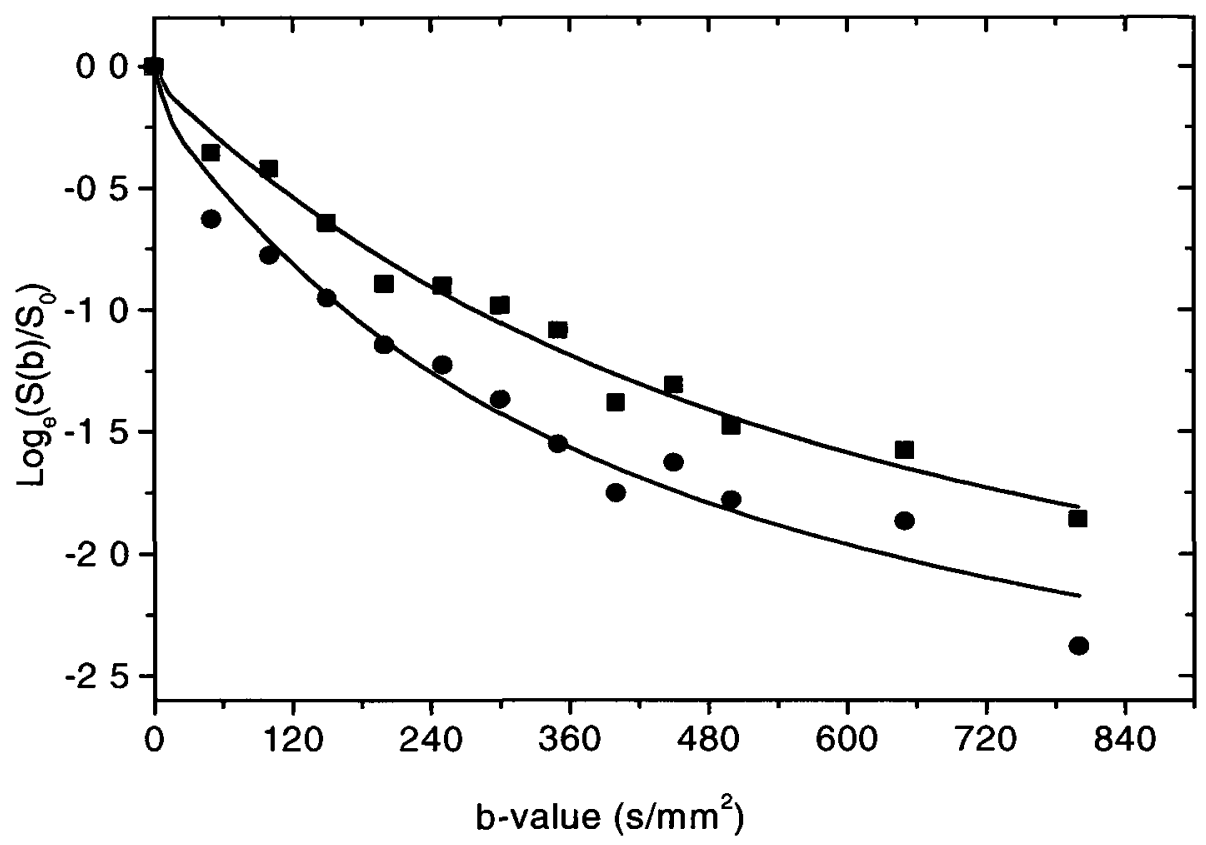

Fig. 4.3. Typical diffusion decays for the WP-ROI and a 100 pixel-ROI The decays shown are for the WP-ROI (black) and for one of the 100 pixel ROIs (red) shown in (slice 19, patient 2)

\subsubsection{IVIM Parametric Maps}

In Fig 44 color parametric maps of $f, D$ and $D^{*}$ for a healthy pregnancy are shown overlaid onto the corresponding anatomical image Inner and outer zones can be seen more clearly on the IVIM parameter maps than on the raw images Variations of this trend were obtained for the three patients with abnormal conditions

\subsubsection{IVIM Parameters for Different ROIs}

Values for $D, f$, and $D^{*}$ were obtained for 1) three rectangular 100-pixel ROIs and 2) for the WP-ROI The mean, computed as the average over all slices and all 
pixels contained in the union of the three 100-pixel ROIs, 1s also shown in the figures and is labelled as U-ROI This data sets are presented in Figs 45 to 410 Figures 45,47 and 49 show the variation of $f, D$ and $D^{*}$, respectively, for the three 100-pixel ROIs averaged over all slices The parameter values for each of these ROIs are all withın one SD of each other for each subject showing that the intra-subject agreement is good for all three IVIM parameters It also
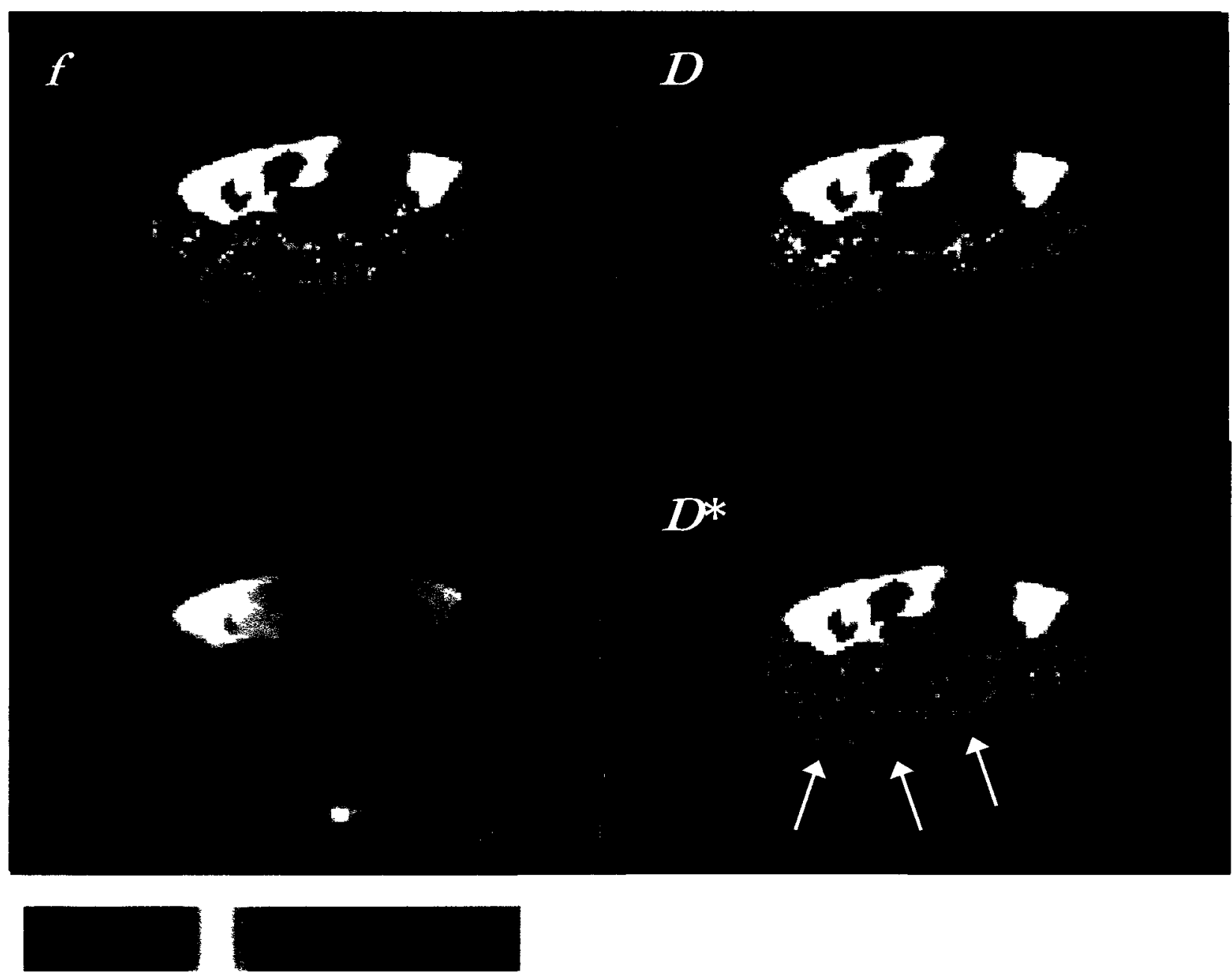

Fig. 4.4. An anatomical image as well as $D, D^{*}$ and $f$-maps, color overlaid onto an anatomical image, are shown for a healthy pregnancy (slice 19, patient 2) Two areas of blood movement can clearly be seen on the $D^{*}$-map (shades of blue and green, respectively) and the $f$-map Areas where maternal blood enters the placenta from the spiral arteries can be seen and are indicated by the arrows on the $D^{*}$ map The color table ranges for the maps are a) $0-100 \%$ for the $f$-map, b) $(0-2) \mu \mathrm{m}^{2} / \mathrm{ms}$ for the $D$-map and c) $(0-200) \mu \mathrm{m}^{2} / \mathrm{ms}$ for the $D^{*}$-map 
shows that intra-slice variations are small for these ROIs which contain approximately equal volumes of placenta from the fetal and maternal sides Figures 46,48 and 410 show the variation of $f, D$ and $D^{*}$, respectively, for the WP-ROI and the U-ROI, averaged over all slices in both cases

The variation of the IVIM parameters with slice position was also considered No consistent systematic changes were observed with slice position for any of the subjects or ROIs considered Figs 411 to 413 show the variation of the IVIM parameters with slice position for a typical data set For some of the subjects there was an indication of a small inter-slice variation for $f$ and $D^{*}$ but this trend could not be discerned beyond experimental error Therefore, the values in all of the tables and figures (except Figs 411 to 4 13) of this chapter are averaged over all slices

No statistically significant difference was observed between the IVIM parameter values for the WP-ROI and the U-ROIs, however, a trend towards higher values for the WP-ROI data was noted For $D$, the WP-ROI value was higher than the U-ROI value in all cases but this difference was not statistically significant since the $D$ values for these two ROIs were within one SD of each other for all subjects (see Fig 4 8) A similar observation was made for $f$ except that, in this case, two of the WP-ROI values are slightly more than one SD higher than the corresponding U-ROI value (see subjects 7 and 8 in Fig 4 6) This trend can also be seen in Fig 410 where the WP-ROI value for $D^{*}$ is higher than the U-ROI value for $D^{*}$ for all subjects except for subject 9 where the values are 
equal, in about half the cases the difference between the WP-ROI $D^{*}$ value and the U-ROI $D^{*}$ value is approximately equal to one SD

The results of the WP-ROI and U-ROI analyses suggest that the IVIM parameters do not vary across the placenta, either withın the slice or across slices For $D$, this observation is also supported by the $D$-map for the WP-ROI (see Fig 44) However, for the $f$ and $D^{*}$ parametric maps a variation from the maternal to the fetal side of the placenta is clearly seen This will be addressed further in sections 434 and 444 where ROIs from the fetal and maternal sides of the placenta are considered 


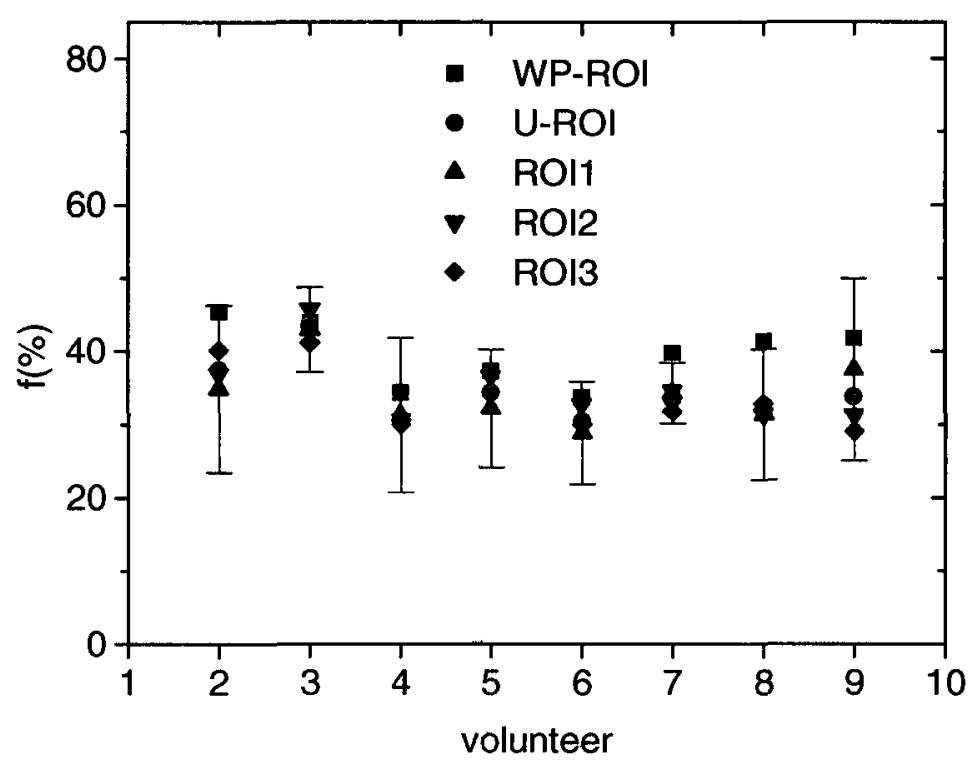

Fig. 4.5. Perfusion fraction, $f(\%)$, values plotted for each volunteer for 1 ) the 100-pixel ROIs, 2) the whole placenta ROI (WP-ROI) and 3) the combined 100pixel ROIs (U-ROI), averaged over all slices in each case For clarity, error bars are shown for ROI1 only The error bars correspond to the mean \pm SD

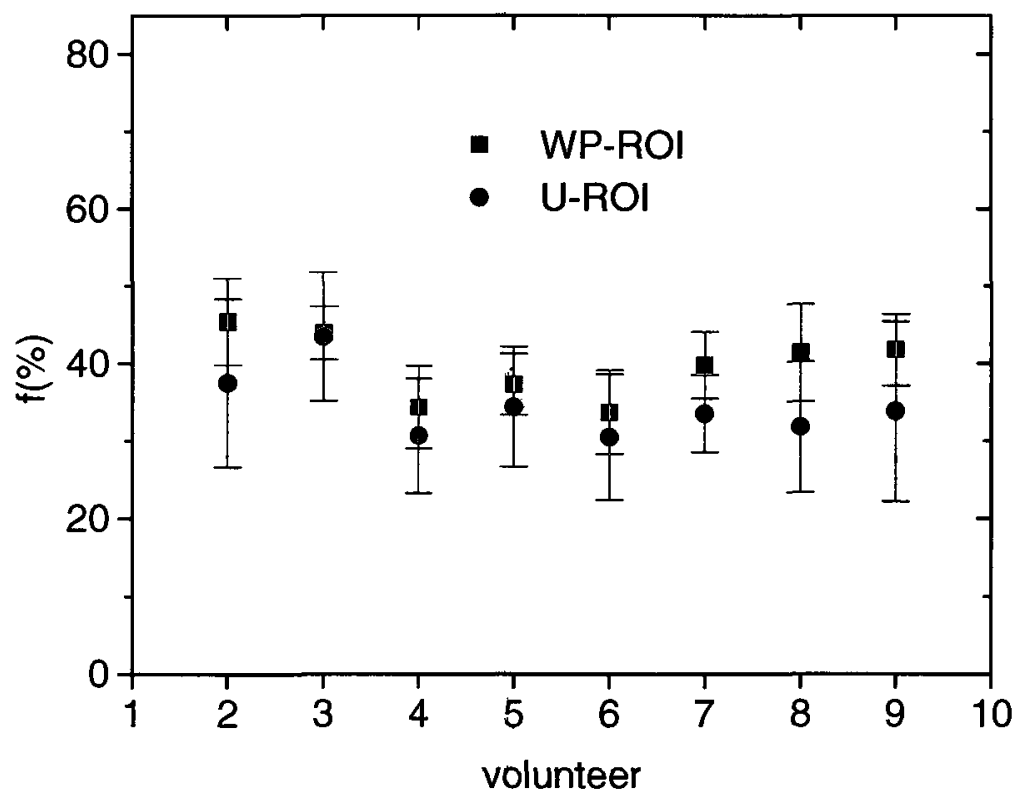

Fig. 4.6. $f$ values for each volunteer for the WP-ROI and the U-ROI, averaged over all slices in each case The error bars correspond to the mean \pm SD 


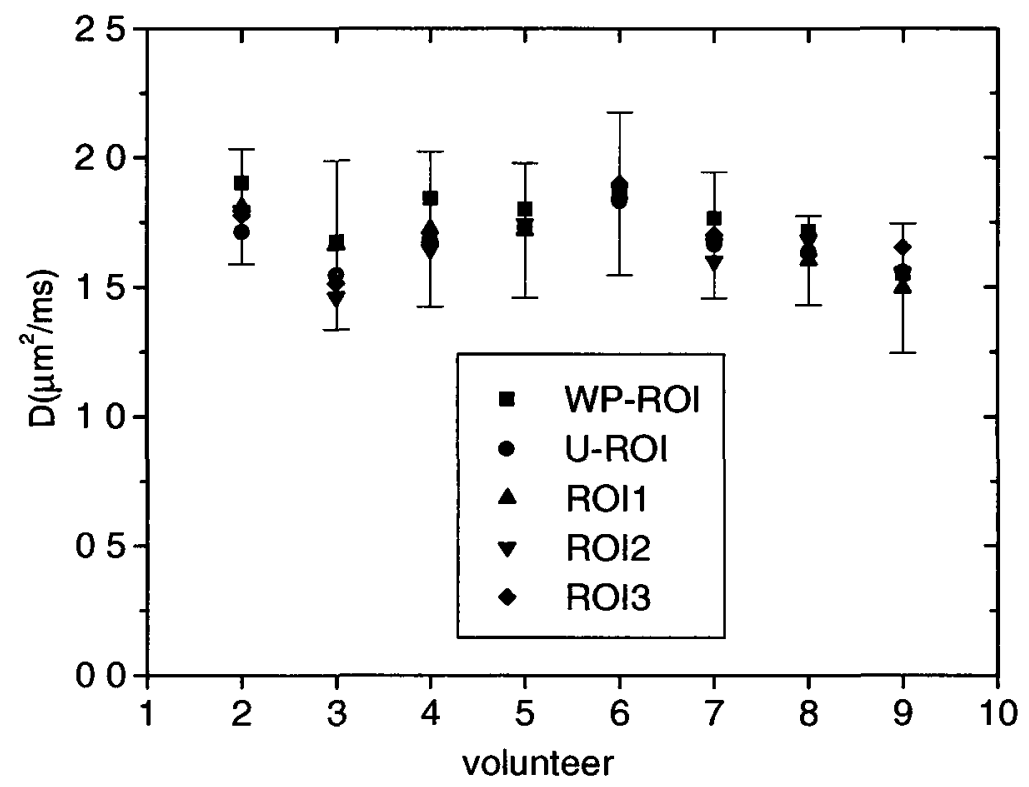

Fig. 4.7. Diffusion coefficient values plotted for each volunteer for 1) the 100pixel ROIs, 2) the WP-ROI and 3) the U-ROI, averaged over all slices in each case For clanty, error bars are shown for ROI1 only The error bars correspond to the mean $\pm \mathrm{SD}$

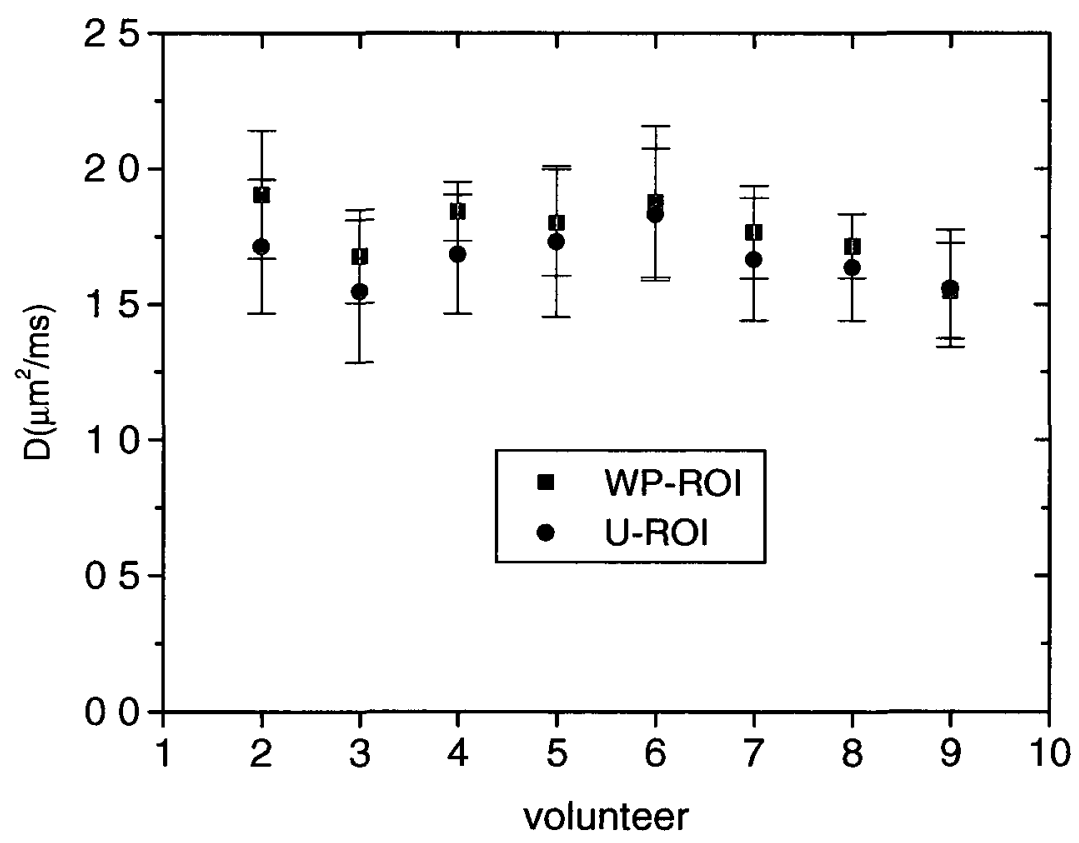

Fig. 4.8. $D$ values plotted for each volunteer for the WP-ROI and the U-ROI, averaged over all slices in each case The error bars correspond to the mean \pm SD 


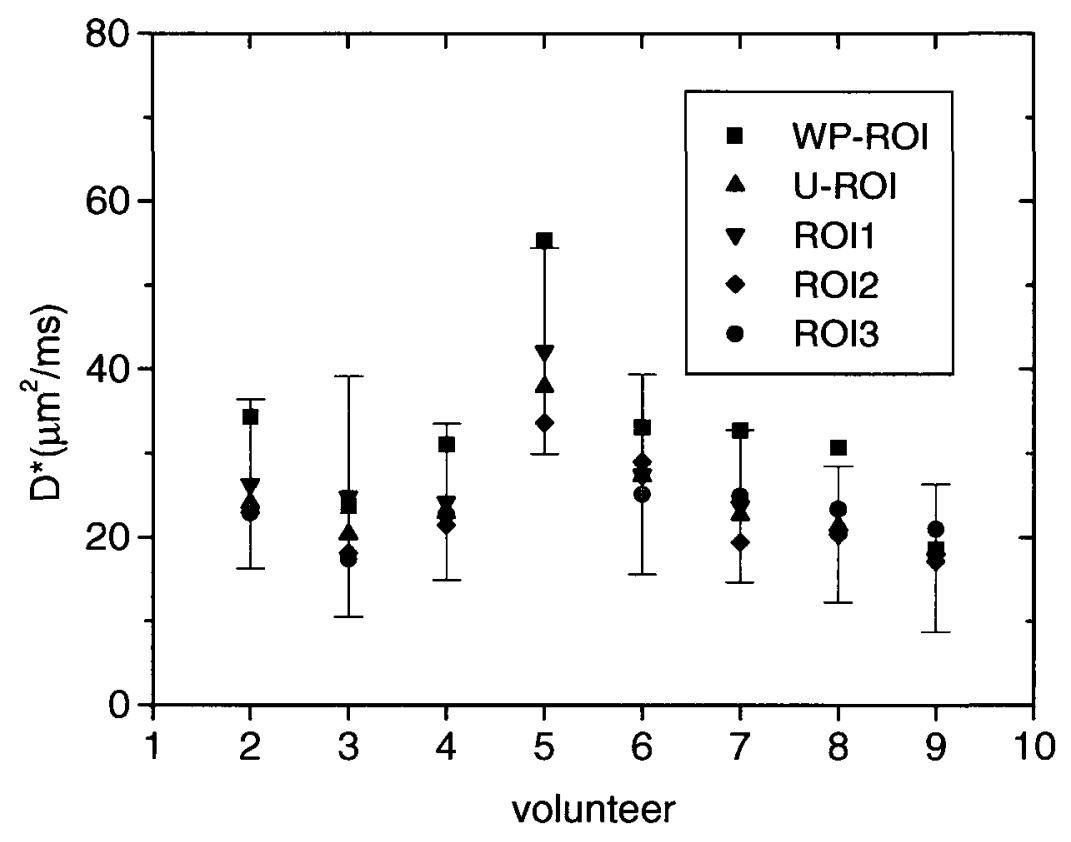

Fig. 4.9. Pseudo-diffusion coefficient values plotted for each volunteer for 1) the 100-pixel ROIs, 2) the WP-ROI and 3) the U-ROI, averaged over all slices in each case For clanty, error bars are shown for ROI1 only The error bars correspond to the mean $\pm \mathrm{SD}$

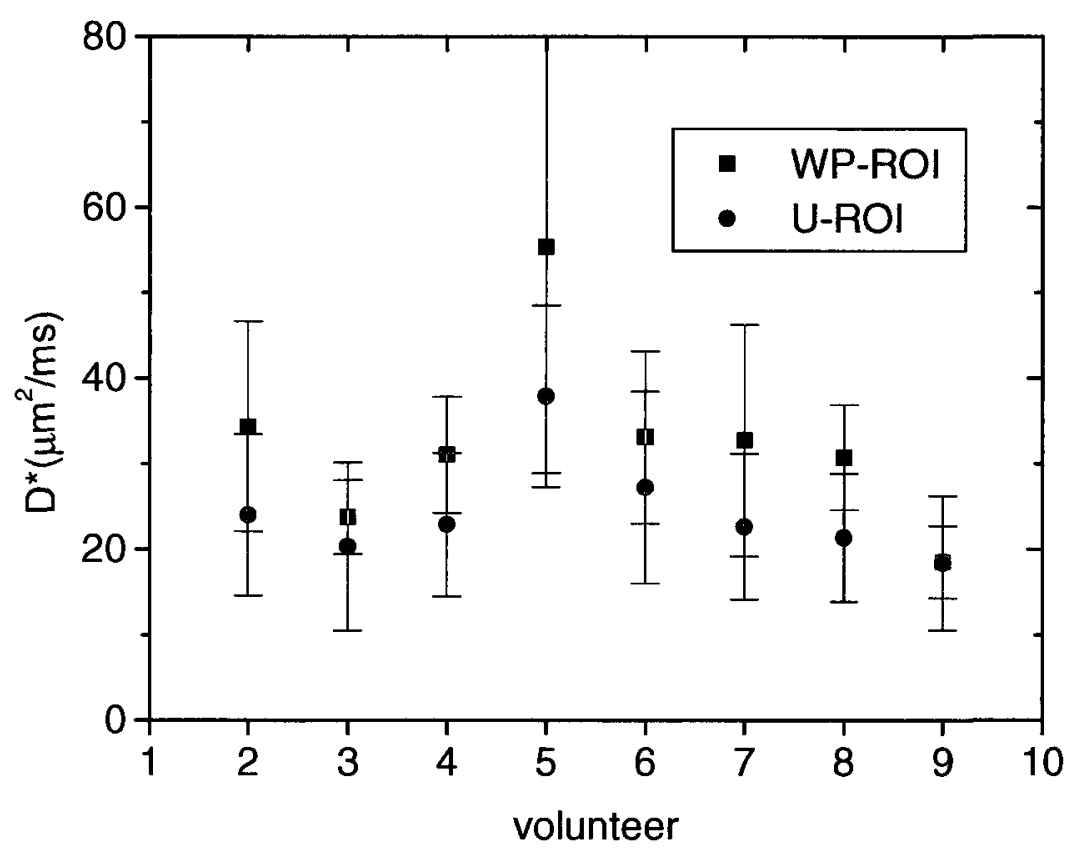

Fig. 4.10. $D^{*}$ values plotted for each volunteer for the WP-ROI and the U-ROI, averaged over all slices in each case The error bars correspond to the mean \pm $\mathrm{SD}$ 


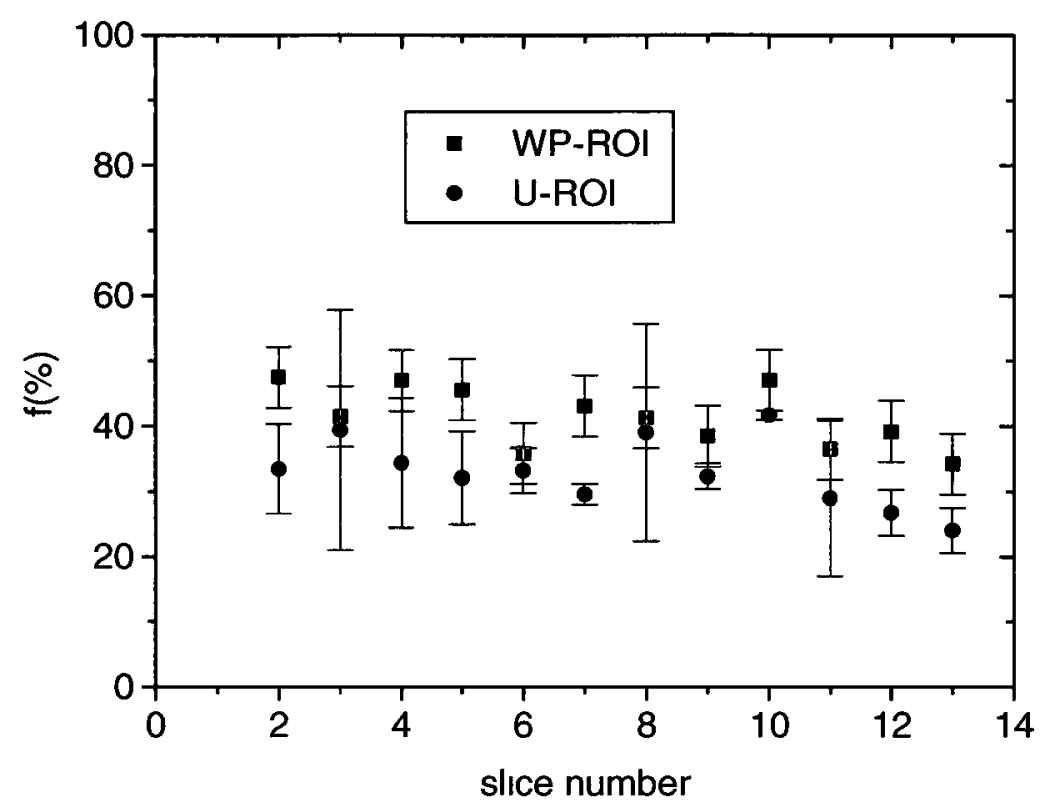

Fig. 4.11. Typical behaviour for $f$ plotted as a function of slice number for the WP-ROI and the U-ROI The data are from patient 9 The error bars correspond to the mean $\pm \mathrm{SD}$

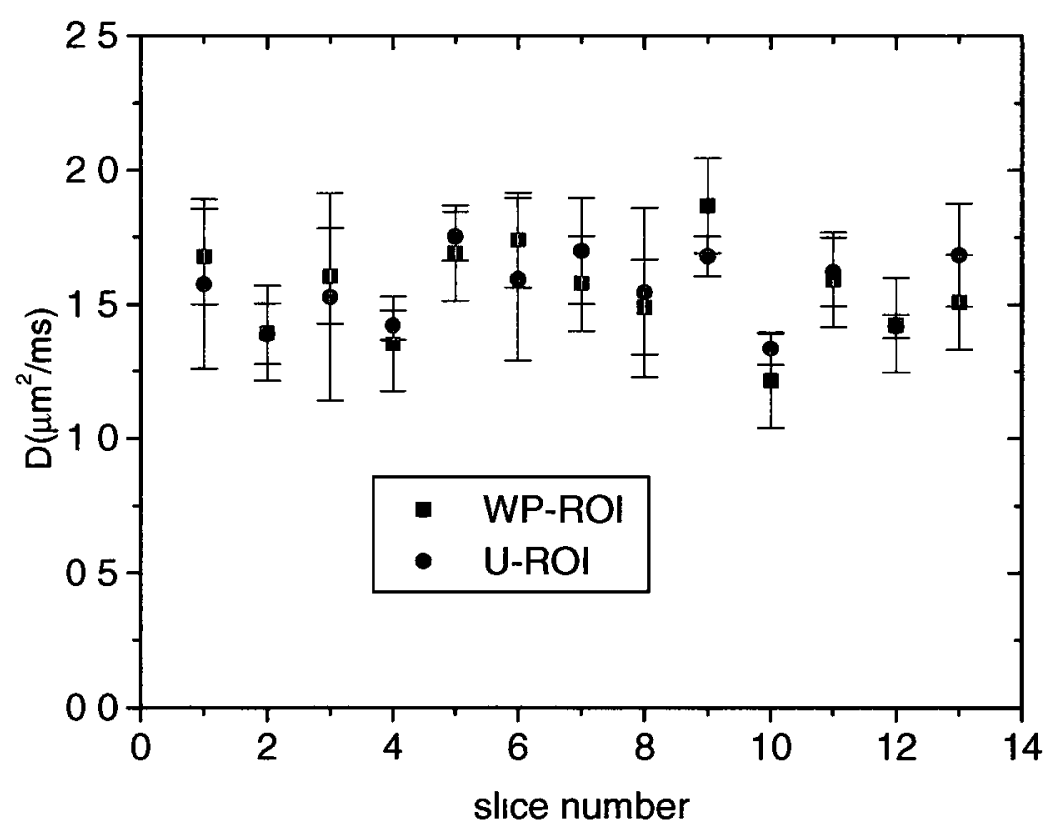

Fig. 4.12. Typical behaviour for $D$ plotted as a function of slice number for the WP-ROI and the U-ROI The data are from patient 9 The error bars correspond to the mean $\pm \mathrm{SD}$ 


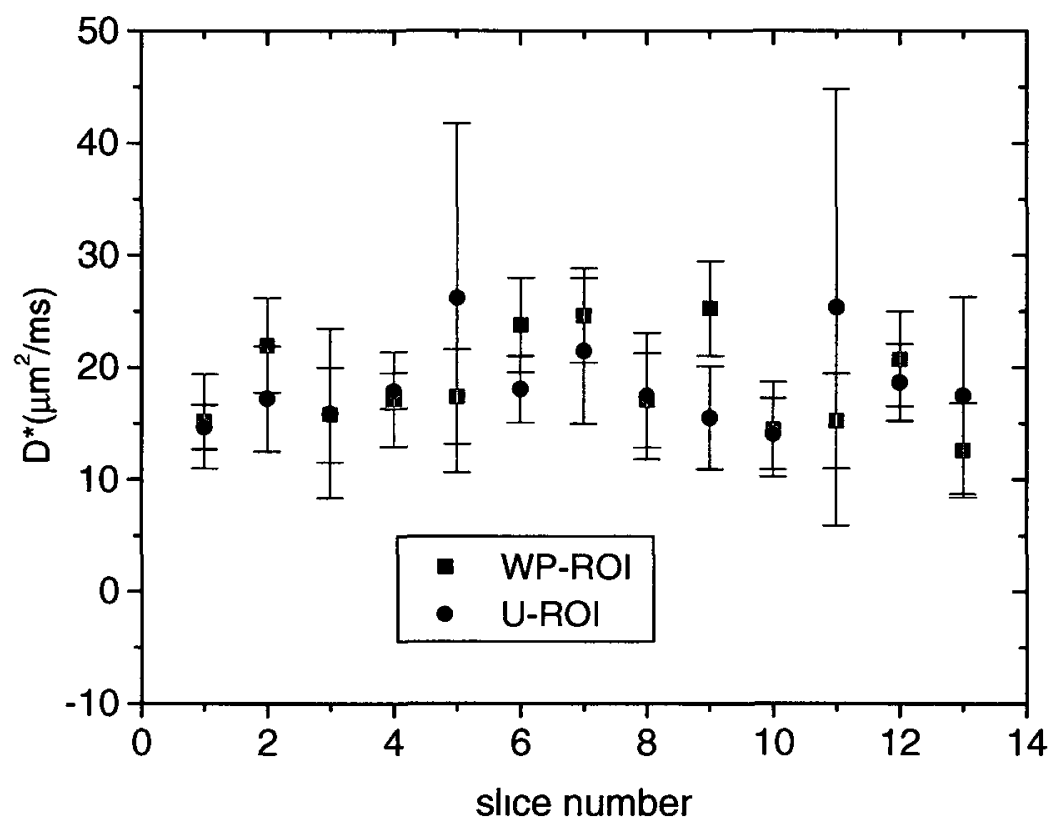

Fig. 4.13. Typical behaviour for $D^{*}$ plotted as a function of slice number for the WP-ROI and the U-ROI The data are from patient 9 The error bars correspond to the mean $\pm \mathrm{SD}$

\subsubsection{IVIM Parameters Versus Gestational Age}

To determine if the IVIM parameters change durnng the pregnancy, they were also plotted as a function of gestational age (GA) (Figs 414 to 4 16) A linear least squares fit was performed on these data for all three IVIM parameters for both the WP-ROI and the U-ROI The resulting slope values are given in Table 41 From a visual inspection of the figures there appears to be a subtle change in some of the parameters with GA, however, these apparent variatıons are not statıstıcally significant Thus, to within experimental error, all of the IVIM parameters are constant for GA $=18$ to 35 weeks and we report the IVIM parameter values in Table 42 as the mean over GA for this time period 


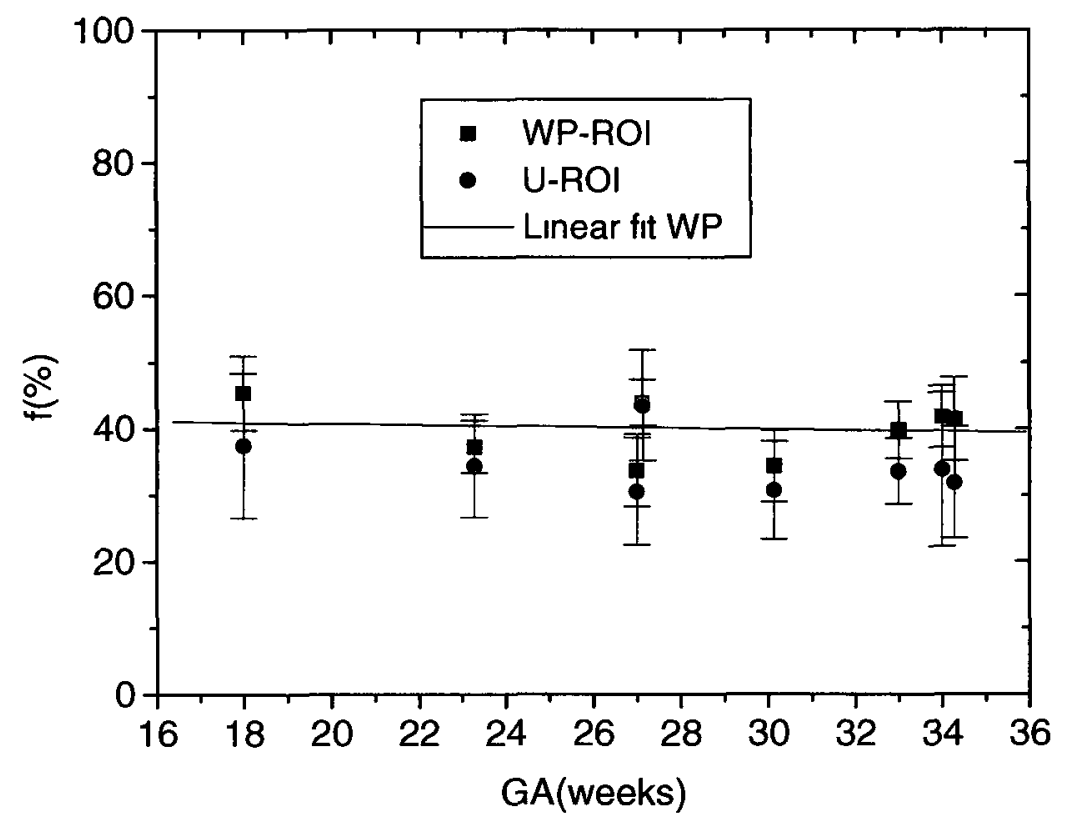

Fig. 4.14. $f$-values for the WP-ROI and the U-ROI plotted as a function of gestational age (GA) The error bars correspond to the mean \pm SD The black line shows the linear least squares fit to the WP-ROI data

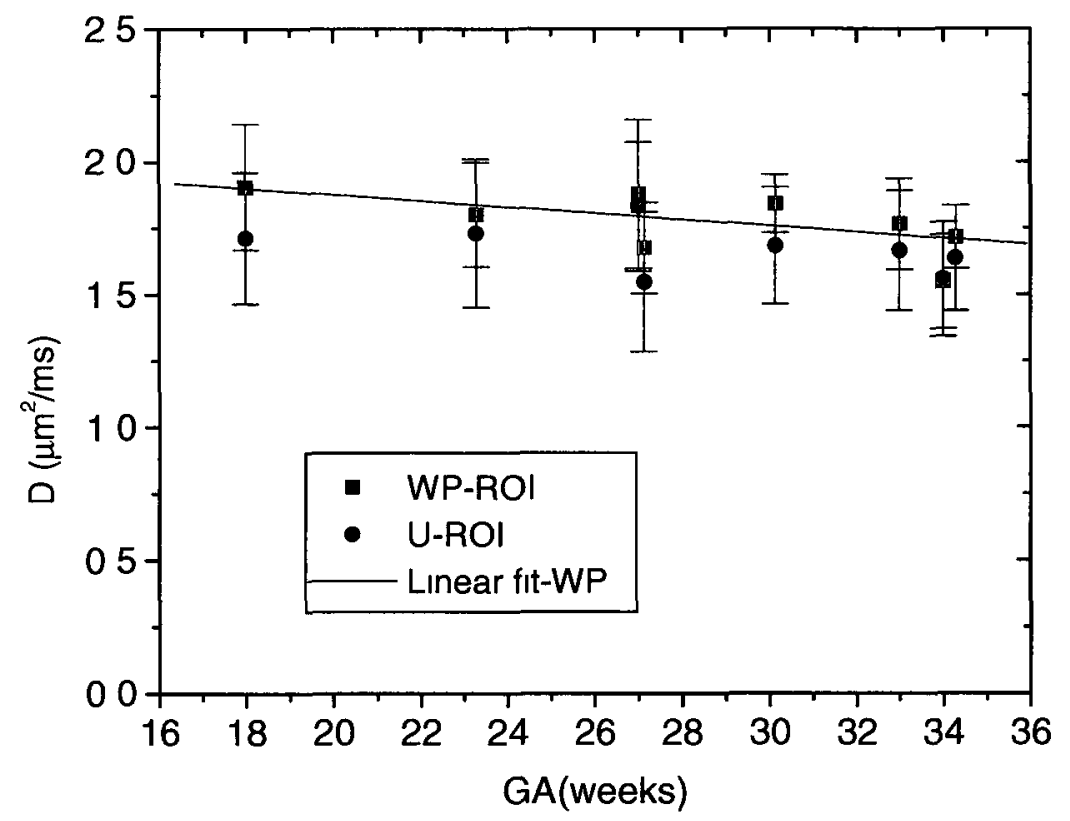

Fig. 4.15. $D$-values for the WP-ROI and the U-ROI plotted as a function of gestational age (GA) The error bars correspond to the mean \pm SD The black line shows the linear least squares fit to the WP-ROI data 


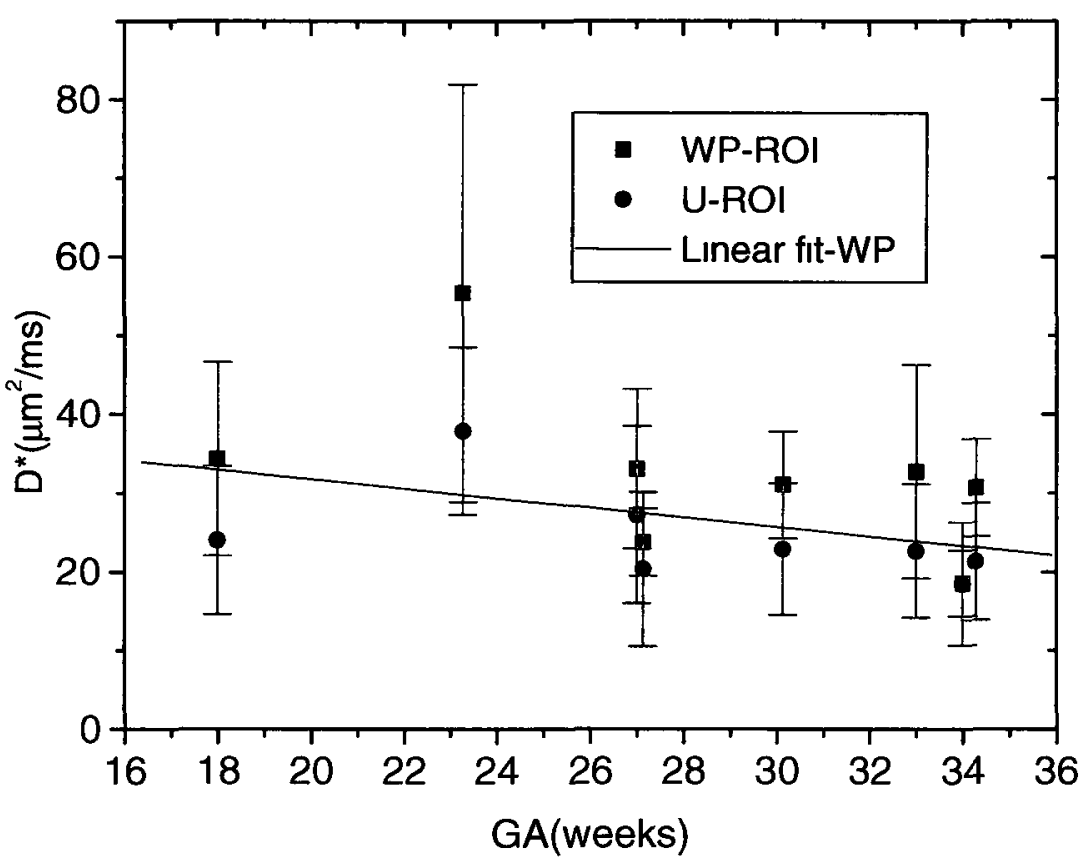

Fig. 4.16. $D^{*}$-values for the WP-ROI and the U-ROI plotted as a function of gestational age $(\mathrm{GA})$ The error bars correspond to the mean $\pm \mathrm{SD}$ The black line shows the linear least squares fit to the WP-ROI data

Table 4.1. Slopes for the IVIM parameters vs GA

$$
f(\% / \mathrm{wk}) \quad D\left(\mu \mathrm{m}^{2} \mathrm{~ms} / \mathrm{wk}\right) \quad D^{*}\left(\mu \mathrm{m}^{2} / \mathrm{ms} / \mathrm{wk}\right)
$$

WP-ROI $-01 \pm 03 \quad-001 \pm 001 \quad-06 \pm 06$

U-ROI $\quad-03 \pm 03 \quad-001 \pm 001 \quad-02 \pm 02$

Table 4.2. Mean IVIM parameter values averaged over $\mathrm{GA}=18$ to 35 weeks

$$
f(\%) \quad D\left(\mu \mathrm{m}^{2} / \mathrm{ms}\right) \quad D^{*}\left(\mu \mathrm{m}^{2} / \mathrm{ms}\right)
$$

WP-ROI

$40 \pm 6 \quad 18 \pm 02 \quad 30 \pm 12$

U-ROI

$$
34 \pm 6 \quad 17 \pm 03 \quad 23 \pm 10
$$

p-value (t-test, two tailed) $\quad 0065 \quad 0446 \quad 0225$

Note Data are reported as mean \pm SD Mean parameters were obtaned by averaging the fit parameters over all subjects for both the WP-ROI and the U-ROI (if $p$-value $<005$, then results are statıstıcally different) 
Table 43 Mean IVIM parameter values averaged over GA $=18$ to 35 weeks (Ottawa) and the Notthingam data, when a $t$-test was performed, and a two tailed $p$-value is reported

\begin{tabular}{cccccc}
\hline $\begin{array}{c}\text { IVIM } \\
\text { Parameter }\end{array}$ & $\begin{array}{c}\text { Notthıngam } \\
(\mathrm{N}=11)\end{array}$ & $\begin{array}{c}\text { WP-ROI } \\
(\mathrm{N}=8)\end{array}$ & $\begin{array}{c}p \text {-value } \\
(\text { Notthıngam } \\
\text { vs WP-ROI })\end{array}$ & $\begin{array}{c}\text { U-ROI } \\
(\mathrm{N}=3)\end{array}$ & $\begin{array}{c}p \text {-value } \\
\text { (Notthıngam vs } \\
\text { U-ROI })\end{array}$ \\
\hline$D\left(\mu \mathrm{m}^{2} / \mathrm{ms}\right)$ & $17 \pm 05$ & $18 \pm 02$ & 06103 & $17 \pm 03$ & 0999 \\
$D^{*}\left(\mu \mathrm{m}^{2} / \mathrm{ms}\right)$ & $57 \pm 41$ & $30 \pm 12$ & 00905 & $23 \pm 10$ & 00358 \\
$f(\%)$ & $26 \pm 6$ & $40 \pm 6$ & 00001 & $34 \pm 6$ & 00106 \\
\hline
\end{tabular}

Note Data are reported as mean \pm SD (If $p$-value $<005$, then results are statıstıcally different)

All statistical analyses were done using ROIs SPSS software was used for statistical computations All ROI distributions were assumed to be normal for these initial analyses and therefore the means and standard deviations of the data were reported A $t$-test was used to compare the means between WP-ROI and UROI as well as with values reported in the literature Not having the literature data in our possession, we could not be sure of the form of the distributions but we made the assumption that their data are also normally distributed Mean comparıson tests were performed and a two sided asymptotically significant $p$ value was reported in all cases Statistical significance was defined as $p<005$ However, the assumption of normality was re-examined in a secondary analysis by calculatıng the skewness and kurtosis for the data sets and by performing a Shapiro-Wilk test of normality With this secondary analysis it was found that the $D^{*}$ parameter distributions did not obey the condition of normality and a MannWhitney non-parametric test was therefore used in these cases These results are found in Appendix B 


\subsubsection{IVIM Parameters for $\mathrm{ROI}_{\mathrm{in}}$ and $\mathrm{ROI}_{\text {out }}$}

The parametric maps shown in Fig 44 suggest that there may be a difference in the IVIM parameters between the fetal and maternal sides of the placenta (see section 2 5) ROIs were defined to contain these zones, as described earlier, and the IVIM parameters for these ROIs were determined Figure 417 shows typical b1-exponential signal decays for both the inner and outer areas of the placenta outlined in Fig 42 The values of the IVIM parameters for $\mathrm{ROI}_{\mathrm{in}}$ and $\mathrm{ROI}_{\text {out }}$ averaged over all subjects are summarized in Table 43

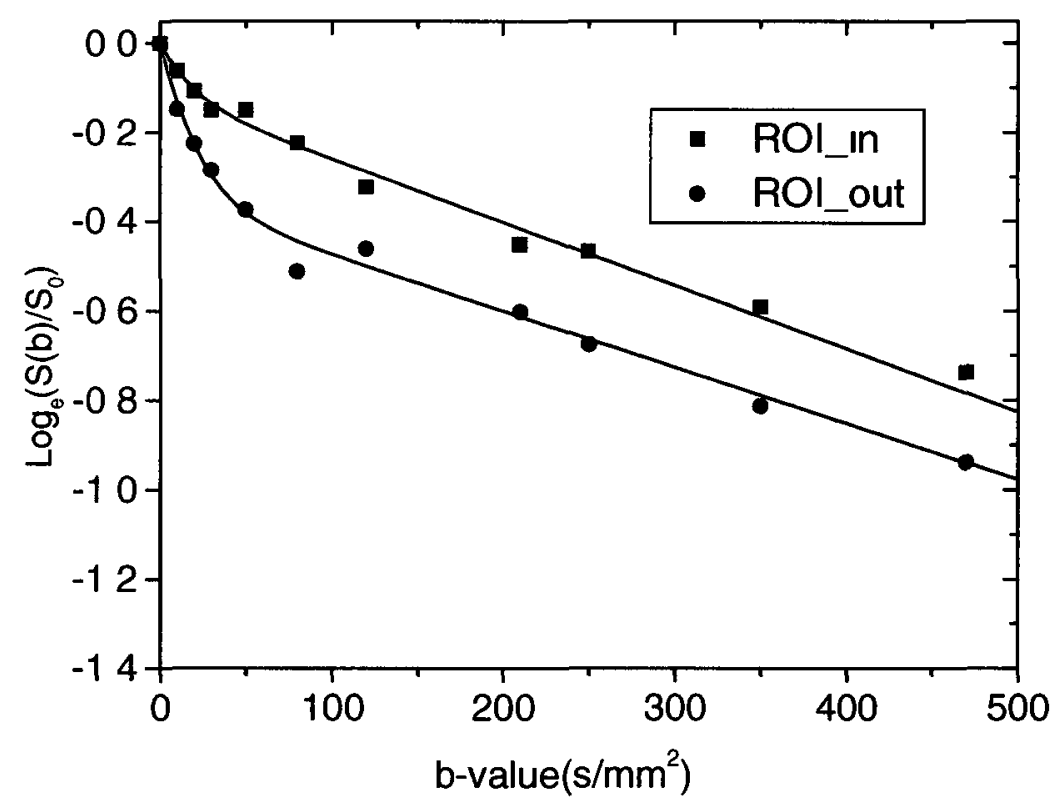

Fig. 4.17. Signal attenuation versus $b$-value for inner ( $1 \mathrm{e}$ fetal side) and outer ( $1 \mathrm{e}$ maternal side) placental regions for subject 1 , acquired using a breath hold technique The bi-exponential nature of the decays can clearly be seen 
Table 4.4. Mean IVIM parameter values averaged over all subjects

$$
f(\%) \quad D\left(\mu \mathrm{m}^{2} / \mathrm{ms}\right) \quad D^{*}\left(\mu \mathrm{m}^{2} / \mathrm{ms}\right)
$$

ROI In $\quad 38 \pm 8 \quad 17 \pm 04 \quad 29 \pm 20$

ROI $_{\text {out }} \quad 43 \pm 11 \quad 17 \pm 03 \quad 34 \pm 18$

Note Data are reported as mean $\pm \mathrm{SD}$

While $D$ shows similar values for the two areas, as expected, $f$ and $D^{*}$ do not The value of $f_{\text {out }}-f_{\text {In }}$ was positive for 5 of the 8 subjects investigated (see Fig 4 18) and $D_{\text {out }}-D_{\text {tn }}$ and $D_{\text {out }}^{*}-D_{\text {tn }}^{*}$ were both positive for 7 of the 8 patients in the study (see Figs 419 and 4 20)

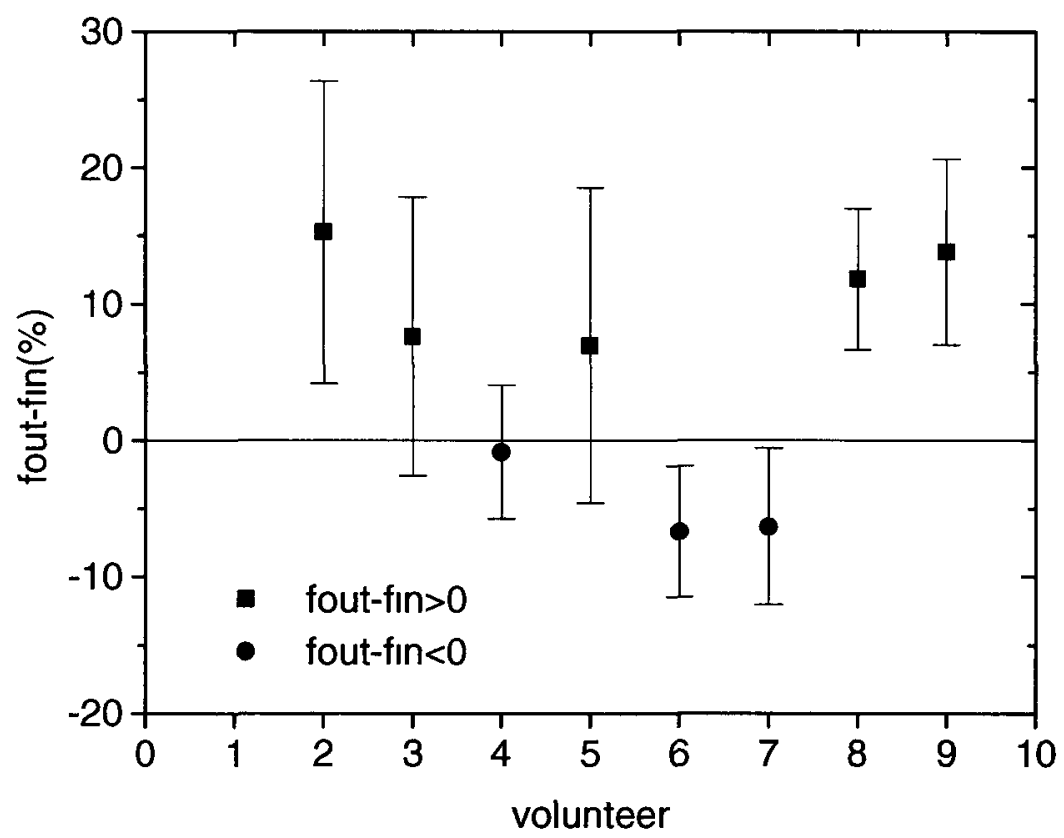

Fig. 4.18 $f_{\text {out }}-f_{\text {in }}$ values for each volunteer (mean $\pm \mathrm{SD}$ ) $f_{\text {out }}=f_{\text {in }}$ is indicated by a solıd black line and negatıve values are shown in red 


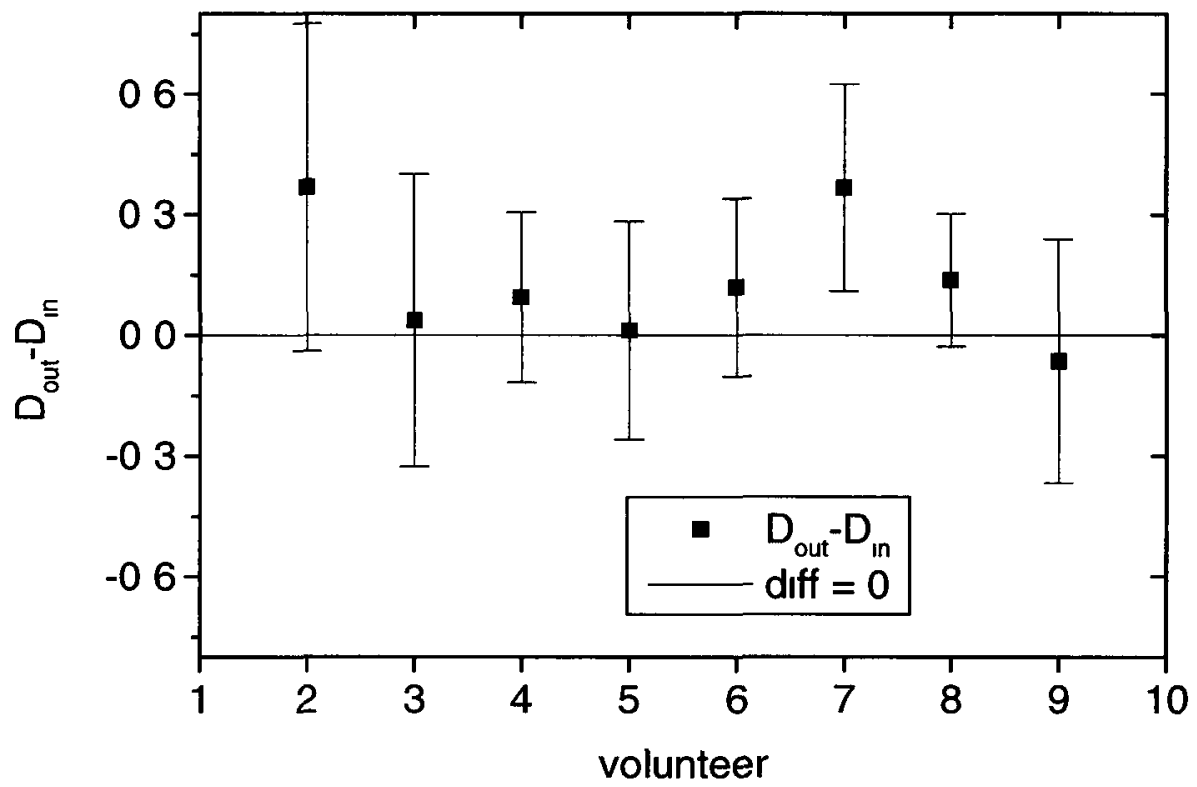

Fig. 4.19 $D_{\text {out }}-D_{\text {tn }}$ values for each volunteer (mean $\pm \mathrm{SD}$ ) $D_{\text {out }}=D_{\text {tn }}$ is indicated by a solid red line

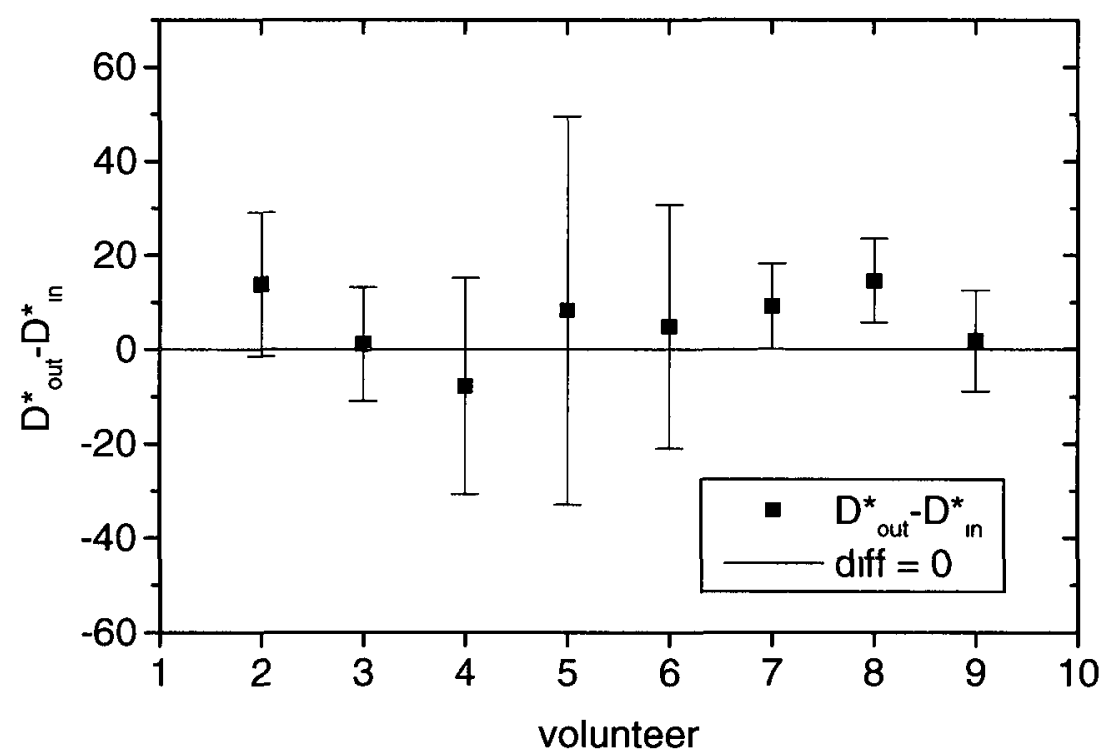

Fig. 4.20 $D_{\text {out }}^{*}-D_{\text {in }}^{*}$ values for each volunteer (mean $\pm \mathrm{SD}$ ) $D_{\text {out }}^{*}=D_{\text {in }}^{*}$ is indicated by a solıd red line 


\subsection{Discussion}

In this chapter, an MRI study of blood flow in and around the placental villi is presented Since it seemed reasonable to expect that the motion of the water molecules in the blood of the placenta can be explained in terms of a pseudorandom walk behaviour when viewed macroscopically, a DW MR sequence was used to track this motion The measured bi-exponential signal decays were analysed using the IVIM model (see section 233 for a review of the IVIM model) and an assessment of the fittıng parameters $f, D$ and $D^{*}$ was made The faster decaying component was associated with the slowly flowing blood in the microvasculature and the intervillous spaces of the placenta while the other component was assigned to a standard diffusion process for soft tissue (see section 25 for a review of placental anatomy and physiology)

The voxel size for the study presented here was $21 \times 21 \times 10 \mathrm{~mm}^{3}$, which is very large compared to the size of the fetal blood vessels of the placental villi The villı are highly branched structures with many small segments pointing in all directions [79] Thus, in a typical voxel of the placenta, a large anisotropic distribution of velocities for the fetal microcirculation can be expected For this reason, it would have been useful to study the behaviour as a function of the diffusion sensitization direction but, due to time constraints (as noted above) this was not done The maternal blood in the placenta enters the intervillous space from the spiral arteries in the uterine wall The intervillous space is a chamberlike area where the villı are bathed in nutrient rich blood from the mother The maternal blood in the placenta does not flow through small vessels in the placenta 
like the fetal blood does Instead, it forms a pool of blood that enters the intervillous space from the spiral arteries and leaves via the endometrial veins [79] The maternal blood entering the intervillous spaces spurts from the spiral arteries and, at this entry point, the flow is both unidirectional and fast Nevertheless, the flow of most of the maternal blood inside the intervillous space, which is deflected many times by the branches of the villi, is much slower and anisotropic As with the fetal blood, the maternal blood circulation can, therefore, be viewed as a random walk process On the scale of a voxel there is a large anisotropic distribution of velocities coming from both the maternal and fetal microcirculation This results in a contribution to the IVIM parameters from both maternal placental circulation as well as the fetoplacental microcirculation

In the last few years, new techniques proposed to improve the image quality and precision of diffusion measurements in abdominal imaging include parallel ımaging, respiratory triggerıng using navigator echoes and improved fat suppression All of these techniques were exploited for the measurements reported here Parallel ımaging is a relatively new technıque for MR signal acquisition that takes advantage of the multiple coll elements in state-of-the-art phased array rf receiver coils This technology can be used to reduce the acquisition time or to increase the number of measurements performed in the same time or a combination of the two The use of parallel imaging in combination with modern phased array coils leads to a considerable improvement in image quality 
Navigator echo Respiratory Triggered (NRT) sequences allow measurements to be made while the subject is breathing freely as opposed to holding her breath On a Siemens scanner the NRT procedure is known as 2DPACE (see section 24 for an explanation of 2D-PACE) The navigator echo MR signal that is acquired as part of the NRT technique is used to track the motion of the diaphragm and image acquisition occurs only when the diaphragm is at the desired position For the experiments reported here the acceptance window on the diaphragm location was set to $\pm 2 \mathrm{~mm}$ This helps to ensure that the measurements are done with the abdominal organs in the same location each time Other respiratory gating methods exist but they do not work as well and they require the use of an external device (e $\mathrm{g}$ a bellows or a pneumatic belt) placed on or around the patient's abdomen to track the motion of the abdominal wall throughout the respiratory cycle With the NRT technique no additional hardware is required This makes patient set up considerably easier and avoids patient discomfort related to the use of an external motion tracking device Furthermore, with NRT technique, the acquisition time is not limited to a single breath hold (15 $-20 \mathrm{~s}$ ) which would constrain the number of slices that can be imaged and affect the overall accuracy of the results obtaned The use of NRT for DW measurements in the abdomen is far superior to elther using a breath hold protocol or using other forms of respiratory triggering These considerations were discussed in some detall in Chapter 3 where similar measurements are reported for the liver 
Another factor that can have a significant negatıve effect on image quality with abdominal imaging is chemical shift artifacts Because the resonant frequencies of fat and water molecules are not the same, the position of adipose (1 e fatty) tissues is shifted in the images relative to other tissues For most MR sequences, this shift is only a few pixels and is not a major problem However, for EPI sequences, such as the ones used in this study, the shift can be a significant portion of the FOV With abdominal imaging using an EPI sequence, this often causes a bright "ghost" image of the subcutaneous adipose tissue to appear in the middle of the image, possibly obscuring parts of the image that are of specific interest An effective remedy for this is to suppress the signal from the subcutaneous fat However, traditional fat suppression techniques do not always work perfectly, with the result that the artifact is not removed completely The SPAIR technique used here is a combination of two quite different methods of fat suppression, spectral fat saturation and STIR, and does a much better job of completely suppressing the fat signal than previously available methods Chemical shift artıfacts were not a problem for any of the images in this study

Motion artıfacts caused by motion of the abdominal wall durıng the scan can also be a problem with abdominal imaging Motion artıfacts appear as displaced ghost images superimposed onto the image The major contribution to these artıfacts is often from the bright signal from subcutaneous fat so suppression of the fat signal with SPAIR not only removes chemical shift artıfacts but also the primary component of motion artifacts The use of 2D-PACE also minimizes the 
effect of motion artifacts Motion artifacts were not a problem for any of the 1mages in this study

The use of superior hardware with parallel imaging, superior fat suppression and NRT capabilities in our study made possible the acquisition of higher SNR 1mages and more slices with faster data acquisition speeds and overall improved image quality compared with previously reported placental perfusion studies These studies will be reviewed and compared to our work in some detall in the next section The images in our study show good anatomical detall and were not affected by maternal or fetal movement Using a high speed imaging technique and a free breathing protocol also reduces patient discomfort, an 1mportant consideration for pregnant women The DW-SS-EPI sequence used for these measurements has eddy current compensation incorporated into the design of the DW part of the sequence [82] so artifacts caused by eddy currents are also negligible The free breathing technıque used provides the flexibility of including multiple $b$-values in the protocol without limiting the number of slices investigated Thus, we were able to image the whole placenta and report IVIM parameter values averaged over the whole placental volume For each patient, 18 slices were acquired and between 8 and 14 of these slices contained placental areas

From the analysis point of view, great care was taken when positioning the square 100-pixel ROIs to avold areas where maternal blood spurts into the placenta since at these locations the fast flow strongly de-phases the magnetization causing the MR signal for these voxels to be very low 
Furthermore, the flow in these locations is not diffusion-like and it is not characteristic of the flow in the rest of the placenta From a practical point of view, inclusion of these fast flow regions was undesirable because it sometimes caused difficulties with the convergence of the bi-exponential fitting routines, partıcularly for the pixel-by-pixel calculations required to generate the parametric maps

\subsubsection{Placental IVIM Studies in the Literature}

To our knowledge there are only three other studies that have investigated the IVIM parameters for the placenta and all of them are from the same research group in Nottıngham The first two [56,57] were both reported in 2000 and were performed using the same hardware and experimental procedure For both, the experiments were done using a breath hold protocol on a lab-built $05 \mathrm{~T}$ scanner Although they did not mention which coil was used for signal detection it is likely that the built-in body transmit coil was also used as the receiver for both studies since this was a fairly common practice for abdominal imaging at the time these experiments were done and no other receiver coll was mentioned in either paper The sequence they used had a PGSE module for diffusion weightıng and an EPI module for image acquisition For the PGSE module they used a single spin echo with $\delta=184 \mathrm{~ms}$ and $\Delta=60 \mathrm{~ms}$ with no inherent eddy current compensation bult into the sequence The other reported parameter values, which were the same for both studies, are TE $=115 \mathrm{~ms}, \mathrm{TR}=10 \mathrm{~s}$, matrix $=128 \times 128$, 1n-plane resolution $=$

$25 \times 35 \mathrm{~mm}^{2}$, slice thıckness $=7 \mathrm{~mm}, \mathrm{SNR}=201$ and the total DW imaging tıme 
$=220 \mathrm{~s}$ They apparently measured only one axial slice They acquired $11 \mathrm{DW}$ Images with the following $b$-values $0,02,3,15,47,80,115,206,246,346$ and $468 \mathrm{~s} / \mathrm{mm}^{2}$ The DW gradient direction was not specified They did not use respiratory triggering or parallel imaging and there is no mention in their paper of using fat suppression

These papers differed in the subject population studied and in the ROI selection In the first paper [56], 11 healthy subjects were studied whereas in the second paper [57] 13 healthy subjects and 7 subjects with fetal growth restriction (FGR) were studied The analysis was done in the first paper using a single ROI of about 100 pixels positioned such that obvious artifacts and flowing blood were avolded whereas for the second study, ROIs were drawn around the inner and outer parts of the placenta, similar to the approach used here and 1llustrated in Fig 42 For both studies, a diffusion decay was constructed from the ROI means for each $b$-value and the IVIM parameters were determined by fittıng this decay to a bi-exponential function In the second study they were able to show, for a small number of patients with FGR, that the difference in perfusion fractions for these two ROIs was correlated with the presence of disease

These studies are good plot studies and, given the resources avallable at the time, were well done However, working at a lower field strength with less sensitive rf colls and a longer TE caused the SNR to be considerably lower for their study than ours Furthermore, since they did not use respiratory triggering, fat suppression or parallel imaging, their images would have been affected by chemical shift and motion artıfacts - as noted in their papers By sampling 
multiple areas of the placenta our IVIM parameter values are also more representative of the average value for the whole placenta and the variance due to experimental error is lower for our work The hardware and methodology used in our study are clearly superior and our IVIM parameters can therefore be considered to be more precise This is justified in more detall in the next section Comparison with their IVIM parameters must be done with caution since the accuracy of their reported values is uncertain

The Nottıngham group's third report dealıng with IVIM parameters for the placenta was a poster presented at the ISMRM conference in 2009 [58] For this work a 15 T Philıps Achieva scanner with a phased array body receive coll was used This study was better than their initial work because they used superior hardware, improved MRI parameter settıngs and a pseudo-respiratory-triggering protocol where the patients were asked to synchronize their breathing to the sound of the scanner gradients This pseudo-respiratory triggering protocol is superior to the breath hold technique used in their previous study but it is still quite subjective and requires a cooperative patient, there is no guarantee with this technique that the placenta will be in the same location for all measurements Their basic protocol appears to be similar to ours, however, we have introduced Important enhancements by using NRT and fat suppression

Even though this more recent study appears to be superior to the earlier ones, it is of little use to us since they do not report values for any of the IVIM parameters Instead they show histogram plots of the parameter distributions for the pixels in their ROI From a visual inspection of these distributions it can be 
deduced that the medians are consistent with the mean values reported in Table 42 but, since their distributions are very broad, and, in the case of $D^{*}$, quite skewed, any further quantıtatıve comparıson would be meaningless

In summary, there are only three reports in the literature of IVIM parameter measurements for the placenta For all of them, significantly inferior hardware and/or methodology were used compared with the work presented in this chapter None of these studies gives IVIM parameter values that are as reliable as those reported here To the best of our knowledge, there are no reports in the literature of IVIM parameters for the placenta that were measured using an NRT protocol

\subsubsection{Comparison of our IVIM Parameters with the Literature}

The intra-slice and inter-slice agreement for our results was very good for each of the 100-pixel ROIs and for the WP-ROI, for all three IVIM parameters The values obtained for the WP-ROI and the U-ROI were also in agreement to within experimental error, although there was a trend towards increased $f$ and $D^{*}$ values for the WP-ROI The inter-subject variability was also small for all parameters The data did not show a statıstıcally significant variation for any of the IVIM parameters as a function of GA for the range of gestational ages investigated in this study (1 e GA $=18-35$ wks) Thus, the IVIM parameter values reported here were averaged over all slices and all subjects

Eight pregnant volunteers were imaged and all were considered to have normal pregnancies at the time the MRI scanning was performed However, 
subsequently it was discovered that three of them had complications with their pregnancies patient 4 had low amnotic fluid volume, patient 6 had a dilated cervix and for patient 7 the placenta was twisted and stuck to the uterine wall of the eight volunteers enrolled in the study, only one required a premature delivery, but nether the baby nor the mother had any health related problems Additionally, medical assessment of the ultrasound for patient 5 showed that the patient was going into preterm labour None of these conditions relate directly to the health of the placenta They may, however, have had an indirect effect on the placental processes No cases of FGR were reported in our group of volunteers

\subsubsection{Diffusion Coefficient}

Only two of the studies in the literature report placental $D$ values The first one [56] gives $D=(17 \pm 05) \mu \mathrm{m}^{2} / \mathrm{ms}$ The other study [57] reported $D=(17 \pm 09)$ $\mu \mathrm{m}^{2} / \mathrm{ms}$ and $D=(14 \pm 08) \mu \mathrm{m}^{2} / \mathrm{ms}$ for the inner and outer parts of the placenta, respectively These values are all in very good agreement with our values of $D=$ (1 $8 \pm 02) \mu \mathrm{m}^{2} / \mathrm{ms}$ and $D=\left(\begin{array}{ll}17 \pm 03 \\ 1\end{array} \mu^{2} / \mathrm{ms}\right.$ for the WP-ROI and the U-ROI, respectıvely, and $D_{l n}=(17 \pm 04) \mu \mathrm{m}^{2} / \mathrm{ms}$ and $D_{\text {out }}=(17 \pm 03) \mu \mathrm{m}^{2} / \mathrm{ms}$ The results are not significantly different (see Table 43 ) The coefficients of variation (CV) for the three literature values given above are 029,053 and 057 , respectıvely, whereas our CVs are only $011,018,024$ and 018 for the WP-ROI, U-ROI, $\mathrm{ROI}_{\mathrm{in}}$ and $\mathrm{ROI}_{\text {out }}$ results, respectively Our largest $\mathrm{CV}$ value is for $\mathrm{ROI}_{\mathrm{in}}$ This may partly be caused by the more turbulent behaviour of the maternal blood in this ROI It should be noted that, although the mean $D$ values from the 
literature are in very good agreement with our values, the precision is much higher for our study, thanks to the superior methodology employed

\subsubsection{Pseudo-Diffusion Coefficient}

The value of $D^{*}$, averaged over all subjects, was found to be $D^{*}=(30 \pm 12)$ $\mu \mathrm{m}^{2} / \mathrm{ms}$ for the WP-ROI and $D^{*}=(23 \pm 10) \mu \mathrm{m}^{2} / \mathrm{ms}$ for the U-ROI The values that we report for $\mathrm{ROI}_{\mathrm{in}}$ and $\mathrm{ROI}_{\text {out }}$ are $D^{*}{ }_{\text {in }}=(29 \pm 20) \mu \mathrm{m}^{2} / \mathrm{ms}$ and $D^{*}{ }_{\text {out }}=(34 \pm$ 18) $\mu \mathrm{m}^{2} / \mathrm{ms}$ These values are all considerably lower than the previously reported value of $D^{*}=(57 \pm 41) \mu \mathrm{m}^{2} / \mathrm{ms}$ [56] but, given the large quoted error for the literature value, they are not inconsistent It should be noted that the precision for our values is much higher than for the literature value The CV for the literature study was 072 (56) whereas for our study it is only $040,043,069$ and 053 for the WP-ROI, the U-ROI, the $\mathrm{ROI}_{1 \mathrm{n}}$ and the $\mathrm{ROI}$ out, results, respectively This shows that the precision of our WP-ROI and U-ROI values is significantly better than for the value reported in the literature (see Table 43 ), due primarily to the superior methodology employed Values for $D^{*}{ }_{i n}$ and $D^{*}{ }_{o u t}$ have not yet been reported in the literature so a proper comparison cannot be made Our largest CV value is for $\mathrm{ROI}_{\mathrm{in}}$ This may partly be caused by turbulent behaviour of the maternal blood in this ROI

\subsubsection{Perfusion Fraction}

Only two of the studies in the literature report perfusion fraction values The first gives a value of $f=(26 \pm 6) \%$ with a reported trend towards a decrease of $06 \%$ 
per week [56] The second gives values of $(25 \pm 8) \%$ and $(35 \pm 11) \%$ for the inner and outer sections of the placenta, respectively (see Fig 4 2) [57] Our $f$ values are $(34 \pm 6) \%,(40 \pm 6) \%,(38 \pm 8)$ and $(43 \pm 11) \%$ for U-ROI, WP-ROI, ROI ${ }_{n}$ and $\mathrm{ROI}_{\text {out }}$ Although the ranges overlap in each case, the literature values are consistently lower than ours The precision of the $f$ value reported here is higher than for the values reported in the literature The CVs are 023,032 and 031 , respectively, for the studies in the literature whereas they are only $018,015,021$ and 026 , respectively, for our study

Although our $D$-value is in good agreement with the literature, the values of $f$ and $D^{*}$ reported here are somewhat different However, if the stated error ranges are considered, they are not statistically different We believe that our values are more precise since 1) we used a superior MR scanner that gives higher SNR and better image quality, 2) a superior imaging protocol, that included parallel ımaging, NRT and good fat suppression, was used, 3) the acquired images were essentially artifact free, 4) an improved approach to the data analysis was used and 5) we report values of the parameters averaged over all slices

\subsubsection{WP-ROI versus U-ROI}

It is anticipated that the blood dynamics will be different for different parts of the placenta In particular, for locations where maternal blood enters the placenta from the spiral arteries of the mother, the blood flow is expected to be relatively fast and somewhat turbulent with this behaviour diminishing with distance from the arterial input This was investigated by analysing ROIs that included and 
excluded the blood as it enters from the sprral arteries ( $1 \mathrm{e}$ the WP-ROI and UROI, respectively) Furthermore, it is expected that the blood dynamics on the maternal and fetal sides of the placenta will differ This was tested by comparing the IVIM parameters for $\mathrm{ROI}_{\mathrm{in}}$ and $\mathrm{ROI}_{\text {out }}$, ROIs drawn around the fetal and maternal parts of the placenta, respectively

The essential difference, in terms of blood dynamics, between the WPROI and the U-ROI is the presence in the WP-ROI of the relatively fast flowing, somewhat turbulent, blood from the maternal spiral arteries These regions were carefully excluded from the 100-pixel ROIs that constitute the U-ROI Based on this consideration, it is to be expected that both $f$ and $D^{*}$ will be higher in the WPROI than they are in the U-ROI but $D$ should be unaffected A trend can be seen in Fig 410 where the WP-ROI value for $D^{*}$ is higher than the U-ROI $D^{*}$-value for all subjects except subject 9 where the values are about equal, in approximately half the cases, the WP-ROI $D^{*}$-value is about one SD higher than the U-ROI $D^{*}$-value In contrast to this, the $D$-values obtained for the WP-ROI and the U-ROI are almost identıcal, as expected

\subsection{4 $\mathrm{ROI}_{\mathrm{in}}$ versus $\mathrm{ROI}_{\text {out }}$}

The IVIM parameters were also obtained for ROIs drawn around the inner and outer parts of the placenta These two areas can be distınguished in the anatomical images of the placenta and corresponding contrast was observed in the $f$ and $D^{*}$ parametric maps As expected, the $D$-values for these two regions were 
the same but the $f$ and $D^{*}$-values differed considerably This trend was also seen in the parametric maps (see Fig 4 4)

The parameters $f_{\text {out }}-f_{i n}$, the difference in the perfusion fractions and $D_{o u t}^{*}-D_{u n}^{*}$, the difference in the pseudo-diffusion coefficients attributed to the outer (maternal) and inner (fetal) areas of the placenta, respectively, were also considered It has been reported that the $f_{\text {out }}-f_{\text {in }}$ parameter is positive for healthy placenta [57] whereas $D_{\text {out }}^{*}-D_{\text {in }}^{*}$ values have not been reported previously For 5 of the 8 subjects in our study $f_{\text {out }}-f_{\text {in }}$ was positive and $D_{\text {out }}^{*}-D_{m}^{*}$ was positive for 7 of 8 subjects A negative value for the $f_{\text {out }}-f_{\text {in }}$ parameter was obtained for all 3 volunteers that had complications with their pregnancies as confirmed for each patient by the obstetrician based on ultrasound results One of these patients (volunteer 4) had negative values for both of these parameters Although these patients' conditions were not directly related to placental abnormalities it is quite conceivable that they could have had secondary effects on the blood dynamics within the placenta In our study, a positive value for $f_{\text {out }}-f_{\text {in }}$ was indicative of a healthy pregnancy in all cases and a negative value for either of these parameters was always associated with complications The negative value obtained for these parameters suggests the existence of an abnormal condition in this small preliminary study

The Nottıngham group associates the reduced $f_{\text {out }}-f_{\text {in }}$ value with FGR mainly and with compromised pregnancy, in general [57] (although their unhealthy group did not contain any patients other than FGR patients) We cannot make this prediction since none of the patients in our study had FGR or 
were considered to have had an abnormal placenta, they just presented with an abnormal condition in the pregnancy

\subsubsection{Coefficients of Variation for the IVIM Parameters}

In general, there will be two primary contributions to the observed variation in the measured parameters, one due to experimental error and the other due to changes in tissue composition across the ROI The IVIM parameters given by the Nottıngham group are averaged over a relatıvely small ROI from a single slice of the placenta whereas ours are averaged over larger intra-slice ROIs and over all slices of the placenta The fact that the CV values that we report are smaller than the literature values indicates that we had a smaller amount of experimental error and/or we more effectively removed the effects of random error by averaging over more of the placenta It also suggests that the inherent variation of the IVIM parameters across the placenta is small since, if this were not the case, our varıances would have been as large as or larger than those of the Nottıngham group, not smaller That being said, it is clear that part of the observed variation for $f$ and $D^{*}$ is due to changes in the tissue properties across the placenta since there are clear differences in these parameters, partıcularly $D^{*}$, for different parts of the placenta (see Fig 44)

The variation in $D^{*}$ was larger than for the other IVIM parameters and seems to be the parameter most affected by ROI location within the slice as well as slice position in the placenta A distribution of $D^{*}$ values is to be expected since $D^{*}$ will be higher for locations near where the blood spurts into the placenta 
from the spiral arteries and there will be a gradient of $D^{*}$ values as the distance from this area increases On the color $D^{*}$-maps (e g Fig 44 ), higher $D^{*}$ values are seen on the maternal side of the placenta where turbulent flow is expected, and a more constant behaviour is found for the rest of the placenta where the density of villi is very high Blood entering the placenta from the spiral arteries can also clearly be seen in these maps as increased $D^{*}$ values

The observed variation of $D^{*}$ may also be partly related to experımental factors The parameters $D$ and $f$ are determined by the fit to high $b$-value data whereas $D^{*}$ is determined primarily by the behaviour at low $b$-values Unfortunately, the Siemens sequence that was used had a fixed increment of 50 $\mathrm{s} / \mathrm{mm}^{2}$ for $b$ As a result, we were limited to only 3 or 4 points in the range of $b$ values that was most important for a good determination of $D^{*}$ This is discussed in more detall in the previous chapter in conjunction with similar experiments performed on the liver

For most of the subjects the amount of variation is about the same for the WP-ROI as it is for the U-ROI, but for patient 5 the variation for the WP-ROI is much larger than it is for the U-ROI Medical assessment with ultrasound for patient 5 determined that the subject was going into preterm labour at the time she had her MR scans and that might have affected the placental processes This could conceivably lead to regional differences in $D^{*}$, explaining this observation However, since this only occurred with one subject, there is not enough experimental evidence to be conclusive The other IVIM parameters did not show a similar behaviour 


\subsubsection{Discussion of IVIM Parameters}

As expected, $D$ does not vary much across the placenta, from subject to subject or with GA since, within the framework of the IVIM model, it is related to the random Brownian motion of the water molecules in the tissue of the placenta and not directly to the circulating blood $D$ is determined primarily by physical characteristics of the tissue such as membrane permeability, cell size and cell density The behaviour of the signal decay for higher $b$-values is dominated by diffusion since the higher amplitude gradients for these $b$-values attenuate the signal from the spins of the placental microcirculation so much that the contribution from these spins is essentially zero

On the other hand, the behaviour of the pseudo-diffusion coefficient, $D^{*}$, and the active capillary fractional volume, $f$, also known as the perfusion fraction, are determined by the combined geometry and dynamics of the fetal capillary network in the placental vill and the maternal blood flow around the vill The perfusion fraction represents the ratio of the volume of MR visible water moving in the capillary compartment (e g water in blood cells, plasma, etc) to the total volume of MR visible water present in the voxel and $D^{*}$ is the rate constant for the diffusion-like displacement of these water molecules

$D^{*}$ is directly related to the speed of the circulating blood and the length of the randomly oriented capillary segments in the villı In a typical voxel there will be many of these randomly oriented capillary segments At the same time, maternal blood in the voxel follows a very tortuous, pseudo-random route as it 
moves around the many branches of the vill 1 The measured value of $D^{*}$ will, therefore, be influenced by the dynamics of both of these blood systems and, in principle, can give very important insight into both the fetal microvasculature of the placental vill and the flow of maternal blood in the intervilous spaces The diffusion-like molecular displacements associated with $D^{*}$ are very complex and it is difficult to predict the value of $D^{*}$ based on known tissue structure and dynamics

After week 12 of the pregnancy, the growth of the placenta parallels fetal growth, so a constant mean perfusion fraction for GA $>12$ wks is to be expected The fractional volume of the intervillous space relative to the total villous plus intervillous volume has been reported to be $(51 \pm 5) \%$ [83] Bergmann et al [84] report that the villous vascular volume is $(20 \pm 6) \%$ of the total villous volume in healthy subjects Thus, the blood in the placenta, including maternal blood in the intervillous spaces and fetal blood in the microvasculature of the villi, should be about $51 \%+20 \% \times 49 \%=61 \%$ of the total placental volume This is somewhat higher that our value of $f=(34 \pm 6) \%$ However, this measured fractional volume should not be expected to be exactly equal to the actual active capillary fractional volume, although they are related, they are not quite the same The measured value of $f$ is the fraction of the diffusion decay that corresponds to the faster decaying component within the IVIM bi-exponential decay model Implicit to this model are the assumptions that 1) there are two independent groups of spins whose diffusion can be characterized by distınct diffusion coefficients, $D$ and $D^{*}$, 2) all of the spins in each compartment contribute to the signal for their group's 
component of the decay curve and 3) the motion of the spins in each group is diffusive in nature and can be modelled as a random-walk process These will be discussed individually in the following paragraphs

The b1-exponential IVIM model assumes that there are two independent groups of spins and that the diffusion of all of the spins in a particular group can be characterized by the same diffusion coefficient which is different from the diffusion coefficient for the other group This is clearly too simplistic for complex biological tissues, however, as long as the behaviour of the spins in the group can be reasonably represented by the mean behaviour for the group and the distribution of diffusive behaviour for the group is Gaussian-like and not too broad, it can be a useful approach This appears to be a reasonable approximation for the diffusing spins in biological tissues, in general, and for the placenta, in particular, however, for the flowing blood group in the placenta it may be less reasonable Certainly in areas near where the blood enters the placenta from the spiral arteries this model may break down but it may be questionable for other areas as well A gradient of mobility exists across the placenta as the distance from the blood entry point increases This distribution of mobilities can be expected to be farly broad and may include regions where the diffusive displacements of the spins in the blood are small and are better characterised by $D$ rather than $D^{*}$ In other words, some of the maternal blood may contribute to each of the diffusion components instead of contributing to only the $D^{*}$ component as is implicitly assumed in the model 
The experimental evidence shows conclusively that the diffusion signal from the placenta can be characterized effectıvely as a b1-exponential process It is very likely that the moving blood, in the microvasculature of the vill and the intervillous spaces, is the primary contribution to the faster decaying component and that diffusing water in the tissue contributes to the slower component only but it is possible that the moving blood may contribute to both components Therefore, makıng quantıtatıve comparısons between IVIM parameters and known anatomical and physiological values must be done with caution

The second assumption of the IVIM model mentioned above is that all of the water spins in the tissue contribute equally to the measured signal decay This may not be accurate when relaxation effects are considered If the $T_{l}$ and $T_{2}$ relaxation tımes are the same for each component then these terms can be factored out of the signal equation to leave the assumed bi-exponential equation for the diffusion decay However, when this is not the case, relaxation factors must be included for each term Since these extra factors are not a function of $b$, they act as we1ghtıng factors for $f$ and $1-f$ If, for example, the $T_{2}^{*}$ value for the component that diffuses more quickly is much smaller than the $T_{2}^{*}$ value for the other component, then the measured value of $f$ will be smaller than would otherwise be expected

Furthermore, there may be a subgroup of spins that relax so quickly that they do not contribute signal at all An example of this would be spins that are in close proximity (possibly hydrogen bonded) to a macromolecular surface that can induce relaxation transitions or paramagnetic centers that can locally alter the 
magnetic field experienced by the spins If such a subgroup is distributed equally between the two components then it should not influence $f$ but otherwise it could have an effect For example, water molecules in the vicinity of deoxyhemoglobin molecules may be strongly affected by the paramagnetic heme group and may not contribute to the measured MR signal because of increased $T_{2}$ and/or $T_{2}^{*}$ relaxation If this effect is strongest among the spins contributing to the $D^{*}$ component then the measured value for $f$ will be smaller than expected based purely on anatomical considerations Note that $T_{l}$ relaxation transitions will also be induced in this example for spins close to the paramagnetic centre but it is unlıkely to have a strong effect on the signal when a single shot EPI sequence is used

The maternal blood as it enters the intervillous space does not immediately undergo pseudo-random motion, as is assumed with the IVIM model It is un1directional until it has interacted with the villi enough to randomise the motion of the water molecules in the blood Spins that move across a voxel in unison will accumulate a phase shift relative to their initial phase but this will have no effect on the measured magnitude of the signal since all of these spins will have the same phase shift In order to induce decay of the signal magnitude, the spins within a voxel must de-phase relative to one another In reality there will be a small amount of de-phasing since not all of the spins will have exactly the same speed and/or direction, however, the effect of such isotropic motion on the signal decay will be much smaller than if this motion within the voxel was completely anısotropic This may be another mechanısm whereby water from moving blood 
may actually contribute to the more slowly decayıng component even though the blood flow is relatively high

While caution must be used when interpretıng the IVIM parameters in terms of absolute anatomical and/or physiological properties, it should be emphasized that a comparison of the IVIM parameters for healthy and unhealthy subject groups is still a very important and useful thing to do While it may be a challenge to measure accurate absolute values for these parameters it is expected that differences in the measured values of these parameters for healthy and unhealthy subjects will be important diagnostic indicators of disease However, an important prerequisite for such an application is a reliable knowledge of the IVIM parameter values for healthy subjects

\subsection{Conclusions}

IVIM parameter values are presented for the placenta that are considerably more reliable than those reported previously This improvement is partly a result of using superior hardware and a significantly better measurement protocol that included parallel ımagıng, navıgator echo respıratory trıggerıng and SPAIR fat suppression A more systematic approach was also used for the analysis relative to the earlier studies The values given here are, for the most part, consistent with the values reported in the literature but they have greatly reduced coefficients of variation (1 e improved precision) compared with the previous studies Where the

values differ, we believe our values to be the more reliable ones None of the IVIM parameters was a function of gestational age 
The IVIM parameters discussed in this chapter were studied in five different ways 1) with three square 100-pixel ROIs carefully placed in the placenta to avold areas where the blood enters the placenta, 2) with an ROI constructed from the union of the three 100-pixel ROIs ( 1 e the U-ROI), 3) with an ROI drawn around the whole placenta ( $1 \mathrm{e}$ the WP-ROI), 4) with ROIs drawn around the inside ( $1 \mathrm{e}$ the fetal side) and the outside ( $1 \mathrm{e}$ the maternal side) of the placenta and 5) with a pixel-by-pixel analysis of the whole placenta from which parametric maps were generated No significant differences were observed for the IVIM parameter values obtained with the 100-pixel ROIs, the U-ROI or the WP-ROI, although there was a trend towards higher values of $f$ and $D^{*}$ for the WP-ROI (see also Table 43 and the tables given in Appendix A) However, the analysis using $\mathrm{ROI}_{\mathrm{out}}$ and $\mathrm{ROI}_{\mathrm{in}}$ showed that both $f$ and $D^{*}$ are larger on the maternal side of the placenta This trend was also seen in the parametric maps for $f$ and $D^{*}$ maps All of these analyses showed that $D$ is the same for all locations within the placenta, to within experimental error IVIM parametric maps can also be useful for identifying variations in tissue structure and dynamics across the placenta Important insights into the hemodynamic systems of the placenta can be gained from these maps The measurement techniques presented here could become instrumental in the assessment and treatment of abnormal pregnancy

All of the patients studied were considered by the obstetricians to have a healthy placenta Thus, the parameter values presented here should characterize placental blood dynamics for normal, healthy placentas However, three of the patients had other complications with their pregnancies All three of these 
subjects had negative $f_{\text {out }}-f_{m}$ values and one of them also had a negative value for $D_{\text {out }}^{*}-D_{\text {in }}^{*}$ (the only negative value found for this parameter) None of the other patients had a negative value for either of these parameters Thus, for our small group of patients a negative $f_{\text {out }}-f_{l n}$ and/or a negative $D_{\text {out }}^{*}-D_{\imath n}^{*}$ value was associated with a patient with a complication with their pregnancy in every case This is consistent with the results presented by the Nottingham group on $f_{\text {out }}-f_{\text {in }}$ for patients with fetal growth restriction $D_{\text {out }}^{*}-D_{m}^{*}$ values have not been reported previously for either healthy or unhealthy human subjects

When comparing the IVIM values with tissue structural information the comparison must be done cautiously Based on information avalable in the literature it was argued that the blood in the placenta should occupy about $61 \%$ of the total placental volume This appears to be in contradiction with our $f$-value of $(34 \pm 6) \%$ However, various reasons are given for why the measured $f$-value, the normalized $y$-intercept on the semi-logarithmic signal decay plots, can be expected to differ from the fractional blood volume of the placenta This parameter is likely related to the blood volume but it is not a direct measure of it

In this chapter significant improvements to the experimental and analysis procedures are presented for studying the IVIM parameters for the placenta As a consequence, the values of the IVIM parameters given here are considerably more reliable than the values previously reported in the literature With these improvements, this approach has significant potential as an important procedure for the diagnosis of placental abnormalities 


\section{Chapter 5}

\section{Rectified Noise Bias Correction}




\subsection{Introduction}

The extraction of the true MR signal intensity ( $1 \mathrm{e}$ the signal intensity that would be obtained in the absence of noise) from noisy MR magnitude images is confounded by a bias caused by noise rectification in the magnitude calculation for pixels with a low signal-to-noise ratio (SNR). This will be referred to in this chapter as rectified noise bias (RNB), in general, and the rectified noise floor (RNF), or simply the noise floor, for the special case where the MR signal is zero The aim of this chapter is to present a useful technique for removing this bias from the pixel intensities of magnitude MR images The method presented here is an extension of the analytical solution given by Koay and Basser [86] and the RNB correction (RNBC) algortthm presented previously for a 1-element coil [87] to an $N$-element phased array coll

The majority of $\mathrm{rf}$ receiver colls on modern clinical MR scanners are phased array colls The use of phased arrays increases the SNR of the measurement without introducing artifacts and is applicable to all pulse sequences without affecting image contrast Scanners with this technology can also use the information from the separate coil elements in the phased array to either acquire the image more quickly or acquire more information (e $\mathrm{g}$ higher resolution images) in the same time This is known to as Parallel Imaging (PI) For some applications in MR, PI very important, however, the use of this method is not always advantageous since the SNR of the resultıng image is reduced

Quantitative Magnetıc Resonance (QMR) [88] is gaining in popularity because it provides an important additional tool for diagnosis, monitoring of 
disease progression and response to treatment With the conventional approach to MRI, certain tissue properties are used to weight the contrast in the images to help distinguish diseased tissue from healthy tissue With $Q M R$, the values of MR parameters that characterize these properties are quantified and clinical decisions are made based on these parameter values The studies presented in chapters 3 and 4 of this thesis are examples of QMR One thing that people must be aware of with many QMR studies is that RNB can distort diffusion decays, relaxation decays and other quantitative measurements when the signal strength is low For diffusion and relaxation decays, the signal attenuation is normally followed from a relatively high SNR value to lower intensity values For complicated mult1component systems it is necessary to measure the attenuation with numerous points along the decay to be able to properly characterize the curve RNB can sometımes introduce errors into the fit parameters computed from the decay [89] or it can be misinterpreted as an extra component on the decay If not dealt with properly, RNB can lead to inaccurate results whether signal detection is performed with a 1-element coil or an $\mathrm{N}$-element phased array coil Note that, although signal averaging can be used to increase the effective SNR, it does not remove the RNB

For studies such as the IVIM assessment of placenta and liver presented in this thesis, RNB imposes a constraint on the study design since, for example, it restricts the $b$-value range and the voxel size ( $1 \mathrm{e}$ the resolution) that can be used

In this chapter, a new Rectıfied Noise Bias Correction (RNBC) method for phased array coils is presented Section 52 is dedicated to a general introduction 
of the relevant theory The extension of the existing algorithm to phased array colls is presented in Section 522 The usefulness of this RNBC technique is demonstrated with simulations The methods used to perform these simulations are described in Section 53, the results are presented in Section 54 and the significance of the algorithm is discussed in Section 55

\subsection{Theory of Rectified Noise Bias Correction}

\subsubsection{Background Theory}

For an $N$-element phased array receiver coll, the noise on the real and imaginary channels for each element in k-space will be statistıcally independent and well described by a Gaussian Probability Density Function (PDF) with a mean of zero as long as the coil is properly designed and well bult The PDFs for the noise of the Fourier transformed real and imaginary signals are also zero mean Gaussians and these PDFs have a standard deviation of $\sigma_{g}$ [90] There have been a few reports in the literature of noise correlations [91,92] but this effect is not common and will not considered in the present study

When the signal detection is performed with an $N$-element phased array, the magnitude image pixel intensities, $M_{N}$, are often computed using a sum of squares algorithm which gives [86]

$$
M_{N}=\sqrt{\sum_{l=1}^{N}\left(M_{l R}^{2}+M_{l I}^{2}\right)} \text { with } \begin{aligned}
& M_{l R}=A_{l R}+n_{l R} \\
& M_{l I}=A_{l I}+n_{l I}
\end{aligned}
$$

where $A_{l R}$ and $A_{l I}$ are the real and imaginary components, respectively, of the true (1 e noiseless) MR induction signal $A$, for the $l^{\text {th }}$ coll element and $n_{I R}$ and $n_{I I}$ are 
the statistically independent noise contributions to the real and imaginary channels for the $l^{\text {th }}$ coll element, respectively From Eq $(51)$ it can be seen that the image pixel intensity in the absence of noise, $A_{N}$, is given by

$$
A_{N}=\sqrt{\sum_{l=1}^{N}\left(A_{l R}^{2}+A_{l I}^{2}\right)}
$$

The calculation of the pixel magnitudes using a sum-of-squares equation (1 e Eq (5 1)) rectifies the noise and transforms the distribution of the data from several sets of Gaussian distributed data to a single non-central chı distribution (NCD) of pixel intensities given by [86]

$$
\mathrm{P}_{\mathrm{M}}\left(M_{N} \mid A_{N}, \sigma_{g}\right)=\frac{A_{N}}{\sigma_{g}^{2}}\left(\frac{M_{N}}{A_{N}}\right)^{N} \exp \left[-\frac{M_{N}^{2}+A_{N}^{2}}{2 \sigma_{g}^{2}}\right] \mathrm{I}_{N-1}\left(\frac{M_{N} A_{N}}{\sigma_{g}^{2}}\right),
$$

where $\mathrm{I}_{N-1}$ is the $(N-1)^{\text {th }}$ order modified Bessel function and the vertical bar in the argument of $\mathrm{P}_{\mathrm{M}}$ denotes a conditional probability The mean of this PDF is shifted away from $A_{N}$ by an amount equal to the RNB This PDF can be thought of as the normalized distribution of pixel intensities for an infinitely large image acquired using a phased array coll where the true, noiseless signal is the same for each pixel and the width of the distribution is due to the presence of noise on the MR signal Equivalently, it can be viewed as the normalized distribution of pixel intensities that would be obtained if the signal from the same voxel was acquired an infinite number of tımes using a phased array coll

The first moment of the NCD, $\mu_{\chi}$, is given by [86]

$$
\mu_{\chi}=\beta_{N 1} \mathrm{~F}_{1}\left(-\frac{1}{2}, N, \frac{-\theta_{N}^{2}}{2}\right) \sigma_{g},
$$


where ${ }_{1} \mathrm{~F}_{1}$ is the confluent hypergeometric function and $\theta_{N} \equiv A_{N} / \sigma_{g} \quad \beta_{N}$ is given by the equation

$$
\beta_{N} \equiv \sqrt{\frac{\pi}{2}} \frac{(2 N-1) !}{2^{N-1}(N-1)^{\prime}}
$$

where the double factorial is defined by $n^{\prime \prime}=n(n-2)(n-4) \quad$ The second moment and the variance of the NCD are given by [86]

$$
\mathrm{E}_{\chi}\left[M_{N}^{2}\right]=A_{N}^{2}+2 N \sigma_{g}^{2}
$$

and

$$
\sigma_{\chi}^{2} \equiv \mathrm{E}_{\chi}\left[M_{N}^{2}\right]-\mu_{\chi}^{2}=\xi\left(\theta_{N}, N\right) \sigma_{g}^{2}
$$

respectively, where $E_{\chi}[]$ signifies the expectation operator calculated over the NCD and the "correction factor", $\xi\left(\theta_{N}, N\right) \equiv \sigma_{\chi}^{2} / \sigma_{g}^{2}$, has been introduced [86] By substituting Eqs (5 4) and (5 6) into Eq (5 7) and solving for $\xi\left(\theta_{N}, N\right)$, it can easily be shown that

$$
\xi\left(\theta_{N}, N\right)=2 N+\theta_{N}^{2}-\left[\beta_{N 1} \mathrm{~F}_{1}\left(-\frac{1}{2}, N,-\frac{\theta_{N}^{2}}{2}\right)\right]^{2}
$$

For the special case where $A_{N}=0$ this reduces to

$$
\xi(0, N)=2 N-\beta_{N}^{2},
$$

since ${ }_{1} \mathrm{~F}_{1}[a, 0, b]=1$ for all values of $a$ and $b[86]$

An expression for $A_{N}$ can be derived by inserting Eq (5 6) into Eq (5 7) and rearranging to get

$$
A_{N}^{2}=\mu_{\chi}^{2}-\left[2 N-\xi\left(\theta_{N}, N\right)\right] \sigma_{g}^{2}=\mu_{\chi}^{2}-q_{N}^{2} \sigma_{g}^{2}
$$


or

$$
A_{N}=\sqrt{\mu_{\chi}^{2}-q_{N}^{2} \sigma_{g}^{2}}
$$

where

$$
q_{N}^{2} \equiv 2 N-\xi\left(\theta_{N}, N\right)
$$

Koay and Basser [86] have shown that when the correct values of $\mu_{\chi}$ and $\sigma_{g}$ are known, exact values of $\xi\left(\theta_{N}, N\right)$ and $A_{N}$ can be evaluated using Eq (5 10) for any $\theta_{N}$ In practice, reliable values for $\sigma_{g}$ can often be calculated from the image background but accurate values for $\mu_{\chi}$ are normally unavallable Thus, in order for $\mathrm{Eq}(510)$ to be useful for the removal of RNB in real situations, approximations must be used for $\mu_{\chi}$ For example, for images with areas of uniform pixel intensity ( 1 e constant $A_{N}$ values), $\mu_{\chi}$ can be approximated by the mean of these pixel intensities The reliability of this approximation will depend on the number of pixels in the region of interest (ROI) used to compute this mean

\subsubsection{Extension of the Rectified Noise Bias Correction Algorithm}

By applying a binomial expansion to $\mathrm{Eq}$ (59), the equation for $A_{N}$ can be transformed from a complex expression involving a square root to a simple linear correction with the correction term $\Delta \mu_{\chi}$ separated from the term to be corrected [87]

$$
A_{N}=\mu_{\chi}-\mu_{\chi}\left[\frac{1}{2}\left(\frac{q_{N} \sigma_{g}}{\mu_{\chi}}\right)^{2}+\frac{1}{8}\left(\frac{q_{N} \sigma_{g}}{\mu_{\chi}}\right)^{4}+\right]=\mu_{\chi}-\Delta \mu_{\chi} \text { for } \frac{\mu_{\chi}}{q_{N} \sigma_{g}}>1
$$


The correction term in Eq (5 12) is exactly equal to the RNB Note that the valıdity condition for the binomial expansion is always satısfied since, using Eqs (5 10) and (5 11) with $A_{N}=0$, it can be shown that $\mu_{\chi}\left(A_{N}\right) \geq \mu_{\chi}(0)=q_{N}(0) \sigma_{g} \geq q_{N}\left(A_{N}\right) \sigma_{g}$ where $\mu_{\chi}$ and $\xi\left(\theta_{N}, N\right)$ are both known to be monotonically increasing functions of $A_{N}[86]$ and $0429 \leq \xi\left(\theta_{N}, N\right) \leq 10$ [86] An expression similar to $\mathrm{Eq}(512)$ has been presented previously for the special case where $N=1$ but it has not yet been studied for $N>1$

Equations (5 10) and (5 12) are mathematically equivalent and they give exactly the same result when an accurate $\mu_{\chi}$ value is used to calculate $A_{N}$ However, when approximate $\mu_{\chi}$ values are used, as is always required in practice, it is sometımes advantageous to use the binomial expansion form of the equation The significance of this will be discussed further in Section 54

When $\mu_{\chi}$ is replaced in Eq (5 12) with an average over $n$ points from a NCD corresponding to the same value of $A_{N}$, which we will write as $\left\langle M_{N}\right\rangle_{\chi n}$, Eq

(5 12) can be re-written as

$$
\begin{array}{r}
\tilde{A}_{N, n^{\prime}, n}=\left\langle M_{N}\right\rangle_{\chi^{\prime}}-\left\langle M_{N}\right\rangle_{\chi^{n}}\left[\frac{1}{2}\left(\frac{q_{N} \sigma_{g}}{\left\langle M_{N}\right\rangle_{\chi^{m}}}\right)^{2}+\frac{1}{8}\left(\frac{q_{N} \sigma_{g}}{\left\langle M_{N}\right\rangle_{\chi^{n}}}\right)^{4}+. .\right] \\
\text { for } \frac{\left\langle M_{N}\right\rangle_{\chi n}}{q_{N} \sigma_{g}}>1
\end{array}
$$

or

$$
\widetilde{A}_{N, n^{\prime}, n}=\left\langle M_{N}\right\rangle_{\not n^{\prime}}-\Delta M_{N, n} \quad \text { for } \frac{\left\langle M_{N}\right\rangle_{\not n}}{q_{N} \sigma_{g}}>1
$$


where the tilde over the $A_{N}$ is used to signify that this is an approximate value of $A_{N}$ and the subscripts indicate that the averaging is performed over $n^{\prime}$ points for the first term and $n$ points for the correction term where $n$ and $n^{\prime}$ are not necessarily equal Note that the binomial validity condition for Eq (5 13) will not always be satisfied since an approxımation to the true PDF mean is used

Equation (5 13) can also be expressed as a recurrence relation

$$
\tilde{A}_{N n^{\prime} n}=\left\langle M_{N}\right\rangle_{\chi n^{\prime}}+\left\langle M_{N}\right\rangle_{\chi n} \sum_{k=1}^{\infty} a_{k-1} \frac{k-\frac{3}{2}}{k}\left(\frac{q_{N} \sigma_{g}}{\left\langle M_{N}\right\rangle_{\chi n}}\right)^{2} \text { for } \frac{\left\langle M_{N}\right\rangle_{\chi^{n}}}{q_{N} \sigma_{g}}>1,
$$

where $a_{0}=1$ and $a_{k-1}$ is the previous term in the series All of the terms in the series are negative which means that the correction term, $\Delta M_{N, n}$, is always positive This expression is much more convenient for computer simulations

\subsection{Methods}

The results reported in this chapter were generated using simulation software written in IDL (ITT Visual Information Solutions, Boulder, CO) The magnitude of each simulated MR signal was calculated using Eq (51) where the $n_{I R}$ and $n_{I I}$ were generated as Gaussian distributed random numbers chosen independently of each other using IDL's RANDOMN procedure For convenience, $\sigma_{g}$ was set equal to one Note that since the results presented in this chapter are a function of the SNR and not the signal or the noise directly, it does not matter what value is used for $\sigma_{g}$ in the simulations $A_{l R}$ and $A_{l l}$ were computed from

$$
A_{l R}=A \cos \phi
$$


and

$$
A_{l l}=A \sin \phi
$$

respectively, where $\phi$ is the phase of the MR receiver, which was assumed to be the same for all coil elements and was arbitrarily set to $\phi=0$ For simplicity, the same value of $A$ was used for each coll element Note there is no loss of generality with these assumptions since the simulation results obtained for any $A_{N}$ value calculated in this way will be equally valid for the same $A_{N}$ value calculated using different values of $A$ for the different coil elements

The NCDs used for these simulations were generated, for given values of $N, A$ and $\sigma_{g}$, as histograms from $N_{s}=1 \times 10^{6}$ simulated noisy MR signal magnitudes using the IDL histogram procedure with 400 bins spanning a range of $\theta_{N}$ values from -10 to 10 The histograms were normalized such that the area under the curve is 10 so that they correspond to PDFs In all cases, the mean of these simulated PDFs was in good agreement with the true mean calculated from Eq (5 4) The RNBC algorithm, for the binomial expansion form of the correction, as given by $\mathrm{Eq}$ (5 14), was initially validated by using the correct values for $\mu_{\chi}$ which were calculated using Eq (5 4)

To test the RNBC algorithm for the more realistic situation where an approximation of the PDF mean is used, simulations were performed using Eq (5 14) with $n=9$ and $n^{\prime}=1$ for $N=1,2,4,8$ and 16 This corresponds to a situation where the RNBC is applied to individual image pixel intensities with the RNB correction term calculated using $\left\langle M_{N}\right\rangle_{x^{9}}$, which can be thought of as the average of the intensities of the pixel being considered and the 8 surrounding 
pixels It is assumed that these nine points all correspond to the same value of $A_{N}$ For the purposes of the simulation, this "9-point nearest neighbour average" was computed as the mean over 9 consecutive $M_{N}$ values for the same value of $A_{N}$

Once a value for $\left\langle M_{N}\right\rangle_{x n}$ has been determıned, the RNBC algorithm uses the iterative procedure presented by Koay and Basser [86], which is valid for $\left\langle M_{N}\right\rangle_{\chi^{n}} \geq \sqrt{2 N-\xi(0, N)} \sigma_{g}=q_{N}(0) \sigma_{g}=\beta_{N} \sigma_{g}$, to evaluate $\xi\left(\theta_{N}, N\right)$, whenever the validity condition is satisfied This computation is terminated when ether the absolute value of the difference between two consecutive iterations is less than or equal to $1 \times 10^{-8}$ or the number of iterations exceeds 100 When this validity condition is violated the algorithm arbitrarily sets

$$
q_{N}^{2}\left(\theta_{N}\right) \rightarrow q_{N}^{2}(0)=2 N-\xi(0, N)=\beta_{N}^{2}
$$

The next step in the procedure is to check the validity condition for the binomial expansion in Eq (5 14) If this condition is satısfied, the correction term is computed and $\widetilde{A}_{N n^{\prime} n}$ is evaluated The series expansion is considered to have converged when either the magnitude of the next term in the expansion is less than $1 \times 10^{-9}$ or the number of terms considered exceeds $25 \times 10^{5}$ It is necessary to consider a large number of terms in the series expansions since these series converge extremely slowly when $\mu_{\chi} \approx q_{N} \sigma_{g}$ or $\left\langle M_{N}\right\rangle_{\chi n} \approx q_{N} \sigma_{g}$

For a given NCD it is to be expected that the binomial expansion condition will be satısfied for some points but violated for others, particularly when $N, \theta_{N}$ and $n$ are small When this condition is violated, neither of the approaches introduced above is strictly valid since they etther involve the square root of a 
negatıve number or, equivalently, a diverging senes When the binomial expansion condition is violated in the linear RNBC method proposed here, the algorithm sets $\Delta M_{N n}=\beta_{N} \sigma_{g}\left(1 \mathrm{e}\right.$ the exact correction for $\mu_{\chi}$ when $\left.A=0\right)$ and calculates $\widetilde{A}_{N{ }_{n}}$ directly from Eq $(513 \mathrm{~b})$ Note that this is not the same as settıng $\tilde{A}_{N n^{\prime} n}=0$ since the approxımation $\Delta M_{N, n}=\beta_{N} \sigma_{g}$ is only used for determining the correction term, $\tilde{A}_{N n^{\prime} n}$ is the difference between $\left\langle M_{N}\right\rangle_{m^{\prime}}$ and $\Delta M_{N n}$ and there are many possible values for $\left\langle M_{N}\right\rangle_{\chi n^{\prime}}$

The simulations are repeated for $N_{s}=1 \times 10^{6}$ simulated pixel intensities The values given in the tables and figures of this chapter are the average over these $N_{s}$ trials, which closely approximates the average calculated over the NCD

\subsection{Results}

To test the effectiveness of the RNBC algorithm, sımulations were performed with noisy MR magnitude signals generated from real and imaginary vectors using Eq (5 1) For all of these simulations, a sum-of-squares equatıon was used to compute the pixel intensities for an $N$-element array and $\sigma_{g}$ was assumed to be the same for all coil elements These assumptions are not required for the simulations but without them the resulting distributions will not strictly follow the NCD given in Eq (5 1) and, since the RNB correction algorthm developed in Section 522 is only theoretically valid for this distribution, it would be more difficult to evaluate the performance of the RNBC algorithm without these 
assumptions In addition, it was assumed that $A$ was the same for all coil elements In this case, it can be seen from Eq (52) that the expression for $A_{N}$ simplifies to $A_{N}=\sqrt{N} A$

Typical uncorrected, simulated distributions are shown in Fig 51 for several values of $A$ for $N=4$ and in Fig 52 for $\theta=05$ for $N=1,2$ and 4 (see the triangular data points) For small $\theta$ values, the PDF deviates noticeably from a Gaussian distribution and the RNB cannot be ignored in cases such as these The distributions shown in these figures are in good agreement with NCD curves published in the literature [91]

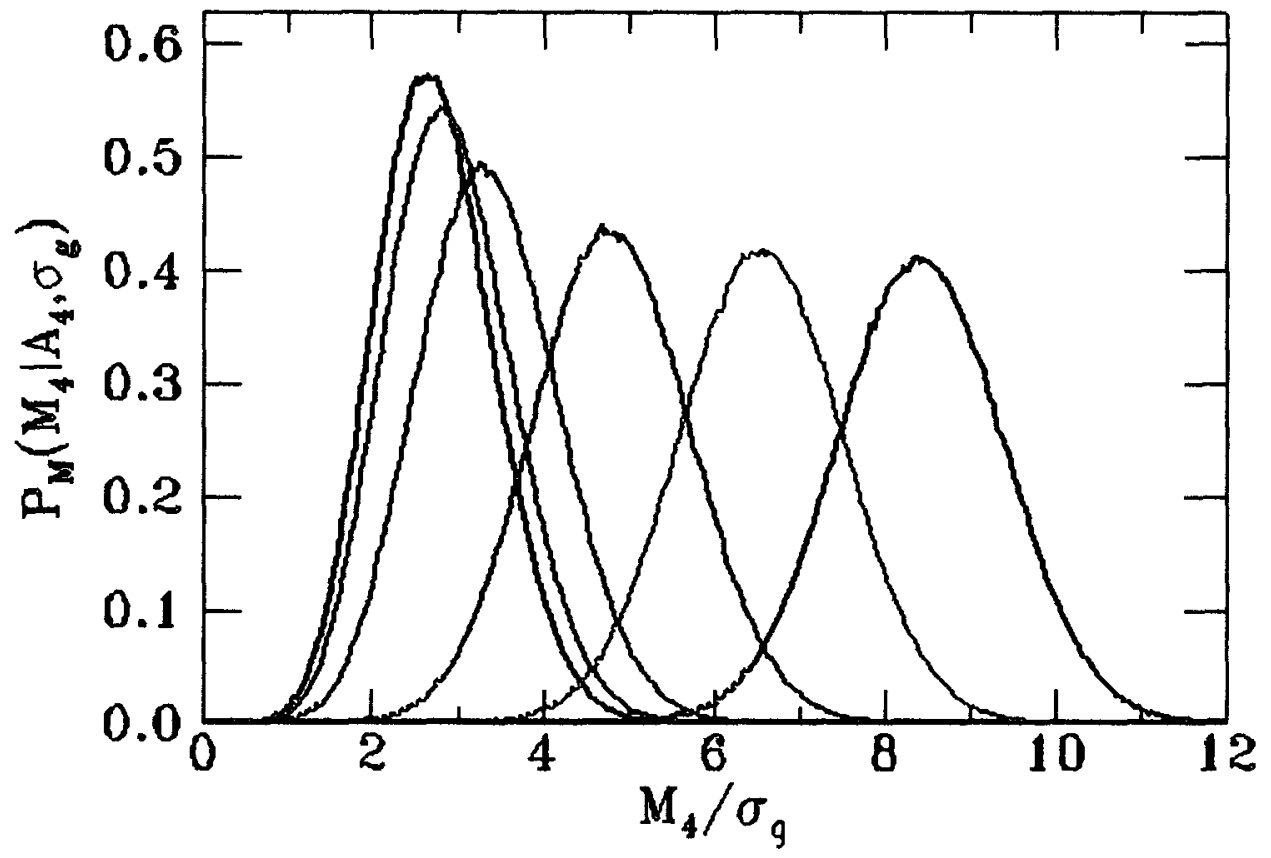

Fig. 5.1 Uncorrected simulated NCDs for several values of $\theta_{N}=A_{N} / \sigma_{g}$ for a 4element phased array and $\sigma_{g}=1$ The black, red, blue, green, purple and dark blue curves correspond to $A=00,05,10,20,30,40$, respectively 
Inttally, the RNBC algorthm was tested using Eq (5 12) with the true NCD mean calculated from Eq (5 4) The results of these tests are summarized in Table 51 and typical RNB corrected NCDs are shown in Fig 52 For each SNR considered, the computed estımate, $\widetilde{\theta}_{N}$, given in Table 51 , is very close to the true $\theta_{N}$ value More complete tables are given in Appendix A for this chapter Note that computed estimates of a parameter are indicated by placing a tilde over the parameter For example, the true SNR is written as $\theta_{v} \equiv A_{N} / \sigma_{g}$ while the corresponding computed estımate is denoted by $\widetilde{\theta}_{N}$

It can also be seen from Fig 52 that, although the RNBC algorithm "corrects" the mean value of the distribution, the shape and variance of each distribution both remain unchanged Analogous behaviour is seen for all values of $A$ and $N$ investigated

Table 5.1 Computed estimates of $\theta_{N}$ as a function $A$ and $N$ for the case where the exact values of $\mu_{x}$ and $\sigma_{g}$ are used

\begin{tabular}{|ccccccccccc|}
\hline$A$ & $\theta_{1}$ & $\widetilde{\theta}_{1}$ & $\theta_{2}$ & $\widetilde{\theta}_{2}$ & $\theta_{4}$ & $\widetilde{\theta}_{4}$ & $\theta_{8}$ & $\widetilde{\theta}_{8}$ & $\theta_{16}$ & $\widetilde{\theta}_{16}$ \\
\hline 0000 & 0000 & 00001 & 0000 & -0046 & 0000 & -0058 & 0000 & -00528 & 0000 & -0042 \\
0500 & 0500 & 0500 & 0707 & 0672 & 1000 & 0954 & 1414 & 1371 & 2000 & 1965 \\
1000 & 1000 & 1001 & 1414 & 1397 & 2000 & 1974 & 2828 & 2802 & 4000 & 3978 \\
2000 & 2000 & 2000 & 2282 & 2828 & 4000 & 3994 & 5657 & 5649 & 8000 & 7991 \\
2500 & 2500 & 2500 & 3536 & 3537 & 5000 & 4996 & 7071 & 7066 & 10000 & 9995 \\
3000 & 3000 & 3000 & 4243 & 4244 & 6000 & 5998 & 8485 & 8483 & 12000 & 11998 \\
4000 & 4000 & 4000 & 5657 & 5678 & 8000 & 7999 & 11314 & 11375 & 16000 & \\
\hline
\end{tabular}




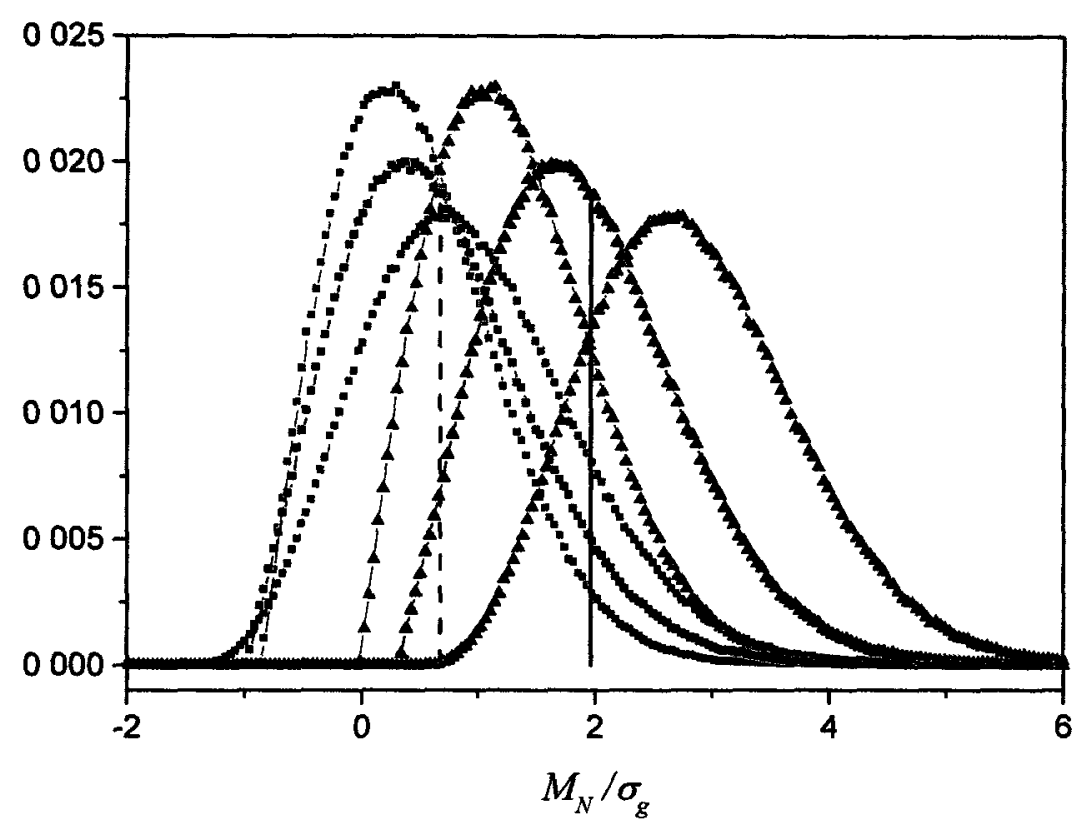

Fig. 5.2. Simulated PDFs before ( $\Delta)$ and after $(\boldsymbol{\square})$ the RNB correction computed using exact values for $\mu_{\chi}$ and $\sigma_{g}=1$, for $N=1$ (red), $N=2$ (black) and $N=4$ (olive) elements and $\theta=05$ The vertical lines show the corresponding PDF means for the corrected (dashed) and uncorrected (solid) PDFs for $N=2$ Note that, for this case, the mean of the corrected PDF is very close to the expected value of 0707

In the next section, the performance of the proposed RNBC algorithm is evaluated as a function of $n$, the number of nearest neighbour pixels averaged together The introduction of nearest neighbour averaging allows for the calculation of the correction term without averaging the term to be corrected This method provides very good RNBC with minimal PDF distortion The results are found in Appendices B and C 


\subsection{Discussion}

Extraction of accurate MR signal intensities from low SNR magnitude MR images has been a challenge for a long time Recently, there has been growing interest in obtaining accurate values for tissue parameters such as diffusion coefficients and relaxation tımes To get reliable information from measured decay curves, a good understanding of the noise and a robust algortthm for dealıng with the RNB are important For decays that are non-exponential or a sum of exponentials, the rectffied noise often perturbs part of the decay and affects the quantitative interpretation of the decay as a result For example, if a decay is assumed to be mono-exponentral and only a few low $b$-values are considered (as is very often done in practice to avold RNB) when the decay is actually bi-exponentral, the decay constant evaluated using only these high SNR points will be incorrect Conversely, if a decay is actually mono-exponential but it is treated as a b1-exponential decay curve because the RNB effects have not been properly identified, this can also lead to an incorrect interpretation of the decay

Early attempts at removing the RNB from magnitude MR umages were only partially successful and have not been widely adopted, with people frequently optıng instead to restrict their measurements to low $b$-values only, to avold data points affected by the RNB Recently, a significant advance was made by Koay and Basser [86] who presented an analytically exact solution for $N$ element phased array colls for the case where both $\mu_{\chi}$ and $\sigma_{g}$ are known 
Unfortunately, for most situations of interest in vivo, reliable $\mu_{\chi}$ values are not avalable and approximations must be used

In this chapter it is shown that the solution given by Koay and Basser for $N$-element phased array colls can be transformed into a very useful RNB correction technique that can be applied to individual magnitude image pixels by using a binomial expansion to linearize their equation This removes the square root operation and separates the correction term from the term to be corrected It also allows the introduction of nearest neighbour averaging to calculate the correction term without averaging the term to be corrected (although this could also be done)

The simulation results presented in this chapter verify that the method works well For the case where $N=1$, the results reported here are in very good agreement with previously reported values For $\theta \geq 10$ and $n=9$ the corrected values are accurate to within $10 \%$ error The new RNB correction algorithm presented here significantly outperforms all previously reported RNB correction techniques for images acquired with phased array coils This RNBC algorithm provides very good RNB correction with minimal PDF distortion, even when $n$ is small

In the ideal case, the linear correction term is the same for all pixel intensities, the original NCD is shifted to lower $\theta_{N}$ values with its shape completely undistorted and the mean of the corrected PDF is exactly equal to $A_{N}$ When a nearest neighbour average is used, a distribution of corrections occurs which leads to small inaccuracies in the estımates of $A_{N}$ and minor distortions of 
the PDF shape The main limitation of the technique is that, when $\left\langle M_{N}\right\rangle_{\not n}<\beta_{v} \sigma_{g}, \xi\left(\theta_{N}, N\right)$ cannot be calculated directly In this case, the algorithm uses the approximation $\xi\left(\theta_{N}, N\right)=\xi(0, N)$ Even so, for $n=9$ the method works very well and PDF distortion is minımal

It should be noted that the RNB correction technique presented here corrects for the rectified noise bias but it does not remove the spread in data points caused by noise The corrected data set will have approximately the same distribution about the corrected PDF mean as the original data set had about the uncorrected PDF mean Consequently, when $A<\sigma_{g}$, the corrected data may contain "negative magnitude" data This should not be of concern since it actually corresponds to the sum of a small positive magnitude signal and a larger negative noise contribution

The RNBC results presented here consider only the correction of individual magnitude image pixels from MR images acquired with $N$-element phased array coils and no signal averaging From Eq (5 13) it is clear that the RNB correction can also be used with averaged signals where the signal to be corrected and the correction term need not be calculated using the same type of averaging (e g signal averaging, nearest neighbour averaging) or the same amount (e g $n^{\prime} \neq n$ ) of averaging 


\subsection{Conclusion}

This chapter presents a method to estımate the RNB from magnitude MRI images when an $\mathrm{N}$-element phased array coll is used The recently published work of Koay and Basser [86] gives an analytically exact correction scheme for this case and is an important advancement However, since their method requires accurate values for both the mean and standard deviation of the non-central Ch1 PDF, it is not practical for in vivo situations By using a binomial expansion of their expression, with the use of a convergence criterion and introducing nearest neighbour averaging, the new correction scheme described here provides very good RNB correction with minımal PDF distortion for all cases when an $N$ element phased array coll is used 
Chapter 6

\section{General Conclusions}


Diffusion-werghted imaging (DWI) is the most important and interesting technıque for non-ınvasively investigatıng water transport in biological tıssues in vivo Using this technique water molecules are tagged and tracked without perturbing their environment or their motion DWI can also be used to track the motion of water in the microvasculature of tissues when the orientation of these tıny blood vessel segments are short and randomly distributed withın the voxel being considered Under these conditions the water motion in the microvasculature, viewed macroscopically, closely approximates a diffusion process A DWI measurement of highly vascularised organs such as the liver and the placenta y1elds a bi-exponential decay where one component corresponds to the water diffusion and the other is associated with slowly flowing blood in the tissue microvasculature (1 e pseudo-diffusion) This approach $1 \mathrm{~s}$ known as the Intravoxel Incoherent Motion model and the three fit parameters obtained from fittıng the measured diffusion decay to a bi-exponential function are called the IVIM parameters

The IVIM model has not often been used in the past for studying abdomınal or pelvic organs, mainly because of the difficulty of obtainıng artefact free DW 1mages, due to the presence of respiratory motion In this thesis, a superior approach to DWI is used to obtain high quality DW images and reliable diffusion decay curves This procedure was used for the IVIM studies of the liver and the placenta reported in Chapters 3 and 4 of this thesis, respectively

By usıng the better ımaging technıque, state-of-the-art scanner hardware and a superior approach to the data analysis we were able to perform more 
accurate and precise IVIM parameter quantification compared with other studies previously reported in the literature for both the placenta and the liver We also showed that a free breathıng protocol using a navigator echo respıratory triggered protocol is far superior to a breath hold protocol for measuring the IVIM parameters in the liver

This is the first study where a whole right liver ROI was investigated and thus we can report, for the first tıme, average IVIM parameter values for the whole right side of the liver This is also the first IVIM study where the entire volume of placenta was investigated and the corresponding IVIM parameter values reported Furthermore, the parametric maps for the IVIM parameters for both the liver and the placenta clearly show changes in $f$ and $D^{*}$ that were consistent with blood flow patterns in these organs

IVIM parameters evaluated with a navigator echo free breathing protocol have significant potential as both sensitive and specific parameters for the detection and diagnosis of hepatıc and/or placental disease However, obtaınıng reliable DW images from which these parameters can accurately be obtained has been a significant problem in the past With the approach presented in this thesis we were able to overcome this problem and report accurate IVIM parameter values for both normal liver and healthy placenta tissue Both of these studies represent significant advancements and are an important first step toward the use of IVIM parameters in MRI studies of the abdomen, particularly for the investigated organs The parametric maps, which showed good anatomical detall, also have considerable promıse as useful visual diagnostıc tools for radıologists to 
help them identify differences in tissues and obtain important insights about the hemodynamic systems present in the tissue This technique could become instrumental in the assessment and management of abdominal pathologies

One limitation of this approach is that low signal to noise ratio (SNR) points on the diffusion decay are perturbed by Rectified Noise Bias (RNB) To avold getting biased data, experiments are designed to ensure that the SNR remains high enough that the RNB has a negligible effect This means that large $b$-values and small voxels cannot be used A method is presented in Chapter 5 of this thesis for overcoming this restriction by correcting the data for the effect of the RNB for image acquisition performed with phased array coils such as were used for the experimental work reported in this thesis Although it was not necessary to use this RNB correction algorithm for the experımental data reported here, the use of RNB correction would have allowed us to extend our measurements to obtain, for example, higher resolution parametric maps

As a final thought, this thesis has focused on the water diffusion present in the tissue microvasculature of highly vascular tissues such as the liver and the placenta where the IVIM model is best suited Diffusion has proven to be a promising source of contrast in MRI and DWI is now an important tool for diagnosis, monitoring of disease progression and response to treatment The extension of DWI to the study of blood flow in the microvasculature of abdominal organs, as reported in this thesis, also shows significant promise 


\section{APPENDIX A: LIVER Statistics}

The tables in this appendix contain descriptive statistics, normality tests for the sample distributions investigated and mean comparison tests Whenever one parameter distribution has not met the normality condition, the non-parametric tests for the appropriate mean comparison have been determined as well $p$-values for both parametric and non-parametric tests are reported

Table A.1 Descriptive Statistics of the ROI Signal Intensity for an arbitrarily chosen slice10 for Patient 5, in the case of a 100 pixel-ROI and the Whole Right Liver (WRL)-ROI for all 13 protocol $b$-values

\begin{tabular}{|c|c|c|c|c|c|c|c|c|}
\hline $\begin{array}{c}b- \\
\text { value } \\
s\end{array}$ & Mean_WR & $S D_{L} W R$ & sk_WR & k_WR & $\begin{array}{l}\text { Mean } \\
100 \mathrm{plx} \\
\text { ROI }\end{array}$ & $\begin{array}{c}\mathrm{SD} \\
100 \mathrm{pl} \\
x \\
\mathrm{ROI}\end{array}$ & $\begin{array}{c}\text { sk } \\
100 \mathrm{pl} \\
x \\
\text { ROI }\end{array}$ & $\begin{array}{c}\mathrm{k} \\
10 \overline{\mathrm{pl}} \\
\mathrm{x} \\
\mathrm{ROI} \\
\end{array}$ \\
\hline 0 & 319 & 149 & 207 & 669 & 517 & 216 & 067 & 319 \\
\hline 1 & 171 & 50 & -001 & 039 & 205 & 33 & 008 & 171 \\
\hline 2 & 156 & 52 & 050 & 093 & 186 & 33 & -008 & 156 \\
\hline 3 & 145 & 45 & 010 & 014 & 191 & 36 & -013 & 145 \\
\hline 4 & 131 & 50 & 001 & 015 & 174 & 31 & 005 & 131 \\
\hline 5 & 131 & 45 & 010 & 009 & 160 & 30 & -007 & 131 \\
\hline 6 & 120 & 40 & 009 & 010 & 144 & 35 & -025 & 120 \\
\hline 7 & 97 & 43 & 013 & -001 & 126 & 22 & -015 & 97 \\
\hline 8 & 105 & 43 & -002 & -037 & 152 & 30 & -025 & 105 \\
\hline 9 & 101 & 42 & 001 & -021 & 124 & 33 & 031 & 101 \\
\hline 10 & 100 & 41 & 020 & -016 & 119 & 26 & 015 & 100 \\
\hline 11 & 86 & 38 & 018 & -021 & 120 & 31 & -032 & 86 \\
\hline 12 & 74 & 34 & 035 & -005 & 76 & 27 & 006 & 74 \\
\hline
\end{tabular}

Table A.2 Descriptive Statistics of the ROI Signal Intensity for 5 consecutive slices for Patient 3 in case of one of the 100 pixel-ROIs and the Whole Right Liver (WRL)-ROI for $b=50 \mathrm{~s} / \mathrm{mm}^{2}$

\begin{tabular}{rrrrrrrrr}
\hline $\begin{array}{c}\text { Slice } \\
\text { number }\end{array}$ & Mean_WRL & SD_WRL & sk_WRL & k_WRL & $\begin{array}{c}\text { Mean } \\
\text { 100pix } \overline{R O I}\end{array}$ & $\begin{array}{c}\text { SD } \\
100 \overline{p I x} \\
\text { ROI }\end{array}$ & $\begin{array}{c}\text { sk } \\
100 \overline{p I x} \\
\text { ROI }\end{array}$ & $\begin{array}{c}k \\
100 \overline{p I x} \\
\text { ROI }\end{array}$ \\
\hline 8 & 175 & 71 & 062 & 144 & 210 & 44 & -013 & 009 \\
9 & 191 & 66 & 054 & 184 & 268 & 53 & 020 & -059 \\
10 & 203 & 72 & 060 & 106 & 278 & 75 & 074 & 090 \\
11 & 189 & 78 & 118 & 349 & 264 & 74 & 052 & 080 \\
12 & 184 & 65 & 025 & 064 & 283 & 44 & -008 & -058 \\
\hline
\end{tabular}

Note Data are reported as mean, SD, skewness (sk) and kurtosıs (k) for each investigated ROI 
Table A.3 Descriptive Statistics of the ROI Signal Intensity for the Central Slice (CS) of each subject(Vol) in the distribution, in case of one of the 100 pixel-ROIs and the Whole Right Liver (WRL)-ROI for $b=50 \mathrm{~s} / \mathrm{mm}^{2}$

\begin{tabular}{|c|c|c|c|c|c|c|c|c|c|}
\hline $\begin{array}{c}\text { Vo } \\
1 \\
\#\end{array}$ & $\begin{array}{l}C \\
S\end{array}$ & Mean_WR & $S D_{L} W R$ & sk_WR & k_WR & $\begin{array}{l}\text { Mean } \\
\text { 100pIX } \\
\text { ROI }\end{array}$ & $\begin{array}{c}\mathrm{SD} \\
100 \mathrm{pl} \\
\mathrm{x} \\
\text { ROI }\end{array}$ & $\begin{array}{c}\text { sk } \\
100 \mathrm{pl} \\
x \\
\mathrm{ROI}\end{array}$ & $\begin{array}{c}\mathrm{k} \\
100 \overline{p I} \\
x \\
\text { ROI }\end{array}$ \\
\hline 1 & 11 & 941 & 458 & 1219 & $\begin{array}{l}6052 \\
1882\end{array}$ & 798 & 286 & 0630 & 1182 \\
\hline 2 & 10 & 3126 & 2886 & 3582 & 6 & 1931 & 628 & 0238 & 0540 \\
\hline 3 & 10 & 2028 & 716 & 0605 & $\begin{array}{l}1062 \\
1497\end{array}$ & 2778 & 747 & 0740 & 0901 \\
\hline 4 & 10 & 2692 & 906 & 2204 & 2 & 3575 & 536 & 0120 & 0175 \\
\hline 5 & 10 & 1712 & 502 & -0014 & 0394 & 2047 & 333 & 0080 & 0437 \\
\hline 6 & 9 & 1919 & 612 & 0203 & $\begin{array}{l}0185 \\
1035\end{array}$ & 1986 & 375 & 0387 & 0683 \\
\hline 7 & 10 & 1348 & 566 & 1623 & $\begin{array}{r}0 \\
4120\end{array}$ & 1461 & 320 & 0048 & 0428 \\
\hline 8 & 10 & 1051 & 667 & 1497 & 4 & 1060 & 347 & 0046 & 0306 \\
\hline
\end{tabular}

Table A.4 Mean $D, D^{*}$, and $f$ values for liver FB-protocol for parametric and non-parametric mean comparison tests A two talled asymptotically significant $p$ value is reported Mean distributions for WP-ROI and U-ROI are compared

\begin{tabular}{ccc}
\hline IVIM Parameter & $\begin{array}{c}t \text {-test } p \text {-value } \\
\text { Parametric }\end{array}$ & $\begin{array}{c}\text { Mann-Whitney } p \text {-value } \\
\text { Non-Parametric }\end{array}$ \\
\hline$D$ & 0250 & 0238 \\
$D^{*}$ & 1 & 0973 \\
$f$ & 0294 & 0439 \\
\hline
\end{tabular}

Note Data are reported as mean \pm SD (if $p$-value $<005$, then results are statıstically different)

Table A.5 A normality test was performed on the population containing the WRL-ROI and PRL-ROI from all the slices for Patient 3 - liver FB-protocol A two talled asymptotic significance $p$-value is reported

\begin{tabular}{|l|r|r|r|}
\hline & \multicolumn{3}{|c|}{ Shapıro-Wilk } \\
\hline & Statıstıc & df & \multicolumn{1}{|c|}{ Sıg } \\
\hline WRL_D & 864 & 10 & 086 \\
WRL_F & 952 & 10 & 692 \\
WRL_Dstar & 806 & 10 & 017 \\
PRL_D & 893 & 10 & 183 \\
PRL_F & 932 & 10 & 470 \\
PRL_Dstar & 944 & 10 & 603 \\
ROI1_D & 893 & 10 & 183 \\
ROI1_F & 932 & 10 & 470 \\
ROI1_Dstar & 944 & 10 & 603 \\
\hline
\end{tabular}

* This is a lower bound of the true significance 
a Lilliefors Significance Correction

Normality assumption can be rejected if $p$-value $<005$ 
Table A.6 Descriptive Statistics for Patient 3, in case of WRL-ROI, PRL-ROI and a 100 pixel-ROI=ROI1 and for $b=50 \mathrm{~s} / \mathrm{mm}^{2}$

\begin{tabular}{|c|c|c|c|c|c|c|c|c|c|c|}
\hline & WRL_D & WRL $F$ & WRL_Dst & PRL D & PRL F & PRL Dst & ROI1_D & ROI1 F & ROI1_Dstar \\
\hline \multirow[t]{2}{*}{$\mathbf{N}$} & Valid & 10 & 10 & 10 & 30 & 30 & 29 & 10 & 10 & 10 \\
\hline & Missing & 20 & 20 & 20 & 0 & 0 & 1 & 20 & 20 & 20 \\
\hline \multicolumn{2}{|l|}{ Mean } & 9915 & 511480 & 453990 & 9420 & 579490 & 397903 & 11611 & 586190 & 430270 \\
\hline \multicolumn{2}{|c|}{ Std Error of Mean } & 04672 & 207583 & 887038 & 05376 & 170187 & 395218 & 10937 & 276596 & 656747 \\
\hline \multicolumn{2}{|l|}{ Median } & 10420 & 506600 & 372000 & 9075 & 565550 & 377400 & 11365 & 561350 & 412150 \\
\hline \multicolumn{2}{|l|}{ Mode } & $76(a)$ & 4220 (a) & $1768(a)$ & 80 & $4391(a)$ & $1256(a)$ & $72(a)$ & $4600(a)$ & 14 53(a) \\
\hline \multicolumn{2}{|c|}{ Std Deviation } & 14775 & 656434 & 2805061 & 29447 & 932155 & 2128314 & 34585 & 874672 & 2076818 \\
\hline \multicolumn{2}{|l|}{ Varıance } & 022 & 43091 & 786837 & 087 & 86891 & 452972 & 120 & 76505 & 431317 \\
\hline \multicolumn{2}{|l|}{ Skewness } & -594 & 730 & 1935 & 697 & 429 & 1005 & -006 & 308 & 555 \\
\hline \multicolumn{2}{|c|}{ Std Error of Skewness } & 687 & 687 & 687 & 427 & 427 & 434 & 687 & 687 & 687 \\
\hline \multicolumn{2}{|l|}{ Kurtosis } & -1452 & 530 & 4383 & 230 & -818 & 1009 & -1880 & -1167 & 453 \\
\hline \multicolumn{2}{|c|}{ Std Error of Kurtosis } & 1334 & 1334 & 1334 & 833 & 833 & 845 & 1334 & 1334 & 1334 \\
\hline \multicolumn{2}{|l|}{ Range } & 38 & 2230 & 9752 & 116 & 3169 & 8715 & 88 & 2580 & 6929 \\
\hline \multicolumn{2}{|l|}{ Mınımum } & 76 & 4220 & 1768 & 44 & 4391 & 1256 & 72 & 4600 & 1453 \\
\hline \multicolumn{2}{|l|}{ Maxımum } & 115 & 6450 & 11520 & 160 & 7560 & 9971 & 160 & 7180 & 8382 \\
\hline \multicolumn{2}{|l|}{ Sum } & 992 & 51148 & 45399 & 2826 & 173847 & 115392 & 1161 & 58619 & 43027 \\
\hline \multirow[t]{3}{*}{ Percentlles } & 25 & 8140 & 458725 & 266700 & 7563 & 508125 & 228450 & 7925 & 513750 & 287875 \\
\hline & 50 & 10420 & 506600 & 372000 & 9075 & 565550 & 377400 & 11365 & 561350 & 412150 \\
\hline & 75 & 11215 & 551450 & 580400 & 10445 & 649575 & 551550 & 15010 & 660800 & 590075 \\
\hline
\end{tabular}


Table A.7 A normality test was performed on the IVIM parameters for WRL-ROI and PRL-ROI from all subjects investigated with the FB-protocol A two tailed asymptotic significance $p$-value is reported

\begin{tabular}{|l|r|r|r|}
\hline & \multicolumn{3}{|c|}{ Shapıro-Wilk } \\
\hline & Statıstic & \multicolumn{1}{|c|}{ df } & \multicolumn{1}{c|}{ Sig } \\
\hline WRL_D & 970 & 11 & 889 \\
PRL_D & 986 & 11 & 991 \\
ROI1_D & 892 & 11 & 149 \\
WRL_Dst & 912 & 11 & 256 \\
PRL_Dst & 676 & 11 & 000 \\
ROI1_Dst & 706 & 11 & 001 \\
WRL_f & 835 & 11 & 027 \\
PRL_f & 878 & 11 & 097 \\
ROI1_f & 846 & 11 & 038 \\
" This Is a lower bound of the true significance \\
a Lilliefors Significance Correction \\
Normality assumption can be rejected if p-value<0 05
\end{tabular}


Table A.8 Descriptive Statistics of the parameter distributions, when all liver subjects are investigated, for WRL-ROI, PRL-ROI and a 100 pixel-ROI $=$ ROI 1 for $b=50 \mathrm{~s} / \mathrm{mm}^{2}$

Descriptive Statıstıcs

\begin{tabular}{|l|r|r|r|r|r|r|r|r|r|}
\hline & \multicolumn{1}{|c|}{$\mathrm{N}$} & \multicolumn{1}{c|}{ Mınımum } & Maxımum & \multicolumn{1}{c|}{ Mean } & \multicolumn{1}{c|}{ Std } & \multicolumn{2}{|c|}{ Skewness } & \multicolumn{2}{c|}{ Kurtosis } \\
\cline { 2 - 9 } & Statıstıc & \multicolumn{1}{c|}{ Statıstıc } & \multicolumn{1}{c|}{ Statıstıc } & Statıstıc & Statıstıc & \multicolumn{1}{c|}{ Statıstıc } & Std Error & Statıstıc & Std Error \\
\hline WRL_D & 11 & 93 & 129 & 11134 & 11224 & 011 & 661 & -591 & 1279 \\
PRL_D & 11 & 89 & 125 & 10580 & 10103 & 177 & 661 & 328 & 1279 \\
ROI1_D & 11 & 96 & 118 & 10820 & 07931 & -287 & 661 & -1719 & 1279 \\
WRL_Dst & 11 & 3600 & 7513 & 523776 & 1251460 & 725 & 661 & -624 & 1279 \\
PRL_Dst & 11 & 3575 & 10332 & 526028 & 1795566 & 2607 & 661 & 7693 & 1279 \\
ROI1_Dst & 11 & 3997 & 10777 & 557300 & 2123509 & 1917 & 661 & 3105 & 1279 \\
WRL_f & 11 & 2339 & 4853 & 413029 & 795204 & -1384 & 661 & 1384 & 1279 \\
PRL_f & 11 & 2194 & 4755 & 385544 & 829255 & -1100 & 661 & 498 & 1279 \\
ROI1_f & 11 & 2541 & 4944 & 394945 & 734350 & -1147 & 661 & 853 & 1279 \\
Valıd N (lıstwise) & 11 & & & & & & & & \\
\hline
\end{tabular}




\section{APPENDIX B: PLACENTA Statistics}

The tables in this appendix contain descriptive statıstics, normality tests for the sample distributions investigated and mean comparison tests Whenever one parameter distribution has not met the normality condition, the non-parametric tests for the appropriate mean comparison have been effectuated as well Both parametric and non parametric p-values are reported

Table B.1 Descriptive Statistics of the ROI Signal Intensity for slice 19, Patient 2 , in case of one of the 100 pixel-ROIs and the Whole Placenta (WP)-ROI for all 13 protocol $b$-values

\begin{tabular}{|c|c|c|c|c|c|c|c|c|}
\hline $\begin{array}{c}b- \\
\text { values }\end{array}$ & Mean_WP & SD_WP & sk_WP & k_WP & $\begin{array}{c}\text { Mean } \\
\text { 100pIx } \overline{R O I}\end{array}$ & $\begin{array}{c}\text { SD_ } \\
\text { 100pix } \\
\text { ROI }\end{array}$ & $\begin{array}{c}\text { sk } \\
100 \overline{p I x} \\
\text { ROI }\end{array}$ & $\begin{array}{c}\mathrm{k} \\
100 \mathrm{p} \mid \mathrm{x} \\
\mathrm{ROI}\end{array}$ \\
\hline 0 & 1133 & 286 & 057 & 329 & 1229 & 130 & -118 & 266 \\
\hline 1 & 600 & 329 & 007 & -096 & 849 & 237 & -103 & -016 \\
\hline 2 & 519 & 285 & 022 & -082 & 816 & 192 & -121 & 082 \\
\hline 3 & 430 & 237 & 004 & -118 & 641 & 155 & -095 & -017 \\
\hline 4 & 352 & 185 & 002 & -117 & 502 & 132 & -092 & -037 \\
\hline 5 & 324 & 169 & 013 & -103 & 525 & 109 & -122 & 097 \\
\hline 6 & 279 & 148 & 026 & -086 & 491 & 101 & -094 & 120 \\
\hline 7 & 236 & 165 & 070 & -050 & 424 & 151 & -071 & -076 \\
\hline 8 & 186 & 120 & 043 & -095 & 323 & 77 & -122 & 087 \\
\hline 9 & 213 & 114 & 027 & -083 & 362 & 84 & -103 & 112 \\
\hline 10 & 182 & 102 & 025 & -088 & 308 & 67 & -086 & 000 \\
\hline 11 & 165 & 100 & 029 & -107 & 288 & 73 & -042 & 095 \\
\hline 12 & 99 & 69 & 090 & 018 & 206 & 61 & -029 & 008 \\
\hline
\end{tabular}

Table B.2 Descriptive Statistics of the ROI Signal Intensity for 5 consecutive slices for Patient 9, in case of one of the 100 pixel-ROIs and the Whole Placenta (WP)-ROI for $b=50 \mathrm{~s} / \mathrm{mm}^{2}$

\begin{tabular}{|c|c|c|c|c|c|c|c|c|}
\hline $\begin{array}{c}\text { Slice } \\
\text { numbe } \\
r\end{array}$ & $\underset{P}{\text { Mean_W }}$ & $S D_{\bar{P}} W$ & sk_W & $k_{-}-W$ & $\begin{array}{l}\text { Mean } \\
\text { 100pIX } \\
\text { ROI }\end{array}$ & $\begin{array}{c}\text { SD_ } \\
100 \mathrm{pl} \\
x \\
\text { ROI }\end{array}$ & $\begin{array}{c}\mathrm{sk} \\
100 \mathrm{pl} \\
x \\
\text { ROI }\end{array}$ & $\begin{array}{c}\mathbf{k} \\
100 \overline{p l} \\
x \\
\text { ROI }\end{array}$ \\
\hline 7 & 699 & 377 & 113 & 261 & 1218 & 172 & 040 & -060 \\
\hline 8 & 585 & 279 & 000 & 019 & 865 & 128 & 021 & 082 \\
\hline 9 & 494 & 204 & -008 & -062 & 723 & 74 & 000 & 022 \\
\hline 10 & 452 & 200 & -020 & -098 & 618 & 98 & -059 & -060 \\
\hline 11 & 422 & 180 & -008 & -030 & 481 & 233 & -039 & -108 \\
\hline
\end{tabular}


Note Data are reported as mean, SD, skewness (sk) and kurtosis (k) for each investıgated ROI

Table B.3 Descriptive Statistics of the ROI Signal Intensity for the Central Slice (CS) of each subject in the distribution, in case of one of the 100 pixel-ROIs and the Whole Placenta (WP)-ROI for $b=50 \mathrm{~s} / \mathrm{mm}^{2}$

\begin{tabular}{|c|c|c|c|c|c|c|c|c|c|}
\hline $\begin{array}{c}\text { Vol } \\
\#\end{array}$ & CS & Mean_WP & SD_WP & sk_WP & k_WP & $\begin{array}{c}\text { Mean } \\
\text { 100pIx ROI }\end{array}$ & $\begin{array}{c}\text { SD } \\
100 p 1 x \\
\text { ROl }\end{array}$ & $\begin{array}{l}\text { sk } \\
100 \bar{p} 1 x \\
\text { ROI }\end{array}$ & $\begin{array}{l}\mathrm{k} \\
100 \overline{p l x} \\
\text { ROl }\end{array}$ \\
\hline 2 & 17 & 658 & 337 & 023 & 018 & 841 & 357 & 086 & 219 \\
\hline 3 & 10 & 748 & 286 & -002 & -046 & 946 & 369 & -077 & -038 \\
\hline 4 & 11 & 693 & 152 & -108 & 157 & 682 & 77 & -048 & 094 \\
\hline 5 & 12 & 680 & 218 & -044 & -016 & 676 & 133 & -081 & -003 \\
\hline 6 & 9 & 696 & 228 & -033 & -014 & 808 & 140 & -005 & -050 \\
\hline 7 & 10 & 660 & 244 & -047 & -020 & 670 & 147 & -081 & -015 \\
\hline 8 & 11 & 502 & 226 & 006 & -030 & 797 & 84 & 044 & 003 \\
\hline 9 & 9 & 744 & 238 & -007 & -030 & 903 & 90 & -033 & -109 \\
\hline
\end{tabular}

Note Data are reported as mean, SD, skewness (sk) and kurtosis (k) for each investıgated ROI

Table B.4 Mean $D, D^{*}$, and $f$ values for placenta FB-protocol for parametric and non-parametric mean comparison tests A two tailed asymptotically significanct $p$-value is reported Mean distributions for WP-ROI and U-ROI are compared

\begin{tabular}{ccc}
\hline IVIM Parameter & $\begin{array}{c}t \text {-test } p \text {-value } \\
\text { Parametric }\end{array}$ & $\begin{array}{c}\text { Mann-Whitney } p \text {-value } \\
\text { Non-Parametric }\end{array}$ \\
\hline$D$ & 0074 & 0095 \\
$D^{*}$ & 0074 & 0090 \\
$f$ & 0056 & 0059 \\
\hline
\end{tabular}

Note Data are reported as mean \pm SD (if $p$-value $<005$, then results are significantly different)

Table B.5 A normality test was performed on the population containıng the WPROI and U-ROI from all the slices for Patient 9 A two talled asymptotic significance $p$-value is reported

\begin{tabular}{|l|r|r|r|}
\hline & \multicolumn{3}{|c|}{ Shapıro-Wik } \\
\hline & Statıstic & \multicolumn{1}{|c|}{ df } & P-value=Sıg \\
\hline WP_D & 986 & 12 & 997 \\
WP_F & 912 & 12 & 229 \\
WP_Dstar & 876 & 12 & 078 \\
UROI_D & 938 & 12 & 470 \\
UROI_F & 928 & 12 & 356 \\
UROI_Dstar & 771 & 12 & 004 \\
ROI1_D & 929 & 12 & 370 \\
ROI1_F & 938 & 12 & 471 \\
ROI1_Dstar & 765 & 12 & 004 \\
\hline
\end{tabular}

* This is a lower bound of the true significance a Lilliefors Significance Correction Normality assumption is rejected if $p$-value $<005$ 
Table B.6 Descriptive Statıstics for Patıent 9, in case of WP-ROI, U-ROI and a 100 pixel-ROI=ROI1 and for $b=50 \mathrm{~s} / \mathrm{mm}^{2}$

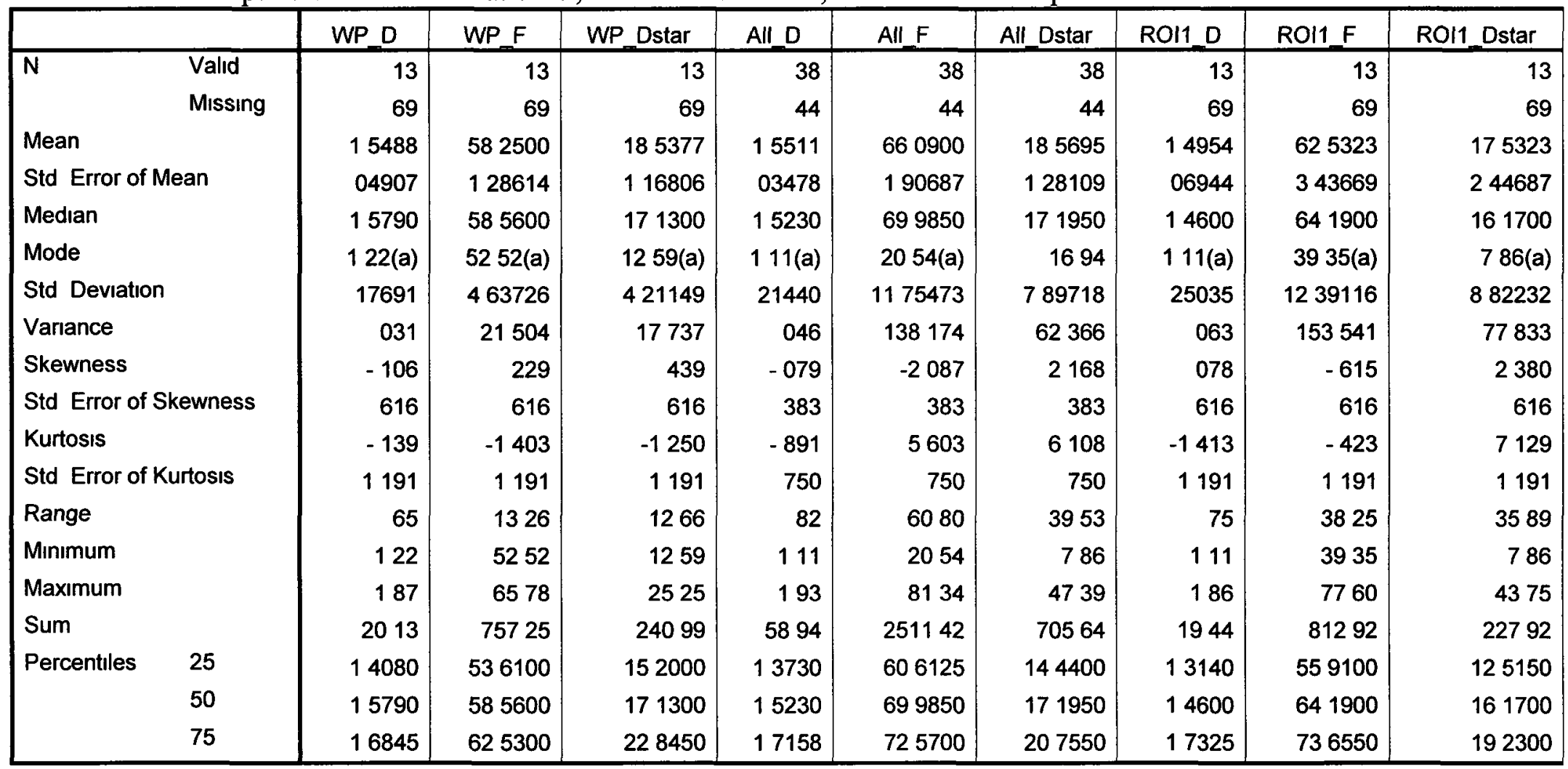

a Multiple modes exist The smallest value is shown 
Table B.7 A normality test was performed on the IVIM parameters for WP-ROI and U-ROI from all subjects investıgated A two tailed asymptotic significance $p$-value is reported

\begin{tabular}{|l|r|r|r|}
\hline & \multicolumn{3}{|c|}{ Shapiro-Wilk } \\
\hline & Statistic & df & Sig \\
\hline WP_D & 954 & 8 & 750 \\
UROI_D & 959 & 8 & 796 \\
ROI1_D & 972 & 8 & 913 \\
WP_Dst & 850 & 8 & 096 \\
UROI_Dst & 811 & 8 & 038 \\
ROI1_Dst & 827 & 8 & 055 \\
WP_f & 943 & 8 & 639 \\
UROI_f & 853 & 8 & 102 \\
ROI1_f & 920 & 8 & 431 \\
\hline
\end{tabular}

This is a lower bound of the true significance

a Lilliefors Significance Correction

Normality assumption is rejected if $p$-value $<005$ 
Table B.8 Descriptive Statistıcs of the parameter distributions, when all placenta subjects are investigated, for WP-ROI, U-ROI and a 100 pixel-ROI=ROIl for $b=50 \mathrm{~s} / \mathrm{mm}^{2}$

Descrıptıve Statıstıcs

\begin{tabular}{|c|c|c|c|c|c|c|c|c|c|}
\hline & \multirow{2}{*}{$\frac{\mathrm{N}}{\text { Statıstıc }}$} & \multirow{2}{*}{$\frac{\text { Mınımum }}{\text { Statıstıc }}$} & \multirow{2}{*}{$\frac{\text { Maxımum }}{\text { Statıstıc }}$} & \multirow{2}{*}{$\frac{\text { Mean }}{\text { Statıstıc }}$} & \multirow{2}{*}{$\frac{\text { Std }}{\text { Statıstic }}$} & \multicolumn{2}{|c|}{ Skewness } & \multicolumn{2}{|c|}{ Kurtosis } \\
\hline & & & & & & Statıstıc & Std Error & Statıstıc & Std Error \\
\hline WP_D & 8 & 155 & 191 & 17662 & 11746 & -780 & 752 & 266 & 1481 \\
\hline UROI_D & 8 & 155 & 183 & 16713 & 09317 & 257 & 752 & 043 & 1481 \\
\hline ROI1_D & 8 & 150 & 186 & 16964 & 11391 & -412 & 752 & 338 & 1481 \\
\hline WP_Dst & 8 & 1854 & 5540 & 324518 & 1072428 & 1349 & 752 & 3412 & 1481 \\
\hline UROI_Dst & 8 & 1846 & 3789 & 243741 & 604954 & 1883 & 752 & 4073 & 1481 \\
\hline ROI1_Dst & 8 & 1753 & 4214 & 258166 & 732536 & 1748 & 752 & 4218 & 1481 \\
\hline WP_f & 8 & 3371 & 4539 & 396958 & 426693 & -264 & 752 & -1257 & 1481 \\
\hline UROI_f & 8 & 3051 & 4348 & 344825 & 428205 & 1501 & 752 & 2371 & 1481 \\
\hline ROl1_f & 8 & 2887 & 4293 & 341548 & 442000 & 1127 & 752 & 1380 & 1481 \\
\hline Valıd N (listwise) & 8 & & & & & & & & \\
\hline
\end{tabular}


APPENDIX C $\widetilde{\theta}$ as a Function of $\theta$ and $N$ Calculated Using $\mu_{\chi}$ and $\sigma_{g}$

The tables in this Appendix contain computed estimates of $\theta_{N}$ as a function of $A$ for the case where the exact values for the NCD mean and $\sigma_{g}$ are used to calculate the RNB correction Since in all cases $\sigma_{g}=1$, it can be seen that $\theta=A, \widetilde{\theta}=\widetilde{A}$ and $\widetilde{\theta}_{N}=\widetilde{A}_{N}$ in the following tables

Table C.1. $\widetilde{\theta}$ as a Function of $\theta$ Calculated Using $\mu_{\chi}$ and $\sigma_{g}$ for $N=1$

$\begin{array}{ccccc}\theta & \mu_{\chi} / \sigma_{g} & \theta_{l} & \tilde{\theta}_{1} & \tilde{\theta} \\ 00000 & 12533 & 00000 & 00014 & 00014 \\ 01000 & 12564 & 01000 & 01007 & 01007 \\ 02000 & 12658 & 02000 & 02005 & 02005 \\ 03000 & 12814 & 03000 & 03004 & 03004 \\ 04000 & 13030 & 04000 & 04003 & 04003 \\ 05000 & 13304 & 05000 & 05001 & 05001 \\ 06000 & 13636 & 06000 & 06010 & 06010 \\ 07000 & 14023 & 07000 & 07009 & 07009 \\ 08000 & 14462 & 08000 & 08008 & 08008 \\ 09000 & 14951 & 09000 & 09007 & 09007 \\ 10000 & 15486 & 10000 & 10006 & 10006 \\ 12000 & 16683 & 12000 & 12004 & 12004 \\ 14000 & 18028 & 14000 & 14002 & 14002 \\ 15000 & 18749 & 15000 & 15002 & 15002 \\ 17500 & 20669 & 17500 & 17501 & 17501 \\ 20000 & 22724 & 20000 & 20000 & 20000 \\ 25000 & 27112 & 25000 & 24999 & 24999 \\ 30000 & 31726 & 30000 & 29998 & 39998 \\ 40000 & 41272 & 40000 & 40012 & 40012\end{array}$

Table C.2 $\tilde{\theta}$ as a Function of $\theta$ Calculated Using $\mu_{\chi}$ and $\sigma_{g}$ for $N=2$

$\begin{array}{ccccc}\theta & \mu_{\chi} / \sigma_{g} & \theta_{2} & \tilde{\theta}_{2} & \tilde{\theta} \\ 00000 & 18800 & 00000 & 00463 & 00327 \\ 05000 & 19381 & 07071 & 06722 & 04753 \\ 10000 & 21058 & 14142 & 13983 & 09887 \\ 15000 & 23652 & 21213 & 21178 & 14965 \\ 20000 & 26945 & 28284 & 28289 & 20003 \\ 25000 & 30731 & 35355 & 35367 & 25012 \\ 30000 & 34851 & 42426 & 42442 & 30011 \\ 40000 & 43689 & 56569 & 56578 & 40001\end{array}$


Table C.3 $\tilde{\theta}$ as a Function of $\theta$ Calculated Using $\mu_{\chi}$ and $\sigma_{g}$ for $N=4$

$\begin{array}{ccccc}\theta & \mu_{\chi} / \sigma_{g} & \theta_{4} & \tilde{\theta}_{4} & \tilde{\theta} \\ 00000 & 27416 & 00000 & 00582 & 00291 \\ 05000 & 27842 & 10000 & 09541 & 04771 \\ 10000 & 29089 & 20000 & 19743 & 09872 \\ 15000 & 31073 & 30000 & 29856 & 14856 \\ 20000 & 33682 & 40000 & 39942 & 19971 \\ 25000 & 36793 & 50000 & 49958 & 24958 \\ 30000 & 40295 & 60000 & 59984 & 29992 \\ 40000 & 48128 & 80000 & 79995 & 39998\end{array}$

Table C.4 $\tilde{\theta}$ as a Function of $\theta$ Calculated Using $\mu_{\chi}$ and $\sigma_{g}$ for $N=8$

$\begin{array}{ccccc}\theta & \mu_{\chi} / \sigma_{g} & \theta_{8} & \widetilde{\theta}_{8} & \tilde{\theta} \\ 00000 & 39380 & 00000 & -00528 & -00187 \\ 05000 & 39687 & 14142 & 13712 & 04848 \\ 10000 & 40594 & 28284 & 28022 & 09907 \\ 15000 & 42067 & 42426 & 42284 & 14858 \\ 20000 & 44054 & 56569 & 56490 & 19972 \\ 25000 & 46493 & 70711 & 70665 & 24984 \\ 30000 & 49320 & 84853 & 84827 & 29991 \\ 40000 & 55909 & 113137 & 113751 & 40614\end{array}$

Table C.5 $\widetilde{\theta}$ as a Function of $\theta$ Calculated Using $\mu_{\chi}$ and $\sigma_{g}$ for $N=16$

$\begin{array}{ccccc}\theta & \mu_{\chi} / \sigma_{g} & \theta_{16} & \tilde{\theta}_{16} & \tilde{\theta} \\ 00000 & 56128 & 00000 & -00423 & -00106 \\ 05000 & 56347 & 20000 & 19654 & 04914 \\ 10000 & 56999 & 40000 & 39780 & 09945 \\ 15000 & 58070 & 60000 & 59872 & 14968 \\ 20000 & 59539 & 80000 & 79914 & 19979 \\ 25000 & 61378 & 10000 & 99951 & 24988 \\ 30000 & 63556 & 12000 & 119988 & 29988\end{array}$


APPENDIX D $\widetilde{\theta}$ as a Function of $\theta$ and $N$ Calculated Using $\left\langle M_{N}\right\rangle_{\chi^{9}}$ and $\sigma_{g}$

The tables in this Appendix contain computed estımates of $\theta_{N}$ as a function of $A$ for the case where $\mu_{x}$ was approximated by a "nearest neighbour average" of NCD distributed pixel intensities in the calculation of the RNB correction $\mu_{x} / \sigma_{g}$ represents the theoretical value Since in all cases $\sigma_{g}=1$, it can be seen that $\theta=A, \widetilde{\theta}=\widetilde{A}$ and $\widetilde{\theta}_{N}=\widetilde{A}_{N}$ in the following tables The number of near neighbours used was 9 for all of these sımulations

Table D.1. $\tilde{\theta}$ as a Function of $\theta$ Calculated Using $\left\langle M_{N}\right\rangle_{\chi 9}$ and $\sigma_{g}$ for $N=1$

$\begin{array}{ccccc}\theta & \mu_{\chi} / \sigma_{g} & \theta_{1} & \tilde{\theta}_{1} & \tilde{\theta} \\ 00000 & 12533 & 00000 & 0256 & 0256 \\ 01000 & 12564 & 01000 & 0265 & 0265 \\ 02000 & 12658 & 02000 & 0285 & 0285 \\ 03000 & 12814 & 03000 & 0320 & 0320 \\ 04000 & 13030 & 04000 & 0369 & 0369 \\ 05000 & 13304 & 05000 & 0433 & 0433 \\ 06000 & 13636 & 06000 & 0507 & 0507 \\ 07000 & 14023 & 07000 & 0594 & 0594 \\ 08000 & 14462 & 08000 & 0691 & 0691 \\ 09000 & 14951 & 09000 & 0794 & 0794 \\ 10000 & 15486 & 10000 & 0902 & 0902 \\ 12000 & 16683 & 12000 & 1127 & 1127 \\ 14000 & 18028 & 14000 & 1349 & 1349 \\ 15000 & 18749 & 15000 & 1459 & 1459 \\ 17500 & 20669 & 17500 & 1725 & 1725 \\ 20000 & 22724 & 20000 & 1985 & 1985 \\ 25000 & 27112 & 25000 & 2493 & 2493 \\ 30000 & 31726 & 30000 & 2996 & 2996 \\ 40000 & 41272 & 40000 & 4005 & 4005\end{array}$

Table D.2 $\tilde{\theta}$ as a Function of $\theta$ Calculated Using $\left\langle M_{N}\right\rangle_{x 9}$ and $\sigma_{g}$ for $N=2$

$\begin{array}{ccccc}\theta & \mu_{\chi} / \sigma_{g} & \theta_{2} & \widetilde{\theta}_{2} & \tilde{\theta} \\ 00000 & 18800 & 00000 & 03198 & 02261 \\ 05000 & 19951 & 07071 & 06050 & 04278 \\ 10000 & 23151 & 14142 & 13388 & 09467 \\ 15000 & 27826 & 21213 & 20992 & 14844 \\ 20000 & 33407 & 28284 & 28200 & 19940 \\ 25000 & 39509 & 35355 & 35307 & 24966 \\ 30000 & 45911 & 42426 & 42403 & 29983\end{array}$




$\begin{array}{lllll}35000 & 52496 & 49497 & 49487 & 34993 \\ 40000 & 59199 & 56569 & 56555 & 34993\end{array}$

Table D.3 $\tilde{\theta}$ as a Function of $\theta$ Calculated Using $\left\langle M_{N}\right\rangle_{\chi 9}$ and $\sigma_{g}$ for $N=4$

$\begin{array}{ccccc}\theta & \mu_{\chi} / \sigma_{g} & \theta_{4} & \tilde{\theta}_{4} & \tilde{\theta} \\ 00000 & 27416 & 00000 & 03934 & 01967 \\ 05000 & 29089 & 10000 & 08666 & 04333 \\ 10000 & 33682 & 20000 & 19514 & 09757 \\ 15000 & 40295 & 30000 & 29868 & 14934 \\ 20000 & 48128 & 40000 & 39944 & 19972 \\ 25000 & 56671 & 50000 & 49970 & 24985 \\ 30000 & 65639 & 60000 & 59984 & 29992 \\ 35000 & 74876 & 70000 & 69985 & 34993 \\ 40000 & 84292 & 80000 & 79990 & 39995\end{array}$

Table D.4 $\tilde{\theta}$ as a Function of $\theta$ Calculated Using $\left\langle M_{N}\right\rangle_{\chi^{9}}$ and $\sigma_{g}$ for $N=8$

$\begin{array}{ccccc}\theta & \mu_{\chi} / \sigma_{g} & \theta_{8} & \tilde{\theta}_{8} & \tilde{\theta} \\ 00000 & 39380 & 00000 & 04760 & 01683 \\ 05000 & 41776 & 14142 & 12579 & 04447 \\ 10000 & 48310 & 28284 & 27974 & 09890 \\ 15000 & 57651 & 42426 & 42328 & 14965 \\ 20000 & 68677 & 56569 & 56520 & 19983 \\ 25000 & 80696 & 70711 & 70681 & 24990 \\ 30000 & 93321 & 84853 & 84835 & 29994 \\ 35000 & 10633 & 98995 & & \\ 40000 & 12137 & 11314 & 11294 & 39931\end{array}$

Table D.5 $\tilde{\theta}$ as a Function of $\theta$ Calculated Using $\left\langle M_{N}\right\rangle_{\chi 9}$ and $\sigma_{g}$ for $N=16$

$\begin{array}{ccccc}\theta & \mu_{\chi} / \sigma_{g} & \theta_{16} & \tilde{\theta}_{16} & \tilde{\theta} \\ 00000 & 56128 & 00000 & 05813 & 01453 \\ 05000 & 59539 & 20000 & 18458 & 04615 \\ 10000 & 68801 & 40000 & 39794 & 09949 \\ 15000 & 81997 & 60000 & 59923 & 14981 \\ 20000 & 97553 & 80000 & 79980 & 19995 \\ 25000 & 11451 & 10000 & 99972 & 24993 \\ 30000 & 13232 & 12000 & 11986 & 29965 \\ 35000 & 15070 & 14000 & 13985 & 34963 \\ 40000 & 16942 & 16000 & 15984 & 39961\end{array}$


APPENDIX E Simulated PDFs Before and After the RNBC Calculated Using $\left\langle M_{N}\right\rangle_{x 9}$ and $\sigma_{g}$ for Different $N$ Element Colls

The figures in this Appendix contain simulated PDFs before (solid) and after (dashed) the RNB correction in the 9-nearest neighbour approximation for $N=1$ (Fig E 1), $N=2$ (Fig E 2), $N=4$ (Fig E 3), $N=8$ (Fig E 4) and $N=16$ (Fig E 5) elements with $\theta=05$ The vertical lines show the corresponding PDF means for the corrected (dashed) and uncorrected (solid) PDFs for the respective number of colls

Figure E.1 Simulated PDFs Before and After the RNBC Calculated Using $\left\langle M_{N}\right\rangle_{x^{9}}$ and $\sigma_{g}$ for $N=1$

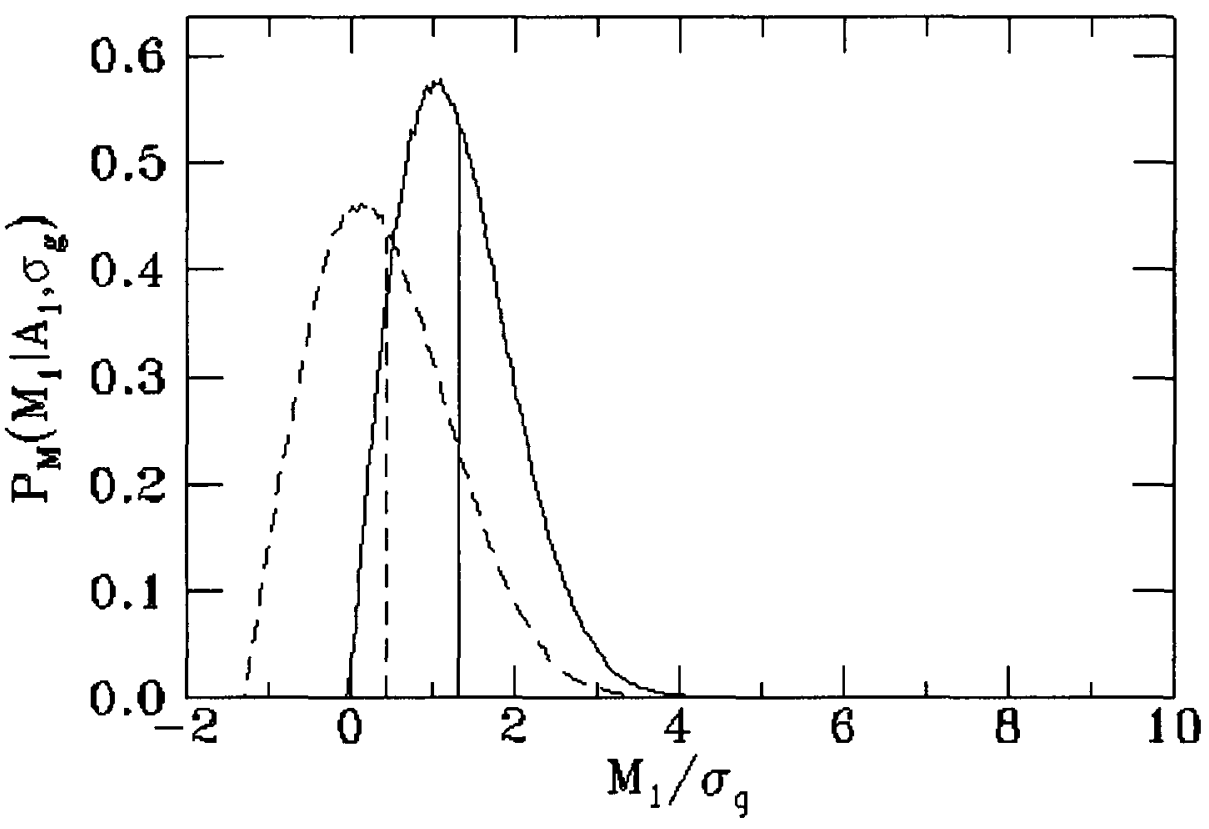


Figure E.2 Simulated PDFs Before and After the RNBC Calculated Using $\left\langle M_{N}\right\rangle_{\chi^{9}}$ and $\sigma_{g}$ for $N=2$

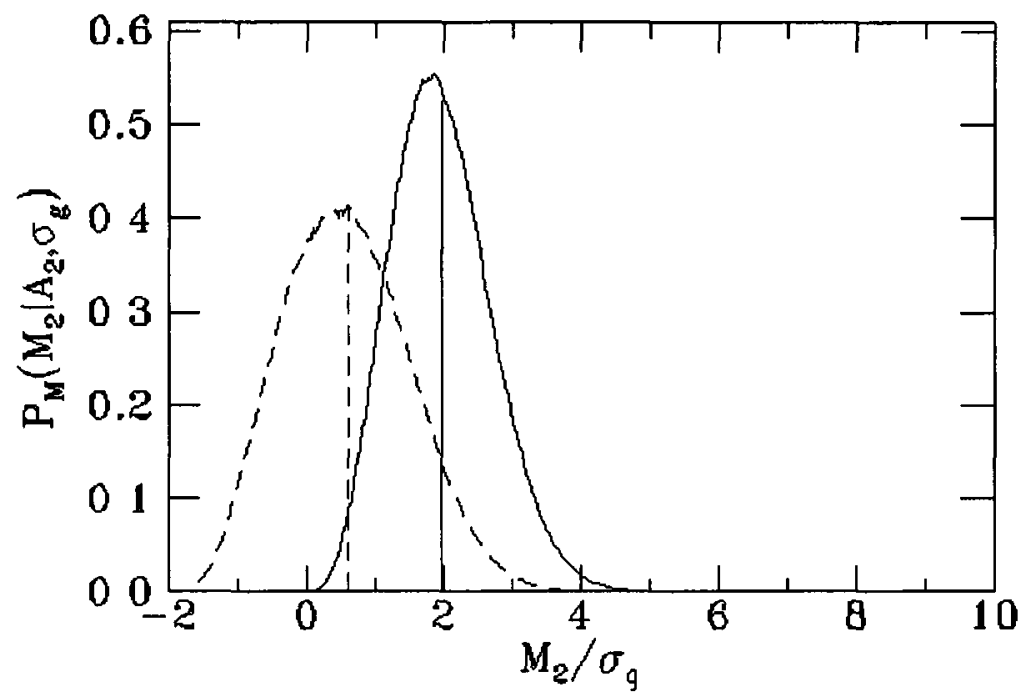

Figure E.3 Simulated PDFs Before and After the RNBC Calculated Using $\left\langle M_{N}\right\rangle_{\chi^{9}}$ and $\sigma_{g}$ for $N=4$

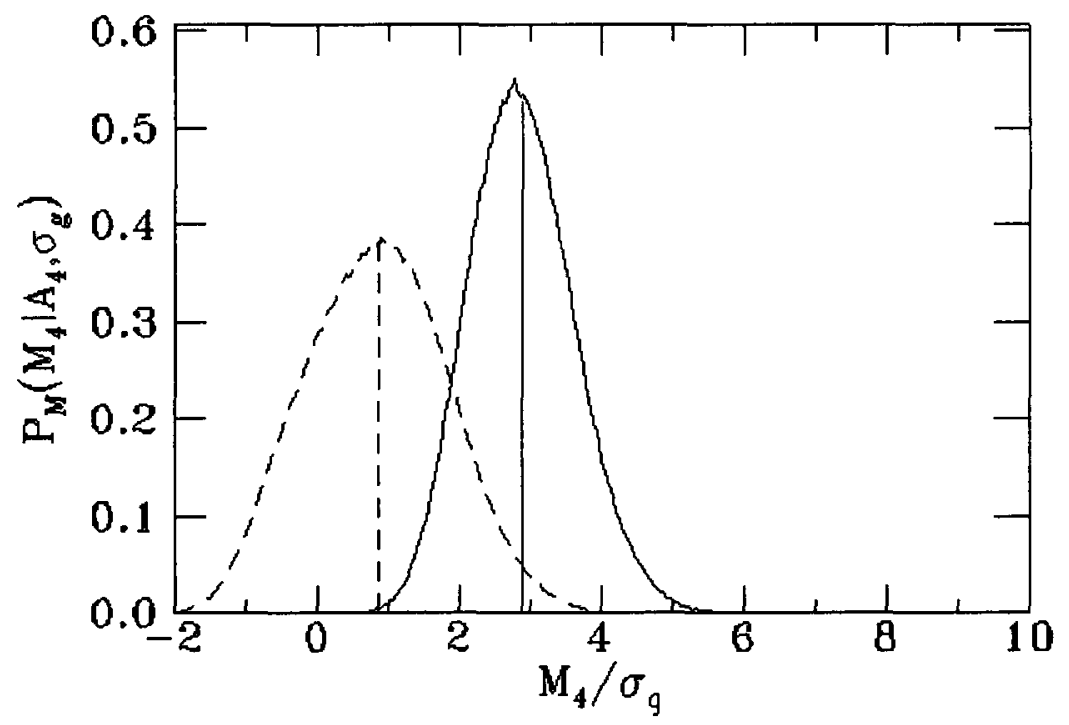


Figure E.4 Simulated PDFs Before and After the RNBC Calculated Using $\left\langle M_{N}\right\rangle_{\chi^{9}}$ and $\sigma_{g}$ for $N=8$

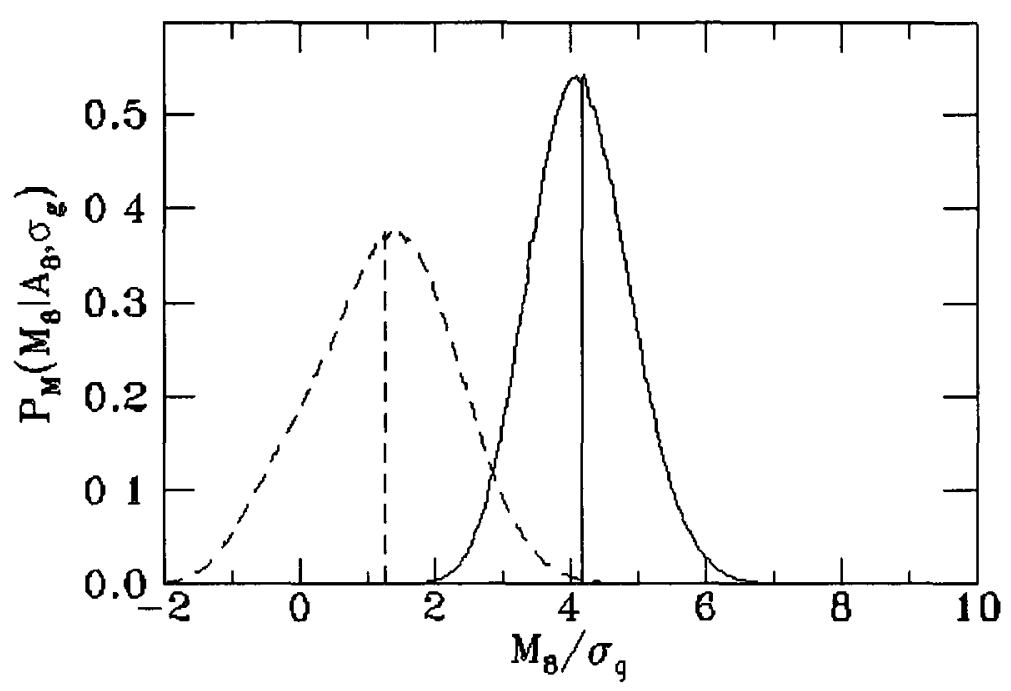

Figure E.5 Simulated PDFs Before and After the RNBC Calculated Using $\left\langle M_{N}\right\rangle_{\chi^{9}}$ and $\sigma_{g}$ for $N=16$

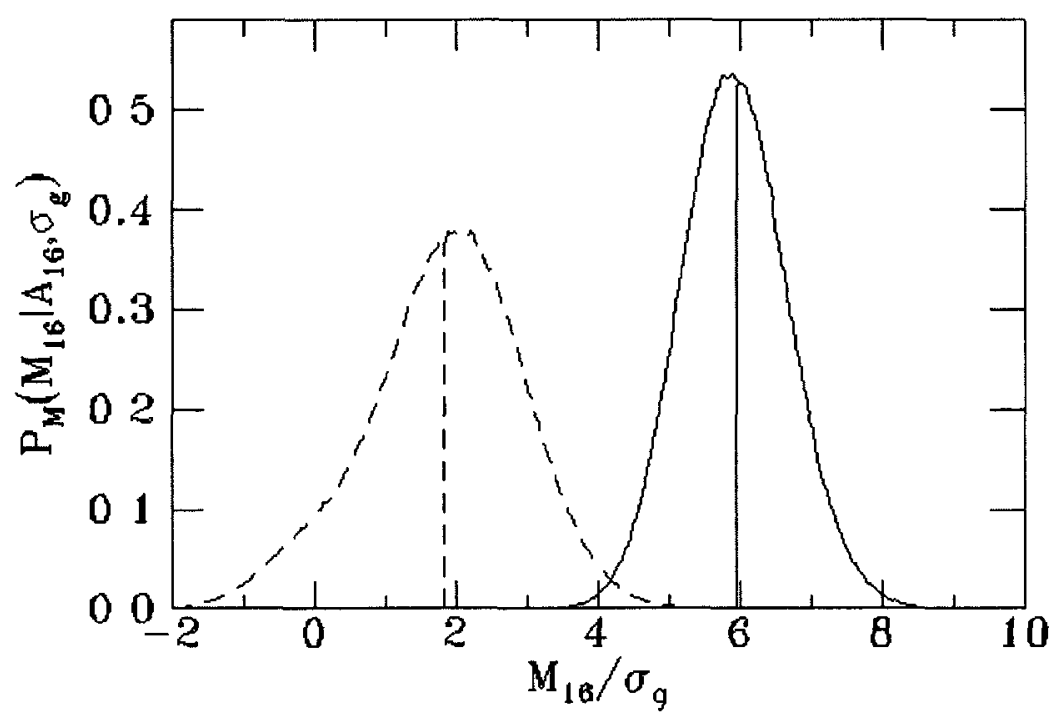




\section{References}

[1] Mansfield P and Grannell P K NMR 'diffraction' in solıds? J Phys C Solıd State Phys 1973,6 L442

[2] Lauterbur P Image formation by induced local interactions Examples employing NMR Nature 1973, 242 190-191

[3] Kumar A, Weltı D, Ernst R NMR Fourıer zeugmatography J Magn Reson 1975, $1869-83$

[4] E O Stejskal and J E Tanner, Spın diffusion measurements Spin echoes in the presence of a tıme-dependent field gradient J Chem Phys, 42(1) 288-292,1965

[5] Le Bihan D, Breton E, Syrota A Imagerie de diffusion par resonance magnetique nucleaire C R Acad Sc1 [Ill] 1985, 301 1109-1112

[6] A Einstein, Investigations on the Theory of the Brownian Movement, Courier Dover Publications, (1956)

[7] Le Bihan D, Intravoxel Incoherent Motion Perfusion MR Imaging A Wake-Up Call, Radiology 2008, 249 748-752

[8] Le Bihan D, Moonen CT, Van Zijl PC, Pekar J, Des Pres D Measurıng random microscopic motion of water in tissues with MR imaging a cat brain study J Comput Assist Tomogr 1991,15 19-25

[9] Lucianı A et al Liver cirrhosıs intravoxel incoherent motion MR ımaging- pilot study Radiology 2008,249(3) 891-899 
[10] Namımoto T, Yamashita Y, Sum1 S, Tang Y, Takahashı M Focal liver masses characterization with diffusion-weighted echo-planar MR imaging Radiology $1997,204739-744$

[11] Ichıkawa T, Haradome H, Hachıya J, Nitatorı T, Arakı T Diffusıon weighted MR Imaging with a single-shot echoplanar sequence detection and characterization of focal hepatıc lesions Am J Roentgenol 1998,170 397-402

[12] Yamada I, Aung W, Hımeno Y, Nakagawa T, Shibuya H Diffusion coefficients in abdominal organs and hepatic lesions evaluation with intravoxel incoherent motion echo-planar MR ımaging Radılogy 1999,210 617-623

[13] Kım T, Murakamı T, Takahashı S, Horı M, Tsuda K, Nakamura H Dıffusionweighted single-shot echoplanar MR imaging for liver disease Am J Roentgenol $1999,173393-398$

[14] Taoulı B, Vilgrain V, Dumont E, Daire JL, Fan B, Menu Y Evaluation of liver diffusion isotropy and characterization of focal hepatic lesions with two single-shot echoplanar MR imaging sequences prospective study in 66 patients Radiology 2003,226 7178

[15] Hussain SM, De Becker J, Hop WC, Dwarkasing S, Wielopolskı PA Can a singleshot black-blood T2-weighted spin-echo echo-planar imaging sequence with sensitivity encoding replace the respiratory triggered turbo spin-echo sequence for the liver? An optımization and feasibility study J Magn Reson Imaging 2005,21 219-229

[16] Coenegrachts $\mathrm{K}$ et al Improved focal liver lesion detection comparison of singleshot diffusion-weighted echoplanar and single-shot $\mathrm{T} 2$ weighted turbo spin echo techniques Br J Radıol 2007,80 524-531 
[17] Bruegel M, Gaa J, Waldt S, et al Diagnosis of hepatic metastasis comparison of respiration-triggered diffusion-weighted echo-planar MRI and five t2-weighted turbo spin-echo sequences Am J Roentgenol 2008,191 1421-1429

[18] Bruegel M, Holzapfel K, Gaa J, et al Characterization of focal liver lesions by ADC measurements using a respiratory triggered diffusion-weighted single-shot echo-planar MR imaging technique Eur Radiol 2008,18 477-485

[19] Parkh $T$ et al Focal liver lesion detection and characterization with diffusionweighted MR imaging comparison with standard breath-hold T2 weighted imaging Radiology 2008,246 812-822

[20] Muller MF, Prasad P, Siewert B, Nissenbaum MA, Raptopoulos V, Edelman RR Abdominal diffusion mapping with use of a wholebody echo-planar system Radiology $1994,190475-478$

[21] Motek1 $\mathrm{T}$, Ishizaka $\mathrm{H}$, Horikosh1 $\mathrm{H}$, Matsumoto $\mathrm{M}$ Differentiation between hemangiomas and hepatocellular carcinomas with the apparent diffusion coefficient calculated from turboFLASH MR images J Magn Reson Imaging 1995,5 187-191

[22] Okada Y, Ohtomo K, Kıryu S, Sasakı Y Breath-hold T2-weıghted MRI of hepatıc tumors value of echo planar imaging with diffusion sensitizing gradient J Comput Assist Tomogr 1998,22 364-371

[23] Ichıkawa T, Haradome H, Hachıya J, Nitatorı T, Arakı T Diffusıon weıghted MR ımaging with single-shot echo-planar imaging in the upper abdomen prelıminary clinical experience in 61 patients Abdom Imaging 1999,24 456-461

[24] Ichıkawa $T$, Arakı $T$ Fast magnetıc resonance ımaging of liver Eur J Radıol $1999,29186-210$ 
[25] Motekı T, Sekıne T Echo planar MR ımaging of the liver comparıson of images with and without motion probing gradients J Magn Reson Imaging 2004,19 82-90

[26] Koinuma M, Ohashı I, Hanafusa K, Shıbuya H Apparent diffusion coefficient measurements with diffusion-weighted magnetic resonance imaging for evaluation of hepatıc fibrosis J Magn Reson Imaging 2005,22 80-85

[27] Nasu $\mathrm{K}$ et al Hepatic metastases diffusion weighted sensitıvity-encodıng versus SPIO-enhanced MR imaging Radiology 2006,239 122-130

[28] Koh DM et al Detection of colorectal hepatic metastases using MnDPDP MR ımaging and diffusion-weighted ımaging (DWI) alone and in combination Eur Radiol $2008,18903-910$

[29] Gıromettı R, Furlan A, Bazzocchı M, et al Diffusion-weighted MRI in evaluatıng liver fibrosis a feasibility study in cirrhotic patients Radiol Med (Torino) 2007,112 394408

[30] Taoulı B et al Diffusion-weighted MRI for quantification of liver fibrosis prelımınary experıence Am J Roentgenol 2007,189 799-806

[31] Taoulı B, Choulı M, Martın AJ, Qayyum A, Coakley FV, Vilgrain V Chronıc hepatitis role of diffusion-weighted imaging and diffusion tensor imaging for the diagnosis of liver fibrosıs and inflammation J Magn Reson Imaging 2008,28 89-95

[32] Koinuma M, Ohashı I, Hanafusa K, Shıbuya H Apparent diffusion coefficient measurements with diffusion-weighted magnetıc resonance imaging for evaluation of hepatıc fibrosıs J Magn Reson Imaging 2005,22 80-85

[33] Lewin $M$ et al Diffusion-weighted magnetic resonance imaging for the assessment of fibrosis in chronic hepatitıs C Hepatology 2007,46 658-665 
[34] Kamel IR et al Role of diffusion-weighted imaging in estımatıng tumor necrosis after chemoembolization of hepatocellular carcınoma Am J Roentgenol 2003,181 708710

[35] Kamel IR et al The role of functional MR imaging in the assessment of tumor response after chemoembolization in patients with hepatocellular carcinoma $J$ Vasc Interv Radiol 2006,17 505-512

[36] Koh DM et al Predicting response of colorectal hepatic metastasis value of pretreatment apparent diffusion coefficients Am J Roentgenol 2007,188 1001-1008 [37] Cuı Y, Zhang XP, Sun YS, Tang L, Shen L Apparent diffusion coefficient potential imaging biomarker for prediction and early detection of response to chemotherapy in hepatıc metastases Radıology 2008,248 894-900

[38] Kamel IR, Reyes DK, Liapı E, Bluemke DA, Geschwind JF Functional MR imaging assessment of tumor response after ${ }^{90} \mathrm{Y}$ microsphere treatment in patients with unresectable hepatocellular carcinoma J Vasc Interv Radıl 2007,18 49-56

[39] Chen CY et al Early response of hepatocellular carcinoma to transcatheter arterial chemoembolization choline levels and MR diffusion constants-initial experience Radıology 2006,239 448-456

[40] Liap1 E et al Functional MRI evaluation of tumor response in patients with neuroendocrine hepatic metastasis treated with transcatheter arterial chemoembolization Am J Roentgenol 2008,190 67-73

[41] Kamel IR, Liapı E, Reyes DK, Zahurak M, Bluemke DA, Geschwind JF Unresectable hepatocellular carcinoma serial early vascular and cellular changes after 
transarterial chemoembolization as detected with MR imaging Radiology 2009,250 466 473

[42] Koh DM et al Colorectal hepatıc metastases quantıtatıve measurements using single-shot echo-planar diffusion-weighted MR ımaging Eur Radıol 2006, 161898 1905

[43] Koh DM, Collins DJ Diffusion-Weighted MRI in the Body Applications and Challenges in Oncology, Am J Roentgenol 2007, 188 1622-1635

[44] Bammer R, Keelıng SL, Augustın M, et al Improved diffusion weighted sıngle-shot echo-planar imaging (EPI) in stroke using sensitıvity encoding (SENSE) Magn Reson Med 2001,46 548-554

[45] Taoulı B, Martın AJ, Qayyum A, et al Parallel ımaging and dıffusion tensor ımaging for diffusion-weighted MRI of the liver prelıminary experience in healthy volunteers AJR Am J Roentgenol 2004,183 677-680

[46] Murtz P, Flacke S, Traber F, van den Brınk JS, Gieseke J, Schıld HH Abdomen diffusion-weighted MR imaging with pulse-triggered single-shot sequences Radiology $2002,224258-264$

[47] Nasu K, Kurokı Y, Sekıguchı R, Nawano S The effect of sımultaneous use of respiratory triggering in diffusion-weighted imaging of the liver Magn Reson Med Sc1 $2006,5129-136$

[48] Gourtsoyıannı S, Papanıkolaou N, Yarmenıtıs S, Marıs T, Karantanas A, Gourtsoyiannıs N Respiratory gated diffusion-weighted imaging of the liver value of apparent diffusion coefficient measurements in the differentiation between most 
commonly encountered benign and malignant focal liver lesions Eur Radiol $2008,18486-492$

[49] Kwee TC, Takahara T, Koh DM, Nievelstein RA, Luyten PR Comparison and reproducibility of $\mathrm{ADC}$ measurements in breath hold, respiratory triggered, and freebreathing diffusion-weighted MR imaging of the liver $J$ Magn Reson Imaging $2008,281141-1148$

[50] Asbach $\mathrm{P}$ et al Magnetic resonance cholangıopancreatography using a freebreathing T2-weighted turbo spin-echo sequence with navigator-triggered prospective acquisition correction Magn Reson Imaging 2005,23 939-945

[51] Wang Y, Rossman PJ, Grımm RC, Riederer SJ, Ehman RL Navigator-echo-based real-tıme respıratory gatıng and trıggerıng for reduction of respiration effects in threedımensıonal coronary MR angıography Radıology 1996,198 55-60

[52] Spritzer CE, Keogan MT, DeLong DM, Dahlke J, MacFall JR Optımızıng fast spın echo acquisitions for hepatic ımaging in normal subjects J Magn Reson Imaging $1996,6128-135$

[53] Low RN, Alzate GD, Shimakawa A Motion suppression in MR imaging of the liver comparison of respiratory-triggered and nontriggered fast spin-echo sequences Am $J$ Roentgenol 1997,168 225-231

[54] Klessen $\mathrm{C}$, et al Magnetıc resonance ımaging of the upper abdomen using a freebreathıng T2-weighted turbo spin echo sequence with navigator triggered prospective acquisition correction J Magn Reson Imaging 2005,21 576-582

[55] Cho1 JY, Lee JM, Lee JY, et al Navigator-trıggered isotropic three dımensıonal magnetic resonance cholangiopancreatography in the diagnosis of malignant biliary 
obstructions comparıson with direct cholangıography J Magn Reson Imaging $2008,2794-101$

[56] Moore RJ, Issa $\mathrm{B}$, Tokarczuk $\mathrm{P}$, et al In vivo intravoxel incoherent motion measurements in the human placenta using echo-planar ımaging at $05 \mathrm{~T}$ Magn Reson Med 2000,43 295-302

[57] Moore RJ, Strachan BK, Tyler DJ, et al In utero perfusing fraction maps in normal and growth restricted pregnancy measured using IVIM echo-planar MRI Placenta $2000,21726-732$

[58] A M Al-Radardeh1et al, Proc Intl Soc Mag Reson Med 2009, p 17

[59] Henkelman RM Does IVIM measure classical perfusion? Magn Reson Med $1990,16470-475$

[60] S Truica, G Cron, I Cameron, Improved DiffusionWeighted MR Imaging of Liver in Normal Subjects, Med Phys 36, 4322 (2009), do1 10 1118/1 3244191

[61] Truica L S , Cameron I, Gruslin A, Avruch L , Assessment of Placental Blood Flow Usıng a Navigator Echo Respıratory Gated Parallel Imaging Technıque at 15 T, ISMRM $2010, \mathrm{p} 4564$

[62] Slichter C P , Principles of Magnetıc Resonance (Springer-Verlag, New York 1990)

[63] Cowan B, Nuclear Magnetic Resonance and Relaxation (Cambridge University Press, Cambridge 1997)

[64] Callaghan P T, Principles of Nuclear Magnetic Resonance Microscopy (Clarendon Press, Oxford 1991)

[65] Carr H Y and Purcell E M, The effect of diffusion on free precession in nuclear magnetic resonance, Phys Rev 1954, 94 630-8 
[66] Meıboom S and Gill D, Modified spin-echo method for measuring nuclear relaxation times, Rev Scı Instr 1958, 29 688-91

[67] Crank J, The mathematıcs of diffusion Oxford, England Oxford Unıversity Press, 1975

[68] Jost W , Diffusion in solids, liquids and gases New York Academıc Press, 1960

[69] Pathria R K , Statıstical Mechanics Pergamon Press, 1972

[70] Hahn E L Spın echoes, Phys Rev, 1950, 80(4) 580-594

[71] Carr $\mathrm{H} \mathrm{Y}$ and Purcell E M, Effects of diffusion on free precession in Nuclear Magnetıc Resonance Experıments Phys Rev, 1954, 94(3) 630

[72] Tanner J E , The use of stımulated echo in NMR diffusion studies $J$ Chem Phys, $1965,190,522523-2526$

[73] Torrey H C , Bloch equations with diffusion terms Phys Rev ,1956, 104563

[74] D Le Bihan, E Breton, D Lallemand, P Grenier, E Cabanıs, and M Laval-Jeantet MR imaging of intravoxel incoherent motions application to diffusion and perfusion in neurologic disorders Radıology, 1986, 161(2) 401-7

[75] Y Cohen and Y Assaf, High b-value q-space analyzed diffusion-weighted MRS and MRI in neuronal tissues - a technical review, NMR Biomed, 2002, 15(7-8) 516-542

[76] Le Bıhan D, Breton E, Lallemand D, Aubın ML, Vıgnaud J, Laval Jeantet M Separation of diffusion and perfusion in intravoxel incoherent motion (IVIM) MR Imaging Radıology 1988,168 497-505

[77] Ehman RL, Felmlee JP, Adaptıve technıque for high-definition MR imaging of moving structures, Radılogy 1989 Oct, 173(1) 255-63 
[78] Thesen S, Heid O, Mueller E, Schad LR, Prospectıve acquisition correction for head motion with image-based trackıng for real-tıme fMRI, Magn Reson Med 2000, $44(3) 457-65$

[79] Gray H, Anatomy of the Human Body, $15^{\text {th }}$ Edition, Chancellor Press, Finland, 1994

[80] Mills R, Self-Diffusion in Normal and Heavy Water in the Range 1-45 J Phys Chem , 1973,77 685-688

[81] Press W H , Teukolsky S A, Vetterling W T, Flannery B P, Numerical Recıpes, Second Edition, Cambridge University Press, 1992

[82] Reese T G, Heid O, Weissko R M, Wedeen V J, Reduction of eddy current induced distortion in diffusion MRI using a twice-refocused spin echo Magn Reson Med, 2003, 49(1) 177-182

[83] Mayhew et al, Placental Morphogenesis and the star volumes of villous trees and intervillous pores, Placenta 1994, 15 209-217

[84] Bergmann A, Zygmunt M, Clapp JF, Running throughout pregnancy effect on placental villous vascular volume and cell proliferation, Placenta, September 2004, 25 8, 694-698

[85] Le Bihan D editor, Diffusion and Perfusion Magnetıc Resonance Imaging Applications to Functional MRI, Raven Press, New York, 1995

[86] C G Koay, P J Basser, J Magn Reson 179 (2006), 317-322

[87] A Cardenas-Blanco et al, Accurate noise bias correction applied to individual pixels, p 3445, ISMRM Berlın (2007), 952-962

[88] P Tofts, Quantttattve MRI of the Brain, Wiley (2003) 
[89] D K Jones, P J Basser, Squashıng peanuts and smashıng pumpkıns" how noise distorts diffusion- weighted MR data Magn Reson Med 52 (2004), 979-993

[90] A Cárdenas-Blanco, C Tejos, P Irarrazaval, I G Cameron, Noıse in Magnitude Magnetıc Resonance Images, Concepts in Magn Reson (2008), 32A(6), 409-416

[91] C D Constantınıdes et al, Signal to Noise Measurements in Magnitude Images from NMR Phased ArraysMagn Reson Med 38 (1997), 851-857

[92] P B Roemer et al, Magn Reson Med 16 (1990), 192-225

[93] M H Ross, Histology A Text and Atlas With Correlated Cell and Molecular Bıology, Lippincott Williams \& W1lkins, published 2010-10-15

[94] D W Fawcett, $A$ textbook of Histology, $12^{\text {th }}$ Edition, Chapman \&Hall Press, New York, 1994

[95] J Patel, E Sigmund, H Rusinek, M Oe1, J S Babb and B Taoul, Diagnosis of cirrhosis with intravoxel incoherent motion diffusion MRI and dynamic contrastenhanced MRI alone and in combination Prelıminary experience, J Magn Reson Imaging 31(3) 589-600 (2010)

[96] S D Flamm, Redıstribution of Regional and Organ Blood Volume and Effect on Cardiac Function in Relation to Upright Exercise Intensity in Healthy Human Subjects, Circulation 1990,81,1550-1559

[97] Lautt WW, Greenway CV, Conceptual review of the hepatıc vascular bed, Hepatology 1987,7 952-963

[98] M A Bernsteın, K F Kıng, X J Zhou, Handbook of MRI Pulse Sequences, Elsevier Academıc Press, USA, 2004 